Cochrane Database of Systematic Reviews

\title{
Smoking reduction interventions for smoking cessation (Review)
}

Lindson N, Klemperer E, Hong B, Ordóñez-Mena JM, Aveyard P

Lindson N, Klemperer E, Hong B, Ordóñez-Mena JM, Aveyard P.

Smoking reduction interventions for smoking cessation.

Cochrane Database of Systematic Reviews 2019, Issue 9. Art. No.: CD013183.

DOI: 10.1002/14651858.CD013183.pub2.

www.cochranelibrary.com 
TABLE OF CONTENTS

HEADER

ABSTRACT

PLAIN LANGUAGE SUMMARY

SUMMARY OF FINDINGS

BACKGROUND

OBJECTIVES

METHODS

RESULTS

Figure 1.

Figure 2.

DISCUSSION

Figure 3.

Figure 4.

AUTHORS' CONCLUSIONS

ACKNOWLEDGEMENTS

REFERENCES

CHARACTERISTICS OF STUDIES

DATA AND ANALYSES

Analysis 1.1. Comparison 1 Reduction to quit versus no treatment, Outcome 1 Abstinence.

Analysis 1.2. Comparison 1 Reduction to quit versus no treatment, Outcome 2 Abstinence: subgrouped by pre-quit pharma in reduction arm.

Analysis 1.3. Comparison 1 Reduction to quit versus no treatment, Outcome 3 Quit attempts.

Analysis 1.4. Comparison 1 Reduction to quit versus no treatment, Outcome 4 Pre-quit reduction in cpd of at least $50 \%$. .......

Analysis 1.5. Comparison 1 Reduction to quit versus no treatment, Outcome 5 Pre-quit SAEs.

Analysis 2.1. Comparison 2 Reduction to quit versus abrupt quitting, Outcome 1 Abstinence.

Analysis 2.2. Comparison 2 Reduction to quit versus abrupt quitting, Outcome 2 Abstinence: sensitivity analysis removing studies with lower-intensity abrupt arms.

Analysis 2.3. Comparison 2 Reduction to quit versus abrupt quitting, Outcome 3 Abstinence: subgrouped by pre-quit pharma in reduction arm.

Analysis 2.4. Comparison 2 Reduction to quit versus abrupt quitting, Outcome 4 Abstinence: subgrouped by set quit date. ....

Analysis 2.5. Comparison 2 Reduction to quit versus abrupt quitting, Outcome 5 Abstinence: subgrouped by cpd vs sfp reduction.

Analysis 2.6. Comparison 2 Reduction to quit versus abrupt quitting, Outcome 6 Abstinence: subgrouped by structured vs unstructured reduction advised.

Analysis 2.7. Comparison 2 Reduction to quit versus abrupt quitting, Outcome 7 Abstinence: subgrouped by length of the reduction period.

Analysis 2.8. Comparison 2 Reduction to quit versus abrupt quitting, Outcome 8 Abstinence: subgrouped by reduction goal. .. Analysis 2.9. Comparison 2 Reduction to quit versus abrupt quitting, Outcome 9 Quit attempts.

Analysis 2.10. Comparison 2 Reduction to quit versus abrupt quitting, Outcome 10 Pre-quit reduction of at least $50 \%$. ...........

Analysis 2.11. Comparison 2 Reduction to quit versus abrupt quitting, Outcome 11 Pre-quit reduction in cpd. ........................

Analysis 2.12. Comparison 2 Reduction to quit versus abrupt quitting, Outcome 12 Pre-quit reduction in carbon monoxide. ...

Analysis 2.13. Comparison 2 Reduction to quit versus abrupt quitting, Outcome 13 Pre-quit reduction in cotinine. .................

Analysis 2.14. Comparison 2 Reduction to quit versus abrupt quitting, Outcome 14 Pre-quit SAEs.

Analysis 2.15. Comparison 2 Reduction to quit versus abrupt quitting, Outcome 15 Pre-quit tobacco withdrawal \& additional AE information.

Analysis 2.16. Comparison 2 Reduction to quit versus abrupt quitting, Outcome 16 Moderation of the reduction vs abrupt quitting effect.

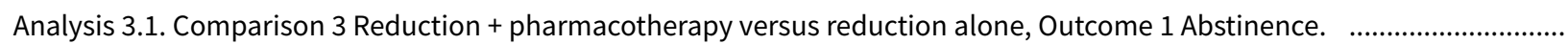

Analysis 3.2. Comparison 3 Reduction + pharmacotherapy versus reduction alone, Outcome 2 Quit attempts. ........................

Analysis 3.3. Comparison 3 Reduction + pharmacotherapy versus reduction alone, Outcome 3 Pre-quit cpd reduction of $\geq 50 \%$ or $75 \%$.

Analysis 3.4. Comparison 3 Reduction + pharmacotherapy versus reduction alone, Outcome 4 Pre-quit reduction in cpd. ....... 
Analysis 3.5. Comparison 3 Reduction + pharmacotherapy versus reduction alone, Outcome 5 Pre-quit reduction in carbon monoxide.

Analysis 3.6. Comparison 3 Reduction + pharmacotherapy versus reduction alone, Outcome 6 Pre-quit AEs.

Analysis 3.7. Comparison 3 Reduction + pharmacotherapy versus reduction alone, Outcome 7 Pre-quit SAEs.

Analysis 3.8. Comparison 3 Reduction + pharmacotherapy versus reduction alone, Outcome 8 Pre-quit tobacco withdrawal \& additional $A E$ information.

Analysis 4.1. Comparison 4 Modality of reduction support, Outcome 1 Abstinence.

Analysis 4.2. Comparison 4 Modality of reduction support, Outcome 2 Pre-quit reduction in cpd.

Analysis 5.1. Comparison 5 Length of reduction, Outcome 1 Abstinence.

Analysis 5.2. Comparison 5 Length of reduction, Outcome 2 Pre-quit cpd reduction $\geq 50 \%$.

Analysis 5.3. Comparison 5 Length of reduction, Outcome 3 Pre-quit tobacco withdrawal.

Analysis 6.1. Comparison 6 More structured vs less structured reduction, Outcome 1 Abstinence.

Analysis 6.2. Comparison 6 More structured vs less structured reduction, Outcome 2 Quit attempts.

Analysis 6.3. Comparison 6 More structured vs less structured reduction, Outcome 3 Pre-quit reduction in cpd.

Analysis 6.4. Comparison 6 More structured vs less structured reduction, Outcome 4 Pre-quit tobacco withdrawal.

Analysis 7.1. Comparison 7 Additional behavioural SC components, Outcome 1 Abstinence.

Analysis 7.2. Comparison 7 Additional behavioural SC components, Outcome 2 Pre-quit reduction in cpd.

Analysis 8.1. Comparison 8 Behavioural reduction versus nicotine fading, Outcome 1 Abstinence.

Analysis 8.2. Comparison 8 Behavioural reduction versus nicotine fading, Outcome 2 Pre-quit reduction in cotinine.

Analysis 8.3. Comparison 8 Behavioural reduction versus nicotine fading, Outcome 3 Pre-quit tobacco withdrawal. $\quad \ldots \ldots \ldots . . . . . . . . . \quad 182$

Analysis 9.1. Comparison 9 Other comparisons, Outcome 1 Abstinence.

Analysis 9.2. Comparison 9 Other comparisons, Outcome 2 Pre-quit reduction in cpd.

Analysis 9.3. Comparison 9 Other comparisons, Outcome 3 Pre-quit reduction in CO.

Analysis 9.4. Comparison 9 Other comparisons, Outcome 4 Pre-quit tobacco withdrawal \& additional AE information. ........... 184 
[Intervention Review]

\section{Smoking reduction interventions for smoking cessation}

Nicola Lindson ${ }^{1}$, Elias Klemperer², Bosun Hong ${ }^{3}$, José M Ordóñez-Mena1 ${ }^{1}$, Paul Aveyard ${ }^{1}$

1Nuffield Department of Primary Care Health Sciences, University of Oxford, Oxford, UK. 2Departments of Psychological Sciences \& Psychiatry, University of Vermont, Burlington, VT, USA. ${ }^{3}$ Oral Surgery Department, Birmingham Dental Hospital, Birmingham, UK

Contact address: Nicola Lindson, Nuffield Department of Primary Care Health Sciences, University of Oxford, Radcliffe Observatory Quarter, Woodstock Road, Oxford, Oxfordshire, OX2 6GG, UK. nicola.lindson@phc.ox.ac.uk.

Editorial group: Cochrane Tobacco Addiction Group

Publication status and date: New, published in Issue 9, 2019.

Citation: Lindson N, Klemperer E, Hong B, Ordóñez-Mena JM, Aveyard P. Smoking reduction interventions for smoking cessation. Cochrane Database of Systematic Reviews 2019, Issue 9. Art. No.: CD013183. DOI: 10.1002/14651858.CD013183.pub2.

Copyright @ 2019 The Cochrane Collaboration. Published by John Wiley \& Sons, Ltd.

\section{A B S T R A C T}

\section{Background}

The standard way most people are advised to stop smoking is by quitting abruptly on a designated quit day. However, many people who smoke have tried to quit many times and may like to try an alternative method. Reducing smoking behaviour before quitting could be an alternative approach to cessation. However, before this method can be recommended it is important to ensure that abrupt quitting is not more effective than reducing to quit, and to determine whether there are ways to optimise reduction methods to increase the chances of cessation.

\section{Objectives}

To assess the effect of reduction-to-quit interventions on long-term smoking cessation.

\section{Search methods}

We searched the Cochrane Tobacco Addiction Group Specialised Register, MEDLINE, Embase and PsycINFO for studies, using the terms: cold turkey, schedul ${ }^{\star}$, cut $^{\star}$ down, cut-down, gradual ${ }^{\star}$, abrupt ${ }^{\star}$, fading, reduc ${ }^{\star}$, taper ${ }^{\star}$, controlled smoking and smoking reduction. We also searched trial registries to identify unpublished studies. Date of the most recent search: 29 October 2018.

\section{Selection criteria}

Randomised controlled trials in which people who smoked were advised to reduce their smoking consumption before quitting smoking altogether in at least one trial arm. This advice could be delivered using self-help materials or behavioural support, and provided alongside smoking cessation pharmacotherapies or not. We excluded trials that did not assess cessation as an outcome, with follow-up of less than six months, where participants spontaneously reduced without being advised to do so, where the goal of reduction was not to quit altogether, or where participants were advised to switch to cigarettes with lower nicotine levels without reducing the amount of cigarettes smoked or the length of time spent smoking. We also excluded trials carried out in pregnant women.

\section{Data collection and analysis}

We followed standard Cochrane methods. Smoking cessation was measured after at least six months, using the most rigorous definition available, on an intention-to-treat basis. We calculated risk ratios (RRs) and 95\% confidence intervals (Cls) for smoking cessation for each study, where possible. We grouped eligible studies according to the type of comparison (no smoking cessation treatment, abrupt quitting interventions, and other reduction-to-quit interventions) and carried out meta-analyses where appropriate, using a Mantel-Haenszel random-effects model. We also extracted data on quit attempts, pre-quit smoking reduction, adverse events (AEs), serious adverse events (SAEs) and nicotine withdrawal symptoms, and meta-analysed these where sufficient data were available. 


\section{Main results}

We identified 51 trials with 22,509 participants. Most recruited adults from the community using media or local advertising. People enrolled in the studies typically smoked an average of 23 cigarettes a day. We judged 18 of the studies to be at high risk of bias, but restricting the analysis only to the five studies at low or to the 28 studies at unclear risk of bias did not significantly alter results.

We identified very low-certainty evidence, limited by risk of bias, inconsistency and imprecision, comparing the effect of reduction-toquit interventions with no treatment on cessation rates (RR 1.74, 95\% Cl 0.90 to 3.38; $\mathrm{I}^{2}=45 \%$; 6 studies, 1599 participants). However, when comparing reduction-to-quit interventions with abrupt quitting (standard care) we found evidence that neither approach resulted in superior quit rates ( $R R 1.01,95 \% \mathrm{Cl} 0.87$ to $1.17 ; \mathrm{I}^{2}=29 \% ; 22$ studies, 9219 participants). We judged this estimate to be of moderate certainty, due to imprecision. Subgroup analysis provided some evidence $(P=0.01,12=77 \%)$ that reduction-to-quit interventions may result in more favourable quit rates than abrupt quitting if varenicline is used as a reduction aid. Our analysis comparing reduction using pharmacotherapy with reduction alone found low-certainty evidence, limited by inconsistency and imprecision, that reduction aided by pharmacotherapy resulted in higher quit rates (RR $1.68,95 \% \mathrm{Cl} 1.09$ to $2.58 ; \mathrm{I}^{2}=78 \%$; 11 studies, 8636 participants). However, a significant subgroup analysis $\left(\mathrm{P}<0.001, \mathrm{I}^{2}=80 \%\right.$ for subgroup differences) suggests that this may only be true when fast-acting NRT or varenicline are used (both moderate-certainty evidence) and not when nicotine patch, combination NRT or bupropion are used as an aid (all low- or very low-quality evidence). More evidence is likely to change the interpretation of the latter effects.

Although there was some evidence from within-study comparisons that behavioural support for reduction to quit resulted in higher quit rates than self-help resources alone, the relative efficacy of various other characteristics of reduction-to-quit interventions investigated through within- and between-study comparisons did not provide any evidence that they enhanced the success of reduction-to-quit interventions. Pre-quit AEs, SAEs and nicotine withdrawal symptoms were measured variably and infrequently across studies. There was some evidence that AEs occurred more frequently in studies that compared reduction using pharmacotherapy versus no pharmacotherapy; however, the AEs reported were mild and usual symptoms associated with NRT use. There was no clear evidence that the number of people reporting SAEs, or changes in withdrawal symptoms, differed between trial arms.

\section{Authors' conclusions}

There is moderate-certainty evidence that neither reduction-to-quit nor abrupt quitting interventions result in superior long-term quit rates when compared with one another. Evidence comparing the efficacy of reduction-to-quit interventions with no treatment was inconclusive and of low certainty. There is also low-certainty evidence to suggest that reduction-to-quit interventions may be more effective when pharmacotherapy is used as an aid, particularly fast-acting NRT or varenicline (moderate-certainty evidence). Evidence for any adverse effects of reduction-to-quit interventions was sparse, but available data suggested no excess of pre-quit SAEs or withdrawal symptoms. We downgraded the evidence across comparisons due to risk of bias, inconsistency and imprecision. Future research should aim to match any additional components of multicomponent reduction-to-quit interventions across study arms, so that the effect of reduction can be isolated. In particular, well-conducted, adequately-powered studies should focus on investigating the most effective features of reduction-to-quit interventions to maximise cessation rates.

\section{PLAIN LANGUAGE SUMMARY}

\section{Can people stop smoking by cutting down the amount they smoke first?}

\section{Background}

The standard way people are told to quit smoking is to smoke as normal until a quit day, when they stop using all cigarettes. However, many have tried this before and might like to try something new. Some people would just prefer to cut down the amount of cigarettes they smoke before quitting completely. Before healthcare services give people a choice of cutting down first or stopping all at once we need to find out whether cutting down helps as many people to stop smoking.

There are different ways that people could reduce the amount they smoke (for example, setting goals, lengthening the time between cigarette breaks) and some of these may work better than others. This review looks at whether cutting down before quitting helps people to stop smoking, and the best ways that people can cut down to help them stop completely.

\section{Study characteristics}

This review includes 51 studies of over 22,000 people who smoked tobacco. Most were adults, and people typically smoked at least 23 cigarettes a day at the start of the studies. All studies included at least one group of people who were asked to cut down their smoking and then quit tobacco smoking altogether. This group was compared to either a group who did not receive any treatment to stop smoking, a group who were asked to stop smoking all at once, or a group who were also asked to cut down their smoking in a different way. We did not include studies which asked people to cut down without quitting. Studies lasted for at least six months. The evidence is up to date to October 2018.

\section{Key results}


There was not enough information available to decide whether cutting down before quitting helped more people to stop smoking than no stop-smoking treatment. However, people who were asked to stop smoking all of their cigarettes at once were not more likely to quit than people who were asked to cut down their smoking before quitting. This suggests that asking people to cut down their smoking first may be a useful way to help people to stop smoking. People who cut down their smoking while using varenicline or a fast-acting form of nicotine replacement therapy (NRT), such as gum or lozenge, may be more likely to quit smoking than people who cut down their smoking without using a medicine to help them. Giving people face-to-face support to cut down their smoking may help more people to quit than if they are provided with self-help materials to cut down by themselves. There was not enough information available to decide whether other features of the cutting-down-to-quit intervention improved people's chances of stopping smoking.

We looked at whether being asked to cut down smoking before quitting resulted in negative effects, such as cigarette cravings, difficulty sleeping, low mood or irritability. Most studies did not provide information about this; more studies are therefore needed to answer this question.

\section{Quality of the evidence}

There is very low-quality evidence looking at whether cutting down smoking before quitting helps more people to quit smoking than no treatment. We rated the quality as very low, as there were problems with the design of studies, findings of studies were very different from one another, and not enough people took part, making it difficult to tell whether cutting down helps people to quit smoking. However, there is moderate-certainty evidence that cutting down before quitting may result in similar quit rates to quitting all at once, which suggests that cutting down may be a helpful approach. We rated this evidence as moderate because there is a chance that future studies may find that cutting down helps slightly more or slightly fewer people to quit than when people quit all at once. There is also moderate-quality evidence that people may be more likely to quit by cutting down first when they use a stop-smoking medicine like varenicline or a type of fast-acting NRT to help them. We rated this evidence as moderate certainty because there were not enough people taking part; more studies are needed. 
SUMMARY OF FINDINGS

\section{Summary of findings for the main comparison. Reduction to quit versus abrupt quitting for smoking cessation}

Reduction to quit versus abrupt quitting for smoking cessation

Patient or population: people who smoke

Setting: community; worksites; primary care and outpatient clinics; universities, high schools (Austria; China; Spain; Switzerland; UK; USA)

Intervention: reduction to quit

Comparison: abrupt quitting

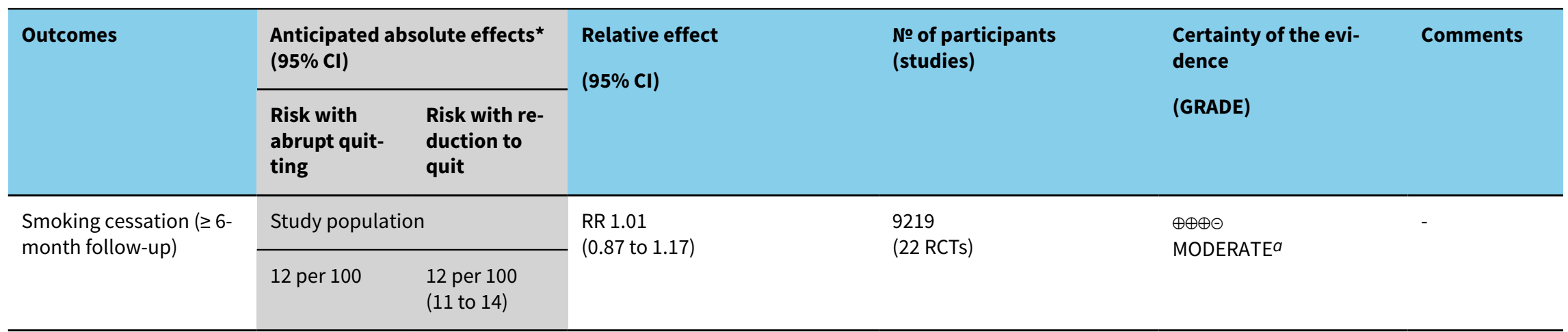

${ }^{\star}$ The risk in the intervention group (and its $95 \%$ confidence interval) is based on the assumed risk in the comparison group and the relative effect of the intervention (and its $95 \% \mathrm{Cl}$ )

CI: Confidence interval; RCT: randomised controlled trial; RR: Risk ratio

GRADE Working Group grades of evidence

High certainty: We are very confident that the true effect lies close to that of the estimate of the effect

Moderate certainty: We are moderately confident in the effect estimate: The true effect is likely to be close to the estimate of the effect, but there is a possibility that it is

substantially different

Low certainty: Our confidence in the effect estimate is limited: The true effect may be substantially different from the estimate of the effect

Very low certainty: We have very little confidence in the effect estimate: The true effect is likely to be substantially different from the estimate of effect

aDowngraded by one level due to imprecision: the $\mathrm{Cl}$ includes both clinically meaningful benefit and harm.

Summary of findings 2 . Reduction to quit versus no treatment for smoking cessation

Reduction to quit versus no treatment for smoking cessation

Patient or population: people who smoke 
Setting: community; primary care and outpatient clinics (China; Germany; USA)

Intervention: reduction to quit

Comparison: no smoking cessation treatment

\begin{tabular}{|c|c|c|c|c|c|c|}
\hline \multirow[t]{2}{*}{ Outcomes } & \multicolumn{2}{|c|}{$\begin{array}{l}\text { Anticipated absolute effects }{ }^{*} \\
(95 \% \mathrm{Cl})\end{array}$} & \multirow[t]{2}{*}{$\begin{array}{l}\text { Relative effect } \\
(95 \% \mathrm{CI})\end{array}$} & \multirow[t]{2}{*}{$\begin{array}{l}\text { № of participants } \\
\text { (studies) }\end{array}$} & \multirow{2}{*}{$\begin{array}{l}\text { Certainty of } \\
\text { the evidence } \\
\text { (GRADE) }\end{array}$} & \multirow[t]{2}{*}{ Comments } \\
\hline & $\begin{array}{l}\text { Risk with no } \\
\text { treatment }\end{array}$ & $\begin{array}{l}\text { Risk with re- } \\
\text { duction to } \\
\text { quit }\end{array}$ & & & & \\
\hline \multirow{2}{*}{$\begin{array}{l}\text { Smoking cessation ( } \geq 6 \text { month } \\
\text { follow-up) }\end{array}$} & \multicolumn{2}{|c|}{ Study population } & \multirow{2}{*}{$\begin{array}{l}\text { RR } 1.74 \\
\text { (0.90 to } 3.38)\end{array}$} & \multirow{2}{*}{$\begin{array}{l}1599 \\
\text { (6 RCTs) }\end{array}$} & \multirow{2}{*}{$\begin{array}{l}\oplus \ominus \ominus \ominus \\
\text { VERY LOW a,b,c }\end{array}$} & \multirow[t]{2}{*}{-} \\
\hline & 4 per 100 & $\begin{array}{l}6 \text { per } 100 \\
\text { ( } 3 \text { to } 12)\end{array}$ & & & & \\
\hline
\end{tabular}

${ }^{*}$ The risk in the intervention group (and its $95 \%$ confidence interval) is based on the assumed risk in the comparison group and the relative effect of the intervention (and its $95 \% \mathrm{Cl})$.

CI: Confidence interval; $\mathbf{R C T}$ : randomised controlled trial; $\mathbf{R R}$ : Risk ratio

\section{GRADE Working Group grades of evidence}

High certainty: We are very confident that the true effect lies close to that of the estimate of the effect

Moderate certainty: We are moderately confident in the effect estimate: The true effect is likely to be close to the estimate of the effect, but there is a possibility that it is substantially different

Low certainty: Our confidence in the effect estimate is limited: The true effect may be substantially different from the estimate of the effect

Very low certainty: We have very little confidence in the effect estimate: The true effect is likely to be substantially different from the estimate of effect

aDowngraded by one level due to risk of bias: we judged four of the six studies to be at high risk of bias and a further study at unclear risk.

bDowngraded by one level due to inconsistency: moderate unexplained heterogeneity detected $(12=45 \%)$.

cDowngraded by one level due to imprecision: there are few overall events and the $95 \% \mathrm{Cl}$ includes both the possibility of harm and appreciable benefit.

\section{Summary of findings 3. Reduction and pharmacotherapy versus reduction alone for smoking cessation}

Reduction and pharmacotherapy compared to reduction alone for smoking cessation

Patient or population: people who smoke

Setting: community; primary care (Australia; Canada; Czech Republic; Denmark; Egypt, Germany; Japan, Mexico, New Zealand; Switzerland; Taiwan; UK; USA)

Intervention: reduction to quit aided by pharmacotherapy

Comparison: reduction to quit alone (placebo or no pharmacotherapy) 


\begin{tabular}{|c|c|c|c|c|c|c|}
\hline \multirow[t]{2}{*}{ Outcomes } & \multicolumn{2}{|c|}{$\begin{array}{l}\text { Anticipated absolute effects }{ }^{*} \\
(95 \% \mathrm{Cl})\end{array}$} & \multirow[t]{2}{*}{$\begin{array}{l}\text { Relative effect } \\
(95 \% \mathrm{Cl})\end{array}$} & \multirow[t]{2}{*}{$\begin{array}{l}\text { № of partici- } \\
\text { pants } \\
\text { (studies) }\end{array}$} & \multirow[t]{2}{*}{$\begin{array}{l}\text { Certainty of the } \\
\text { evidence } \\
\text { (GRADE) }\end{array}$} & \multirow[t]{2}{*}{ Comments } \\
\hline & $\begin{array}{l}\text { Risk with } \\
\text { placebo/n } \\
\text { pharma- } \\
\text { cotherapy }\end{array}$ & $\begin{array}{l}\text { Risk with } \\
\text { pharma- } \\
\text { cotherapy }\end{array}$ & & & & \\
\hline \multirow{2}{*}{$\begin{array}{l}\text { Main analysis (all pharmacotherapy): smoking } \\
\text { cessation ( } \geq 6 \text { month follow-up) }\end{array}$} & \multicolumn{2}{|c|}{ Study population } & \multirow{2}{*}{$\begin{array}{l}\text { RR } 1.68 \\
\text { (1.09 to } 2.58)\end{array}$} & \multirow{2}{*}{$\begin{array}{l}8636 \\
(11 \mathrm{RCTs})\end{array}$} & \multirow{2}{*}{$\begin{array}{l}\oplus \oplus \ominus \ominus \\
\text { LOWa,b,c }\end{array}$} & \multirow{2}{*}{$\begin{array}{l}\text { The rows be- } \\
\text { low are sub- } \\
\text { groups of this } \\
\text { main analysis. }\end{array}$} \\
\hline & 5 per 100 & $\begin{array}{l}8 \text { per } 100 \\
(5 \text { to } 13)\end{array}$ & & & & \\
\hline \multirow{2}{*}{$\begin{array}{l}\text { Subgroup: combination NRT; smoking cessation } \\
\text { ( } \geq 6 \text { month follow-up) }\end{array}$} & \multicolumn{2}{|c|}{ Study population } & \multirow{2}{*}{$\begin{array}{l}\text { RR } 1.02 \\
\text { (0.61 to } 1.69)\end{array}$} & \multirow{2}{*}{$\begin{array}{l}1124 \\
\text { (3 RCTs) }\end{array}$} & \multirow{2}{*}{$\begin{array}{l}\oplus \oplus \ominus \ominus \\
\text { LOWd,e,f }\end{array}$} & \multirow[t]{2}{*}{-} \\
\hline & 15 per 100 & $\begin{array}{l}15 \text { per } 100 \\
(9 \text { to } 25)\end{array}$ & & & & \\
\hline \multirow{2}{*}{$\begin{array}{l}\text { Subgroup: nicotine patch; smoking cessation ( } \geq 6 \\
\text { month follow-up) }\end{array}$} & \multicolumn{2}{|c|}{ Study population } & \multirow{2}{*}{$\begin{array}{l}\text { RR } 0.34 \\
\text { (0.02 to } 5.31)\end{array}$} & \multirow{2}{*}{$\begin{array}{l}85 \\
(1 \mathrm{RCT})\end{array}$} & \multirow{2}{*}{$\begin{array}{l}\oplus \Theta \Theta \Theta \\
\text { VERY LOWg,h }\end{array}$} & \multirow[t]{2}{*}{-} \\
\hline & 15 per 100 & $\begin{array}{l}5 \text { per } 100 \\
(0 \text { to } 80)\end{array}$ & & & & \\
\hline \multirow{2}{*}{$\begin{array}{l}\text { Subgroup: fast-acting NRT only; smoking cessa- } \\
\text { tion ( } \geq 6 \text { month follow-up) }\end{array}$} & \multicolumn{2}{|c|}{ Study population } & \multirow{2}{*}{$\begin{array}{l}\text { RR } 2.56 \\
\text { (1.93 to } 3.39)\end{array}$} & \multirow{2}{*}{$\begin{array}{l}5323 \\
(7 \mathrm{RCTs})\end{array}$} & \multirow{2}{*}{$\begin{array}{l}\oplus \oplus \oplus \ominus \\
\text { MODERATEi }\end{array}$} & \multirow[t]{2}{*}{-} \\
\hline & 2 per 100 & $\begin{array}{l}6 \text { per } 100 \\
(5 \text { to } 8)\end{array}$ & & & & \\
\hline \multirow{2}{*}{$\begin{array}{l}\text { Subgroup: varenicline only; smoking cessation ( } \geq \\
6 \text { month follow-up) }\end{array}$} & \multicolumn{2}{|c|}{ Study population } & \multirow{2}{*}{$\begin{array}{l}\text { RR } 3.99 \\
\text { ( } 2.93 \text { to } 5.44)\end{array}$} & \multirow{2}{*}{$\begin{array}{l}1510 \\
(1 \mathrm{RCT})\end{array}$} & \multirow{2}{*}{$\begin{array}{l}\oplus \oplus \oplus \ominus \\
\text { MODERATEi }\end{array}$} & \multirow[t]{2}{*}{-} \\
\hline & 6 per 100 & $\begin{array}{l}24 \text { per } 100 \\
\text { (18 to } 33)\end{array}$ & & & & \\
\hline \multirow{2}{*}{$\begin{array}{l}\text { Subgroup: bupropion only; smoking cessation ( } \geq \\
6 \text { month follow-up) }\end{array}$} & \multicolumn{2}{|c|}{ Study population } & \multirow{2}{*}{$\begin{array}{l}\text { RR } 1.27 \\
\text { (0.67 to } 2.40)\end{array}$} & 594 & $\oplus \oplus \ominus \ominus$ & - \\
\hline & 5 per 100 & $\begin{array}{l}7 \text { per } 100 \\
(4 \text { to } 13)\end{array}$ & & & & \\
\hline
\end{tabular}

${ }^{\star}$ The risk in the intervention group (and its $95 \%$ confidence interval) is based on the assumed risk in the comparison group and the relative effect of the intervention (and its $95 \% \mathrm{Cl}$ ).

Cl: Confidence interval; RCT: randomised controlled trial; RR: Risk ratio 
High certainty: We are very confident that the true effect lies close to that of the estimate of the effect

Moderate certainty: We are moderately confident in the effect estimate: The true effect is likely to be close to the estimate of the effect, but there is a possibility that it is substantially different

Low certainty: Our confidence in the effect estimate is limited: The true effect may be substantially different from the estimate of the effect

Very low certainty: We have very little confidence in the effect estimate: The true effect is likely to be substantially different from the estimate of effect

aWe did not downgrade due to risk of bias: A sensitivity analysis removing studies judged to be at high risk of bias did not change our interpretation of the effect.

bDowngraded by one level due to inconsistency: substantial heterogeneity was detected $(12=78 \%)$. A subgroup analysis grouping by type of pharmacotherapy used explained a small amount of this, but moderate heterogeneity remained unexplained.

'Downgraded by one level due to imprecision: the Cls of the absolute effect indicate potentially no benefit of pharmacotherapy, whereas the upper limit indicates the potential for a clinical benefit.

dDowngraded by one level due to risk of bias: we rated two of the three studies at high risk of bias, due to the use of an unmatched placebo. However, removal of these studies did not change the interpretation of the effect.

eWe did not downgrade, due to inconsistency despite an $\mathrm{I}^{2}$ of $44 \%$, as there was a high degree of $\mathrm{Cl}$ overlap.

fDowngraded by one level due to imprecision: the event rate is low and the Cls of the effect estimate incorporate clinically relevant potential benefit and harm of the intervention. gDowngraded by one level due to risk of bias: the only study in this comparison was at high risk of bias.

hDowngraded by two levels due to imprecision: the event rate is very low $(n=6)$ and the $\mathrm{Cl}$ of the effect estimate indicates considerable harm as well as benefit.

iDowngraded by one level due to imprecision: the overall number of events was low $(<300)$.

jDowngraded by one level due to risk of bias: we rated the only included study at high risk of attrition bias, due to high rates of dropout. 


\section{B A C K G R O U N D}

\section{Description of the condition}

Tobacco use is one of the leading causes of preventable illness and death worldwide, accounting for over 7 million deaths annually (GBD 2015 Risk Factors Collaborators 2016). Extrapolation based on current smoking trends suggests that without widespread quitting approximately 400 million tobacco-related deaths will occur between 2010 and 2050, mostly among current smokers (Jha 2011). However, most smokers would like to stop. In 2015 a survey by the Centers for Disease Control and Prevention (CDC 2017) found that $68 \%$ of smokers in the USA would like to quit smoking completely. However, quitting smoking is very difficult, with only a minority of smokers who try to quit going on to be successful (Hughes 2004a). An analysis that attempted to accurately estimate the number of quit attempts needed for a smoker to quit found that it may take 30 or more attempts before a smoker is successful (Chaiton 2016). Providing a range of successful and appealing quitting options may encourage smokers, most of whom have tried to quit repeatedly, to keep on trying.

\section{Description of the intervention}

Historically, the standard way to stop smoking has been to quit abruptly, and this is the primary approach recommended by both the UK's (NICE 2018) and USA's (Fiore 2008) clinical guidance. This means that a person smokes as normal until an agreed quit day, and from that point forward they try to abstain and avoid any smoking whatsoever. However, an alternative method is to quit gradually, by reducing the amount of tobacco smoked before quitting completely. Such gradual reduction methods, when used as a means of achieving cessation, typically have a quit day as in abrupt cessation. The key difference is that smokers aim to reduce smoking prior to this day. There are many potential ways that smokers could go about this reduction, for example:

- setting a particular time period during which to reduce before quitting completely;

- setting goals to reduce by a certain number of cigarettes a day;

- reducing the time periods in the day when smoking occurs (rather than reducing the number of cigarettes);

- smoking on a planned schedule in which the time between cigarettes gradually lengthens;

- using pharmacotherapy, such as nicotine replacement therapy (NRT), or an electronic cigarette to discourage smoking or replace cigarettes not smoked;

- setting out with the intention to reduce smoking before quitting, without a specific plan of how to go about it.

\section{How the intervention might work}

There are a number of ways that reducing the number of cigarettes smoked prior to total abstinence might help a smoker give up completely. Firstly, as the dose of nicotine received by the individual each day is reduced, drug dependence and therefore craving may reduce in response (Lindson 2012). Another potential mechanism is 'shaping', an operant conditioning procedure whereby through making successive approximations of the target behaviour that are positively reinforced (gradually cutting down the number of cigarettes smoked), the desired behaviour (abstinence) is eventually achieved (Skinner 1953). The third is the cognitive psychology principle that completing a step toward a goal (reducing smoking) in- creases self-efficacy, which increases the likelihood that the goal (abstinence) will be achieved (Bandura 1977). The fourth is the classical and operant conditioning principle that reducing the frequency of a behaviour decreases the association with environmental cues, which in turn weakens the urge to partake in that behaviour when those cues are present (Bouton 1991). Finally, reducing may simply provide a goal that is more in line with the smoker's current behaviour than complete abstinence, and it may therefore appear more achievable and enhance motivation to quit. This appears to be supported by the popularity of the approach amongst smokers. Surveys in both the UK and the USA indicate that a substantial proportion of smokers attempting to quit in the general population choose to do so by cutting down their smoking first. West 2006 found that $40 \%$ of UK quit attempts involved cutting down first, and a random sample of smokers in the USA showed that nearly half of smokers planning to quit would choose reduction over abrupt cessation (Shiffman 2007). There was little interest among these smokers in reduction as an end in itself, but only as a means to abstinence.

The standard assumption of smoking cessation treatment is that cessation begins on a quit day and that cutting down prior to quitting is not advised. This is based on nicotine addiction theory, which posits that the user has impaired control over their drug use, and that it would therefore be difficult for them to control their usage in any way, e.g. by reducing. Nicotine addiction theory also proposes that with reduction each remaining cigarette will become more rewarding and harder to give up, and that the smoker will suffer a loss of motivation, meaning they may be less likely to make a quit attempt and achieve total abstinence (Denning 2002; Hajek 1989). However, medication to reduce withdrawal, such as NRT or electronic cigarettes, could be used to counteract this effect, and NRT has successfully been used to do so in smokers who have chosen to reduce their smoking, but are not yet ready to quit (McRobbie 2006; Wang 2008). A number of literature reviews have found evidence to suggest that smoking reduction is associated with future cessation (Fagerström 2005; Hughes 2006), and this may be an approach that is particularly attractive for populations who find it hard to quit, such as people with mental health problems or other substance abuse issues.

\section{Why it is important to do this review}

Although the UK's (NICE 2018) and USA's (Fiore 2008) national guidelines for smoking cessation do not recommend reducing smoking before quitting as a first step for smoking cessation treatment, both acknowledge that the evidence for this approach is unclear. The field would therefore benefit from further research to establish whether it could be used as a successful, alternative intervention to abrupt quitting. Surveys have been carried out across England and Wales (Garnett 2019; West 2012) and the UK, USA, Canada and Australia (Cheong 2007), investigating the success of quit attempts when smokers choose to reduce cigarettes smoked with the aim of quitting completely. Both of these observational studies found that people who chose to quit abruptly were almost twice as successful as those who chose to quit gradually. However, this could be because those who chose to quit gradually were less motivated to quit (Peters 2007), had found it harder to quit in the past, and/or did not use a successful treatment service, intervention or reduction method to quit. Unlike abrupt quitting, which allows for very little variation in method, participants could potentially have used a wide range of gradual quitting techniques, rang- 
ing from no structure, no reduction goals and no set quit day, to highly structured, with set reduction goals and a target quit day to work toward. It is reasonable to assume that different approaches to reducing may be more or less likely to result in abstinence, and this variation may have influenced success rates.

The aim of this review is to investigate the potential success of reducing smoking as a precursor to stopping smoking completely, by answering the following questions.

- How successful are reduction-to-quit interventions in comparison to no smoking-cessation treatment or advice?

- How successful are reduction-to-quit interventions in comparison to abrupt quitting interventions?

- Which method of reducing smoking prior to quitting results in the highest quit rates?

The first question is important, as many smoking cessation services currently recommend abrupt cessation for all quit attempts (first or repeated). However, alternative methods might give renewed hope and encourage cessation in those who have given this up as impossible. If gradual cessation results in greater quit rates than no treatment at all then it could be offered by cessation services or recommended to the general population, as a new way to quit for those who are not motivated to try quitting abruptly. Given that behavioural support and pharmacotherapy increase the likelihood of achieving abstinence (Hartmann-Boyce 2018; Lancaster 2017; Stead 2017), encouraging more people to use cessation services would have public health benefits. The second question is important, as there may be people who want to quit smoking who do not mind whether they attempt to do so gradually or abruptly. It is important to give these people the best possible chance of quitting by advising them to use the method that the evidence suggests results in the highest quit rates. Finally, if the answers to the first and second questions suggest that reduction to quit may be a useful approach to smoking cessation for some or all smokers, then it would be valuable to answer the third question to inform the application of these interventions.

Please note that this review is an update of a previously published Cochrane review (Lindson 2010; Lindson-Hawley 2012). The original review focused solely on trials comparing smoking reduction-to-quit interventions with abrupt-quitting interventions. We have decided to widen the scope of the review to give a clearer overall view of the literature in this area.

Studies that investigate smoking reduction, where quitting is not the final aim of the intervention, are covered in a separate Cochrane Review of smoking harm reduction approaches (Lindson-Hawley 2016a).

\section{OB JECTIVES}

- To assess the effect of reduction-to-quit interventions on longterm smoking cessation

\section{Secondary objectives}

- To assess the proportion of participants who make quit attempts and quantify the reduction that occurs as a result of reduction-to-quit interventions
- To assess whether the efficacy of reduction-to-quit interventions is moderated by baseline motivation to quit, self-efficacy or preference for gradual versus abrupt cessation

- To investigate any adverse effects of reduction-to-quit interventions (including adverse events)

We assessed all of these objectives by comparing reduction-to-quit interventions with no smoking-cessation treatment, with abruptquitting interventions and with other reduction-to-quit interventions.

\section{METHODS}

\section{Criteria for considering studies for this review \\ Types of studies}

Randomised controlled trials (RCTs), including cluster-RCTs.

\section{Types of participants}

Cigarette smokers of any age willing to enrol in a smoking cessation trial. We excluded studies in pregnant smokers, as these are covered by Chamberlain 2017.

\section{Types of interventions}

This review includes interventions consisting of instruction/advice/support for participants to reduce the number of cigarettes they were smoking, where an ultimate goal of complete tobacco cessation was emphasised. Interventions were eligible if they advised participants to switch to another nicotine-containing product, as long as this product did not contain tobacco, i.e. NRT or electronic cigarettes. We did not include trials where participants spontaneously reduced before quitting without being advised to do so, or trials testing interventions that aimed to reduce smoking without advising participants to quit altogether. We also excluded trials that advised participants to switch to cigarettes with lower nicotine levels, without also advising them to reduce the number of cigarettes they smoked or the length of time they spent smoking. The latter two types of trial are covered by the Cochrane Review of tobacco harm reduction approaches (Lindson-Hawley 2016a).

Eligible interventions include any amount of behavioural support, and could also include concomitant pharmacotherapy or devices to support reduction or cessation.

\section{Comparators}

We include trials that compare the reduction intervention with any of the following comparators.

- No smoking-cessation treatment or advice;

- Abrupt quitting interventions: any advice to stop smoking abruptly without prior reduction. If advice to reduce smoking behaviour was not explicitly stated then we judged the intervention to be 'abrupt'. Abrupt interventions could include any amount of behavioural support, pharmacotherapy or quitting devices;

- Another reduction-to-quit intervention, regardless of the amount of behavioural support, pharmacotherapy or use of quitting devices.

There was no requirement for the level, nature or amount of intervention support or pharmacotherapy provided to be matched 
between trial arms, as we wished to review all of the evidence on reduction-to-quit interventions. However, we tested the potential impact of these factors using subgroup and sensitivity analyses, as described below (Subgroup analysis and investigation of heterogeneity; Sensitivity analysis).

\section{Types of outcome measures}

\section{Primary outcomes}

- Smoking abstinence at long-term follow-up (dichotomous). To be eligible for inclusion, studies had to measure follow-up at least six months from the start of the intervention. We excluded studies with abstinence measured at less than six months' follow-up.

In trials with more than one measure of abstinence, we preferred the measure with the longest follow-up and the strictest criteria, in line with the Russell Standard (West 2005). We used prolonged or continuous abstinence over point prevalence abstinence, and biochemically-validated abstinence, such as exhaled carbon monoxide (CO), over self-report. We favoured biochemically-validated point prevalence abstinence over self-reported continuous or prolonged abstinence.

\section{Secondary outcomes}

- Reduction in smoking behaviour between baseline and quit day/ end of the reduction period (dichotomous or continuous, or both) using measures defined by study authors. This could be measured as reduction in cigarettes per day (cpd) or reduction in a biomarker of smoking behaviour, such as exhaled CO, cotinine, anabasine, anatabine. We did not use cotinine as a measure of smoking reduction where a reduction aid containing nicotine was used (e.g. NRT, electronic cigarettes) prior to quit day, as this would be expected to impact on the levels detected. Reduction could be defined using a continuous measure (such as reduction in number of $\mathrm{cpd}$ ), or using a dichotomous measure (such as less than $50 \%$ reduction in cpd versus $50 \%$ or more reduction in cpd). We assessed this outcome to investigate whether participants who were advised to reduce their smoking actually did so, and whether they reduced more than people allocated to comparison interventions.

- Proportion of participants who made a quit attempt (dichotomous). We assessed this outcome to investigate whether reduction interventions reduce the likelihood of smokers making a quit attempt. We used authors' own definition of a quit attempt (such as at least 24 hours of abstinence), and this varied somewhat across studies.

- Proportions of participants who reported adverse events (including serious adverse events) occurring up to the smoking quit day (dichotomous). Where reported, we also extracted the total numbers of adverse events reported in this period (as more than one may have occurred per participant). We also extracted any measures taken of nicotine withdrawal symptoms during the pre-quit period, as these are common adverse effects of quitting smoking. We only reported adverse effects that occurred prior to quitting, as these are the effects most likely to have occurred in response to the smoking reduction intervention. Adverse events associated with smoking-cessation pharmacotherapy use are investigated in separate Cochrane Reviews of these therapies (Cahill 2016; Hartmann-Boyce 2018; Hughes 2014).

\section{Search methods for identification of studies}

\section{Electronic searches}

The evidence in this version of the review is up to date to 29 October 2018.

We searched the Cochrane Tobacco Addiction Review Group Specialised Register, which has been developed from electronic searches of MEDLINE, Embase and PsycINFO, together with handsearching of specialist journals, conference proceedings and reference lists of previous trials and overviews. At the time of the search, the Register included the results of searches of the Cochrane Central Register of Controlled trials (CENTRAL), issue 1, 2018; MEDLINE (via OVID) to update 20181026; Embase (via OVID) to week 201845; PsycINFO (via OVID) to update 20181022. See the Cochrane Tobacco Addiction Group website for full search strategies and a list of other resources searched. We searched the Specialised Register using the following terms: Cold turkey, schedul* ${ }^{\star}$ Cut ${ }^{\star}$ down, cutdown, Gradual ${ }^{\star}$, abrupt ${ }^{\star}$, fading, reduc ${ }^{\star}$, taper ${ }^{\star}$, controlled smoking, smoking reduction. See Appendix 1 for the complete search strategy.

We also searched MEDLINE, Embase and PsycINFO from inception, using the following topic-specific terms, combined with the terms used for the regular searches of MEDLINE, Embase and PsycINFO to identify trials of tobacco addiction interventions for the Tobacco Addiction Review Group Specialised Register (see Appendix 2; Appendix 3 and Appendix 4 for full search strategies):

- cold turkey.mp

- (schedul $^{\star}$ adj3 smok smp $^{\star}$.mp

- (cut ${ }^{\star}$ down or cut-down).mp

- ((\{Gradual ${ }^{\star}$ or abrupt $\left.\left.{ }^{\star}\right\}\right)$ adj3 (reduc or quit $^{\star}$ or stop ${ }^{\star}$ or abstin ${ }^{\star}$ or abstain ${ }^{\star}$ or cessat $\left.\left.{ }^{\star}\right)\right) . m p$

- fading.mp

- taper $^{\star} . \mathrm{mp}$

- (controlled adj smoking).mp

- Smoking reduction/ or smoking reduction.mp

( $\mathrm{mp}=$ title, original title, abstract, name of substance word, subject heading word)

\section{Searching other resources}

We searched the US National Library of Medicine's trial registry (clinicaltrials.gov) and the World Health Organization's clinical trials search portal (www.who.int/trialsearch) from inception to identify any eligible ongoing studies. We contacted the authors of known unpublished trials.

\section{Data collection and analysis}

\section{Selection of studies}

Two review authors (from $\mathrm{BH}, \mathrm{EK}, \mathrm{NL}$ ) independently checked the titles and abstracts of studies generated by the search strategy for relevance. We resolved any disagreements through discussion with a third review author. We obtained full-text versions of papers thought potentially relevant at this stage. Two review authors (from $\mathrm{BH}, \mathrm{EK}, \mathrm{NL}$ ) then independently assessed the full-text trial reports for inclusion in the review. We resolved any disagreements through discussion with a third review author. We screened and included 
studies reported in any language, and had non-English language papers translated.

\section{Data extraction and management}

For each included trial two review authors (from BH, EK, JMOM, $\mathrm{NL}, \mathrm{PA}$ ) independently extracted data. Authors then cross-checked this information between themselves, and resolved disagreements through discussion. We extracted the following information:

- Author

- Date and country of publication

- Study design

- Location and setting

- Recruitment method

- Summary of key study participant characteristics, including cigarettes smoked per day, nicotine dependence

- Baseline measure of motivation to quit

- Baseline measure of self-efficacy

- Participant preference for gradual or abrupt cessation at enrolment

- Summary of intervention and control condition methods, including any use of pharmacotherapy or other quitting aid (such as, electronic cigarettes), length of treatment, and the amount of smoking reduction advised prior to quit day

- Intervention provider

- Number of participants in each trial arm

- Definition of smoking cessation used

- Smoking cessation outcomes

- Type of biochemical validation (if any)

- Definition of adverse events used

- Numbers and proportions of participants who reported adverse events

- Total numbers of adverse events reported

- Measures of withdrawal used

- Withdrawal symptoms

- Definition of quit attempt used

- Number and proportions of participants who made a quit attempt

- Measure of reduction in smoking behaviour used

- Pre-quit smoking reduction

- Loss to follow-up

- Assessment time points

- Results of any moderator analysis investigating baseline motivation to quit, cigarettes per day, self-efficacy, or preference for gradual versus abrupt cessation

- Risk of bias in the domains specified below

- Funding source

- Author declarations of interest

- Additional comments

\section{Assessment of risk of bias in included studies}

Two review authors (from BH, EK, JMOM, NL, PA) independently assessed the risks of bias for each included study, following the approach recommended in the Cochrane Handbook for Systematic Reviews of Interventions (Cochrane Handbook; Higgins 2017). For each trial, we assessed the following domains: random sequence gen- eration; allocation concealment; blinding of outcome assessment; incomplete outcome data; and other sources of bias. Specific 'Risk of bias' guidance developed by the Cochrane Tobacco Addiction Group to assess smoking cessation trials states that performance bias (relating to the blinding of participants and providers) should not be assessed for behavioural interventions, as it is impossible to blind people to these types of interventions. We therefore only assessed performance bias for the subset of studies that compared smoking reduction carried out in addition to a pharmacological aid versus reduction carried out alongside a placebo or no pharmacotherapy.

Each review author recorded information in trial reports for each relevant domain and then assessed each domain as being at low, high, or unclear risk of bias. We resolved disagreements through discussion with a third review author.

\section{Measures of treatment effect}

We compared quit rates (primary outcome), smoking reduction, and the numbers and proportions of people making a quit attempt and reporting adverse events (secondary outcomes) between intervention and comparator groups for each study.

We calculated quit rates and number of participants making a quit attempt on an intention-to-treat basis, including all participants originally randomised to a trial arm. We treated participants lost to follow-up as relapsed. Dichotomous smoking reduction outcomes were also based on all participants randomised, with participants lost to follow-up assumed not to have changed their smoking behaviour from baseline. We calculated rates of participants reporting adverse events using the denominator provided by individual study papers for that outcome. We used risk ratios (RRs) and 95\% confidence intervals (Cls) as the summary statistics for quit rates, quit attempts, adverse event rates, and for dichotomous measures of reduction, for each study.

Where studies reported both dichotomous and continuous measures of smoking reduction we extracted both. For continuous measures, we calculated the mean differences (MDs) and 95\% Cls in the change in smoking consumption between baseline and quit day, where possible. We calculated change in consumption as the mean outcome at baseline minus the mean outcome at the time closest to quit day, resulting in a positive estimate if reduction occurred, and a negative estimate if an increase occurred. Numbers of participants contributing to analyses were based on the numbers reported to contribute to that outcome in individual study reports. However, where this was not reported or unclear, we used the total numbers randomised to each group.

\section{Unit of analysis issues}

In the case of cluster-randomised controlled trials, where available, we extracted a direct estimate of the required effect from an analysis that properly accounted for the cluster design. Where such data were unavailable, a statistician (JMOM) assessed the likely effect of clustering and whether adjustment was necessary.

In the case of trials with multiple arms, we combined all relevant experimental intervention groups of the study into a single group, and combined all relevant control intervention groups into a single control group, where we deemed this possible and appropriate to the structure of the analysis. 


\section{Dealing with missing data}

Where primary outcome data were missing we contacted the authors for clarification. For continuous reduction outcomes many studies did not report the standard deviation (SD) of the mean change (in cpd, CO or cotinine) from baseline to quit day. We estimated this using statistical methods recommended in the Cochrane Handbook (Higgins 2011). In a few cases, where estimation was not possible and the SD was reported at baseline but not at follow-up, we assumed the SD at follow-up to be the same as at baseline.

\section{Assessment of heterogeneity}

In order to assess whether it was appropriate to pool studies and conduct meta-analyses, we assessed the characteristics of included studies to identify any clinical or methodological heterogeneity. Where we deemed studies homogeneous enough to be meaningfully combined, we conducted a meta-analysis, and we assessed statistical heterogeneity using the $\mathrm{I}^{2}$ statistic. We deemed an $\mathrm{I}^{2}$ of greater than $50 \%$ to be substantial heterogeneity. Where $1^{2}$ exceed ed $80 \%$ we did not report a pooled point estimate, as it is unlikely that this would be useful or informative.

We conducted the subgroup and sensitivity analyses described below (Subgroup analysis and investigation of heterogeneity; Sensitivity analysis) to investigate any potential causes of observed heterogeneity, where there were enough data included in an analysis to draw meaningful conclusions.

\section{Assessment of reporting biases}

We assessed reporting bias using funnel plots for comparisons where we identified and analysed abstinence rates from at least 10 studies (reduction to quit versus abrupt quitting; reduction with pharmacotherapy versus reduction alone). Funnel plots illustrate the relationship between the effect estimates from individual studies against their size or precision. The greater the degree of asymmetry, the greater the risk of reporting bias.

\section{Data synthesis}

We provided a narrative summary of included studies and, where possible, conducted meta-analyses of abstinence, quit attempts, smoking reduction, and adverse event outcomes. This review includes three comparisons: 1) reduction interventions versus no treatment; 2) reduction interventions versus abrupt interventions; 3) reduction interventions versus other reduction interventions. The third 'reduction interventions versus reduction interventions' comparison was split into finer subgroups as follows:

- whether or not reduction was aided by pharmacotherapy

- the modality of support (behavioural versus self-help)

- the length of the smoking reduction period (e.g. one week versus one month)

- whether advice to reduce was structured versus unstructured

- the intensity of behavioural support provided and whether additional behavioural support components were offered alongside the smoking reduction advice (e.g. additional sessions of behavioural smoking cessation support or additional medication adherence counselling)

- other comparisons (explored in only a single study)
We grouped studies by comparison and only pooled for metaanalyses within these separate groupings, i.e. reduction versus abrupt studies were not pooled with reduction versus no-treatment studies.

The primary outcome of abstinence and the secondary outcomes of quit attempts and number of participants experiencing adverse event outcomes all provided dichotomous data, so in these cases we combined RRs from individual studies using Mantel-Haenszel random-effects methods, to calculate pooled overall risk ratios with $95 \%$ Cls. We specified a priori that it would be most appropriate to pool data using a random-effects approach, as behavioural interventions and comparators varied substantially between studies. Where only total numbers of adverse events or measures of withdrawal were reported, or where they were reported alongside numbers of participants experiencing adverse events, we tabulated them narratively.

For the secondary outcome of smoking reduction, the measures used across studies were diverse, making overall pooling unfeasible. Where available, we pooled continuous data using random-effects, inverse variance methods to generate MDs and 95\% Cls; and pooled dichotomous data separately using a Mantel-Haenszel random-effects model to generate RRs and $95 \% \mathrm{Cls}$.

To satisfy our third objective ('to assess whether the efficacy of reduction to quit interventions is moderated by baseline motivation to quit, self-efficacy or preference for gradual versus abrupt cessation'), we tabulated the results of any within-study analyses investigating these moderators narratively.

\section{Subgroup analysis and investigation of heterogeneity}

Where the nature of a comparison and the amount of data contributing to that comparison potentially enabled us to draw meaningful conclusions, we conducted subgroup analyses for the primary outcome (smoking abstinence). We conducted the following analyses for the 'reduction to quit versus abrupt quitting' comparison only:

- grouped by whether or not participants had a set quit date in the reduction group;

- grouped by whether or not participants in the reduction group were advised to reduce the number of cigarettes they smoked (cpd method) or the amount of time they smoked (smoke-free periods (sfp) method)

- grouped by whether or not participants were given structured instructions on ways to reduce their smoking, e.g. by increasing the time between cigarettes, not smoking at home, stopping smoking particular cigarettes first;

- grouped according to the length of the smoking reduction period advised before the quit day;

- grouped according to the amount of reduction (\%) advised before the quit day in the reduction-to-quit intervention group.

We carried out the following analysis for the 'reduction to quit versus abrupt quitting' and the 'reduction with pharmacotherapy versus reduction alone' comparisons:

- grouped by whether or not pharmacotherapy was used to aid reduction, and what type was used (for example, NRT, varenicline, bupropion). 
It was impossible to carry out the following prespecified subgroup analysis as the required details were not reported with enough consistency to group studies into the appropriate categories:

- grouping according to the amount of reduction actually achieved (rather than advised) before the quit day in the reduction-to-quit intervention group (for example, no reduction versus a $25 \%$ reduction in cpd versus a $50 \%$ reduction in cpd versus a $75 \%$ reduction in cpd).

\section{Sensitivity analysis}

We conducted the following sensitivity analysis, where possible, across all comparisons for the primary (abstinence) outcome:

- removing studies deemed to be at high risk of bias

We conducted the following sensitivity analyses for the primary outcome (abstinence), for the reduction versus abrupt quitting comparison only. This was the only comparison for which there were enough studies to make these analyses both appropriate and feasible:

- removing studies where participants in the reduction arm received pharmacotherapy to aid smoking cessation but no pharmacotherapy was offered at any point pre- or post-quit in the abrupt quitting arm. This was to try and remove any effect of simply offering pharmacotherapy;

- removing studies where the overall intensity or contact time of the intervention and comparator programmes (pre- and postquit combined) were unmatched. We were primarily interested in any pre-quit intervention difference between the reduction and abrupt quitting arms. However in many studies the quit day was not well defined, making it impossible to clearly judge when a mismatch in support occurred (i.e. pre- or post-quit) between trial arms. Thus, this sensitivity analysis tested overall differences (pre- and post- combined) in the intensity of support provided, because this may have impacted our interpretation of differences between pre-quit interventions.

\section{'Summary of findings' table}

Following standard Cochrane methods (Schünemann 2017), we created a 'Summary of findings' table for the primary outcome (smoking abstinence), for each of the following comparisons: 1) reduction versus no treatment ; 2 ) reduction versus abrupt cessation; 3) reduction with pharmacotherapy versus reduction alone. Also following standard Cochrane methodology, we used the five GRADE considerations (risk of bias, inconsistency, imprecision, indirectness and publication bias) to assess the certainty of the body of evidence for the abstinence outcome for each comparison, and to draw conclusions about the quality of evidence within the text of the review.

\section{RES U L T S}

\section{Description of studies}

See Characteristics of included studies; Characteristics of excluded studies; Characteristics of studies awaiting classification; Characteristics of ongoing studies.

\section{Results of the search}

Our searches resulted in 3489 records. After duplicates were removed, 1944 records remained for title and abstract screening. We ruled out 1682 records at this stage, leaving 262 for full-text screening. We identified 51 completed studies, eight ongoing studies, and seven studies awaiting classification, and excluded 196 studies at the full-text screening stage. See Figure 1 for study flow information.

\section{Included studies}

This review includes 51 RCTs, covering 22,509 participants. Study sample sizes ranged from 24 to 3297. Most trials were conducted in the USA (29 studies); however, trials were also carried out in China (five studies), Germany (two studies), Spain (three studies), Switzerland (three studies), the UK (two studies), Australia, Austria, Canada, Czech Republic, Denmark and New Zealand (one study each). One study (Ebbert 2015) took place across a range of countries (Australia, Canada, the Czech Republic, Egypt, Germany, Japan, Mexico, Taiwan, UK, USA). Four studies randomised participants in clusters rather than individually (Glasgow 1989; Ho 2018; Jerome 1999a; Wu 2017). Further details of how these studies adjusted for clustering and the effects of this are reported in the Characteristics of included studies table.

\section{Participants}

All participants were tobacco smokers, and most (42 of 51 studies) were recruited from the general population, through media or local advertising or both, attendance at primary care or smoking cessation clinics, through their workplace, or through direct mailings and calls from marketing companies. The remaining nine studies recruited people from the following more specific populations:

- People with a sedentary lifestyle (one study; Blevins 2016), i.e. exercising less than 60 minutes a week during the previous six months. This was an inclusion criterion because the intervention involved exercise. Participants were recruited through newspaper and radio advertisements.

- Young people (three studies): Hanson 2008 and Perez-Milena 2012 both recruited 13 to 19 year-old participants directly from high schools; NCT00158158 also recruited 13 to 19 year-olds but did not report how they identified participants.

- Men (two studies): Hao 2017 and Wu 2017 both recruited from outpatient clinics (a smoking cessation clinic and an endocrinology and acupuncture clinic respectively), where all visiting patients were invited to take part. The resulting samples were predominantly male (Hao 2017 97\% male; Wu 2017 100\% male).

- People diagnosed with acute health problems (three studies): Joseph 2008 specifically recruited people diagnosed with cardiovascular diseases through media advertisements and physician referral; Ostroff 2014 recruited hospital outpatients who had recently been diagnosed with cancer and were awaiting surgery; and Rohsenow 2016 recruited participants in a residential treatment programme for substance use disorders.

Across studies, the percentage of women in the sample ranged from $0 \%$ to $85 \%$ (mean $48 \%$ across 46 studies) and average baseline cpd ranged from 11 to 31, with a mean of $23 \mathrm{cpd}$ (across 46 studies). Thirty-two of the 51 included studies reported the Fagerström Test for Nicotine Dependence (FTND) as a measure of baseline nicotine dependence; average values ranged from 3.0 to 7.3 across stud- 
ies, with a mean of 5.8. We extracted data on whether participants preferred reducing smoking to quit or quitting abruptly at study baseline but only two studies measured this. Hughes 2010 found that on a scale of 0 to 10 (where 0 was favouring abrupt quitting and 10 was favouring reduction) the average response was a score of four, suggesting that preference was quite evenly split between the two approaches, with a small preference for quitting abruptly. Lindson-Hawley $2016 \mathrm{~b}$ found that $32.1 \%$ of their sample favoured abrupt quitting, $50.9 \%$ reducing to quit, and that $16.9 \%$ had no particular preference for one method over the other.

\section{Reducing-to-quit interventions}

Reduction methods tested varied greatly across studies: some studies simply asked participants to reduce the amount they smoked (e.g. Caldwell 2016), whereas others provided detailed instructions or suggestions. Some studies straddled these two approaches by providing participants with multiple ideas for ways to reduce, but ultimately allowing them to choose their own approach (e.g. Joseph 2008). In some cases it was difficult to know whether participants were given any guidance on how to reduce because of the way studies were reported (e.g. Chan 2011). Where it was clear, the reduction methods included the following:

- Providing participants with a goal number of cpd to work toward (e.g. Blevins 2016; Cinciripini 1995; Flaxman 1978; Lindson-Hawley 2016b; Perez-Milena 2012). In some cases this was specified as reducing by a set number of cpd (e.g.Blevins 2016). In others, participants were told to reduce by a certain percentage of their baseline rate, such as 50\% (Flaxman 1978). Some studies suggested setting goals but allowed participants to decide the parameters for this themselves (e.g. Carpenter 2004). In some studies, participants were advised to gradually reduce until they were smoking no cigarettes (e.g. Ho 2018; Jerome 1992), whereas in other studies, participants were advised to reduce to a certain amount (e.g. 75\%) and then stop smoking altogether (e.g. Carpenter 2003; Etter 2009). The length of time over which participants were advised to reduce varied greatly between studies, from approximately one week to 18 months.

- Participants were instructed to reduce their consumption by gradually increasing the time between cigarettes (e.g. Cinciripini 1995; Cinciripini 2006; Jerome 1992; Jerome 1999a; Klemperer 2017; Ostroff 2014; Riley 2001). This was typically effected by taking a participant's baseline smoking rate and dividing it by the amount of waking time in the day. This gave participants their baseline inter-cigarette interval $(\mathrm{ICI})$. This $\mathrm{ICI}$ was then gradually increased so that participants' smoking breaks became farther apart. In some studies a handheld computerised device was used to programme in the $\mathrm{ICI}$ (e.g. Jerome 1992; Jerome 1999a; Ostroff 2014; Riley 2001), so that participants could be alerted when it was time for them to smoke. Some studies called this approach scheduled smoking (e.g. Cinciripini 1995; Cinciripini 2006).

- Participants were asked to gradually increase the time in the morning between waking and having their first cigarette (e.g. Wennike 2003).

- Participants were asked to identify routine cigarettes that they smoked at specific times, and then develop a plan for gradually eradicating the individually-identified cigarettes. Some studies called this approach hierarchical reduction, as participants were asked to choose to eliminate either their preferred or least pre- ferred cigarettes first (e.g. Brockway 1977; Hughes 2010; Klemperer 2017; Lindson-Hawley 2016b; Wang 2017).

- Participants were advised not to smoke in particular situations, such as at home or work (e.g. Farley 2017; Flaxman 1978; Joseph 2008; Lindson-Hawley 2016b). Sometimes these situations were suggested and sometimes participants chose the situations themselves. This approach focused less on reducing cpd and more on reducing the amount of time in the day when a person could smoke. This approach was called a smoke-free periods (sfp) approach by some studies.

- Participants were advised to replace cigarettes not smoked with a form of smoking cessation pharmacotherapy. Twenty-six of the 51 included studies provided, or advised participants to use, a form of smoking cessation pharmacotherapy whilst they reduced, before they quit smoking. Eight of these studies provided a choice of individual NRT products or different NRT products were offered in different trial arms, e.g. patches or a form of fast-acting NRT (Carpenter 2003; Carpenter 2004; Chan 2011; Cook 2016; Etter 2002; Farley 2017; Hanson 2008; Joseph 2008). One study advised patch use as well as a form of fast-acting NRT (Lindson-Hawley 2016b), three offered nicotine patch only (Cinciripini 2006; NCT00158158; Rohsenow 2016), 11 offered a form of fast-acting NRT only, such as gum, lozenge or nasal spray (Bolliger 2000a; Caldwell 2016; Dooley 1992; Etter 2009; Haustein 2002; Hughes 2010; Kralikova 2009; Rennard 2006; Riley 2005; Shiffman 2009; Wennike 2003), two advised varenicline use (Ebbert 2015; Hao 2017) and one bupropion (Hatsukami 2004). It was unclear from the trial report of one study whether pharmacotherapy was offered during reduction or only after quitting had occurred (Ostroff 2014). None of the included studies advised participants to replace tobacco cigarettes with an electronic cigarette.

Twenty-nine studies provided participants in at least one reduction arm with a very specific quit date to work toward, which was either clear from the outset or implied by the nature of the reduction schedule advised (e.g. Etter 2009; Ho 2018; Lindson-Hawley 2016b; Perez-Milena 2012; Riley 2005), or encouraged participants to set their own quit dates to work towards (e.g. Carpenter 2003; Hanson 2008; Rohsenow 2016). However, others did not appear to suggest a time point to work toward at all (e.g. Bolliger 2000a; Etter 2002). Although all of the studies in this review had to provide some encouragement or advice to quit in order to be eligible for inclusion, in some studies this was not framed as the main aim of the intervention; participants were advised to try and reduce their smoking and were provided with an ongoing choice of whether they would like to progress to cessation (e.g. Glasgow 1989; Malott 1984).

Many studies provided participants with additional smoking-cessation treatment components alongside the reduction advice provided, for example, information about the dangers of smoking, smoking cessation pharmacotherapy, or relapse prevention counselling. A small number of studies we identified combined advice to reduce smoking behaviour with advice or the means to carry out nicotine fading (e.g. Garcia 2000; Glasgow 1989; Nicki 1984). This is where people gradually switch to cigarette brands that advertise lower nicotine yield. We decided to include this subset of studies, as the fact that participants were also asked to reduce their smoking meant they met our inclusion criteria. However, we excluded studies that asked participants to carry out nicotine fading without any advice to reduce the amount they were smoking. 


\section{Intervention modality and intensity}

In most cases ( 38 of the 51 studies) at least some of the intervention support in the reduction arms was provided face-to-face; in some cases this was also augmented by telephone calls, text messages, self-help materials and computerised support to set and meet reduction targets. In four studies support was offered by telephone (Carpenter 2004; Glasgow 2009a; Hughes 2010; Klemperer 2017), and in a further five studies only self-help materials were provided (Cummings 1988; Etter 2002; Etter 2009; Jerome 1999a; Shiffman 2009). Self-help alone was most commonly offered in the form of printed materials, but one of the reduction arms in Jerome 1992 received a handheld computer designed to implement ICls alongside a printed booklet. The modality of the support provided in four of the studies was unclear (Cinciripini 2006; Haustein 2002; NCT00158158; Riley 2001).

The overall contact time and number of sessions provided for reduction interventions providing some kind of person-to-person contact (i.e. not self-help only) varied greatly from six minutes to 16 hours of contact, delivered over one to 28 sessions.

\section{Comparators}

There were three comparator interventions eligible for inclusion in this review: 1) no smoking cessation intervention; 2) abrupt quitting interventions; 3 ) another smoking reduction intervention.

\section{No smoking cessation intervention}

Six studies compared a smoking reduction-to-quit intervention with no smoking cessation treatment (Brockway 1977; Carpenter 2004; Cook 2016; Glasgow 2009a; Ruther 2018; Wu 2017). Brockway 1977, Carpenter 2004 and Cook 2016 all provided no treatment in the relevant comparator arm, with participants only contacted for follow-ups to collect data. Ruther 2018 was a waitlist control, so comparator participants did not receive treatment during the study, but were offered the smoking reduction intervention after follow-up was complete. Glasgow 2009a and Wu 2017 both offered comparator participants an alternative intervention, which did not focus on smoking cessation. Participants in Glasgow 2009a received three quarterly healthcare education mailings that did not specifically focus on smoking, and Wu 2017 provided participants with exercise and diet advice. This latter intervention was designed to match the intensity of the smoking reduction intervention; in both study arms the intervention was delivered in a total of six minutes and involved six contacts with investigators. However, the reduction intervention in Glasgow 2009a was more intensive than the health mailing comparator, as it involved four phone calls, as well as a newsletter.

\section{Abrupt quitting interventions}

Twenty-seven studies compared a smoking reduction-to-quit intervention with a smoking cessation intervention that did not advise participants to reduce the amount they were smoking before quitting altogether. For the purposes of this review we describe these studies as 'abrupt quitting' interventions. The studies themselves did not always define the intervention in this way. In fact, in many instances (as in the case of the reduction interventions, and more widely across smoking cessation studies) it was difficult to identify the full content of the interventions because they were reported in insufficient detail. In some trials, participants in the abrupt-quitting condition spontaneously reduced cpd before their quit date. However, if a person was not advised to reduce their smoking be- fore quitting by investigators then any decision to do so was made by participants and so we did not class this as a reduction intervention. The abrupt-quitting intervention varied between studies and was offered face-to-face, over the phone, through self-help materials, or through a combination of these approaches. In some studies the participants in the abrupt group were asked to quit immediately (e.g. Flaxman 1978; Hughes 2010), whereas in others they received some preparatory treatment before being asked to quit altogether (e.g. Lindson-Hawley 2016b). Overall contact time for the abruptquitting comparator ranged from one minute to 16 hours and was delivered in between one and 12 sessions. Nineteen of these studies included at least one abrupt-quitting arm where the intensity of the support provided was matched to the reduction-to-quit intervention (three included two abrupt arms, i.e. one that was very brief plus a more intensive intervention; Flaxman 1978; Hughes 2010; Klemperer 2017); five had a reduction arm that was more intensive (Carpenter 2003; Chan 2011; Cook 2016; Joseph 2008; Perez-Milena 2012), and the relative intensity of interventions was unclear in the remaining three studies (Cinciripini 2006; NCT00158158; Riley 2001).

In 14 studies, smoking cessation pharmacotherapy was used prequit in the reduction-to-quit arm and was also given to people in the abrupt arm, but only after the quit day. However, there were some exceptions: participants in the abrupt arm in Chan 2011, Dooley 1992 and Joseph 2008 did not use pharmacotherapy despite the reduction arm receiving NRT, and participants in the abrupt arms in Cook 2016, Lindson-Hawley 2016b and NCT00158158 used NRT pre- and post-quit.

Two of these studies included nicotine fading advice in the abruptquitting arm, i.e. advice to switch cigarette brands to those advertising less nicotine yield (Dooley 1992; Nicki 1984). These were deemed eligible for inclusion in this subset of studies, as participants were not asked to reduce their smoking behaviour, but we analysed them separately from studies where nicotine fading was not a main component of the abrupt quitting arm.

\section{Other smoking reduction interventions}

Twenty-nine of the 51 included studies compared the effectiveness of two or more methods to assist reduction to quit. Twelve of these studies aimed to investigate whether using pharmacotherapy to aid smoking reduction was more effective than using no pharmacotherapy or placebo; 10 used NRT (either patch alone, fast-acting NRT alone, or combination NRT; Bolliger 2000a; Caldwell 2016; Cook 2016; Etter 2009; Hanson 2008; Haustein 2002; Kralikova 2009; Rennard 2006; Shiffman 2009; Wennike 2003), and one each tested varenicline (Ebbert 2015) and bupropion (Hatsukami 2004). Most studies contributing to this comparison used a matched placebo in the comparator arm, but Cook 2016 compared NRT treatment to no pharmacotherapy and Hanson 2008 compared NRT to a nonmatched placebo (folic acid pills). All other intervention content was matched between trial arms.

A number of additional comparisons were tested by the studies with more than one reduction-to-quit intervention arm; however substantially fewer studies contributed to each comparison, as detailed below:

- Modality of support provided (five studies): Curry 1988, Farley 2017 and Garcia 2000 all compared a reduction intervention delivered using behavioural support with the same reduction pro- 
gramme delivered using self-help resources. In all cases this was a booklet or manual. Curry 1988 and Garcia 2000 both specified that the manuals included exercises for participants. Glasgow 1978 and Jerome 1992 compared a self-help reduction programme, delivered as a booklet and a handheld computer respectively, to the same self-help programme, plus additional behavioural support.

- Length of smoking reduction period (two studies): Farley 2017 compared a reduction programme where participants were advised to reduce over four weeks and then quit versus a programme where participants were asked to reduce over 16 weeks and then quit. Haustein 2002 compared a programme where participants were asked to reduce and then quit over four weeks versus a programme where they were asked to reduce over nine months in total, with prompts to quit at six months and nine months.

- More versus less structured reduction methods (two studies): Cinciripini 1995 had a reduction-to-quit group who reduced their smoking by gradually increasing the time between cigarettes (their $\mathrm{ICl}$ ) over three weeks, reducing by a third of their baseline consumption each week. Participants could smoke only within the first five minutes of each ICl. This was compared with a second reduction group who were asked to gradually reduce their consumption by the same amount as the first group, but ICls were not calculated and participants could smoke their cigarette quotas whenever they wanted. Cummings 1988 randomised participants to two reduction groups, one of which received a high-structure booklet and another which received a low-structure booklet. The booklets were the same length and both instructed smokers to gradually reduce the number of cigarettes smoked over a brief period before quitting altogether. Participants were given a number of suggestions about how to reduce, such as setting daily goals, switching brands, changing habits, and delaying the first cigarette of the day. Participants in the high-structure group were asked to read the booklet every day and carry out the activities for that day, whereas the lowstructure group did not receive the information in the same daily structure and were asked to examine the menu of information in the booklet and select the exercises they felt would be helpful rather than working through them systematically.

- Additional behavioural smoking cessation support or components (four studies): all four studies compared identical reduction programmes, with one group receiving a form of additional behavioural support. Chan 2011 added NRT adherence counselling to their existing smoking reduction plus nicotine patches intervention. Garcia 2000 delivered the same smoking reduction programme either in five sessions over five hours or in 10 sessions over 10 hours. In Malott 1984 two groups of participants took part in group behavioural support sessions at their worksite, focused on smoking reduction. In one of the groups participants were also paired with a partner (a co-worker) with whom they could discuss progress on a daily basis. Nicki 1984 advised two study groups to carry out a reduction programme where they gradually stopped smoking in particular situations. In one of these groups participants were also provided with "self-instructional training". Participants were told that the way they talked to themselves or did not talk to themselves may have an effect on their smoking behaviour. Examples were given of appropriate self-instructions that could be implemented before, during and after a smoking situation. Participants were asked to develop patterns of thought that could be applied to their own smoking situations.

Finally, five studies compared individual interventions that could not be grouped with any other study. Blevins 2016 compared a general health intervention to an aerobic exercise intervention. During both interventions participants were instructed to practice the reduction of one to two cigarettes a day prior to quitting altogether. Flaxman 1978 compared a reduction programme where participants were asked to reduce the amount they smoked to zero with a programme where participants were asked to reduce to $50 \%$ of baseline before then quitting abruptly. Gariti 2004 provided two groups with a smoking-reduction intervention, one of which was supplemented by a nicotine-fading intervention. In one study group Glasgow 1989 assisted participants to select strategies to reduce the number of cigarettes they smoked per day to between $50 \%$ and $67 \%$ of baseline, then between $33 \%$ and $50 \%$ of baseline. Participants were then given the choice of quitting (recommended option) or of making further changes to smoking topography, whilst continuing to smoke at reduced levels. In the second group a quit date was explicitly stated and participants targeted individual cigarettes to eliminate, although specific reduction goals were not provided. Rohsenow 2016 investigated whether a smoking cessation intervention was more effective when participants were provided with vouchers contingent on reduced exhaled CO readings or non-contingent on reduced $\mathrm{CO}$ readings.

\section{Outcomes}

In order to be eligible for this review, studies had to measure smoking cessation rates at least six months after baseline. However, five of the included studies did not go on to report these rates (Cinciripini 2006; NCT00158158; Riley 2001), or did not report them by trial arm (Glasgow 1978; Hanson 2008). We contacted the authors of these studies, but we either received no response or the authors were unable to supply the data we needed. We were therefore unable to include them in our analyses. Of the remaining 46 studies that did report abstinence rates by arm, most reported point prevalence (pp) abstinence ( 27 studies), 10 reported prolonged abstinence and six reported continuous abstinence. In three studies the definition of abstinence was unclear (Flaxman 1978; Joseph 2008; Nicki 1984). The most common follow-up endpoints were six months (19 studies) and 12 months ( 21 studies). Five further studies used endpoints of 15 months, 18 months or 24 months, with one study measuring follow-up five years post-baseline (Etter 2002).

Twelve studies reported on the number of quit attempts made in each group. In most cases this was defined as at least 24 hours of smoking abstinence (nine studies); however the following definitions were also used in one study each: 1 ) at least one serious attempt since receiving the intervention (Cummings 1988); 2) abstinent three days after the quit day (Etter 2009); 3) abstinent at the end of the reduction phase (Gil Roales-Nieto 1992a).

Twenty-four studies reported data on the reduction in cigarette consumption before quitting that we could use in our analyses. Other studies reported on reduction in general, but this was not always split into a pre-quit and a post-quit period. In some cases reduction was measured pre-quit in the reduction arm, but if the comparison was an abrupt quitting intervention, in which participants were immediately asked to quit, it was impossible to compare pre-quit smoking consumption between the two arms. Where there was not a clear quit date specified, it was hard to define the 
pre-quit period; in these cases we took the measurement of reduction closest to the end of any recommended reduction programme. As anticipated in our protocol, some studies reported reduction dichotomously (e.g. Ebbert 2015; Lindson-Hawley 2016b; Ruther 2018), by reporting how many people had met a prespecified reduction threshold ( $50 \%$ in most cases and $75 \%$ in one case), and some studies reported it continuously (e.g. Cinciripini 1995; Etter 2002; Garcia 2000). Most studies measured cpd as the marker of reduction (e.g. Hughes 2010; Klemperer 2017; Wu 2017), but some studies also measured CO (Bolliger 2000a; Glasgow 1989; Hanson 2008; Lindson-Hawley 2016b) and cotinine (Cinciripini 1995; Gariti 2004).

Eleven studies provided data on the number of participants who reported AEs or SAEs during the pre-quit period, and seven studies reported on pre-quit withdrawal symptoms (Caldwell 2016; Cinciripini 1995; Etter 2009; Haustein 2002; Hughes 2010; Gariti 2004; Glasgow 1989).
Three studies had useable data on the potential effects of baseline moderators (self-efficacy, motivation to quit, preference for reduction over abrupt quitting) from three studies (Curry 1988; Hughes 2010; Lindson-Hawley 2016b). All three compared reduction to quit with abrupt quitting. We summarise this outcome narratively.

\section{Excluded studies}

We listed 196 studies that were potentially relevant but excluded, with reasons, in the Characteristics of excluded studies table. Reasons for exclusion at full-text stage are also summarised in Figure 1. The most common reasons studies were excluded at full-text screening stage were because they were not testing a behavioural smoking reduction-to-quit intervention, or because they did not follow up at six months or longer from baseline.

Figure 1. Study flow diagram.

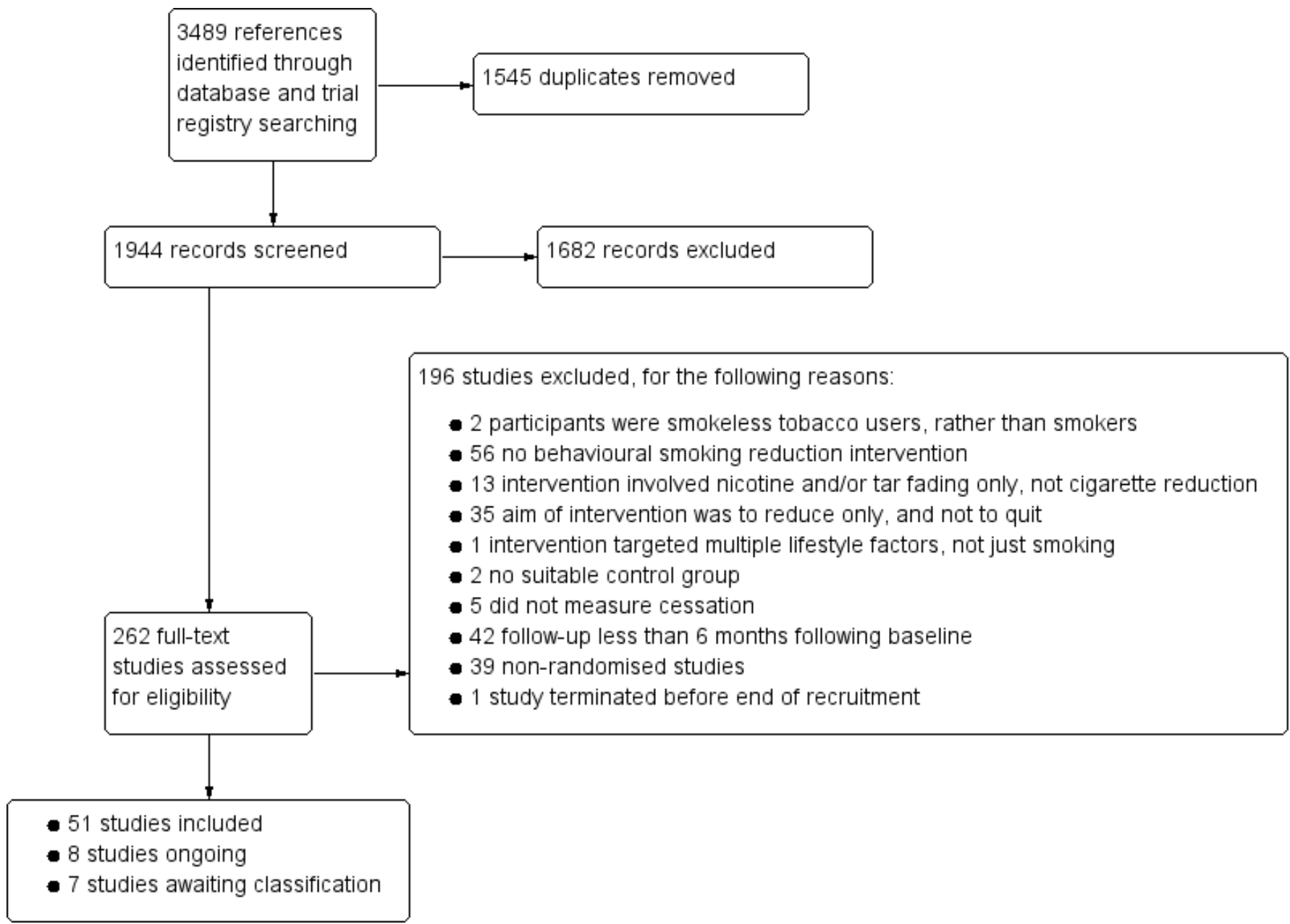

We categorised seven studies as 'awaiting classification' (Cinciripini 2001; Cooper 1990; Engeln 1969; Gardner 1971; Palmer 1983; Rennard 1994; Weis 1974). For all of these studies only a title was available and it was impossible to be sure whether they met our inclusion criteria. Where we were able to locate contact details we contacted authors for further information, but there was either no response or the author was unable to provide the required informa- tion (see the Studies awaiting classification table for further information).

\section{Risk of bias in included studies}

Full details of 'Risk of bias' assessments are given for each trial within the Characteristics of included studies tables. Overall, we judged five studies to be at low risk of bias (low risk of bias across all do- 
main), and the remaining 28 at unclear risk of bias. A summary illustration of the 'Risk of bias' profile across trials is shown in Figure 2. 
Figure 2. Risk of bias summary: review authors' judgements about each risk of bias item for each included study.

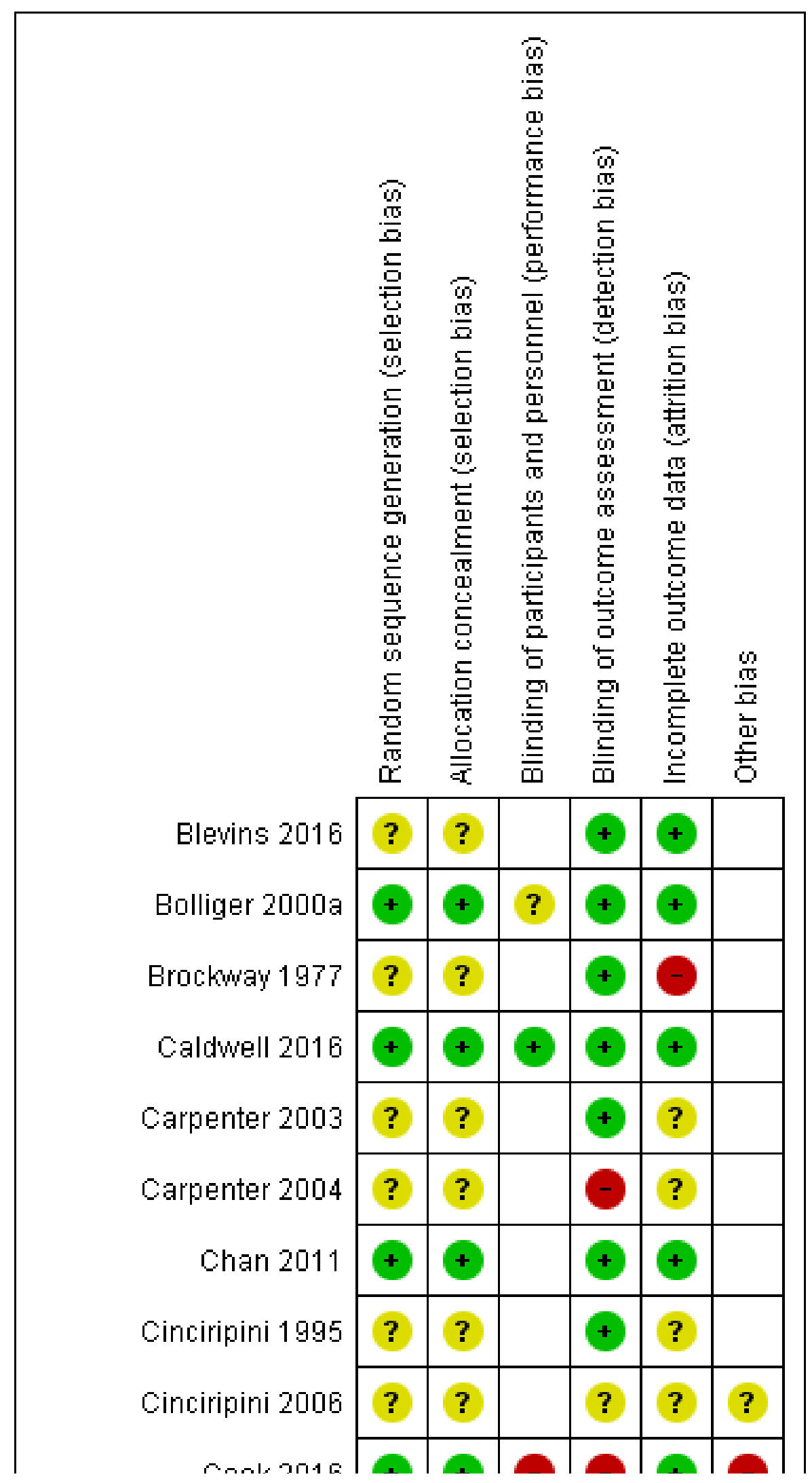


Figure 2. (Continued)

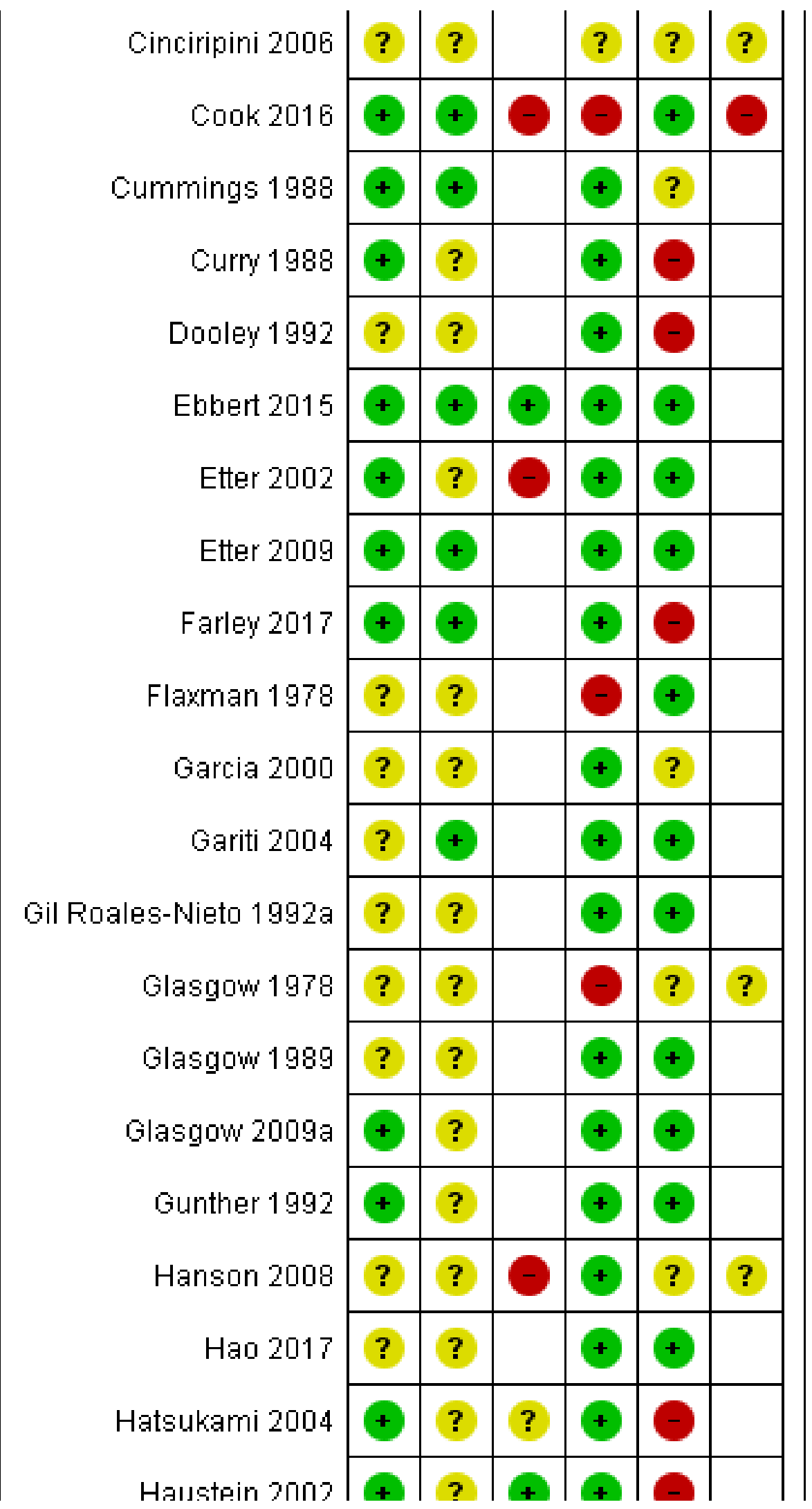


Figure 2. (Continued)

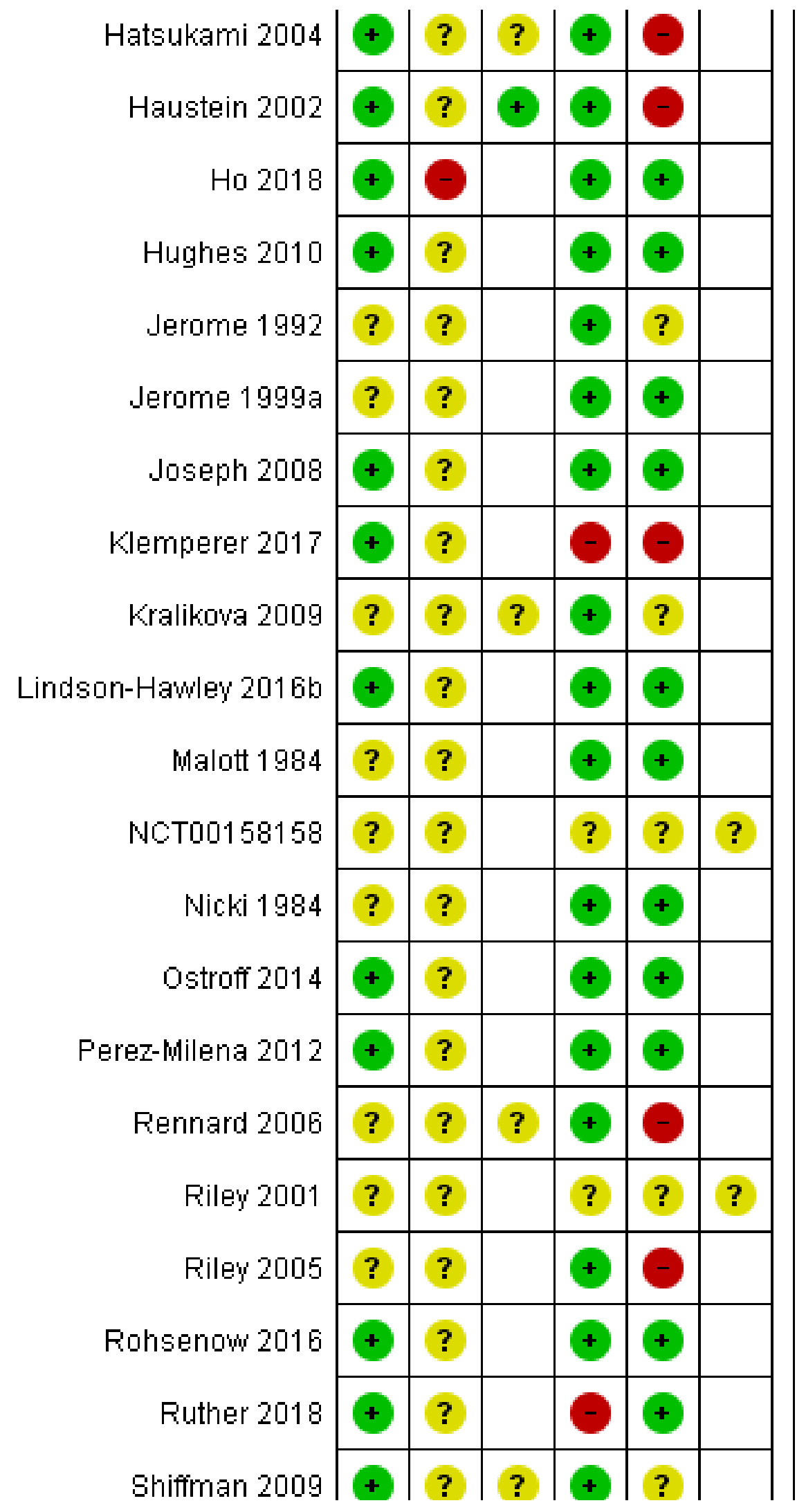


Figure 2. (Continued)

\begin{tabular}{|c|c|c|c|c|c|}
\hline Ruther 2018 & + & $?$ & & & \\
\hline Shiffman 2009 & + & $?$ & $?$ & + & $?$ \\
\hline Wang 2017 & $?$ & $?$ & & & \\
\hline Wennike 2003 & $?$ & $?$ & $?$ & & \\
\hline Wu 2017 & + & + & & + & \\
\hline
\end{tabular}




\section{Allocation}

We assessed selection bias through investigating methods of random sequence generation and allocation concealment for each study. We rated 25 studies as being at low risk for random sequence generation, and the remaining 26 as having unclear risk. We judged 10 studies to be at low risk for allocation concealment, 40 at unclear risk, and one study at high risk (Ho 2018). We rated Ho 2018 at high risk, as participants were allocated to intervention groups by week of enrolment and not individually. This suggests that researchers knew which treatment each week would be allocated when participants attended for enrolment. We judged studies at unclear risk of bias when authors provided insufficient information about the methods used.

\section{Blinding}

We assessed performance bias only for those trials where the use of pharmacotherapy to assist smoking reduction was being investigated. It is almost always impossible to blind providers of behavioural support to treatment allocation. Moreover, nonspecific effects of being in treatment were part of the intervention effect that studies in this review were aiming to assess. We therefore assessed only detection bias for all studies that were not specifically testing the effects of pharmacotherapy.

Twelve studies compared a reduction method aided by smoking cessation medication with a reduction method implemented alongside a placebo medication or no medication. We judged three of the 12 to be at low risk of performance bias, three at high risk and the remaining six at unclear risk. Studies were judged to be at high risk for the following reasons: Cook 2016 compared NRT to no smoking cessation medication rather than a placebo, mak ing blinding impossible; Etter 2002 reported that study investigators were not blind to treatment allocation despite a placebo being used; and Hanson 2008 randomised control participants to folic acid tablets rather than to a placebo matched to NRT, and stated that the study was not double-blinded. We rated studies at unclear risk of bias when a matched placebo was used and a study claimed to be double-blinded, but the authors did not specify exactly who was blinded. This is in line with standard Cochrane guidance (Higgins 2017).

We judged detection bias on the basis of biochemical validation and, where biochemical validation was not used, on the basis of different levels of contact between participants and the study team across relevant study groups. We judged six studies to be at high risk of detection bias, as outcomes were defined as self-report only and the intervention and control arms received different levels of support, making differential misreporting possible (Carpenter 2004; Cook 2016; Flaxman 1978; Glasgow 1978; Klemperer 2017; Ruther 2018). Additionally Ruther 2018 used a waitlist control design, meaning that people in this arm may have been less likely to try and quit and may have been waiting to receive treatment to do so. We judged three studies to be at unclear risk of detection bias (Cinciripini 2006; NCT00158158; Riley 2001), as we were unsure whether abstinence was biochemically verified. We judged the remaining 42 studies to be at low risk of detection bias.

\section{Incomplete outcome data}

We judged studies to be at a low risk of attrition bias where the numbers of participants lost to follow-up were clearly reported, the overall number lost to follow-up was not more than $50 \%$, and the difference in loss to follow-up between groups was no greater than $20 \%$. This is in accordance with 'Risk of bias' guidance produced by the Cochrane Tobacco Addiction Group for assessing smoking cessation studies. We judged 28 of the studies to be at low risk of bias, 13 at unclear risk and 10 at high risk. We rated eight studies (Brockway 1977; Farley 2017; Hatsukami 2004; Haustein 2002; Klemperer 2017; Rennard 2006; Riley 2005; Wennike 2003) at high risk because overall loss to follow-up was more than $50 \%$, whereas Curry 1988 and Dooley 1992 were judged at high risk due to different dropout rates between groups (greater than $20 \%$ difference). We made judgements of unclear risk either because information on follow-up at the relevant time point was not reported overall, or because it was not reported split by study groups.

\section{Other potential sources of bias}

We identified two additional potential sources of bias for six of the included studies. Cook 2016 was a factorial trial with four factors: 1) motivational interviewing (MI)/no MI; 2) behavioural reduction counselling/no behavioural reduction counselling; 3) nicotine gum/no nicotine gum; and 4) nicotine patch/no nicotine patch. The authors reported an unexpected interaction between MI (the abrupt-quit group for the purposes of this review) and nicotine gum, where the combination of the two resulted in lower quit rates than any other interventions or combinations. As a result, we have assigned Cook 2016 a rating of high risk of other bias. For details of how data from Cook 2016 have been entered into meta-analyses, see the Characteristics of included studies table. We judged five studies (Cinciripini 2006; Glasgow 1978; Hanson 2008; NCT00158158; Riley 2001) to be at unclear risk of other bias for the same reason; they all reported that they measured (or planned to measure) abstinence at long-term follow-up (six months or more), but long-term abstinence has not yet been reported, or has been reported overall, but not split by study group. In all these cases we attempted to contact the authors, but they either did not reply or were unable to provide the data due to the length of time since the end of the study. We can not be sure whether or not any lack of reporting was due to actual bias (i.e. selective reporting).

\section{Effects of interventions}

See: Summary of findings for the main comparison Reduction to quit versus abrupt quitting for smoking cessation; Summary of findings 2 Reduction to quit versus no treatment for smoking cessation; Summary of findings 3 Reduction and pharmacotherapy versus reduction alone for smoking cessation

We report outcomes by comparison. Where an outcome is not reported for a comparison this is because no studies within that comparison reported useable data.

\section{'Reduction to quit versus no treatment' comparison}

\section{Abstinence}

We pooled six studies with 1599 participants relevant to this comparison, resulting in moderate heterogeneity $(12=45 \%)$. Although the point estimate favoured reduction-to-quit treatment over no smoking cessation treatment there was substantial imprecision, meaning that the result could indicate potential minimal harm as well as considerable benefit (RR $1.74,95 \% \mathrm{Cl} 0.90$ to 3.38; Analysis 1.1). Removing those studies judged to be at high risk of bias left only two studies in the analysis (Glasgow 2009a; Wu 2017), but the 
interpretation of the result remained the same (RR $1.96,95 \% \mathrm{Cl} 0.96$ to $4.00 ; 1^{2}=45 \% ; 2$ studies, 758 participants).

We also carried out the main analysis split by the type of pharmacotherapy used to aid reduction to quit. Two trials were included in the nicotine patch, nicotine gum or combination NRT subgroup $(\mathrm{N}=633$ ) and five in the no-pharmacotherapy subgroup ( $\mathrm{N}=966)$, with study arms from Cook 2016 appearing in both groups (Analysis 1.2). There was no evidence of any difference between subgroups $\left(I^{2}=0 \%, P=1.00\right)$.

\section{Quit attempts}

Only one study (419 participants) reported data relevant to this outcome. Carpenter 2004 found a benefit of reduction-to-quit interventions on the number of quit attempts made when compared with no smoking cessation treatment (RR $2.78,95 \% \mathrm{Cl} 1.95$ to 3.96; $\mathrm{I}^{2}=\mathrm{n} / \mathrm{a}$; Analysis 1.3).

\section{Pre-quit reduction}

Two studies provided adequate data for this outcome (Ruther 2018; Wu 2017), with a total of 473 participants (Analysis 1.4). Both of these studies assessed reduction in cpd as a binary measure with $50 \%$ as the cut-off point. The pooled data provided evidence that more people reduced their smoking by at least $50 \%$ of their baseline consumption in the reduction-to-quit groups than the no smoking cessation treatment groups ( $\mathrm{RR} 1.79,95 \% \mathrm{Cl} 1.28$ to $2.51 ; \mathrm{I}^{2}=0 \%$ ).

\section{Pre-quit SAES}

Only one study covered the number of SAEs reported in the pre-quit period (Cook 2016; 291 participants). No SAEs were reported in any of the study arms.

\section{'Reduction to quit versus abrupt quitting' comparison}

\section{Abstinence}

We identified 22 studies that investigated this comparison and reported data adequately for use in our analyses. There was no evidence of any substantial difference in long-term abstinence rates between those advised to reduce their smoking before quitting and those advised to quit abruptly, with a point estimate very close to the null (RR 1.01, 95\% Cl 0.87 to $1.17 ;\left.\right|^{2}=29 \%$; 22 studies, 9219 participants; Analysis 2.1). Removing seven studies judged to be at high risk of bias resulted in a very similar effect estimate (RR 1.05, 95\% $\mathrm{Cl} 0.88$ to $1.25 ; \mathrm{I}^{2}=38 \%$; 15 studies, 7037 participants). We also carried out a sensitivity analysis removing comparisons where the reduction intervention was more intensive than the abrupt intervention, to try and reduce any effect that more intensive support could have had on quit rates. Seventeen studies remained in the analysis (Analysis 2.2), with a total of 6656 participants. There was only a minimal impact on the point estimate, with Cls still spanning both benefit and harm of reduction to quit in comparison to abrupt quitting (RR $0.95,95 \% \mathrm{Cl} 0.80$ to $1.12 ; 1^{2}=40 \%$ ). Similarly, we carried out an analysis removing two studies where pharmacotherapy was provided to participants in the reduction-to-quit arms but participants in the abrupt arms did not receive pharmacotherapy at any point (Chan 2011; Joseph 2008). Again this made minimal difference to the pooled result ( $\mathrm{RR} 0.99,95 \% \mathrm{Cl} 0.85$ to $1.15 ; \mathrm{I}^{2}=29 \%$; 20 studies, 7913 participants).

As there were sufficient data included in this analysis, we carried out a number of prespecified subgroup analyses to explore whether any characteristics of reduction-to-quit interventions modified their effect on smoking cessation, relative to abrupt quitting. We first split the studies according to the type of pharmacotherapy used pre-quit in the reduction-to-quit groups (Analysis 2.3): varenicline (1 study, 314 participants); NRT (9 studies, 4359 participants); no pharmacotherapy (13 studies, 4546 participants). This resulted in a significant subgroup difference $(12=77 \%, P=0.01)$, driven by the varenicline subgroup, which found a statistically and clinically significant benefit of reduction to quit on smoking cessation when compared with an abrupt-quitting intervention (RR $1.48,95 \% \mathrm{Cl}$ 1.16 to $1.90 ; 1^{2}=n / a ; 314$ participants), whereas both the NRT (RR $0.91,95 \% \mathrm{Cl} 0.72$ to $1.16 ; \mathrm{I}^{2}=26 \%$; 4359 participants) and no-pharmacotherapy groups (RR $1.01,95 \% \mathrm{Cl} 0.85$ to $1.19 ;\left.\right|^{2}=0 \%$; 4546 participants) found no evidence of superiority for the abrupt or reduction-to-quit interventions.

We also carried out the following subgroup analyses:

- Grouping by whether the study set a specific quit date for participants (Analysis 2.4): 1) quit date set (14 studies); 2) no quit date set (6 studies); 3 ) unclear whether a quit date was set (2 studies).

- Grouping by whether reduction methods focused on reducing cigarettes per day (cpd) or the number of smoke-free periods (sfps) in a day (Analysis 2.5): 1) cpd (14 studies); 2) sfp (1 study); 3) choice of cpd or sfp (6 studies); 4) unclear which methods were recommended (1 study).

- Grouping by whether more structured advice on how to reduce by a specified amount were provided or whether advice on reduction was less specific and unstructured (Analysis 2.6): 1) structured reduction advice (16 studies); 2 ) unstructured reduction advice ( 6 studies); 3) unclear how structured reduction advice was (1 study).

- Grouping by the length of the smoking reduction period (Analysis 2.7): 1 ) $\leq 4$ weeks (13 studies); 2 ) 5 to 13 weeks ( 5 studies); 3 ) 6 months (1 study); 4) 18 months (1 study); 5) unclear (2 studies).

- Grouping by the smoking reduction goals set by investigators (Analysis 2.8): 1) reduce by $<50 \%$ of baseline consumption (1 study); 2 ) reduce by $50 \%$ of baseline consumption ( 4 studies); 3 ) reduce by $75 \%$ to $85 \%$ of baseline consumption ( 3 studies); 4 ) reduce by $100 \%$ of baseline consumption ( 7 studies); 5 ) reduction goals chosen by participants ( 5 studies); 6 ) no goals stated (3 studies).

None of the listed analyses showed any evidence of moderating the effect of reduction-to-quit interventions in comparison with abrupt quitting $\left(\mathrm{I}^{2}=0 \% ; \mathrm{P}>0.05\right.$ in all cases).

\section{Quit attempts}

Eleven studies provided data for this outcome (5389 participants). Our pooled analysis found evidence that a smaller proportion of people in the reduction-to-quit arms made a quit attempt than the abrupt-quit participants (RR $0.92,95 \% \mathrm{Cl} 0.85$ to 0.99 ; Analysis 2.9), although we found moderate heterogeneity $\left(1^{2}=46 \%\right)$.

\section{Pre-quit reduction}

One study (697 participants) assessed the proportions of participants who reduced consumption by more than $50 \%$ or not (Lindson-Hawley 2016b). Assessed by both cpd (RR 3.21, 95\% Cl 2.44 to 4.23; $\left.\mathrm{I}^{2}=\mathrm{n} / \mathrm{a}\right)$. and exhaled $\mathrm{CO}(\mathrm{RR} 2.80,95 \% \mathrm{Cl} 2.09$ to $3.75 ; \mathrm{I}=\mathrm{n} / \mathrm{a})$, halving consumption pre-quit was much more likely in the reduc- 
tion than in the abrupt arm (Analysis 2.10). Five studies reported on reduction in cigarette consumption and presented these measures continuously (Cinciripini 1995; Etter 2009; Hughes 2010; Klemperer 2017; Lindson-Hawley 2016b). When we pooled them, there was substantial heterogeneity $\left(1^{2}=99 \%\right)$, and we therefore deem it inappropriate to present a pooled estimate. Point estimates for individual studies all suggested that consumption declined more in the reduction than in the abrupt quit arms (Analysis 2.11); in the case of one study (two comparisons) the Cls spanned zero (Cinciripini 1995). Two studies measured pre-quit reduction using a continuous measure of CO (Lindson-Hawley 2016b) or a continuous measure of cotinine (Cinciripini 1995). Both found evidence that cigarette consumption declined more in the reduction than in the abrupt group (CO: MD 8.10, 95\% Cl 6.56 to 9.64; $\mathrm{I}^{2}=\mathrm{n} / \mathrm{a}$; 697 participants; Analysis 2.12; and cotinine: MD 95.12, $95 \% \mathrm{Cl} 6.60$ to $183.64 ;\left.\right|^{2}=51 \% ; 128$ participants; Analysis 2.13), although the effect estimate for the cotinine analysis was very imprecise.

\section{Pre-quit adverse effects}

Six studies reported on the number of SAEs reported during the prequit period, with a total of 2309 participants (Analysis 2.14). We split these into studies where participants in the reduction arm received pre-quit NRT (N = 1559; Cook 2016; Etter 2009; Joseph 2008; Lindson-Hawley 2016b) and studies where participants did not receive pre-quit pharmacotherapy ( $N=750$; Cook 2016; Ho 2018; Klemperer 2017). In four of the six studies no SAEs were reported in either arm. The two studies that did report pre-quit SAEs were both in the NRT subgroup; neither found evidence of an excess of SAEs in either group (NRT subgroup pooled estimate: RR $1.19,95 \% \mathrm{Cl} 0.63$ to 2.27 ; $12=0 \% ; 1559$ participants).

We were able to extract some additional narrative information on adverse effects from Lindson-Hawley 2016b, who measured symptoms of nicotine overdose during the pre-quit period. In this study both groups used a nicotine patch pre-quit, but participants in the reduction group also used a form of fast-acting NRT to replace the cigarettes they were not smoking. The study found that most symptoms of nicotine overdose were uncommon and mild, and that rates did not differ between groups; however, there were slightly higher rates of salivating $(18 / 120(15 \%)$ reduction versus $17 / 259(7 \%)$ abrupt), and cold sweats (15/121 (12\%) reduction versus $11 / 261$ (4\%) abrupt) in the reduction group (Analysis 2.15).

Three studies reported on nicotine withdrawal during the pre-quit period (Cinciripini 1995; Etter 2009; Hughes 2010). Further details can be found in Analysis 2.15. Cinciripini 1995 and Etter 2009 both found that some withdrawal symptoms increased during the prequit period but that these changes did not differ between groups, and Etter 2009 and Hughes 2010 both found some reductions in craving. However, in Etter 2009 cravings reduced in both groups, whereas in Hughes 2010 craving only reduced in the reduction condition, whilst staying the same in the abrupt condition. This resulted in significant between-group differences $(P<0.001)$.

\section{Moderation of the effect}

We prespecified that we would report narratively any within-study analyses that had been carried out to investigate baseline motivation to quit, self-efficacy or preference for reduction to quit versus abrupt quitting, as moderators of the effect of reduction-to-quit interventions. Three of the studies within this comparison reported such analyses. These are summarised in Analysis 2.16. Hughes 2010 found that self-efficacy did moderate the effect of the intervention, with abrupt quitting more effective in participants with high self-efficacy, but it did not out-perform reduction in participants with low self-efficacy. Curry 1988 found no evidence that self-efficacy was a moderator. Hughes 2010 did not find statistically significant evidence for an effect of motivation to quit as a moderator, and neither Hughes 2010 and Lindson-Hawley 2016b found evidence that preference for one quitting approach over another moderated the effect of reduction on quitting.

\section{'Reduction with pharmacotherapy versus reduction alone' comparison}

\section{Abstinence}

We identified 11 studies that investigated this comparison and reported data adequately for use in analyses, with a total of 8636 participants. The overall effect estimate for this analysis provided evidence that quit rates were higher in participants assigned to use smoking cessation pharmacotherapy to aid their reduction, when compared with participants assigned to reduce to quit without the help of pre-quit pharmacotherapy (RR $1.68,95 \% \mathrm{Cl} 1.09$ to 2.58 ; $\left.\right|^{2}$ $=78 \%$; Analysis 3.1). A sensitivity analysis removing five studies at high risk of bias increased the estimate of the effect substantially (RR $2.63,95 \% \mathrm{Cl} 1.78$ to $3.89 ; \mathrm{I}^{2}=62 \%$; 6 studies, 6029 participants).

Some but not all of the substantial heterogeneity observed in analysis Analysis 3.1 was explained by the prespecified subgroup analysis that split studies by the type of pharmacotherapy used $(12$ $=80.1 \%, \mathrm{P}=0.007)$. Studies in the combination NRT (RR $1.02,95 \% \mathrm{Cl}$ 0.61 to $1.69 ;\left.\right|^{2}=44 \%$; 3 studies, 1124 participants), nicotine patch (RR $0.34,95 \% \mathrm{Cl} 0.02$ to $5.31 ; \mathrm{I}^{2}=57 \%$; 1 study split into two parts - with and without motivational interviewing, 85 participants) and bupropion (RR $1.27,95 \% \mathrm{Cl} 0.67$ to 2.40 ; $\mathrm{I}^{2}=\mathrm{n}$ /a; 1 study, 594 participants) subgroups found no clear evidence of benefit or harm as a result of using pharmacotherapy; however, the fast-acting NRT (RR 2.56, $95 \% \mathrm{Cl} 1.93$ to $3.39 ; \mathrm{I}^{2}=0 \% ; 7$ studies, 5323 participants) and varenicline (RR $3.99,95 \% \mathrm{Cl} 2.93$ to 5.44 ; $\mathrm{I}^{2}=\mathrm{n} / \mathrm{a}$; 1 study, 1510 participants) subgroups both provide evidence of higher rates of quitting in the pharmacotherapy groups. Removing studies at high risk of bias did not change the evidence that fast-acting NRT and varenicline were clearly beneficial. However, it is worthy of note that the number of studies and participants contributing to analyses varied considerably across subgroups.

\section{Quit attempts}

One study (Etter 2002; 534 participants) that used combination NRT in the intervention arm, reported data on the proportion making quit attempts but there was no clear evidence that either group made more quit attempts than the other (RR $1.04,95 \% \mathrm{Cl} 0.79$ to 1.37; Analysis 3.2).

\section{Pre-quit reduction}

We found seven studies that reported pre-quit smoking reduction measured dichotomously. Six of them used a reduction of at least $50 \%$ of baseline consumption as a cut-off (Bolliger 2000a; Etter 2002; Haustein 2002; Kralikova 2009; Rennard 2006; Wennike 2003), and one used a 75\% cut-off (Ebbert 2015). We decided to pool all seven studies together, as they all measured the incidence of a marked reduction in cigarette consumption; however, we split the studies into subgroups based on the type of pharmacotherapy used (combination NRT, fast-acting NRT and varenicline). The 
pooled estimate found that more participants reduced in the pharmacotherapy groups (RR $1.60,95 \% \mathrm{Cl} 1.22$ to $2.10 ;\left.\right|^{2}=71 \%$; 7 studies, 3472 participants; Analysis 3.3). Although overall heterogeneity was high, a test for subgroup differences was not significant $\left(1^{2}=\right.$ $52 \%, P=0.13)$.

Four studies (1502 participants) reported on mean reductions in consumption of cigarettes per day during the pre-quit period, all of which compared different types of NRT (combination NRT, nicotine patch, and fast-acting NRT) to placebo. Although subgroup differences were not significant, it was not possible to present a pooled result, as data from Hanson 2008 were duplicated across subgroups and would have been double-counted (Analysis 3.4). For the combination NRT subgroup the point estimate was MD 2.20 ( $95 \% \mathrm{Cl} 0.40$ to $4.00 ; 1^{2}=n / a ; 1$ study, 534 participants), for the nicotine patch subgroup MD $-0.10\left(95 \% \mathrm{Cl}-2.63\right.$ to $2.43 ; \mathrm{I}^{2}=\mathrm{n} / \mathrm{a} ; 1$ study, 70 participants), and for the fast-acting NRT subgroup MD $1.16(95 \% \mathrm{Cl}-0.09$ to $2.41 ; 1^{2}=0 \% ; 3$ studies, 898 participants).

Finally, two studies with three comparisons (Bolliger 2000a; Hanson 2008) reported on mean reduction of exhaled CO. Hanson 2008 was entered into the analysis twice (Analysis 3.5) as it had a group that received nicotine patches and a group that received nicotine gum. As previously, the same data from the unmatched placebo control group had to be entered as a comparator for both interventions, so we could not pool the data. We split the studies into two subgroups: 1) nicotine patch; 2) fast-acting NRT, with the two comparisons from Hanson 2008 split between the two groups. However, when we attempted to pool the two studies within the fast-acting NRT subgroup (Bolliger 2000a; Hanson 2008) we noted substantial heterogeneity $(12=87 \%)$. As a result we have not provided a pooled estimate (see Analysis 3.5 for individual study estimates).

\section{Pre-quit adverse effects}

Two studies (Bolliger 2000a; Shiffman 2009), including 3697 participants, reported sufficient data on the number of people reporting pre-quit AEs to allow analysis. However, pooling produced substantial heterogeneity $\left(I^{2}=89 \%\right)$, and we therefore deemed it inappropriate to report the pooled effect estimate (see Analysis 3.6 for point estimates for individual studies). Bolliger 2000a found no evidence of a between-group difference in the number of people reporting AEs and also found similar numbers of overall AEs reported in each arm, with slightly more reported in the placebo arm (Analysis 3.8). Shiffman 2009 found evidence that more people reported adverse events in the nicotine gum arm, but the most frequently reported adverse events were mild and characteristic of standard nicotine gum use, e.g. nausea, hiccups, heartburn. Caldwell 2016 reported the individual frequencies of a list of AEs, but did not report the number of people reporting any AE overall. In summary, after one day of abstinence significantly more participants in the active NRT group reported cough, scratchy throat, sore throat, lightheadedness, nausea and heartburn. These symptoms are all common, non-serious side effects of standard NRT use. There were no significant differences in the numbers reporting difficulty breathing, headache, chest discomfort, palpitations, vomiting, head-rush, jitter, itchy skin, red mark, sleep disturbance or other side effects. Two further studies (Caldwell 2016; Cook 2016) reported on the number of people who reported SAEs pre-quit. Cook 2016 reported no SAEs in any trial arm and Caldwell 2016 reported very low numbers of SAEs resulting in an RR of 7.28 (95\% Cl 0.38 to 140.28 ; 12 $=89 \%$; 2 studies, 762 participants) with substantial imprecision. In addition, Etter 2002 reported narratively that the difference in the rate of SAEs in the combination NRT and placebo groups was not statistically significant $(P=0.25)$, and that the reported SAEs were unlikely to be due to the treatment.

Caldwell 2016 and Haustein 2002 both reported on changes in withdrawal symptoms over the pre-quit period. In Caldwell 2016 groups had similar withdrawal scores up to one day after their quit day, whereas Haustein 2002 found that there was a statistically significant decrease in restlessness in the shorter active treatment group, and in difficulty concentrating in the longer placebo group.

\section{'Modality of reduction support' comparison}

\section{Abstinence}

Four studies investigated the effect of reduction-to-quit treatment modality on smoking cessation rates (Analysis 4.1). Jerome 1992 compared behavioural support added to self-help with self-help alone, Curry 1988 and Farley 2017 simply compared behavioural support with self-help, and Garcia 2000 included intervention arms that allowed for both types of comparison. Although the studies investigated two slightly different comparisons, we made the decision to pool them all together as evidence suggests that the effect of self-help is minimal when combined with behavioural support, as in Garcia 2000 and Jerome 1992 (Livingstone-Banks 2019). Pooling resulted in an RR of 1.99 (95\% Cls 1.21 to 3.27 ; $12=0 \%$; 4 studies, 296 participants; Analysis 4.1), suggesting a benefit of delivering reduction-to-quit interventions using behavioural support rather than written self-help materials. This effect persisted when the two studies judged to be at high risk of bias (Curry 1988; Farley 2017) were removed from the analysis ( $\mathrm{RR} 1.89,95 \% \mathrm{Cl} 1.03$ to 3.48 ; $\mathrm{I}^{2}=0 \%$; 2 studies, 163 participants), although there was increased imprecision.

\section{Pre-quit reduction}

Two studies reported on pre-quit mean reduction in cpd (Analysis 4.2). Pooling Garcia 2000 and Jerome 1992 provided evidence that a higher reduction in cpd occurred in the groups that received behavioural support (MD 7.00, $95 \% \mathrm{Cl} 3.50$ to $10.50 ; \mathrm{I}^{2}=0 \% ; 2$ studies, 107 participants), although the $\mathrm{Cl}$ indicates imprecision.

\section{'Length of reduction' comparison}

\section{Abstinence}

Two studies (Farley 2017; Haustein 2002), including 453 participants, provided adequate data for this outcome. When we pooled them, we did not find evidence of a clear difference between the quit rates generated by a longer (16 weeks in Farley 2017; six to nine months in Haustein 2002) versus a shorter (four weeks in both Farley 2017 and Haustein 2002) reduction period. Although the point estimate favoured a shorter period, the $\mathrm{Cl}$ also incorporated the null (RR $0.22,95 \% \mathrm{Cl} 0.05$ to $1.01 ; \mathrm{I}^{2}=\mathrm{n} / \mathrm{a}$ ). As there were no quitters in Farley 2017, the estimate was based solely on Haustein 2002 (Analysis 5.1). We judged both studies contributing to this analysis to be at high risk of bias, so it was not possible to conduct a sensitivity analysis to assess the potential impact of this.

\section{Pre-quit reduction}

Only Haustein 2002 (385 participants) reported useable data relevant to pre-quit smoking reduction, by reporting the number of participants who successfully reduced by $50 \%$ or more of their base- 
line cpd consumption by trial arm. The evidence suggested that there was a benefit of the shorter four-week reduction period on reduction in cigarette consumption, compared with the longer sixto-nine-month reduction period ( $\mathrm{RR} 0.58,95 \% \mathrm{Cl} 0.39$ to $0.86 ; \mathrm{I}^{2}=$ $\mathrm{n} / \mathrm{a}$; Analysis 5.2).

\section{Pre-quit adverse effects}

Only Haustein 2002 reported on any adverse effects of a longer versus a shorter pre-quit reduction period. They did this by reporting on withdrawal symptoms between baseline and month four of the study. This time point came after the quit day in the shorter reduction group, but provides a reasonable time point for comparison. The shorter placebo group experienced statistically significant decreases in urge to smoke, whereas there was a statistically significant decrease in difficulty concentrating in the longer placebo group. There were no significant within-group changes in any of the other symptoms (anxiety, increased appetite, insomnia, irritability/frustration) between baseline and month four. Between-group significance testing was not reported (Analysis 5.3).

\section{'More structured versus less structured reduction' comparison}

\section{Abstinence}

Two studies (727 participants) measured abstinence at long-term follow-up in studies comparing more structured and in-depth reduction-to-quit advice with less structured and in-depth advice (Cinciripini 1995; Cummings 1988). However, meta-analysis found that the variance between study effects was high, making pooling inappropriate $\left(I^{2}=84 \%\right)$. For individual study effect estimates see Analysis 6.1.

\section{Quit attempts}

Cinciripini 1995 and Cummings 1988 also reported on the number of quit attempts made by trial arm. For this outcome heterogeneity was moderate $(12=57 \%$; Analysis 6.2$)$. There was no evidence of a clear difference between more versus less structured reduction-to-quit advice (RR $0.99,95 \% \mathrm{Cl} 0.82$ to 1.18 ; 2 studies, 727 participants).

\section{Pre-quit reduction}

Only Cinciripini 1995 (65 participants) reported on pre-quit smoking reduction for this comparison. The evidence suggested that neither more nor less structured advice resulted in a greater reduction in cpd prior to the quit day (MD 1.88, $95 \% \mathrm{Cl}-3.03$ to $6.79 ; \mathrm{I}^{2}=\mathrm{n} / \mathrm{a}$; Analysis 6.3); however, the effect was imprecise.

\section{Pre-quit adverse effects}

Cinciripini 1995 measured the occurrence of nicotine withdrawal symptoms and found that these increased in both the morestructured intervention group and the less-structured intervention group. The increase was slightly higher in the less-structured group, but the difference between groups was not tested for statistical significance.

\section{Additional behavioural smoking cessation (SC) components}

\section{Abstinence}

Four studies tested the effect of adding more behavioural support or additional components of behavioural support to a reduction-to-quit intervention. The nature of the additional support var- ied across all four studies. Garcia 2000 investigated the effect of twice-weekly versus weekly behavioural support; Chan 2011 investigated the effect of additional NRT adherence counselling; Malott 1984 investigated the effect of adding a peer-support component; and Nicki 1984 investigated the effect of motivational 'self-talk'. Pooling these studies to judge the effect of adding extra behavioural support to a reduction-to-quit intervention found no evidence of an overall effect on smoking cessation rates (RR $0.79,95 \% \mathrm{Cl} 0.34$ to 1.84; 4 studies, 1033 participants); heterogeneity was moderate ( ${ }^{2}$ $=67 \%$ ), and the $95 \% \mathrm{Cl}$ indicated the potential for both harm and benefit. We rated none of the studies at high risk of bias, so sensitivity analysis was not necessary. For individual study effect estimates see Analysis 7.1. Only Chan 2011 found evidence to suggest a beneficial effect of an added NRT adherence intervention (RR 1.73, 95\% $\mathrm{Cl} 1.09$ to $2.74 ; \mathrm{I}^{2}=\mathrm{n} / \mathrm{a}$; 928 participants).

\section{Pre-quit reduction}

Garcia 2000 (56 participants) measured whether twice-weekly behavioural support for reduction resulted in a greater reduction in cpd than weekly support. The resulting effect estimate was imprecise, suggesting the possibility of both a notably greater reduction and a slightly smaller reduction in the twice-weekly arm compared with the weekly arm (MD 3.40, $95 \% \mathrm{Cl}-0.18$ to $6.98 ; \mathrm{I}^{2}=\mathrm{n} / \mathrm{a}$; Analysis 7.2).

\section{'Behavioural reduction versus nicotine fading' comparison}

Three small studies (Dooley 1992; Gariti 2004; Nicki 1984) compared a reduction-to-quit study arm versus one that included nicotine-fading treatment, with participants asked to switch to cigarette brands with nicotine levels lower than their usual brand, either alone or alongside a reduction-to-quit intervention.

\section{Abstinence}

All three studies reported long-term smoking abstinence rates. Each study tested a slightly different comparison (Analysis 8.1):

- Dooley 1992 (75 participants) directly compared a reduction-toquit intervention to a nicotine-fading intervention, resulting in an RR of $0.97\left(95 \% \mathrm{Cl} 0.31\right.$ to $\left.3.09 ; \mathrm{I}^{2}=\mathrm{n} / \mathrm{a}\right)$. The confidence interval indicates that behavioural reduction could result in both substantial benefit and harm when compared to nicotine fading.

- Nicki 1984 (49 participants) tested the effect of adding a behavioural reduction-to-quit intervention to a nicotine-fading intervention, and found a potentially large benefit of offering reduction to quit alongside nicotine fading (RR $4.32,95 \% \mathrm{Cl} 1.04$ to $\left.17.98 ; 1^{2}=n / a\right)$.

- Gariti 2004 (60 participants) tested the effect of adding a nicotine-fading intervention to a behavioural reduction-to-quit intervention, which resulted in a RR of 1.33 (95\% Cl 0.33 to 5.45 ; 12 $=\mathrm{n} / \mathrm{a}$ ). Although the point estimate suggests an increase in cessation when fading is used alongside behavioural reduction, the $\mathrm{Cl}$ indicates substantial imprecision.

\section{Pre-quit reduction}

Only Gariti 2004 (60 participants) measured and reported sufficient data for this outcome, by reporting reductions in cotinine levels during the pre-quit period. Although the point estimate suggested a greater reduction in cotinine levels in the behavioural reduction-only arm, the effect was very imprecise, with the $\mathrm{Cl}$ also sug- 
gesting the potential for a slightly greater reduction in cotinine in the behavioural reduction plus nicotine-fading arm (MD 53.00, 95\% $\mathrm{Cl}-2.54$ to $108.54 ; \mathrm{I}^{2}=\mathrm{n} / \mathrm{a}$; Analysis 8.2).

\section{Pre-quit adverse effects}

Gariti 2004 was also the only study relevant to the nicotine-fading comparisons to report on adverse effects during the pre-quit period. No SAEs were reported in either trial arm and no between-group differences in nicotine withdrawal (Analysis 8.3). Withdrawal scores were described as consistently low across trial arms, which was deemed to be indicative of slight withdrawal.

\section{Other comparisons}

A further four comparisons were tested by one each of the included studies. All of these studies reported long-term smoking abstinence (Analysis 9.1), but only one reported any further outcomes of interest to this review (Glasgow 1989). The comparisons and their reported outcomes are as follows.

\section{Aerobic exercise versus health education}

Blevins 2016 (61 participants) randomised participants to either a programme of aerobic exercise or a programme of general health education, all related to the effects of smoking. In both groups participants were asked to reduce by one to two cpd until their quit day four weeks post-baseline. The point estimate favoured the exercise intervention, but the $\mathrm{Cl}$ was very wide, providing evidence of both substantial benefit and harm (RR 4.13, $95 \% \mathrm{Cl} 0.49$ to $34.89 ; \mathrm{I}^{2}=\mathrm{n} / \mathrm{a}$ ).

\section{Reduction to zero versus partial reduction to quit}

Flaxman 1978 (32 participants) included two groups advised to reduce their smoking before quitting. One group was asked to reduce down to zero cpd as a means to quit completely, whereas the other was advised to reduce by $50 \%$ of their baseline consumption and then to stop smoking altogether. The effect estimate was imprecise and did not provide clear evidence of a benefit of either approach (RR $2.00,95 \% \mathrm{Cl} 0.60$ to $6.64 ; \mathrm{I}^{2}=\mathrm{n} / \mathrm{a}$ ).

\section{Contingent versus non-contingent vouchers for reduction}

Rohsenow 2016 (340 participants) investigated the effect of providing vouchers contingent on pre-quit smoking reduction (measured using exhaled (C) and then abstinence, in comparison with providing vouchers non-contingent on reduction and cessation. This study also produced an imprecise effect estimate, with no evidence that either approach resulted in superior quit rates (RR 1.95, 95\% Cl 0.50 to $\left.7.68 ; 1^{2}=n / a\right)$.

\section{Greater reduction targets and a choice of abstinence or controlled smoking versus minimal reduction and a clear abstinence target}

Glasgow 1989 (66 participants) included two groups of participants with reduction occurring in each. In the first group (the cessation-controlled condition) participants were asked to carry out nicotine fading and also to reduce their number of cigarettes smoked per day to between $33 \%$ and $50 \%$ of their baseline rate. They were then encouraged to stop smoking altogether, but were also told that they could choose to continue smoking at a reduced rate. The second group (the 'abstinence-based' condition) were provided with an explicit quit date and asked to carry out nicotine fading and also to target individual cigarettes to eliminate (with- out reduction targets) as practice for coping without any cigarettes. This resulted in an RR of $1.06\left(95 \% \mathrm{Cl} 0.36\right.$ to $\left.3.14 ; \mathrm{I}^{2}=\mathrm{n} / \mathrm{a}\right)$.

Glasgow 1989 also measured the amount of pre-quit reduction in each arm, using continuous measures of both cpd and exhaled CO. In neither case was there clear evidence of a difference in reduction rates between the two groups. The MD for cpd was $-1.40(95 \% \mathrm{Cl}$ -6.43 to $3.63 ; \mathrm{I}^{2}=\mathrm{n} / \mathrm{a} ; 66$ participants), and for $\mathrm{CO}$ was $3.40(95 \% \mathrm{Cl}$ -4.59 to $11.39,1^{2}=n / a ; 66$ participants).

No SAEs were reported during the pre-quit period across either study group, and the study narratively reports that there were no significant between-group differences in pre-quit withdrawal symptoms (Analysis 9.4).

\section{DISCUSSION}

\section{Summary of main results}

This review includes 51 trials. It focuses specifically on interventions that advised people who smoke to reduce the amount they smoked before quitting completely. Six trials compared a reduction-to-quit intervention with no smoking cessation intervention, 27 compared a reduction-to-quit intervention with an abrupt quitting intervention (i.e. a smoking cessation intervention that did not advise participants to reduce their cigarette consumption prequit), and 29 studies compared different reduction-to-quit interventions. The most common characteristic of reduction-to-quit interventions tested was pharmacotherapy, used as an aid to reduction (12 studies). Other characteristics tested were length of the reduction period, modality of the support provided, how structured the reduction support was, and the amount of behavioural support provided.

Pooling smoking cessation data for all six trials comparing reduction-to-quit interventions with no smoking cessation intervention resulted in a no clear evidence that reducing to quit interventions resulted in better quit rates than no smoking cessation treatment. We judged this result to be of low certainty, due to risk of bias, unexplained heterogeneity, and imprecision; the $\mathrm{Cl}$ incorporated both clinically meaningful harm, as well as substantial benefit of the intervention. The lack of evidence of an effect should therefore not be interpreted as proof that reduction-to-quit interventions will result in equal quit rates to no treatment, as future evidence is very likely to change our interpretation of the result. There was some evidence, from a small number of trials, that reduction-to-quit interventions resulted in more quit attempts and higher rates of smoking reduction than no treatment.

Abrupt quitting interventions are generally recommended in clinical treatment guidelines (e.g. Fiore 2008; NICE 2018), and are therefore deemed to be standard behavioural support for smoking cessation. Our analysis of reduction-to-quit interventions versus abrupt quitting interventions provided moderate-certainty evidence that neither resulted in superior long-term quit rates. We did not judge this evidence as being of high certainty, due to some imprecision of the effect. The $\mathrm{Cl}$ indicated that reduction-to-quit interventions could potentially lead to slightly lower quit rates than abrupt quitting, as well as slightly higher quit rates. A statistically significant subgroup analysis found some evidence that reduction-to-quit interventions may be more effective than abrupt quitting interventions if varenicline is used as an aid to reduction; however, this result was based on only one study in 314 people, and so 
should be treated cautiously. Additional subgroup analyses found no evidence that other characteristics of reduction-to-quit interventions moderated the relative effect of reduction to quit versus abrupt quitting interventions on smoking cessation.

Our comparison of reduction-to-quit interventions supported by pharmacotherapy versus placebo or no pharmacotherapy found low-certainty evidence that using pharmacotherapy to aid reduction to quit resulted in higher quit rates. We downgraded the evidence for imprecision, as the $\mathrm{Cl}$ indicated a potentially weak and large effect, and for inconsistency, as there was substantial statistical heterogeneity. This was partially but not completely explained by a subgroup analysis, which found a positive effect of using varenicline (moderate certainty) and fast-acting NRT (moderate certainty), but not of using nicotine patches (very low certainty), combination NRT (low certainty) or bupropion (low certainty) as an aid. However, due to the low and very low certainty of the latter evidence it is likely that future trials may change the overall interpretation of those effects.

Within-study comparisons investigating the impact of other intervention characteristics on quit rates found some evidence that reduction-to-quit interventions may be more effective when delivered using behavioural support as opposed to self-help materials; however, no other characteristics of reduction-to-quit interventions resulted in superior long-term cessation rates. Evidence contributing to secondary outcomes was sparse, and in many cases imprecision and inconsistency made it hard to draw any clear conclusions, but there was some indication that reduction interventions did lead to reductions in both cigarette consumption and smoke inhalation. Trials that measure serious adverse events (SAEs) in the pre-quit period generally found that there were either none reported or that rates were low and well-balanced between trial arms. Two trials investigating the effects of adding fast-acting NRT to a reduction-to-quit intervention found evidence of higher rates of adverse events (AEs) in the NRT groups. However, the AEs reported were mild and were typically associated with the use of NRT for a quit attempt, which evidence suggests is both safe and effective (Hartmann-Boyce 2018).

\section{Overall completeness and applicability of evidence}

The searches conducted for this review were broad, in our attempt to find any study that made any mention of reduction. As well as medical databases, we also searched trial registers to identify any ongoing or completed but unpublished, registered studies. We therefore feel confident in our search approach. However, behavioural smoking cessation interventions are not always well-described, and it is possible that we may have missed studies if they did not make it clear that smoking reduction advice was a component of the intervention. In addition, this area of investigation first became popular in the 1970s, long before trial registration began, so it is therefore possible that studies that we do not know about were carried out and remain unpublished.

The studies identified in this review were mainly conducted in the USA, and the others all took place in other high-income or higher middle-income countries. Most studies were carried out in the general population and so may not be applicable to populations with specific requirements or particularly high dependence. As reduction to quit may be an attractive cessation strategy for populations who find it harder to quit, such as those with mental health prob- lems, substance abuse issues, or experiencing homelessness, studies carried out in these specific population groups would be useful.

It was an eligibility criterion for the review that each study had to assess long-term abstinence, so most studies were able to contribute cessation data to the relevant comparisons. However, for secondary outcomes the numbers of studies and participants contributing to a comparison were sparse, and further research could strengthen or change findings.

\section{Certainty of the evidence}

Of the 51 studies included in this review, we judged five to be at low risk of bias for all domains, and 18 to be at high risk in one or more domains. In many cases, we rated studies at an unclear risk because they did not report key information. In these cases, it is impossible to know whether these studies were at any risk of bias or whether the information was simply not reported. To investigate the potential impact on results of studies that we judged to be at high risk of bias, we removed these studies in sensitivity analyses. This did not affect our interpretation of results.

We assessed the certainty of the evidence by creating 'Summary of findings' tables for the reduction versus no-treatment comparison, the reduction versus abrupt comparison, and the reduction with pharmacotherapy versus reduction-alone comparison. We carried out GRADE ratings for the smoking cessation outcome for each one (Summary of findings for the main comparison; Summary of findings 2; Summary of findings 3).

We judged evidence contributing to the reduction-to-quit versus no smoking cessation treatment comparison to be very low certainty, as most studies were rated at high risk of bias, there were moderate levels of heterogeneity which remained unexplained, and there was considerable imprecision (Summary of findings 2). This is because there were low numbers of quitters across the contributing studies overall and $\mathrm{Cls}$ spanned both potential harm and considerable benefit. We judged the certainty of the evidence for the reduction-to-quit versus abrupt quitting comparison as moderate, only downgrading due to some slight imprecision in the effect (Summary of findings for the main comparison). Cls incorporated both a small harm and a benefit of the reduction intervention when compared with the abrupt approach. Although imprecision was relatively low, the absolute quit rates demonstrate that this could equate to $2 \%$ higher quit rates in people who reduce to quit compared with those who quit abruptly. Potential small increases in smoking cessation effects such as this can have appreciable health impacts when interventions are implemented at a population level. We would therefore require confidence intervals tighter than this to conclude that the effects of abrupt quitting and reduction to quit were equivalent. If estimated differences in absolute quit rates fall to less than $2 \%$ in future updates of this review then we would deem it appropriate to conclude with high certainty that the two approaches are likely to result in similar quit rates. Finally, we rated the evidence contributing to the reduction with pharmacotherapy versus reduction-alone comparison as low certainty, also due to inconsistency between study effects and imprecision. However, because there was evidence of subgroup differences that may have implications for treatment delivery, we also carried out a GRADE assessment of the separate subgroups (nicotine patch; fastacting NRT; combination NRT; varenicline; and bupropion). Ratings ranged from very low to moderate certainty. We downgraded all subgroup comparisons due to imprecision, with the subgroup es- 
timate deemed to be of very low certainty (nicotine patch) downgraded by two levels due to imprecision, and by one level due to risk of bias. This was because we judged the only study in the comparison to be at high risk of bias; participants in the control group did not receive a placebo, making blinding impossible.

\section{Potential biases in the review process}

To conduct this review we followed standard Cochrane methods and consider the review process used to be robust. For 'Risk of bias' outcome assessment, we followed the standard methods used for Cochrane Tobacco Addiction Review Group cessation reviews; we assessed both performance and detection bias for studies testing a pharmacological intervention, and detection bias only for those studies that tested a behavioural intervention. Our search strategy included searches of trial registries in an attempt to capture unpublished and ongoing studies. However, as this was an active research area more than 40 years ago there is the possibility that there are unreported studies that would not have been registered. We are aware of three studies comparing reduction to quit to abrupt quitting where measurement of long-term cessation outcomes were planned, but these have not been reported (Cinciripini 2006; NCT00158158; Riley 2001). We can not know whether this is due to non-significant results; however, even if that were the case, this would strengthen the interpretation of our overall result rather than changing it. Two studies reported study quit rates overall, but not by trial arm (Glasgow 1978; Hanson 2008). Due to the age of the studies investigators were unable to provide the individual group data when we contacted them during the review process, but both studies reported narratively that there was no significant difference in quit rates between groups. Data from Glasgow 1978 should have contributed to the modality analysis, comparing reduction delivered through self-help with reduction delivered through behavioural support, and Hanson 2008 should have contributed to the nicotine patch subgroup of the analysis investigating the use of pharmacotherapy to aid reduction. In the latter case including these data would not have influenced the interpretation of our results; although there is a chance that including Glasgow 1978 in the modality analysis may have weakened the positive effect of behavioural support on abstinence rates. However, this trial had a very small sample size $(N=62)$ and so any change in the effect would likely be minimal.

Two meta-analyses included enough studies to allow us to create a useful funnel plot. These were 1) reduction to quit versus abrupt quitting (Figure 3) and 2) reduction with pharmacotherapy versus reduction alone (Figure 4). The second plot showed no indication of publication bias, whereas the first does suggest a lack of smaller studies favouring abrupt quitting. This could indicate publication bias, but as both treatments being tested in this comparison were active it is less likely that this is the case than if the comparator were a placebo or no treatment. Additionally, as this comparison included a number of larger studies that found no clear difference in quit rates between the methods, even if there were additional unpublished smaller studies favouring abrupt quitting they would be unlikely to change the overall estimate or our interpretation of the effect. 
Figure 3. Funnel plot of comparison: 2 Reduction to quit versus abrupt quitting, outcome: 2.1 Abstinence.

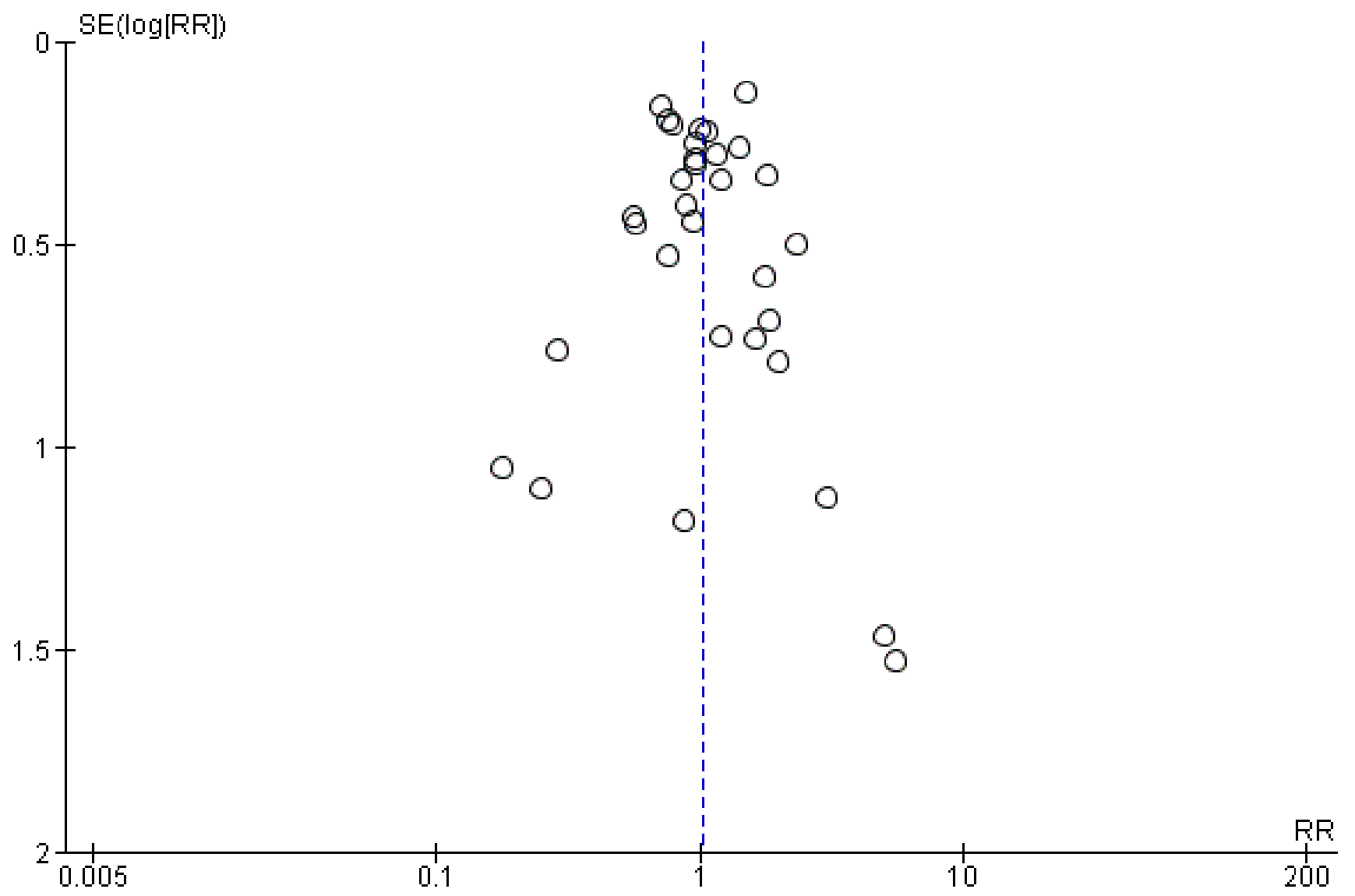


Figure 4. Funnel plot of comparison: 3 Reduction + pharma vs reduction alone, outcome: 3.1 Abstinence.

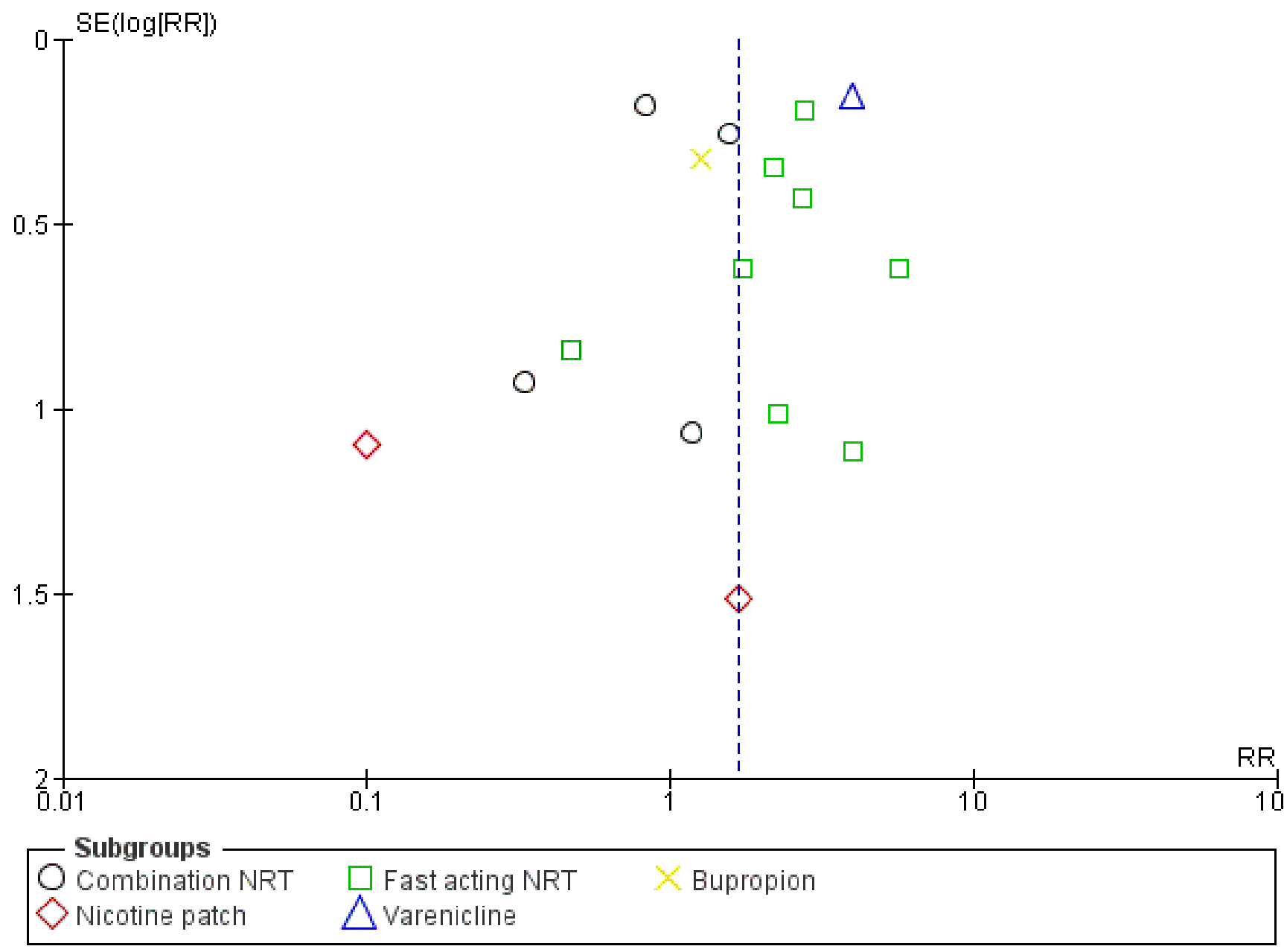

The significant unexplained heterogeneity detected within some of the analyses is likely to have been caused by substantial variation across the characteristics of both the intervention and comparator arms. Despite dividing studies into prespecified comparisons and subgroups in an attempt to reduce and explain some of this variation, key differences remained in the components of reduction-toquit interventions and their comparators. Studies typically provided only limited explanation of the content of interventions. Where some studies only specified that reduction was advised, it was difficult to know whether instructions were minimal or whether more specific instructions were provided but not described in trial reports. We therefore categorised the studies as well as possible, given the information provided.

\section{Agreements and disagreements with other studies or reviews}

This review supersedes a previous Cochrane Review which focused on the reduction-to-quit versus abrupt quitting comparison only (Lindson-Hawley 2012). This review more than doubles the number of studies included in that review for the relevant comparison, and strengthens the original findings. The previous estimate for the relative effect of reduction over abrupt quitting was RR 0.94 (95\% Cl 0.79 to $1.13 ;\left.\right|^{2}=14 \% ; 10$ studies, 3760 participants), compared with RR 1.01 (95\% Cl 0.87 to 1.17 ; $\left.\right|^{2}=29 \%$; 22 studies, 9219 participants) for this update. Both estimates suggest that reduction to quit does not substantially improve or worsen quit rates, compared with quitting abruptly.

A review investigating smoking reduction interventions only in people unwilling to quit included some of the studies also included in this review, but also included some of the studies we excluded as they did not explicitly aim to help participants to quit smoking completely (Wu 2015). The findings suggest that there is a benefit of providing smoking-reduction support with pharmacotherapy when compared to no intervention (RR $1.93,95 \% \mathrm{Cl} 1.41$ to 2.64; $12=46 \%$; 3033 participants), although this positive effect was not found in the one study that compared reduction support alone with no treatment. This is in contrast to the comparable analysis in our review, which made it difficult for us to draw conclusions on the efficacy of reduction-to-quit interventions compared with no treatment. Wu 2015 also included an analysis investigating the effect of pharmacotherapy (varenicline or NRT or bupropion) added to smoking reduction advice, and comparing smoking reduction advice with pharmacotherapy to other types of smoking cessation advice (defined in this review as abrupt quitting) with pharmacotherapy. The results of these analyses both supported the findings of this review. Evidence suggested that reduction support with med- 
ication increased long-term quit rates when compared with reduction support plus placebo (RR $1.97,95 \% \mathrm{Cl} 1.44$ to $2.71 ; \mathrm{I}^{2}=52 \%$; 4678 participants), and a subgroup analysis found evidence of a positive effect of pharmacotherapy in the NRT (not split into patch and fast-acting varieties) and varenicline groups, but no evidence of an effect in the bupropion group. There was no evidence that either reduction support or abrupt quitting support resulted in superior quit rates (RR $0.93,95 \% \mathrm{Cl} 0.44$ to $2.00 ; 1^{2}=40 \%$; 476 participants); this result was more imprecise than our analysis, due to the much smaller sample size.

Another review investigating fast-acting NRT (gum or inhalator) as an aid to reduction-to-quit interventions only in smokers unmotivated to quit (Moore 2009) also found that the use of fast-acting NRT resulted in increased quit rates (RR $1.99,95 \% \mathrm{Cl} 1.01$ to 3.91 ; 1833 participants). This review also meta-analysed seven trials in various safety analyses and found no evidence for a difference in deaths (odds ratio (OR) $1.00,95 \% \mathrm{Cl} 0.25$ to 4.02 ), serious adverse events (OR $1.16,95 \% \mathrm{Cl} 0.79$ to 1.50 ), or discontinuation of NRT due to adverse events (OR $1.25,95 \% \mathrm{Cl} 0.64$ to 2.51 ) between the NRT and placebo interventions (Moore 2009). Similar to the findings of this review, the only adverse event that was more common in the NRT interventions was nausea (OR $1.69,95 \% \mathrm{Cl} 1.21$ to 2.36 ), which is a mild but common side effect of NRT. Taken with other safety data on concurrent smoking and use of NRT (Fagerström 2002b; Lindson 2019), there appears to be no reason to advise against using NRT to aid reduction, and using it will increase long-term cessation. Varenicline and bupropion have always been recommended for use whilst still smoking before a quit attempt, and safety considerations are therefore the same as those for standard use, and are covered by the corresponding Cochrane Reviews (Cahill 2016 and Hughes 2014 respectively).

General population surveys (West 2001; Cheong 2007) have found that reducing to quit is not as effective as abrupt quitting, but these studies differ from the RCTs included in this meta-analysis in ways that may explain the difference in outcomes. Many participants reducing to quit in the RCTs were provided with some instructions as to how to reduce (for example, setting quotas of cigarettes by which to reduce, and setting time intervals at which participants could smoke). We do not know how participants included in the observational studies chose to reduce, and they may have varied in their levels of successful pre-quit reduction. As some national guidelines do not explicitly recommend reducing consumption before quitting (Fiore 2008; NICE 2018), healthcare services are more likely to offer abrupt quitting as a cessation method. This means that those participants who chose reduction to quit in the observational studies were less likely to have benefited from any kind of support whilst quitting (behavioural or self-help materials). Evidence suggests that this type of support improves people's chances of quitting, so the lower quit rates may not have been a result of reduction alone (Lancaster 2017; Livingstone-Banks 2019). Moreover, participants in these surveys chose whether they quit abruptly or by reducing first; there is evidence that people who have higher motivation to quit choose to quit abruptly whilst those with lower motivation choose to reduce (Peters 2007). However, within the trials we reviewed, randomisation equalised participants' motivation between the reduction and abrupt quitting arms.

\section{AUTHORS' CONCLUSIONS}

\section{Implications for practice}

- Evidence suggests that neither reducing smoking to quit nor quitting abruptly results in superior quit rates; people could therefore be given a choice of how to quit, and support provided to people who would specifically like to reduce their smoking before quitting.

- There is some evidence that using fast-acting NRT or varenicline whilst reducing smoking before a quit day may help more people to quit smoking than reducing to quit without these pharmacological aids.

- There is some evidence that providing behavioural support to reduce to quit helps more people to stop smoking than providing people with self-help resources only.

- There is currently no clear evidence in support of certain reduction methods over others; however, further evidence could change this conclusion.

\section{Implications for research}

- Future studies investigating the effect of reduction-to-quit interventions should aim to minimise the number of co-interventions and match these across intervention and comparator groups to reduce confounding. Studies should also provide detailed descriptions of the interventions implemented in all study groups, to allow the investigation of specific intervention components.

- Further research should focus on investigating which are the most effective reduction-to-quit methods, as high-quality evidence in this area is currently sparse. This would help to implement reduction interventions in the most effective ways. Areas of focus should include the most effective pharmacotherapies to use, the optimal length of reduction methods and reduction targets, whether a quit date should be set in advance, and specific instructions on reduction methods, e.g. increasing inter-cigarette intervals, that may maximise success.

- Reducing smoking before quitting may appeal to populations who find it particularly difficult to quit smoking, such as people with mental health problems. However studies in these populations are limited. Future studies should investigate reduction to quit in populations who have higher rates of smoking and who find it harder to quit.

- Trials should assess abstinence at least six months following baseline. Secondary outcomes such as quit attempts and reduction should also be measured, to see if this can provide insight into how reduction-to-quit interventions may work. This information may be helpful when developing optimal reduction-toquit interventions. Studies should also monitor any adverse effects of interventions, such as withdrawal symptoms, adverse events and serious adverse events. These are specifically of interest during the pre-quit period, to investigate whether they mediate the effect of the intervention on abstinence.

\section{ACKNOWLEDGEMENTS}

The authors would like to thank Professor John Hughes (University of Vermont), who was an author on previous versions of this review and protocol (title: Reduction versus abrupt cessation in smokers who want to quit), and provided guidance on the protocol for this review. We also gratefully acknowledge the help of Yuan Chi in translating a paper published in the Chinese language to the English lan- 
guage to confirm eligibility and aid data extraction, and the help of Lydia Jones in translating a paper published in the German language to the English language to aid eligibility assessment. We also thank the many authors who responded to information requests during this review process.

Thanks also to Jonathan Livingstone-Banks, Jamie Hartmann-Boyce and Robert West for offering editorial support, to Joanna Ting Wai Chu, PhD, The National Institute for Health Innovation, University of Auckland and Professor Billie Bonevski, University of Newcastle, Australia for providing peer review, and to Lee Bromhead for providing consumer review.

$\mathrm{NL}$ is Managing Editor and PA is Co-ordinating Editor for the Cochrane Tobacco Addiction Group. JMOM provides statistical sup- port. This project was supported by the National Institute for Health Research (NIHR), via Cochrane Infrastructure and Cochrane Programme Grant funding to the Cochrane Tobacco Addiction Group. PA is an NIHR senior investigator and funded by NIHR Oxford Biomedical Research Centre and CLAHRC. JMOM is partly funded by the National Institute for Health Research (NIHR) Oxford Biomedical Research Centre (BRC), and by the National Institute for Health Research (NIHR) Community Healthcare Medtech and In Vitro Diagnostics Cooperative (MIC). The views and opinions expressed therein are those of the authors and do not necessarily reflect those of the Systematic Reviews Programme, NIHR, NHS, or the Department of Health and Social Care. 


\section{REFERE N CE S}

\section{References to studies included in this review}

Blevins 2016 \{published data only\}

Blevins CE, Farris SG, Brown RA, Strong DR, Abrantes AM. The role of self-efficacy, adaptive coping, and smoking urges in longterm cessation outcomes. Addictive Disorders \& Their Treatment 2016;15(4):183-9.

\section{Bolliger 2000a \{published data only\}}

Bolliger CT, Zellweger JP, Danielsson T, Van Biljon X, Robidou A, Westin $A$, et al. Influence of long-term smoking reduction on health risk markers and quality of life. Nicotine \& Tobacco Research 2002;4(4):433-9.

* Bolliger CT, Zellweger JP, Danielsson T, Van Biljon X, Robidou A, Westin $A$, et al. Smoking reduction with oral nicotine inhalers: double blind, randomised clinical trial of efficacy and safety. BMJ 2000;321(7257):329-33.

Brockway 1977 \{published data only\}

Brockway BS, Kleinmann G, Edleson J, Gruenewald K. Non aversive procedures and their effect on cigarette smoking. Addictive Behaviors 1977;2(2-3):121-8.

\section{Caldwell 2016 \{published data only\}}

ACTRN12609000481279. Nicotine inhaler plus nicotine patch for smoking cessation [Pulmonary nicotine inhaler plus nicotine transdermal patch for 6-month smoking cessation in heavy smokers]. www.anzctr.org.au/Trial/Registration/ TrialReview.aspx?id=308032 (first received 18 June 2009).

* Caldwell BO, Crane J. Combination nicotine metered dose inhaler and nicotine patch for smoking cessation: a randomized controlled trial. Nicotine \& Tobacco Research 2016;18(10):1944-51.

\section{Carpenter 2003 \{published data only\}}

Carpenter MJ, Hughes JR, Keely JP. Effect of smoking reduction on later cessation: a pilot experimental study. Nicotine \& Tobacco Research 2003;5(2):155-62.

\section{Carpenter 2004 \{published data only\}}

* Carpenter MJ, Hughes JR, Solomon LJ, Callas PW. Both smoking reduction with nicotine replacement therapy and motivational advice increase future cessation among smokers unmotivated to quit. Journal of Consulting and Clinical Psychology 2004;72(3):371-81.

Hughes JR, Carpenter MJ. Smoking reduction with NRT increases, not decreases, future cessation. Society for Research on Nicotine and Tobacco 5th European Meeting; 2003 Nov 20-22; Padua, Italy. 2003.

\section{Chan 2011 \{published data only\}}

* Chan SS, Leung DY, Abdullah AS, Wong VT, Hedley AJ, Lam T$\mathrm{H}$. A randomized controlled trial of a smoking reduction plus nicotine replacement therapy intervention for smokers not willing to quit smoking. Addiction 2011;106(6):1155-63. [DOI: 10.1111/j.1360-0443.2011.03363.x]
Cheung YT, Lam TH, Leung DY, Abdullah AS, Chan SS. Nicotine replacement therapy to aid gradual cessation in smokers with no intention to quit: Association between reduction quantity and later abstinence. Preventive Medicine Reports 2015;2(2):196-201.

Lam T-H, Chan SS, Abdullah AS, Taam Wong V, Chan AY, Hedley AJ. Smoking reduction intervention for smokers not willing to quit smoking: A randomised controlled trial. Hong Kong Medical Journal 2012;18(3):4-8.

Lam TH, Cheung YT, Leung DY, Abdullah AS, Chan SS.

Effectiveness of smoking reduction intervention for hardcore smokers. Tobacco Induced Diseases 2015;13(1):9-15.

NCT00563329. Smoking reduction intervention for smokers not willing to quit smoking: a randomised control trial. clinicaltrials.gov/ct2/show/NCT00563329 (first received 26 Nov 2007).

\section{Cinciripini 1995 \{published data only\}}

${ }^{\star}$ Cinciripini PM, Lapitsky L, Seay S, Wallfisch A, Kitchens K, Van Vunakis H. The effects of smoking schedules on cessation outcome: can we improve on common methods of gradual and abrupt nicotine withdrawal?. Journal of Consulting and Clinical Psychology 1995;63(3):388-99.

Cinciripini PM, Wetter DW, McClure JB. Scheduled reduced smoking: effects on smoking abstinence and potential mechanisms of action. Addictive Behaviors 1997;22(6):759-67.

\section{Cinciripini 2006 \{published data only\}}

* Cinciripini PM, Lam C, Blalock JA, Robinson J, Wetter DE, Baile W. Does scheduled reduced smoking have a place among smoking cessation treatments?. Society for Research on Nicotine and Tobacco 12th Annual Meeting; 2006 Feb 15-18; Orlando, USA. 2006:14.

\section{Cook 2016 \{published data only\}}

* Cook JW, Collins LM, Fiore MC, Smith SS, Fraser D, Bolt DM, et al. Comparative effectiveness of motivation phase intervention components for use with smokers unwilling to quit: a factorial screening experiment. Addiction 2016;111(1):117-28.

NCT01122238. Identifying treatments to motivate smokers to quit. clinicaltrials.gov/ct2/show/NCT01122238 (first received 13 May 2010).

\section{Cummings 1988 \{published data only\}}

Cummings KM, Emont SL, Jaen C, Sciandra R. Format and quitting instructions as factors influencing the impact of a selfadministered quit smoking program. Health Education Quarterly 1988;15(2):199-216.

\section{Curry 1988 \{published data only\}}

Curry SJ, Marlatt GA, Gordon J, Baer JS. A comparison of alternative theoretical approaches to smoking cessation and relapse. Health Psychology 1988;7(6):545-56. 
Dooley 1992 \{published data only\}

Dooley RT, Halford WK. A comparison of relapse prevention with nicotine gum or nicotine fading in modification of smoking. Australian Psychologist 1992;27(3):186-91.

\section{Ebbert 2015 \{published data only\}}

* Ebbert JO, Hughes JR, West RJ, Rennard SI, Russ C, McRae TD, et al. Effect of varenicline on smoking cessation through smoking reduction: a randomized clinical trial. JAMA 2015;313(7):687-94.

Fagerström K. Varenicline can help smokers to stop smoking by gradual reduction. BMJ Evidence-Based Medicine 2015;20(4):133.

Nakamura M, Abe M, Ohkura M, Treadow J, Yu CR, Park PW. Efficacy of varenicline for cigarette reduction before quitting in Japanese smokers: a subpopulation analysis of the reduce to quit trial. Clinical Therapeutics 2017;39(4):863-72.

\section{Etter 2002 \{published data only\}}

Dar R, Stronguin F, Etter JF. Assigned versus perceived placebo effects in nicotine replacement therapy for smoking reduction in Swiss smokers. Journal of Consulting and Clinical Psychology 2005;73(2):350-3.

Etter JF. Reduction of exposure to cigarette smoke: a randomized trial. 11th World Conference on Tobacco or Health; 2000 Aug 6-11; Chicago, USA. 2000:159.

Etter JF, Laszlo E. Postintervention effect of nicotine replacement therapy for smoking reduction: a randomized trial with a 5-year follow-up. Journal of Clinical Psychopharmacology 2007;27(2):151-5.

Etter JF, Laszlo E, Perneger TV. Postintervention effect of nicotine replacement therapy on smoking reduction in smokers who are unwilling to quit: randomized trial. Journal of Clinical Psychopharmacology 2004;24(2):174-9.

Etter JF, Laszlo E, Zellweger JP, Perrot C, Perneger TV. Nicotine replacement to reduce cigarette consumption in smokers who are unwilling to quit: a randomized trial. Society for Research on Nicotine and Tobacco 8th Annual Meeting; 2002 Feb 20-23; Savannah, USA. 2002:27. [PA6-3]

Etter JF, Perneger T, Zellweger JP. Smoking reduction with nicotine patches, gums, and inhalers; a randomised trial. Society for Research on Nicotine and Tobacco 3rd Europe Conference; 2001 Sept 19-22; Paris, France. 2001:20.

* EtterJF, Laszlo E, Zellweger JP, Perrot C, Perneger TV. Nicotine replacement to reduce cigarette consumption in smokers who are unwilling to quit: a randomized trial. Journal of Clinical Psychopharmacology 2002;22(5):487-95.

\section{Etter 2009 \{published data only\}}

Etter JF, Huguelet P, Perneger TV, Cornuz J. Nicotine gum treatment before smoking cessation: a randomized trial. Archives of Internal Medicine 2009;169(11):1028-34.

\section{Farley 2017 \{published data only\}}

* Farley A, Tearne S, Taskila T, Williams RH, MacAskill S, Etter JF, et al. A mixed methods feasibility study of nicotine-assisted smoking reduction programmes delivered by community pharmacists - The RedPharm study. BMC Public Health 2017;17(1):210-24

Taskila T, Macaskill S, Coleman T, Etter JF, Patel M, Clarke S, et al. A randomised trial of nicotine assisted reduction to stop in pharmacies - the RedPharm study. BMC Public Health 2012;12:182-94.

\section{Flaxman 1978 \{published data only\}}

Flaxman J. Gradual and abrupt quitting strategies and a successful self-control technique for smoking modification [thesis]. USA: Dissertation Abstracts International, 1975.

* Flaxman J. Quitting smoking now or later: Gradual, abrupt, immediate, and delayed quitting. Behavior Therapy 1978;9(2):260-70.

\section{Garcia 2000 \{published data only\}}

Garcia MP, Becona E. Evaluation of the amount of therapist contact in a smoking cessation program. Spanish Journal of Psychology 2000;3(1):28-36.

\section{Gariti 2004 \{published data only\}}

Gariti P, Alterman Al, Lynch KG, Kampman K, Whittingham T. Adding a nicotine blocking agent to cigarette tapering. Journal of Substance Abuse Treatment 2004;27(1):17-25.

\section{Gil Roales-Nieto 1992a \{published data only\}}

Gil Roales-Nieto J, Parra A. Efficiency of a self-control program for the treatment of smoking behaviour: Different effects in two strategies, one with an immediate quitting and other with a gradual quitting [Eficacia de un programa de autocontrol para el tratamiento del tabaquismo: Efectos diferenciales de dos estrategias de retirada de y reduccion]. Analisis y Modificacion de Conducta 1992;18(59):329-44.

\section{Glasgow 1978 \{published data only (unpublished sought but not used)\}}

Glasgow RE. Effects of a self-control manual, rapid smoking, and amount of therapist contact on smoking reduction. Journal of Consulting and Clinical Psychology 1978;46(6):1439-47.

Glasgow 1989 \{published data only\}

Glasgow RE, Morray K, Lichtenstein E. Controlled smoking versus abstinence as a treatment goal: The hopes and fears may be unfounded. Behavior Therapy 1989;20(1):77-91.

\section{Glasgow 2009a \{published data only\}}

Glasgow RE, Gaglio B, Estabrooks PA, Marcus AC, Ritzwoller DP, Smith TL, et al. Long-term results of a smoking reduction program. Medical Care 2009;47(1):115-20.

Gunther 1992 \{published data only\}

Gunther V, Gritsch S, Meise U. Smoking cessation - gradual or sudden stopping?. Drug and Alcohol Dependence 1992;29(3):231-6. 


\section{Hanson 2008 \{published data only\}}

Hanson K, Zylla E, Allen S, Avery G. Harm reduction: an intervention for adolescent smokers. Society for Research on Nicotine and Tobacco 12th Annual Meeting; 2006 Feb 15-18; Orlando, USA. 2006:4. [SYM3B]

* Hanson K, Zylla E, Allen S, Li Z, Hatsukami DK. Cigarette reduction: an intervention for adolescent smokers. Drug and Alcohol Dependence 2008;95(1-2):164-8.

NCT00158171. Effectiveness of various smoking cessation therapies in reducing smoking in adolescents - 1 . clinicaltrials.gov/ct2/show/NCT00158171 (first received 12 September 2005).

\section{Hao 2017 \{published data only\}}

Hao R, Zhou JP, Ni L, Li QY, Shi GC. Smoking abstinence rate and its associated factors between abrupt and gradual smoking cessation. Chinese Journal of Tuberculosis and Respiratory Diseases 2017;40(12):898-902.

\section{Hatsukami 2004 \{published data only\}}

* Hatsukami DK, Rennard S, Patel MK, Kotlyar M, Malcolm R, Nides MA, et al. Effects of sustained-release bupropion among persons interested in reducing but not quitting smoking. American Journal of Medicine 2004;116(3):151-7.

Rennard S, Hatsukami D, Malcolm RE, Patel MK, Jamerson BD, Dozier G. Zyban (bupropion HCL SR) vs placebo as an aid to smoking reduction among smokers unwilling and unable to quit smoking. Society for Research on Nicotine and Tobacco 7th Annual Meeting; 2001 Mar 20-23; Seattle, USA. 2001:117. [PO4 77]

\section{Haustein 2002 \{published data only\}}

* Haustein KO, Batra A. A double-blind, randomized, placebocontrolled multicenter trial of a nicotine chewing gum in smoking reduction. Erlangen, Germany: Pharmacia; 2002 November Report no. 980-CHC-9021-0031. [CHC-9021/ PMG0001]

Haustein KO, Batra A, Landfeldt B, Westin A. The effect of shortterm or long-term reduction on smoking cessation; results from a placebo controlled smoking reduction study with the nicotine gum. Nicotine \& Tobacco Research 2003;5(2):278.

\section{Ho 2018 \{published data only\}}

Ho KY, Li WH, Wang MP, Lam KK, Lam TH, Chan SS. Comparison of two approaches in achieving smoking abstinence among patients in an outpatient clinic: a Phase 2 randomized controlled trial. Patient Education and Counseling 2018;101(5):885-93.

\section{Hughes 2010 \{published data only\}}

Hughes JR, Callas PW. Is delaying a quit attempt associated with less success?. Nicotine \& Tobacco Research 2011;13(12):1228-32.

* Hughes JR, Solomon LJ, Livingston AE, Callas PW, Peters EN. A randomized, controlled trial of NRT-aided gradual vs. abrupt cessation in smokers actively trying to quit. Drug and Alcohol Dependence 2010;111(1-2):105-13.
NCT00297492. Gradual vs. abrupt cessation treatment for smoking. clinicaltrials.gov/ct2/show/NCT00297492 (first received 28 Feb 2006).

\section{Jerome 1992 \{published data only\}}

Jerome A, Perrone R, Kalfus G. Computer-assisted smoking treatment - a controlled evaluation and long-term follow-up. Journal of Advancement in Medicine 1992;5(1):29-41.

\section{Jerome 1999a \{published data only\}}

Jerome A, Behar A. Computerized, scheduled gradual reduction for smoking cessation: a randomized outcome study with 12month follow-up. Personal communication 1999.

* Jerome A, Behar A, Boyd NR. Computerized scheduled gradual reduction for smoking cessation: a randomized work-site outcome study with 12-month follow-up. Unpublished trial report.

\section{Joseph 2008 \{published data only\}}

Joseph AM, Bliss RL, Zhao F, Lando H. Predictors of smoking reduction without formal intervention. Nicotine \& Tobacco Research 2005;7(2):277-82.

* Joseph AM, Hecht SS, Murphy SE, Lando H, Carmella SG, Gross M, et al. Smoking reduction fails to improve clinical and biological markers of cardiac disease: a randomized controlled trial. Nicotine \& Tobacco Research 2008;10(3):471-81.

\section{Klemperer 2017 \{published data only\}}

Klemperer EM, Hughes JH. Reduction in cigarettes per day prospectively predicts quit attempts and cessation in smokers who are not ready to quit. Drug and Alcohol Dependence 2017;171:e107. [DOI: doi.org/10.1016/j.drugalcdep.2016.08.299]

Klemperer EM, Hughes JR, Callas PW, Solomon LJ. A mediation analysis of motivational, reduction, and usual care interventions for smokers who are not ready to quit. Nicotine \& Tobacco Research 2017;19(8):916-21. [DOI: 10.1093/ntr/ntx025]

Klemperer EM, Hughes JR, Callas PW, Solomon LJ. Working alliance and empathy as mediators of brief telephone counseling for cigarette smokers who are not ready to quit.. Psychology of Addictive Behaviors 2017;31(1):130. [DOI: 10.1037/ adb0000243]

* Klemperer EM, Hughes JR, Solomon LJ, Callas PW, Fingar JR. Motivational, reduction, and usual care interventions for smokers who are not ready to quit: A randomized controlled trial. Addiction 2017;112(1):146-55. [DOI: 10.1111/add.13594]

NCT01866722. Not quite ready to quit. clinicaltrials.gov/ct2/ show/NCT01866722 (first received 31 May 2013).

\section{Kralikova 2009 \{published data only\}}

Kralikova E, Kozak J, Rasmussen T, Cort N. Smoking reduction or cessation with nicotine gum or inhaler. Society for Research on Nicotine and Tobacco 7th Annual Meeting; 2001 Mar 20-23; Seattle, USA. 2001:118. [PO4 80]

Kralikova E, Kozak J, Rasmussen T, Cort N. The clinical benefits of NRT-supported smoking reduction. Nicotine \& Tobacco Research 2002;4(2):243. 
* Kralikova E, Kozak JT, Rasmussen T, Gustavsson G, Le Houezec J. Smoking cessation or reduction with nicotine replacement therapy: a placebo-controlled double blind trial with nicotine gum and inhaler. BMC Public Health 2009;9:433-40.

\section{Lindson-Hawley 2016b \{published data only\}}

ISRCTN22526020. Rapid reduction versus abrupt quitting for smokers who want to stop soon. www.isrctn.com/ ISRCTN22526020 (first received 14 October 2008).

Lindson N, Aveyard P, Ingram JT, Inglis J, Beach J, West R, et al. Rapid reduction versus abrupt quitting for smokers who want to stop soon: a randomised controlled non-inferiority trial. Trials 2009;10(69):1-14.

* Lindson-Hawley N, Banting M, West R, Michie S, Shinkins B, Aveyard P. Gradual versus abrupt smoking cessation: a randomized, controlled noninferiority trial. Annals of Internal Medicine 2016;164(9):585-92.

Lindson-Hawley N, Shinkins B, West R, Michie S, Aveyard P. Does cigarette reduction while using nicotine replacement therapy prior to a quit attempt predict abstinence following quit date? Addiction 2016;111(7):1275-82.

West R, Lindson-Hawley N, Aveyard P. Gradual versus abrupt smoking cessation. Annals of Internal Medicine 2016;165(10):742. [DOI: dx.doi.org/10.7326/L16-0401]

Wilson $\mathrm{H}$, Sherman $\mathrm{S}$. In adults, quitting smoking abruptly improved abstinence more than quitting gradually. Annals of Internal Medicine 2016;165(2):JC3. [10.7326/ ACPJC-2016-165-2-003 MISC1 - 201633/ DD: 20160809]

Malott 1984 \{published data only\}

Malott JM, Glasgow RE, O'Neill HK, Klesges RC. Co-worker social support in a worksite smoking control program. Journal of Applied Behavior Analysis 1984;17(4):485-95.

\section{NCT00158158 \{published data only\}}

NCT00158158. Effectiveness of reducing smoking in facilitating smoking cessation in adolescents - 2. clinicaltrials.gov/ct2/ show/NCT00158158 (first received 12 September 2005).

\section{Nicki 1984 \{published data only\}}

Nicki RM, Remington RE, MacDonald GA. Self-efficacy, nicotinefading/self-monitoring and cigarette-smoking behaviour. Behaviour Research and Therapy 1984;22(5):477-85.

\section{Ostroff 2014 \{published data only\}}

NCT00575718. Smoking cessation intervention for cancer patients. clinicaltrials.gov/ct2/show/NCT00575718 (first received 18 December 2007).

Ostroff J, Burkhalter J, Cinciripini P, Li Y, Shiyko M, Lam C, et al. Randomized trial of a pre-surgical, scheduled reduced smoking intervention for patients newly diagnosed with cancer. Psychooncology 2013;22(S2):74-75.

* Ostroff JS, Burkhalter JE, Cinciripini PM, Li Y, Shiyko MP, Lam CY, et al. Randomized trial of a presurgical scheduled reduced smoking intervention for patients newly diagnosed with cancer. Health Psychology 2014;33(7):737-47.
Sloan E, Lord-Bessen J, Hollan S, Li Y, Burkhalter J, Furberg A, et al. An analysis of treatment implementation fidelity as a possible explanation for null findings in a smoking cessation trial. Society for Research on Nicotine and Tobacco 18th Annual Meeting; 2012 Mar 13-16; Houston, USA. 2012:101. [POS3-32]

\section{Perez-Milena 2012 \{published data only\}}

Perez-Milena A, Navarreteguillen AB, Mesa-Gallardo MI, Martinez Perez R, Leal-Helmling FJ, Perez-Fuentes C. Efficiency of two motivational interventions for adolescent smokers (brief and intensive) conducted in High Schools [Eficiencia de dos intervenciones motivacionales para la deshabituación tabáquica en adolescentes (breve e intensiva) realizadas en Institutos de Educación Secundaria]. Adicciones 2012;24(3):191-9.

\section{Rennard 2006 \{published data only\}}

Rennard SI, Glover E, Leischow S, Daughton DM, Glover P, Muramoto M. Efficacy of nicotine inhaler in smoking reduction. Nicotine \& Tobacco Research 2002;4(3):380.

* Rennard SI, Glover ED, Leischow S, Daughton DM, Glover PN, Muramoto $\mathrm{M}$, et al. Efficacy of the nicotine inhaler in smoking reduction: A double-blind, randomized trial. Nicotine \& Tobacco Research 2006;8(4):555-64.

\section{Riley 2001 \{published data only\}}

Jerome A, Fiero PL, Behar A, Bensur A. Combining scheduled, gradual reduction with nicotine replacement. Society for Research on Nicotine and Tobacco Fifth Annual Meeting; 1999 Mar 5-7; San Diego, USA. 1999. [PO B50]

* Riley W, Behar A, Jerome A, Weil WS. Computerized scheduled gradual reduction of smoking prior to nicotine patch use: evidence of additive efficacy. Society for Research on Nicotine and Tobacco 7th Annual Meeting; 2001 Mar 20-23 Seattle, USA. 2001:74. [PO3 28]

\section{Riley 2005 \{published data only\}}

* Riley W. Computerized scheduling of nicotine nasal spray. USA: LifeSign; 2005. Study ID: R44DA013062.

Riley WT, Behar A, Shields R, Kelleher K, Vasiliadis M. Effects of a scheduled gradual crossover of cigarettes and nicotine nasal spray. Society for Research on Nicotine and Tobacco 11th Annual Meeting; 2005 Mar 20-23; Prague, Czech Republic. 2005.

Rohsenow 2016 \{published data only\}

Martin RA, Rohsenow D, Tidey JW, Colby S, Monti P. Treating smokers in substance treatment with contingent vouchers, nicotine replacement and brief advice adapted for sobriety settings. Drug and Alcohol Dependence 2015;156:e140-1.

* Rohsenow DJ, Martin RA, Tidey JW, Colby SM, Monti PM. Treating smokers in substance treatment with contingent vouchers, nicotine replacement and brief advice adapted for sobriety settings. Journal of Substance Abuse Treatment 2016;72:72-9.

\section{Ruther 2018 \{published data only\}}

Ruther T, Kiss A, Eberhardt K, Linhardt A, Kroger C, Pogarell O. Evaluation of the cognitive behavioral smoking reduction 
program "Smoke_less": a randomized controlled trial. European Archives of Psychiatry and Clinical Neuroscience 2018;268(3):269-77.

\section{Shiffman 2009 \{published data only\}}

Shiffman S, Dresler CM, Norton M, Strahs K. Using nicotine gum to assist quitting by gradual reduction. Society for Research on Nicotine and Tobacco 12th Annual Meeting; 2006 Feb 15-18, Orlando, USA. 2006:14. [SYM10D]

* Shiffman S, Ferguson SG, Strahs KR. Quitting by gradual smoking reduction using nicotine gum: a randomized controlled trial. American Journal of Preventive Medicine 2009;36(2):96-104.

\section{Wang 2017 \{published data only\}}

* Wang MP, Li WH, Cheung YT, Lam OB, Wu Y, Kwong AC, et al. Brief advice on smoking reduction versus abrupt quitting for smoking cessation in Chinese smokers: a cluster randomized controlled trial. Nicotine \& Tobacco Research 2017;20(1):67-72.

\section{Wennike 2003 \{published data only\}}

Wennike P, Danielsson T, Landfeldt B, Westin A, Tonnesen P. Smoking reduction promotes smoking cessation: results from a double blind, randomized, placebo-controlled trial of nicotine gum with 2-year follow-up. Addiction 2003;98(10):1395-402.

\section{Wu 2017 \{published data only\}}

Wu L, He Y, Jiang B, Zhang D, Tian H, Zuo F, et al. The effect of a very brief smoking-reduction intervention in smokers who have no intention to quit: study protocol for a randomized controlled trial. BMC Public Health 2015;15:418-23.

* Wu L, He Y, Jiang B, Zhang D, Tian H, Zuo F, et al. Very brief physician advice and supplemental proactive telephone calls to promote smoking reduction and cessation in Chinese male smokers with no intention to quit: a randomized trial. Addiction 2017;112(11):2032-40

\section{References to studies excluded from this review}

ACTRN12609000482268 \{published data only\}

ACTRN12609000482268. Randomised controlled trial of Zonnic and nicotine patch (ZAP) in smoking cessation [Randomised placebo-controlled trial of Zonnic and nicotine patch in heavy smokers for smoking cessation]. www.anzctr.org.au/Trial/ Registration/TrialReview.aspx?ACTRN=12609000482268 (First received 18 June 2009).

\section{ACTRN12617000905369 \{published data only\}}

ACTRN12617000905369. Feasibility of electronic nicotine devices for smoking cessation with alcohol and other drug treatment clients [The QuitENDs Study: a feasibility pilot study of electronic nicotine devices for smoking cessation with alcohol and other drug treatment clients]. www.anzctr.org.au/ Trial/Registration/TrialReview.aspx?id=373092 (First received 20 June 2017).

\section{Applegate 2004 \{published data only\}}

Applegate BW, Riley WT, Sowell A. A comparison of computerassisted scheduled gradual reduction vs. self help in unmotivated smokers. Society for Research on Nicotine and Tobacco 10th Annual Meeting; 2004 Feb 18-21; Phoenix, USA. 2004:41. [POS1-028]

\section{Armitage 2007 \{published data only\}}

Armitage CJ. Efficacy of a brief worksite intervention to reduce smoking: the roles of behavioral and implementation intentions. Journal of Occupational Health Psychology 2007;12(4):376-90. [DOI: 10.1037/1076-8998.12.4.376]

\section{Atwood 1975 \{published data only\}}

Atwood JT. The Influence of Differential Treatment Goals on the Success of a Behavior Modification Program for Smokers [thesis]. USA: Dissertation Abstracts International, 1975.

\section{Audrain McGovern 2011 \{published data only\}}

Audrain-McGovern J, Stevens S, Murray PJ, Kinsman S, Zuckoff A, Pletcher J, et al. The efficacy of motivational interviewing versus brief advice for adolescent smoking behavior change. Pediatrics 2011;128(1):e101-e111. [DOI: 10.1542/peds.2010-2174]

\section{Aveyard 2011 \{published data only\}}

Aveyard P, Begh R, Sheikh A, Amos A. Promoting smoking cessation through smoking reduction during Ramadan. Addiction 2011;106(8):1379-80. [DOI: https://dx.doi.org/10.1111/ j.1360-0443.2011.03432.x]

\section{Aveyard 2014 \{published data only\}}

Aveyard P, Lindson-Hawley N, Hastings G, De Andrade M. Should smokers be advised to cut down as well as quit?. BMJ 2014;348:g2787. [DOI: dx.doi.org/10.1136/bmj.g2787]

\section{Baker 2006 \{published data only\}}

* Baker A, Richmond R, Haile M, Lewin TJ, Carr VJ, Taylor RL, et al. A randomized controlled trial of a smoking cessation intervention among people with a psychotic disorder. American Journal of Psychiatry 2006;163(11):1934-42. [DOI: 10.1176/ appi.ajp.163.11.1934]

Baker A, Richmond R, Kay-Lambkin F, Lewin TJ, Carr VJ. Randomized controlled trial of a smoking cessation intervention among people with a psychotic disorder: 3-year follow-up. Society for Research on Nicotine and Tobacco 13th Annual Meeting; 2007 Feb 21-24, Austin, USA. 2007. [PA2-2]

Baker A, Richmond R, Lewin TJ, Kay-Lambkin F. Cigarette smoking and psychosis: naturalistic follow up 4 years after an intervention trial. Australian and New Zealand Journal of Psychiatry 2010;44(4):342-50. [DOI: 10.3109/00048670903489841]

Lan TH, Chiu HJ, Wu BJ, Hung TH, Hu TM. Readiness to quit and smoking reduction outcomes. American Journal of Psychiatry 2007;164(5):827-8

\section{Barbarin 1978 \{published data only\}}

Barbarin OA. Comparison of symbolic and overt aversion in the self-control of smoking. Journal of Consulting and Clinical Psychology 1978;46(6):1569-71. 
Batra 2003 \{published data only\}

Batra A, Haustein K-O, Friederich HM, Zschatsch I. Controlled smoking reduction - results of a placebo-controlled smoking reduction study with nicotine chewing gum [Kontrollierte rauchreduktion - ergebnisse aus einer placebokontrollierten rauchreduktionsstudie mit Nikotinkaugummi]. Verhaltenstherapie 2003;13(S1):5.

\section{Batra 2005 \{published data only\}}

* Batra A, Klingler K, Landfeldt B, Friederich HM, Westin A, Danielsson T. Smoking reduction treatment with 4-mg nicotine gum: a double-blind, randomized, placebo-controlled study. Clinical Pharmacology and Therapeutics 2005;78(6):689-96. [DOI: 10.1016/j.clpt.2005.08.019]

Landfeldt B, Batra A, Friederich HM, Klingler K, Westin A. Smoking reduction with a $4 \mathrm{mg}$ nicotine gum - final results from a placebo-controlled trial over 13 months. Society for Research on Nicotine and Tobacco 5th European Meeting; 2003 Nov 20-22; Padua, Italy. 2003.

\section{Beavers 1973 \{published data only\}}

Beavers ME. Smoking Control: a Comparison of Three Aversive Conditioning Treatments [thesis]. USA: Dissertation Abstracts International, 1973.

\section{Becona 1991 \{published data only\}}

Becona E, Gomez-Duran BJ. Reduction of cigarette intake at baseline and efficacy of a smoking cessation programme [Descenso del consumo de cigarrillos en la linea base y eficacia de un programa para dejar de fumar]. Revista Española de Drogodependencias 1991;16(4):277-84.

\section{Becona 1993 \{published data only\}}

Becona E, Garcia MP. Nicotine fading and smokeholding methods to smoking cessation. Psychological Reports 1993;73(3):779-86.

\section{Becona 1998 \{published data only\}}

Becona E, Vazquez FL. Efficacy of a behavioral multicomponent cessation program at five year follow-up. Research Communications in Biological Psychology \& Psychiatry 1998;23(1-2):9-17.

\section{Becona 2001 \{published data only\}}

Becona E, Vazquez FL. Comparison of the efficacy of a selfhelp smoking cessation by mail with a manual in one-time intervention or weekly intervention [Comparacion de la eficacia de un programa de autoayuda para dejar de fumar distriuido por correo en un manual en un unico envio o semanalmente en folletos]. Psiquis 2001;22(4):41-50.

\section{Becona Iglesias 1989 \{published data only\}}

Becona Iglesias E, Lista Rubianes MJ. Treatment of smokers with nicotine fading techniques. Psiquis Revista de Psiquiatria, Psicologia y Psicosomatica 1989;10(4):38-44.

\section{Berecz 1971 \{published data only\}}

Berecz JM. Reduction of smoking through self-administered aversion conditioning of imagined behavior. Annual Convention of the American Psychological Association. 1971:429-30.

\section{Berecz 1984 \{published data only\}}

Berecz, JM. Superiority of a low-contrast smoking cessation method. Addictive Behaviors 1984;9(3):273-8. [DOI: dx.doi.org/10.1016/0306-4603\%2884\%2990019-4]

Berger 2006 \{published data only\}

Berger, J. Smoking reduction and lung cancer risk. JAMA 2006;295(9):1001-2.

\section{Bernard 1972 \{published data only\}}

Bernard HS, Efran JS. Eliminating versus reducing smoking using pocket timers. Behaviour Research and Therapy 1972;10(4):399-401.

\section{Blalock 2001 \{published data only\}}

Blalock JA, Cinciripini PM, Hogan MC. Transdermal nicotine and gradual reduction for smoking cessation. Society for Research on Nicotine and Tobacco 7th Annual Meeting, 2001 Mar 21-23; Seattle, USA. 2001:74. [PO3 31]

Bloch 2010 \{published data only\}

Bloch B, Reshef A, Cohen T, Tafla A, Gathas S, Israel S, et al. Preliminary effects of bupropion and the promoter region (HTTLPR) serotonin transporter (SLC6A4) polymorphism on smoking behavior in schizophrenia. Psychiatry Research 2010;175(1-2):38-42. [DOI: 10.1016/j.psychres.2008.12.015]

Bolliger 2000b \{published data only\}

Bolliger CT. Practical experiences in smoking reduction and cessation. Addiction 2000;95(S1):19-24.

\section{Borland 1999 \{published data only\}}

Borland R, Owen N, Tooley G, Treijs I, Roberts L, Hill D. Promoting reduced smoking rates in the context of workplace smoking bans. American Journal of Health Promotion 1999;14(1):1-3. [DOI: 10.4278/0890-1171-14.1.1]

\section{Borland 2013 \{published data only\}}

Borland R, Balmford J, Swift E. Effects of timing of initiation and planning on smoking cessation outcomes: study protocol for a randomised controlled trial. BMC Public Health 2013;13:235. [DOI: 10.1186/1471-2458-13-235]

\section{Bowers 1987 \{published data only\}}

Bowers TG, Winett RA, Frederiksen LW. Nicotine fading, behavioral contracting, and extended treatment: effects on smoking cessation. Addictive Behaviors 1987;12(2):181-4.

\section{Bradford 1991 \{published data only\}}

Bradford TS. The Effects of Combining Behavioral Counselling with Nicotine Fading and Smoke Holding in Medically AtRisk Adult Smokers [thesis]. USA: Dissertation Abstracts International, 1991.

\section{Brown 1984 \{published data only\}}

Brown RA, Lichtenstein E, McIntyre KO, Harrington-Kostur J. Effects of nicotine fading and relapse prevention on smoking cessation. Journal of Consulting and Clinical Psychology 1984;52(2):307-8. [DOI: dx.doi.org/10.1037/0022-006X.52.2.307] 
Brue 2001 \{published data only\}

Brue V, Karlson K, Escamilla G. Utility of a computerized device in scheduled reduced smoking. Society for Research on Nicotine and Tobacco 7th Annual Meeting; 2001 Mar 21-23; Seattle, USA. 2001:106. [PO2 78)]

\section{Buceta 1989 \{published data only\}}

Buceta JM, Lopez A. Application of a behavioral-cognitive intervention program for smoking cessation. Revista Intercontinental de Psicologia y Educacion 1989;2(3):81-99.

\section{Burling 1982 \{published data only\}}

Burling TA, Stitzer ML, Bigelow GE, Russ NW. Techniques used by smokers during contingency motivated smoking reduction. Addictive Behaviors 1982;7(4):397-401.

\section{Burling 1989 \{published data only\}}

Burling TA, Marotta J, Gonzalez R, Moltzen JO, Eng AM, Schmidt GA, et al. Computerized smoking cessation program for the worksite: treatment outcome and feasibility. Journal of Consulting and Clinical Psychology 1989;57(5):619-22.

\section{Burling 1994 \{published data only\}}

Burling TA, Seidner AL, Gaither DE. A computer-directed program for smoking cessation treatment. Journal of Substance Abuse 1994;6(4):427-31.

\section{Burris 2014 \{published data only\}}

Burris JL, Carpenter MJ, Wahlquist AE, Cummings MK, Gray KM. Brief, instructional smokeless tobacco use among cigarette smokers who do not intend to quit: a pilot randomized clinical trial. Nicotine \& Tobacco Research 2014;16(4):397-405. [DOI: dx.doi.org/10.1093/ntr/ntt161]

\section{Cacciapaglia 2007 \{published data only\}}

Cacciapaglia HM, Kaplar ME, McConnell K, Schwetschenau HM, Carels RA. A stepped-care approach to smoking cessation and harm reduction. Annals of Behavioral Medicine 2007;33(S1):205.

\section{Caponnetto 2013 \{published data only\}}

* Caponnetto P, Campagna D, Cibella F, Morjaria JB, Caruso M, Russo $C$, et al. Efficiency and safety of an electronic cigarette (ECLAT) as tobacco cigarettes substitute: a prospective 12-month randomized control design study. PloS One 2013;8(6):e66317. [DOI: 10.1371/journal.pone.0066317]

NCT01194583. Randomised controlled trial investigating the efficacy and safety of an electronic nicotine delivery device (e-cigarette) without nicotine cartridges in smokers. clinicaltrials.gov/show/nct01194583 (first received 3 September 2010).

\section{Caponnetto 2014 \{published data only\}}

Caponnetto P, Polosa R, Auditore R, Minutolo G, Signorelli M, Maglia $\mathrm{M}$, et al. Smoking cessation and reduction in schizophrenia (SCARIS) with e-cigarette: study protocol for a randomized control trial. Trials 2014;15(1):88. [DOI: 10.1186/1745-6215-15-88]

\section{Cassidy 2018 \{published data only\}}

Cassidy RN, Jackson KM, Rohsenow DJ, Tidey JW, Tevyaw TO, Barnett NP, et al. Contingency management for college student smokers: the role of drinking as a moderator and mediator of smoking abstinence during treatment. Addictive Behaviors 2018;80:95-101. [DOI: 10.1016/j.addbeh.2018.01.017]

\section{Chambliss 1979 \{published data only\}}

Chambliss C, Murray EJ. Cognitive procedures for smoking reduction: symptom attribution versus efficacy attribution. Cognitive Therapy and Research 1979;3(1):91-5.

\section{Chen 2013 \{published data only\}}

Chen HK, Lan TH, Wu BJ. A double-blind randomized clinical trial of different doses of transdermal nicotine patch for smoking reduction and cessation in long-term hospitalized schizophrenic patients. European Archives of Psychiatry and Clinical Neuroscience 2012;263(1):75-82. [DOI: 10.1007/ s00406-012-0338-3]

\section{Cinciripini 1994 \{published data only\}}

Cinciripini PM, Lapitsky LG, Wallfisch A, Mace R, Nezami E, Van Vunakis $H$. An evaluation of a multicomponent treatment program involving scheduled smoking and relapse prevention procedures: initial findings. Addictive Behaviors 1994;19(1):13-22.

\section{Colby 2005 \{published data only\}}

Colby SM, Monti PM, O'Leary Tevyaw T, Barnett NP, Spirito A, Rohsenow DJ, et al. Brief motivational intervention for adolescent smokers in medical settings. Addictive Behaviors 2005;30(5):865-74.

\section{Colletti 1978 \{published data only\}}

Colletti G. The Relative Efficacy of Participant Modeling, Participant Observer, and Self-monitoring Procedures as Maintenance Strategies Following a Positive Behaviorally Based Treatment for Smoking Reduction [thesis]. USA: Dissertation Abstracts International, 1978.

\section{Colletti 1979 \{published data only\}}

Colletti G, Stern L. Two-year follow-up of a nonaversive treatment for cigarette smoking. Journal of Consulting and Clinical Psychology 1980;48(2):292-3.

\section{Colletti 1980 \{published data only\}}

Colletti G, Supnick JA. Continued therapist contact as a maintenance strategy for smoking reduction.. Journal of Consulting and Clinical Psychology 1980;48(5):665-7. [DOI: dx.doi.org/10.1037/0022-006X.48.5.665]

Colletti 1982 \{published data only\}

Colletti G, Supnick JA, Rizzo AA. Long-term follow-up (3-4 years) of treatment for smoking reduction. Addictive Behaviors 1982;7(4):429-33.

\section{Corty 1984 \{published data only\}}

Corty E, McFall RM. Response prevention in the treatment of cigarette smoking.. Addictive Behaviors 1984;9(4):405-8. 


\section{Cropsey 2008 \{published data only\}}

Cropsey KL, Jackson DO, Hale GJ, Carpenter MJ, Stitzer ML. Impact of self-initiated pre-quit smoking reduction on cessation rates: results of a clinical trial of smoking cessation among female prisoners. Addictive Behaviors 2011;36(1-2):73-8.

\section{Cropsey 2015 \{published data only\}}

Cropsey KL, Clark CB, Hendricks PS. Varenicline for smoking reduction prior to cessation. JAMA 2015;313(22):2284-5. [DOI: dx.doi.org/10.1001/jama.2015.4972]

\section{Crosbie 1972 \{published data only\}}

Crosbie PV, Petroni FA, Stitt BG. The dynamics of "corrective" groups. Journal of Health and Social Behavior 1972;13(3):294-302. [DOI: dx.doi.org/10.2307/2136766]

D'Ruiz 2017 \{published data only\}

D'Ruiz CD, O'Connell G, Graff DW, Yan XS. Measurement of cardiovascular and pulmonary function endpoints and other physiological effects following partial or complete substitution of cigarettes with electronic cigarettes in adult smokers. Regulatory Toxicology and Pharmacology 2017;87:36-53. [DOI: 10.1016/j.yrtph.2017.05.002]

Darity 1997 \{published data only\}

Darity WA, Chen TT, Tuthill RW, Buchanan DR, Winder AE, Stanek $\mathrm{E}$, et al. A multi-city community based smoking research intervention project in the African-American population. International Quarterly of Community Health Education 1997;17(2):117-30. [DOI: dx.doi.org/10.2190/CEXY-WG7C-GL3EA2BP]

\section{Daughton 1994 \{published data only\}}

Daughton DM, Millatmal T, Susman JL, Belenky S, Sitorius M, Floreani AA, et al. Abrupt abstinence is critical to smoking cessation success in a family practice setting. Journal of Smoking-Related Disorders 1994;5(S1):319.

\section{Delahunt 1977 \{published data only\}}

Delahunt JG. Effectiveness Assessment of Modified Treatments for the Elimination of Smoking [thesis]. USA: Dissertation Abstracts International, 1977.

\section{Dlack 1999 \{published data only\}}

Dlack GW, Meador-Woodruff JH. Nicotine replacement and smoking reduction in smokers with schizophrenia. Schizophrenia Research 1999;1-3:276.

\section{Dogris 1998 \{published data only\}}

Dogris NJ. The Effect of a Prerecorded Hypnosis CD and Behavioral Fading on Smoking Cessation and Locus of Control [thesis]. USA: Dissertation Abstracts International, 1998.

\section{Ebbert 2010 \{published data only\}}

Ebbert JO, Edmonds A, Luo X, Jensen J, Hatsukami DK. Smokeless tobacco reduction with the nicotine lozenge and behavioral intervention. Nicotine \& Tobacco Research 2010;12(8):823-7. [DOI: dx.doi.org/10.1093/ntr/ntq088]

\section{Ehrsam 1991 \{published data only\}}

Ehrsam RE, Buhler A, Muller P, Mauli D, Schumacher PM, Howald $\mathrm{H}$, et al. Weaning of young smokers using a transdermal nicotine patch. Schweizerische Rundschau fur Medizin Praxis (Revue Suisse de Medecine Praxis) 1991;80(7):145-50.

Elliott 1978 \{published data only\}

Elliott CH, Denney DR. A multiple component treatment approach to smoking reduction. Journal of Consulting and Clinical Psychology 1978;46(6):1330-9. [DOI: dx.doi.org/10.1037//0022-006X.46.6.1330]

Emmons 1988 \{published data only\}

Emmons KM, Emont SL, Collins RL, Weidner G. Relapse prevention versus broad spectrum treatment for smoking cessation: a comparison of efficacy. Journal of Substance Abuse 1988;1(1):79-89.

\section{Etringer 1984 \{published data only\}}

Etringer BD, Gregory VR, Lando HA. Influence of group cohesion on the behavioral treatment of smoking. Journal of Consulting and Clinical Psychology 1984;52(6):1080-6. [DOI: dx.doi.org/10.1037/0022-006X.52.6.1080]

Etter 2011 \{published data only\}

Etter JF. Comparing abrupt and gradual smoking cessation: a randomized trial. Drug and Alcohol Dependence 2011;118(2-3):360-5. [DOI: 10.1016/j.drugalcdep.2011.04.016]

Euler 1973 \{published data only\} Euler VHA. Reduction of cigarette smoking through selfmonitoring [Die reduktion des zigarettenrauchens durch selbst-monitoring]. Zeitschrift fur Klinische Psychologie und Psychotherapie 1973;21(3):271-82.

\section{Evins 2001 \{published data only\}}

Evins AE, Cather C, Rigotti NA, Freudenreich O, Henderson DC, Olm Shipman CM, et al. Two-year follow-up of a smoking cessation trial in patients with schizophrenia: increased rates of smoking cessation and reduction. Journal of Clinical Psychiatry 2004;65(3):307-11.

* Evins AE, Mays VK, Rigotti NA, Tisdale T, Cather C, Goff DC. A pilot trial of bupropion added to cognitive behavioral therapy for smoking cessation in schizophrenia. Nicotine \& Tobacco Research 2001;3(4):397-403. [DOI: 10.1080/14622200110073920]

Evins AE, Mays VK, Rigotti NA, Tisdale T, Daigle A, Goff DC. Reduction In tobacco use in schizophrenia with bupropion SR and Cognitive Behavioral Therapy. Society for Research on Nicotine and Tobacco 6th Annual Meeting; 2000 Feb 18-20; Arlington, USA. 2000.

\section{Evins 2007 \{published data only\}}

Evins AE, Cather C, Culhane M, Birnbaum AS, Horowitz J, Hsieh E, et al. A placebo-controlled study of bupropion SR added to high dose nicotine replacement therapy for smoking cessation or reduction in schizophrenia. Society for Research on Nicotine and Tobacco 12th Annual Meeting; 2006 Feb 15-18; Orlando, USA. 2006:101. [POS2-104] 
* Evins AE, Cather C, Culhane MA, Birnbaum A, Horowitz J, Hsieh E, et al. A 12-week double-blind, placebo-controlled study of bupropion SR added to high-dose dual nicotine replacement therapy for smoking cessation or reduction in schizophrenia. Journal of Clinical Psychopharmacology 2007;27(4):380-6. [DOI: 10.1097/01.jcp.0b013e3180ca86fa]

NCT00307203. Safety and effectiveness of sustained release bupropion in treating individuals with schizophrenia who smoke. clinicaltrials.gov/show/NCT00307203 (first received 27 March 2006).

\section{Fagerström 2002a \{published data only\}}

Fagerström K. Smoking reduction in the management of COPD. Monaldi Archives for Chest Disease (Archivio Monaldi per le malattie del torace) 2002;57(5-6):281-4.

\section{Farkas 2001 \{published data only\}}

Farkas AJ, Pierce JP, Zhu SH. Home smoking restrictions: a brief cessation intervention for callers to the California Smoker's Helpline who are not ready to quit. Society for Research on Nicotine and Tobacco 7th Annual Meeting; 2001 March 21-23; Seattle, USA. 2001:109. [PO4 48]

\section{Fatemi 2005 \{published data only\}}

Fatemi SH, Stary JM, Hatsukami DK, Murphy SE. A doubleblind placebo-controlled cross over trial of bupropion in smoking reduction in schizophrenia. Schizophrenia Research 2005;76(2-3):353-6. [DOI: 10.1016/j.schres.2005.02.021]

\section{Fatemi 2013 \{published data only\}}

Fatemi SH, Yousefi MK, Kneeland RE, Liesch SB, Folsom TD, Thuras PD. Antismoking and potential antipsychotic effects of varenicline in subjects with schizophrenia or schizoaffective disorder: a double-blind placebo and bupropion-controlled study. Schizophrenia Research 2013;146(1-3):376-8. [DOI: dx.doi.org/10.1016/j.schres.2013.02.015]

\section{Feryo 2009 \{published data only\}}

Feryo MR. Self-Directed Smoking Cessation Program [thesis]. USA: Dissertation Abstracts International, 2009.

\section{Filia 2010 \{published data only\}}

Filia S, Baker A, Richmond R, Kay-Lambkin F, Castle D, Williams J, et al. Randomised controlled trial of a healthy lifestyles intervention to reduce cardiovascular disease (CVD) risk among smokers with psychosis: interim results. Australian and New Zealand Journal of Psychiatry 2010;44(S1):A31-2. [DOI: dx.doi.org/10.3109/00048674.2010.507064]

\section{Forgays 1987 \{published data only\}}

Forgays DG. Flotation rest as a smoking intervention. Addictive Behaviors 1987;12(1):85-90. [DOI: dx.doi.org/10.1016/0306-4603\%2887\%2990014-1]

\section{Foxx 1979 \{published data only\}}

${ }^{*}$ Foxx RM, Brown RA. Nicotine fading and self-monitoring for cigarette abstinence or controlled smoking. Journal of Applied Behavior Analysis 1979;12(1):111-25.
Foxx RM, Brown RM, Katz I. Nicotine fading and self-monitoring for cigarette abstinence or controlled smoking: a two and onehalf year follow-up. Behavior Therapist 1981;4(2):21-3.

\section{Foxx 1983 \{published data only\}}

Foxx RM, Axelroth E. Nicotine fading, self-monitoring and cigarette fading to produce cigarette abstinence or controlled smoking. Behaviour Research and Therapy 1983;21(1):17-27. [DOI: dx.doi.org/10.1016/0005-7967\%2883\%2990122-5]

\section{Franklin 2009 \{published data only\}}

Franklin TR, Harper D, Kampman K, Kildea-McCrea S, Jens W, Lynch KG, et al. The GABA B agonist baclofen reduces cigarette consumption in a preliminary double-blind placebocontrolled smoking reduction study. Drug and Alcohol Dependence 2009;103(1-2):30-6. [DOI: hdx.doi.org/10.1016/ j.drugalcdep.2009.02.014]

\section{Frederiksen 1976 \{published data only\}}

Frederiksen LW, Peterson Gl. Controlled smoking: development and maintenance. Addictive Behaviors 1976;1:193-6.

\section{Fuhrer 1972 \{published data only\}}

Fuhrer RE. The Effects of Covert Sensitization with Relaxation Induction, Covert Sensitization Without Relaxation Induction, and Attention-Placebo on the Reduction of Cigarette Smoking [thesis]. USA: Dissertation Abstracts International, 1972.

\section{Galizia 1990 \{published data only\}}

Galizia VJ. A Test of a Compliance Model for Prediction of Smoking Reduction in Cardiopulmonary Patients [thesis]. USA: Dissertation Abstracts International, 1990.

\section{Gariti 2002 \{published data only\}}

Gariti P, Alterman A, Mulvaney F, Mechanic K, Dhopesh V, Yu E, et al. Nicotine intervention during detoxification and treatment for other substance use. American Journal of Drug and Alcohol Abuse 2002;28(4):671-9.

\section{Gelkopf 2012 \{published data only\}}

Gelkopf M, Noam S, Rudinski D, Lerner A, Behrbalk P, Bleich A, et al. Nonmedication smoking reduction program for inpatients with chronic schizophrenia: a randomized control design study. Journal of Nervous and Mental Disease 2012;200(2):142-6. [DOI: 10.1097/NMD.0b013e3182438e92]

\section{Gil Roales-Nieto 1992b \{published data only\}}

Gil Roales-Nieto J. Nicotine fading and external control fading as a procedure for controlling smoking. Psicothema 1992;4(2):397-412.

\section{Glasgow 1983 \{published data only\}}

Glasgow RE, Klesges RC, Godding PR, Gegelman R. Controlled smoking, with or without carbon monoxide feedback, as an alternative for chronic smokers. Behavior Therapy 1983;14(3):386-97.

\section{Glasgow 1984 \{published data only\}}

* Glasgow RE, Klesges RC, Godding PR, Vasey MW, O'Neill HK. Evaluation of a worksite-controlled smoking program. Journal of Consulting and Clinical Psychology 1984;52(1):137-8. 
Glasgow RE, Klesges RC, Klesges LM. Long-term effects of a controlled smoking program: a $21 / 2$ year follow-up. Behavior Therapy 1985;16(3):303. [DOI: dx.doi.org/10.1016/ S0005-7894\%2885\%2980018-6]

\section{Glasgow 1986 \{published data only\}}

Glasgow RE, Klesges RC, O'Neill HK. Programming social support for smoking modification: an extension and replication. Addictive Behaviors 1986;11(4):453-7.

\section{Glasgow 2009b \{published data only\}}

* Glasgow RE, Estabrooks PA, Marcus AC, Smith TL, Gaglio B, Levinson $\mathrm{AH}$, et al. Evaluating initial reach and robustness of a practical randomized trial of smoking reduction. Health Psychology 2008;27(6):780-8. [DOI: 10.1037/0278-6133.27.6.780]

Levinson AH, Glasgow RE, Gaglio B, Smith TL, Cahoon J, Marcus AC. Tailored behavioral support for smoking reduction: development and pilot results of an innovative intervention. Health Education Research 2008;23(2):335-46. [DOI: 10.1093/ her/cym046]

\section{Gonzalez 1991 \{published data only\}}

Gonzalez RC. Cognitive Assessment in Computerized Nicotine Fading [thesis]. USA: Dissertation Abstracts International, 1991.

\section{Gradl 2009 \{published data only\}}

Gradl S, Kroeger C, Floeter S, Piontek D. Effectiveness of a modern smoking cessation programme based on international guidelines. Verhaltenstherapie und Verhaltensmedizin 2009;30(2):169-85

\section{Graff 1966 \{published data only\}}

Graff H, Hammet VO, Bash N, Fackler G, Yanovski A, Goldman A. Results of four antismoking methods. Pennsylvania Medical Journal 1966;69:86-9.

\section{Gulliver 2008 \{published data only\}}

Gulliver SB, Kamholz BW. Helstrom AW, Morissette SB, Kahler CW. A preliminary evaluation of adjuncts to motivational interviewing for psychiatrically complex smokers. Journal of Dual Diagnosis 2008;4(4):394-413. [DOI: 10.1080/15504260802086149]

\section{Gutmann 1967 \{published data only\}}

Gutmann M, Marston A. Problems of S's motivation in a behavioral program for reduction of cigarette smoking. Psychological Reports 1967;20(3 (2)):1107-14. [DOI: dx.doi.org/10.2466/pr0.1967.20.3c.1107]

\section{Gylys 2000 \{published data only\}}

Gylys JA. Smoking Cessation in a Public Health Primary Care Clinic: A Minimal Assistance Scheduled Smoking Approach [thesis]. USA: Dissertation Abstracts International:, 2000.

\section{Hamilton 1998 \{published data only\}}

Hamilton BD. Nicotine Fading Versus Abrupt Quitting: Testing Educational and Behavioral Smoking Cessation Treatment Strategies [thesis]. USA: Dissertation Abstracts International, 1998.

\section{Hatsukami 1988 \{published data only\}}

Hatsukami DK, Dahlgren L, Zimmerman R, Hughes JR. Symptoms of tobacco withdrawal from total cigarette cessation versus partial cigarette reduction. Psychopharmacology 1988;94(2):242-7.

\section{Hatsukami 2005 \{published data only\}}

* Hatsukami DK, Kotlyar M, Allen S, Jensen J, Li S, Le C, et al. Effects of cigarette reduction on cardiovascular risk factors and subjective measures. Chest 2005;128(4):2528-37. [DOI: 10.1378/ chest.128.4.2528]

Kotlyar M, Jensen J, Li Shelby, Hatsukami DK. Effect of smoking reduction on cardiovascular biomarkers and subjective measures. Nicotine \& Tobacco Research 2004;6(4):719.

Hawk 2015 \{published data only\}

Hawk LW Jr, Ashare RL, Rhodes JD, Oliver JA, Cummings KM, Mahoney MC. Does extended pre quit bupropion aid in extinguishing smoking behavior?. Nicotine \& Tobacco Research 2015;17(11):1377-84. [DOI: 10.1093/ntr/ntu347]

Hilleman 1994 \{published data only\}

Hilleman DE, Mohiuddin SM, Delcore MG. Comparison of fixed-dose transdermal nicotine, tapered-dose transdermal nicotine, and buspirone in smoking cessation. Journal of Clinical Pharmacology 1994;34(3):222-4.

Hills 1982 \{published data only\} Hills AA. Target-setting self-control for smoking. Psychological Reports 1982;50(1):68-70. [DOI: dx.doi.org/10.2466/ pro.1982.50.1.68]

\section{Hovell 2009 \{published data only\}}

Hovell MF, Zakarian JM, Matt GE, Liles S, Jones JA, Hofstetter CR, et al. Counseling to reduce children's secondhand smoke exposure and help parents quit smoking: a controlled trial. Nicotine \& Tobacco Research 2009;11(12):1383-94. [DOI: 10.1093/ntr/ntp148]

Hughes 1991 \{published data only\} Hughes JR, Gust SW, Keenan R, Fenwick JW, Skoog K, Higgins ST. Long-term use of nicotine vs placebo gum. Archives of Internal Medicine 1991;151(10):1993-8.

\section{Hughes 2004b \{published data only\}}

Hughes J, Lindgren P, Connett J, Nides M, Lung Health Study. Smoking reduction in the Lung Health Study. Nicotine \& Tobacco Research 2004;6(2):275-80. [DOI: $10.1080 / 14622200410001676297]$

\section{Hughes 2007 \{published data only\}}

Hughes JR. Smokers who choose to quit gradually versus abruptly. Addiction 2007;102(8):1326-7.

\section{Hughes 2011 \{published data only\} \\ Hughes JR, Rennard SI, Fingar JR, Talbot SK, Callas PW, Fagerström KO. Efficacy of varenicline to prompt quit attempts in smokers not currently trying to quit: a randomized placebo-controlled trial. Nicotine \& Tobacco Research 2011;13(10):955-64. [10.1093/ntr/ntr103 MISC1 - DC:20110928]}


Hughes 2016 \{published data only\}

Hughes JR, Klemperer EM. Gradual versus abrupt smoking cessation. Annals of Internal Medicine 2016;165(10):741. [DOI: dx.doi.org/10.7326/L16-0402]

\section{Hurt 1990 \{published data only\}}

Hurt RD, Lauger GG, Offord KP, Kottke TE, Dale LC. Nicotinereplacement therapy with use of a transdermal nicotine patch a randomized double-blind placebo-controlled trial. Mayo Clinic Proceedings 1990;65(12):1529-37.

\section{ISRCTN13288677 \{published data only\}}

ISRCTN13288677. Can electronic cigarettes and nicotine replacement treatment help reduce smoking in smokers who struggle to quit?. www.isrctn.com/ISRCTN13288677 (first received 17 March 2017).

\section{ISRCTN13837944 \{published data only\}}

ISRCTN13837944. Improving behavioural support for reducing smoking among those who want to cut down [An exploratory trial to evaluate the effects of a physical activity intervention as a smoking cessation induction and cessation aid among the 'hard to reach']. www.isrctn.com/ISRCTN13837944 (first received 5 July 2010).

\section{ISRCTN64013828 \{published data only\}}

ISRCTN64013828. The non-concealed placebo: a randomized trial on smoking cessation [An open-label randomized interventional study to assess the effect of placebo smoking cessation patches versus no treatment on nicotine dependence scores of adult Filipino smokers]. www.isrctn.com/ ISRCTN64013828 (first received 24 February 2016).

\section{Jacobs 1971 \{published data only\}}

Jacobs MA, Spilken AZ, Norman MM, Wohlberg GW, Knapp PH. Interaction of personality and treatment conditions associated with success in a smoking control program. Psychosomatic Medicine 1971;33(6):545-56.

Jeon 2016 \{published data only\}

* Jeon DW, Shim JC, Kong BG, Moon JJ, Seo YS, Kim SJ, et al. Adjunctive varenicline treatment for smoking reduction in patients with schizophrenia: a randomized doubleblind placebo-controlled trial. Schizophrenia Research 2016;176(2-3):206-11. [DOI: 10.1016/j.schres.2016.08.016]

\section{Jerome 1999b \{published data only\}}

Jerome A, Fiero PL. Computerized scheduling of nicotine gum use (personal communication. email to: Lindson N 1999.

\section{Joksic 2011 \{published data only\}}

Joksic G, Spasojevic-Tisma V, Antic R, Nilsson R, Rutqvist LE. Randomized, placebo-controlled, double-blind trial of Swedish snus for smoking reduction and cessation. Harm Reduction Journal 2011;8:25. [DOI: dx.doi.org/10.1186/1477-7517-8-25]

\section{Joseph 2005 \{published data only\}}

Joseph A, Hecht S, Murphy S, Gross M, Lando H, Bliss R, et al. A randomized controlled trial of smoking reduction in heart disease patients. Nicotine \& Tobacco Research 2005;7(4):687.

\section{Karam-Hage 2014 \{published data only\}}

Karam-Hage M, Robinson JD, Lodhi A, Brower KJ. BupropionSR for smoking reduction and cessation in alcohol-dependent outpatients: a naturalistic, open-label study. Current Clinical Pharmacology 2014;9(2):123-9.

Karoly 1975 \{published data only\}

Karoly P, Doyle WW. Effects of outcome expectancy and timing of self monitoring on cigarette smoking. Journal of Clinical Psychology 1975;31(2):351-5.

\section{KCT0001277 \{published data only\}}

KCT0001277. Effect of an electronic cigarette for smoking reduction and cessation in Korean male smokers: a randomized, controlled study. apps.who.int/trialsearch/Trial3.aspx? trialid=KCT0001277 (first received 14 November 2014).

Kelly 2010 \{published data only\}

Kelly AC, Zuroff DC, Foa CL, Gilbert P. Who benefits from training in self-compassionate self-regulation? A study of smoking reduction. Journal of Social and Clinical Psychology 2010;29(7):727-55. [DOI: dx.doi.org/10.1521/jscp.2010.29.7.727]

\section{Keutzer 1968 \{published data only\}}

Keutzer CS. Behavior modification of smoking: the experimental investigation of diverse techniques. Behaviour Research and Therapy 1968;6(2):137-57. [DOI: dx.doi.org/10.1016/0005-7967\%2868\%2990001-6]

\section{Klein 2010 \{published data only\}}

Klein G, Ulbricht S, Haug S, Gross B, Rumpf H-J, John U, et al. Effects of practitioner-delivered brief counseling and computergenerated tailored letters on cigarettes per day among smokers who do not quit - a quasi-randomized controlled trial. Drug and Alcohol Dependence 2010;112(1-2):81-9. [DOI: dx.doi.org/10.1016/j.drugalcdep.2010.05.016]

\section{Klemperer 2016 \{published data only\}}

Klemperer EM, Hughes JR. After precessation nicotine replacement therapy, abrupt cessation increases abstinence more than gradual cessation in smokers ready to quit. EvidenceBased Medicine 2016;21(5):174. [DOI: dx.doi.org/10.1136/ ebmed-2016-110489]

\section{Lamb 2004 \{published data only\}}

Lamb RJ, Morral AR, Kirby KC, Iguchi MY, Galbicka G. Shaping smoking cessation using percentile schedules. Drug and Alcohol Dependence 2004;76(3):247-59. [DOI: 10.1016/ j.drugalcdep.2004.05.008]

\section{Lamb 2007 \{published data only\}}

Lamb RJ, Morral AR, Kirby KC, Javors MA, Galbicka G, Iguchi M. Contingencies for change in complacent smokers. Experimental and Clinical Psychopharmacology 2007;15(3):245-55. [DOI: 10.1037/1064-1297.15.3.245]

\section{Lamb 2010 \{published data only\}}

Lamb RJ, Kirby KC, Morral AR, Galbicka G, Iguchi MY. Shaping smoking cessation in hard-to-treat smokers. Journal of Consulting and Clinical Psychology 2010;78(1):62-71. [DOI: 10.1037/a0018323] 
Lan 2007 \{published data only\}

Lan TH, Chiu HJ, Wu BJ, Hung TH, Hu TM. Readiness to quit and smoking reduction outcomes.. American Journal of Psychiatry 2007;164(5):827-8.

\section{Lando 1985 \{published data only\}}

Lando HA, McGovern PG. Nicotine fading as a nonaversive alternative in a broad-spectrum treatment for eliminating smoking. Addictive Behaviors 1985;10(2):153-61.

\section{Larson 1999 \{published data only\}}

Larson ME. Behavioral Substitutes and Smoking Cessation: Do They Make Quitting Easier? [thesis]. USA: Dissertation Abstracts International, 1999.

\section{Leischow 2004 \{published data only\}}

Leischow SJ, Djordjevic MV. Smoking reduction and tobaccorelated cancers: the more things change, the more they stay the same. Journal of the National Cancer Institute 2004;96(2):86-7.

\section{Levinson 1971 \{published data only\}}

Levinson BL, Shapiro D, Schwartz GE, Tursky B. Smoking elimination by gradual reduction. Behavior Therapy 1971;2(4):477-87. [DOI: dx.doi.org/10.1016/ S0005-7894\%2871\%2980094-1]

\section{Levinson 2008 \{published data only\}}

Levinson AH, Glasgow RE, Gaglio B, Smith TL, Cahoon J, Marcus AC. Tailored behavioral support for smoking reduction: development and pilot results of an innovative intervention. Health Education Research 2008;23(2):335-46.

\section{Lichtenstein 1967 \{published data only\}}

Lichtenstein E, Poussaint AF, Bergman SH, Jurney T, Shapiro R. A further report on the effects of the physician's treating of smoking by placebo. Diseases of the Nervous System 1967;28(11):754-5.

\section{Lillington 1998 \{published data only\}}

Lillington GA, Sachs DP. Preoperative smoking reduction: all or nothing at all?. Chest 1998;113(4):856-8.

\section{Macgregor 1996 \{published data only\}}

Macgregor ID. Efficacy of dental health advice as an aid to reducing cigarette smoking. British Dental Journal 1996;180(8):292-6.

\section{Marston 1971 \{published data only\}}

Marston AR, McFall RM. Comparison of behavior modification approaches to smoking reduction. Journal of Consulting and Clinical Psychology 1971;36(2):153-62. [DOI: dx.doi.org/10.1037/ h0030736]

\section{Meredith 2011 \{published data only\}}

Meredith SE, Grabinski MJ, Dallery J. Internet-based group contingency management to promote abstinence from cigarette smoking: a feasibility study. Drug and Alcohol Dependence 2011;118(1):23-30.
Mihaltan 2007 \{published data only\}

Mihaltan F. Smoking reduction - an alternative solution? [Reducerea fumatului - o solutie alternativa pentru alte oferte?]. Pneumologia 2007;56(4):218-20.

\section{Morris 2011 \{published data only\}}

Morris CD, Waxmonsky JA, May MG, Tinkelman DG, Dickinson M, Giese AA. Smoking reduction for persons with mental illnesses: 6-month results from community-based interventions. Community Mental Health Journal 2011;47(6):694-702. [DOI: 10.1007/s10597-011-9411-z]

Mueller 2012 \{published data only\} Mueller S, Petitjean S, Degen B, Wiesbeck GA. Smoking cessation in alcohol-dependent patients: Results from a randomized, controlled trial. Alcohol and Alcoholism 2011;46:i7.

\section{Muramoto 1999 \{published data only\}}

Muramoto ML, Leischow S, Cook G, Castellini SM, Strayer L. Effectiveness of over-the-counter transdermal nicotine for smoking reduction. Nicotine \& Tobacco Research 1999;1(2):107-7.

\section{Murray 1981 \{published data only\}}

Murray RG, Hobbs SA. Effects of self-reinforcement and self-punishment in smoking reduction: implications for broad-spectrum behavioral approaches. Addictive Behaviors 1981;6(1):63-7.

\section{Myette 1993 \{published data only\}}

Myette TL. Maritime Command's new smoking-reduction strategy unprecedented among world's navies. Canadian Medical Association journal (Journal de l'Association Medicale Canadienne) 1993;149(2):206-8.

Myles 1994 \{published data only\} Myles PS. Smoking reduction and tape suggestion. Anaesthesia 1994;49(10):917-8.

\section{Møller 2002 \{published data only\}}

Møller AM, Villebro N, Pedersen T, Tønnesen H. Effect of preoperative smoking intervention on postoperative complications: a randomised clinical trial. Lancet 2002;359:114-7. [DOI: 10.1016/S0140-6736(02)07369-5]

\section{NCT01772641 \{published data only\}}

NCT01772641. A smoking intervention study using scheduled gradual reduction with varenicline to help with cessation. clinicaltrials.gov/show/nct01772641 (first received 21 January 2013).

NCT01982110 \{published data only\}

NCT01982110. A mindfulness based application for smoking cessation. clinicaltrials.gov/show/nct01982110 (first received 13 November 2013).

\section{NCT03128554 \{published data only\}}

NCT03128554. Smoking reduction: testing a novel approach to cessation in primary care practice settings. clinicaltrials.gov/ show/NCT03128554 (first received 25 April 2017). 
Nentwig 1978 \{published data only\}

Nentwig CG. Attribution of cause and long-term effects of the modification of smoking behavior. Behavior Analysis and Modification 1978;2(4):285-95.

\section{Newman 1982 \{published data only\}}

Newman A, Bloom R. Smoking reduction: a comparison of the effectiveness of rapid smoking and increasing delay training. Addictive Behaviors 1982;7(1):93-6.

\section{Noonan 2018 \{published data only\}}

Noonan D, Silva S, Njuru J, Bishop T, Fish LJ, Simmons LA, et al. Feasibility of a text-based smoking cessation intervention in rural older adults. Health Education Research 2018;33(1):81-8. [DOI: 10.1093/her/cyx080]

\section{NTR5113 \{published data only\}}

NTR5113. Blended smoking cessation treatment - evaluation of effectiveness of a blended face-to-face and web-based smoking cessation treatment versus treatment as usual (randomised controlled trial) - (LiveSmokefree-Study). www.trialregister.nl/ trial/4975 (first received 24 March 2015).

\section{O'Brien 2015 \{published data only\}}

O'Brien B, Knight-West O, Walker N, Parag V, Bullen C. Ecigarettes versus NRT for smoking reduction or cessation in people with mental illness: secondary analysis of data from the ASCEND trial. Tobacco Induced Diseases 2015;13(1):5. [DOI: dx.doi.org/10.1186/s12971-015-0030-2]

\section{O'Connor 1998 \{published data only\}}

O'Connor K, Langlois R. Changes in confidence and craving during smoking reduction. Clinical Psychology \& Psychotherapy 1998;5:145-54.

\section{Olbrich 2008 \{published data only\}}

Olbrich R, Trager S, Nikitopoulos J, Mann K, Diehl A. Smoking reduction during inpatient alcohol detoxification: a controlled clinical pilot trial [Rauchreduktion bei alkoholkranken im rahmen einer stationaren alkohol-entzugsbehandlung: eine kontrollierte klinische pilotstudie]. Fortschritte der NeurologiePsychiatrie 2008;76(5):272-7. [DOI: dx.doi.org/10.1055/ s-2008-1038168]

\section{Orleans 1991 \{published data only\}}

Orleans CT, Schoenbach VJ, Wagner EH, Quade D, Salmon MA, Pearson DC, et al. Self-help quit smoking interventions: effects of self-help materials, social support instructions, and telephone counseling. Journal of Consulting and Clinical Psychology 1991;59(3):439-48.

\section{Patten 1996 \{published data only\}}

Patten CA. Effectiveness of Cognitive-Behavioral Depression Therapy as an Adjunct to Smoking Cessation Treatment for Recovering Alcoholics [thesis]. USA: Dissertation Abstracts International, 1996.

\section{Pisinger 2005 \{published data only\}}

Pisinger C. Smoking cessation and smoking reduction in a general population. The Inter99 study. A randomised population-based intervention study. Nordic Journal of Psychiatry 2006;60(1):67.

* Pisinger C, Vestbo J, Borch-Johnsen K, Jørgensen T. Smoking reduction intervention in a large population-based study. The Inter99 study. Preventive Medicine 2005;40(1):112-8. [DOI: 10.1016/j.ypmed.2004.05.014]

Relinger 1977 \{published data only\}

Relinger H, Bornstein PH, Bugge ID, Carmody TP, Zohn CJ. Utilization of adverse rapid smoking in groups: efficacy of treatment and maintenance procedures. Journal of Consulting and Clinical Psychology 1977;45(2):245-9. [DOI: dx.doi.org/10.1037/0022-006X.45.2.245]

Rennard 1993 \{published data only\}

Rennard SI, Daughton DM. Smoking reduction and biological markers of response. Monaldi Archives for Chest Disease (Archivio Monaldi per le Malattie del Torace) 1993;48(5):580-2.

\section{Rennard 2010 \{published data only\}}

Rennard S, Hughes JP, Kralikova E, Raupach T, Arteaga C. Randomized, placebo-controlled trial of varenicline for smoking cessation with flexible quit dates. European Respiratory Society Annual Congress; 2010 Sept 18-22; Barcelona, Spain. 2010.

Riggs 2001 \{published data only\}

Riggs RL, Hughes JR, Pillitteri JL. Two behavioral treatments for smoking reduction: a pilot study. Nicotine \& Tobacco Research 2001;3(1):71-6

Riley 2002 \{published data only\}

Riley W, Jerome A, Behar A, Weil J. Computer and manual self-help behavioral strategies for smoking reduction: Initial feasibility and one-year follow-up. Nicotine and Tobacco Research 2002;4(S2):183-8.

Ritchie 1991 \{published data only\}

Ritchie PJ. Abstinence Versus Controlled Smoking as Goals in Behavioural and Educational Smoking Treatment Programmes [thesis]. USA: Dissertation Abstracts International, 1991.

Robey 2015 \{published data only\}

Robey RB, Block CA, O'Rourke DJ. Varenicline for smoking reduction prior to cessation. JAMA 2015;313(22):2285. [DOI: dx.doi.org/10.1001/jama.2015.4978]

Rose 2010 \{published data only\}

Rose JE, Behm FM, Drgon T, Johnson C, Uhl GR. Personalized smoking cessation: interactions between nicotine dose, dependence and quit-success genotype score. Molecular Medicine 2010;16(7-8):247-53. [DOI: dx.doi.org/10.2119/ molmed.2009.00159]

\section{Rovina 2003 \{published data only\}}

Rovina N, Gratziou C, Nikoloutsou I, Athanassa Z, Francis K, Roussos C. Short or prolonged treatment with bupropion $\mathrm{HCL}$ in smoking cessation therapy. European Respiratory Journal 2003;22(S45):165. 
Royce 1995 \{published data only\}

Royce JM, Ashford A, Resnicow K, Freeman HP, Caesar AA, Orlandi MA. Physician- and nurse-assisted smoking cessation in Harlem. Journal of the National Medical Association 1995;87(4):291-300.

\section{Rutter 1990 \{published data only\}}

Rutter S. Cigarette-smoking reduction in university students. Psychological Reports 1990;66(1):186.

\section{Salk 1976 \{published data only\}}

Salk GC. A Comparison of Two Smoking Reduction Treatments Under Conditions Designed to be Interfering or Not Interfering With the Smoking Habit [thesis]. USA: Dissertation Abstracts International, 1976.

\section{Schiller 2012 \{published data only\}}

Hatsukami D, Anderson A, Jensen J. Immediate vs. gradual reduction toward cessation in smokeless tobacco user. Society for Research on Nicotine \& Tobacco 17th Annual Meeting; 2011 Feb 16-19; Toronto, Canada. 2011. [SYM 12A]

* Schiller KR, Luo X, Anderson AJ, Jensen JA, Allen SS, Hatsukami DK. Comparing an immediate cessation versus reduction approach to smokeless tobacco cessation. Nicotine \& Tobacco Research 2012;14(8):902-9. [DOI: 10.1093/ntr/ntr302]

\section{Schinke 1978 \{published data only\}}

Schinke SP, Blythe BJ, Doueck HJ. Reducing cigarette smoking: evaluation of a multifaceted interventive program. Behavioral Engineering 1978;4(4):107-12.

\section{Schleicher 2010 \{published data only\}}

Schleicher HE. Evaluation of a Cognitive-Behavioral Mood Management Intervention for Depressed College Smokers [thesis]. USA: Dissertation Abstracts International, 2010.

\section{Schuurmans 2016 \{published data only\}}

Schuurmans MM. Gradual versus abrupt smoking cessation. Annals of Internal Medicine 2016;165(10):741-2. [DOI: dx.doi.org/10.7326/L16-0403]

\section{Schwartz 1967 \{published data only\}}

Schwartz JL, Dubitzky M. Clinical reduction of smoking: a California study. California's Health 1967;24(6):78-84.

\section{Scott 1986 \{published data only\}}

Scott RR, Prue DM, Denier CA, King AC. Worksite smoking intervention with nursing professionals: long-term outcome and relapse assessment. Journal of Consulting and Clinical Psychology 1986;54(6):809-13. [DOI: dx.doi.org/10.1037/0022-006X.54.6.809]

\section{Severson 2000 \{published data only\}}

Severson HH, Akers L, Andrews JA, Lichtenstein E, Jerome A. Evaluating two self-help interventions for smokeless tobacco cessation. Addictive Behaviors 2000;25(3):465-70.

\section{Sipich 1974 \{published data only\}}

Sipich JF, Russell RK, Tobias LL. A comparison of covert sensitization and "nonspecific" treatment in the modification of smoking behavior. Journal of Behavior Therapy and Experimental Psychiatry 1974;5(2):201-3. [DOI: dx.doi.org/10.1016/0005-7916\%2874\%2990115-3]

Smith 2017 \{published data only\}

Smith DK, Miller DE, Mounsey A. "Cold turkey" works best for smoking cessation.. Journal of Family Practice 2017;66(3):174-6.

Spanos 1993 \{published data only\}

Spanos NP, Sims A, De Faye B, Mondoux TJ, Gabora NJ. A comparison of hypnotic and nonhypnotic treatments for smoking. Imagination, Cognition and Personality 1992;12(1):23-43

\section{Srivastava 2007 \{published data only\}}

Srivastava GN. Seven steps of gradual cessation of smoking - an example from India. Vojnosanitetski Pregled 2007;64(6):405-8.

\section{Stein 2002 \{published data only\}}

Stein JH, Bushara M, Bushara K, McBride PE, Jorenby DE, Fiore MC. Smoking cessation, but not smoking reduction, reduces plasma homocysteine levels. Clinical Cardiology 2002;25(1):23-6.

\section{Steinberg 2018 \{published data only\}}

Steinberg ML, Lu SE, Williams JM. Varenicline for smoking reduction in smokers not yet ready to quit: a double-blind, proof-of-concept randomized clinical trial. Addictive Behaviors 2018;84:20-6. [DOI: 10.1016/j.addbeh.2018.03.026]

\section{Stitzer 1985 \{published data only\}}

Stitzer ML, Bigelow GE. Contingent reinforcement for reduced breath carbon monoxide levels: target-specific effects on cigarette smoking. Addictive Behaviors 1985;10(4):345-9.

\section{Suedfeld 1974 \{published data only\}}

Suedfeld P, Ikard FF. Use of sensory deprivation in facilitating the reduction of cigarette smoking. Journal of Consulting and Clinical Psychology 1974;42(6):888-95.

\section{Sutherland 1975 \{published data only\}}

Sutherland A, Amit Z, Golden M, Roseberger Z. Comparison of three behavioral techniques in the modification of smoking behavior. Journal of Consulting and Clinical Psychology 1975;43(4):443-7.

\section{Templer 1969 \{published data only\}}

Templer DI. Aversive conditioning through hypnosis to reduce smoking. Association for Advancement of Behavior Therapy Newsletter 1969;4(3):9.

\section{Thompson 2016 \{published data only\}}

Taylor AH, Thompson TP, Greaves CJ, Taylor RS, Green C, Warren $\mathrm{FC}$, et al. A pilot randomised trial to assess the methods and procedures for evaluating the clinical effectiveness and cost-effectiveness of Exercise Assisted Reduction then Stop (EARS) among disadvantaged smokers. Health Technology Assessment 2014;18(4):1-324. [DOI: 10.3310/hta18040]

* Thompson TP, Greaves CJ, Ayres R, Aveyard P, Warren FC, Byng R, et al. An exploratory analysis of the smoking and 
physical activity outcomes from a pilot randomized controlled trial of an exercise assisted reduction to stop smoking Intervention in disadvantaged groups. Nicotine \& Tobacco Research 2016;18(3):289-97. [DOI: 10.1093/ntr/ntv099]

Thompson TP, Greaves CJ, Ayres R, Aveyard P, Warren FC, Byng R, et al. Lessons learned from recruiting socioeconomically disadvantaged smokers into a pilot randomized controlled trial to explore the role of Exercise Assisted Reduction then Stop (EARS) smoking. Trials 2015;16(1):1. [DOI: 10.1186/1745-6215-16-1]

\section{Thorndike 2006 \{published data only\}}

Thorndike FP, Friedman-Wheeler DG, Haaga DA. Effect of cognitive behavior therapy on smokers' compensatory coping skills. Addictive Behaviors 2006;31(9):1705-10.

\section{Thuerauf 2007 \{published data only\}}

Thuerauf N, Lunkenheimer J, Lunkenheimer B, Sperling W, Bleich S, Schlabeck M, et al. Memantine fails to facilitate partial cigarette deprivation in smokers--no role of Memantine in the treatment of nicotine dependency?. Journal of Neural Transmission 2007;114(3):351-7.

Tidey 2002 \{published data only\}

Tidey JW, O'Neill SC, Higgins ST. Contingent monetary reinforcement of smoking reductions, with and without Experimental and Clinical Psychopharmacology 2002;10(3):241-7.

\section{Tseng 2016 \{published data only\}}

* Tseng TY, Ostroff JS, Campo A, Gerard M, Kirchner T, Rotrosen J, Shelley D. A randomized trial comparing the effect of nicotine versus placebo electronic cigarettes on smoking reduction among young adult smokers. Nicotine \& Tobacco Research 2016;18(10):1937-43. [DOI: 10.1093/ntr/ntw017]

\section{Tønnesen 2005 \{published data only\}}

Tønnesen P, Pisinger C, Hvidberg S, Wennike P, Bremann L, Westin A, et al. Effects of smoking cessation and reduction in asthmatics. Nicotine \& Tobacco Research 2005;7(1):139-48. [DOI: 10.1080/14622200412331328411]

\section{Weidberg 2018 \{published data only\}} Perez A, Secades-Villa R. In-treatment cigarette demand among treatment-seeking smokers with depressive symptoms. Addictive Behaviors 2018;82:35-43. [DOI: 10.1016/ j.addbeh.2018.02.022]

\section{Wetter 2006 \{published data only\}}

Wetter D, Cinciripini PM. Palmtop computer-delivered treatments for smoking cessation. Society for Research on Nicotine and Tobacco 12th Annual Meeting; 2006 Feb 15-18; Orlando, USA. 2006. [SYM4C]

\section{Wewers 2009 \{published data only\}}

Wewers ME, Ferketich AK, Harness J, Paskett ED. Effectiveness of a nurse-managed, lay-led tobacco cessation intervention among Ohio Appalachian women. Cancer Epidemiology, transdermal nicotine, in outpatients with schizophrenia.

Weidberg S, Vallejo-Seco G, Gonzalez-Roz A, Garcia-

Biomarkers \& Prevention 2009;18(12):3451-8. [DOI: 10.1158/1055-9965.EPI-09-0952]

White 2011 \{published data only\}

White TJ. Effectiveness of a Brief Behavioral Smoking Cessation Intervention in a Residential Substance Abuse Treatment Center [thesis]. USA: Dissertation Abstracts International, 2011.

\section{Wiseman 1998 \{published data only\}}

Wiseman EJ. Nicotine replacement therapy and smoking reduction as an interim goal. JAMA 1998;279(3):194-5.

\section{References to studies awaiting assessment}

\section{Cinciripini 2001 \{published data only\}}

Cinciripini PM, Blalock JA, Wetter DW, Genac D, Seyed A, Seay S. Scheduled smoking and transdermal nicotine replacement. Society for Research on Nicotine and Tobacco 7th Annual Meeting; 2001 Mar 20-23 Seattle, USA. 2001:32.

\section{Cooper 1990 \{published data only\}}

Cooper T, Clayton RR. Behaviour modification and nicotine reduction therapy with heavy smokers: Comparison of four different dosing strategies. 7th World Conference on Tobacco and Health; 1990 Apr 1-5; Perth, Australia. 1990.

\section{Engeln 1969 \{published data only\}}

Engeln RG. A comparison of desensitization and aversive conditioning as treatment methods to reduce cigarette smoking [thesis]. USA: Dissertation Abstracts International, 1969.

\section{Gardner 1971 \{published data only\}}

Gardner RM. A Test of Coverant Control Therapy to Reduce Cigarette Smoking: A Comparative Study of the Effectiveness of Two Different Strategies with a Direct Test of the Effectiveness of Contingency Management [thesis]. USA: Dissertation Abstracts International, 1971.

\section{Palmer 1983 \{published data only\}}

Palmer RD. A Multiple Stage Treatment for Smoking Reduction [thesis]. USA: Dissertation Abstracts International, 1983.

\section{Rennard 1994 \{published data only\}}

Rennard SI, Daughton DM, Thompson AB, Floreani AA, Romberger DJ, Millatmal T. The effects of nicotine replacement therapy on cigarette smoking reduction. Annual Meeting of the American Thoracic Association; 1994; Boston, USA. 1994.

\section{Weis 1974 \{published data only\}}

Weis JI. An Experimental Examination of Cautela's Covert Sensitization as a Smoking Reduction Technique [thesis]. USA: Dissertation Abstracts International, 1974.

\section{References to ongoing studies}

Hagens 2017 \{published data only\}
${ }^{*}$ Hagens P, Pieterse M, Van der Valk P, Van der Palen J.
Effectiveness of intensive smoking reduction counselling plus
combination nicotine replacement therapy in promoting long-
term abstinence in patients with chronic obstructive pulmonary

Smoking reduction interventions for smoking cessation (Review) 
disease not ready to quit smoking: protocol of the REDUQ trial. Contemporary Clinical Trials Communications 2017;8:248-57.

NTR2227. Effects of a reduction-to-quit smoking programme in patients with COPD: the REDUQ study [A randomised controlled trial with 18 months follow-up in COPD patients comparing a smoking reduction programme with a self-help intervention, on reduction followed by sustained cessation]. www.trialregister.nl/trial/2110 (first received 2 February 2010).

NCT02124187 \{published data only\}

NCT02124187. Smoking cessation and reduction in depression. clinicaltrials.gov/ct2/show/NCT02124187 (first received 28 April 2014).

\section{NCT02354872 \{published data only\}}

NCT02354872. Motivation project: testing intervention components for the smoker who is unwilling to quit. clinicaltrials.gov/ct2/show/NCT02354872 (first received 3 February 2015).

\section{NCT02370147 \{published data only\}}

NCT02370147. Very brief smoking reduction intervention. clinicaltrials.gov/ct2/show/NCT02370147 (first received 24 February 2015).

\section{NCT02515500 \{published data only\}}

NCT02515500. Cigarette reduction using the quitbit digital lighter and mobile application for smoking cessation: a randomized controlled trial. clinicaltrials.gov/ct2/show/ NCT02515500 (first received 4 August 2015).

\section{NCT02564315 \{published data only\}}

NCT02564315. The long-term quitting (smoking cessation) study. clinicaltrials.gov/ct2/show/NCT02564315 (first received 30 September 2015).

\section{NCT02894957 \{published data only\}}

NCT02894957. Varenicline for "gradual" vs. "abrupt" smoking cessation in low-motivated COPD smokers. clinicaltrials.gov/ ct2/show/NCT02894957 (first received 9 September 2016).

\section{NCT02905656 \{published data only\}}

NCT02905656. Strategies to promote cessation in smokers who are not ready to quit. clinicaltrials.gov/ct2/show/NCT02905656 (first received 19 September 2016).

\section{Additional references}

\section{Bandura 1977}

Bandura A. Self efficacy: toward a unifying theory of behavioral change. Psychological Review 1977;84(2):191-215.

\section{Bouton 1991}

Bouton ME, Swartzentruber D. Sources of relapse after extinction in Pavlovian and instrumental learning. Clinical Psychology Review 1991;11(2):123-40. [DOI: doi.org/10.1016/0272-7358(91)90091-8]

\section{Cahill 2016}

Cahill K, Lindson-Hawley N, Thomas KH, Fanshawe TR, Lancaster T. Nicotine receptor partial agonists for smoking cessation. Cochrane Database of Systematic Reviews 2016, Issue 5. [DOI: 10.1002/14651858.CD006103.pub7]

\section{CDC 2017}

Centers for Disease Control and Prevention. Quitting smoking among adults-United States, 2000-2015. Morbidity and Mortality Weekly Report 2017; Vol. 65, issue 52:1457-64.

\section{Chaiton 2016}

Chaiton M, Diemert L, Cohen JE, Bondy SJ, Selby P, Philipneri A, et al. Estimating the number of quit attempts it takes to quit smoking successfully in a longitudinal cohort of smokers. BMJ Open 2016;6(6):1-9. [DOI: 10.1136/bmjopen-2016-011045]

\section{Chamberlain 2017}

Chamberlain C, O'Mara-Eves A, Porter J, Coleman T, Perlen SM, Thomas J, et al. Psychosocial interventions for supporting women to stop smoking in pregnancy. Cochrane Database of Systematic Reviews 2017, Issue 2. [DOI: 10.1002/14651858.CD001055.pub5]

\section{Cheong 2007}

Cheong Y, Yong H, Borland R. Does how you quit affect success? A comparison between abrupt and gradual methods using data from the international tobacco control policy evaluation study. Nicotine \& Tobacco Research 2007;9(8):801-10.

\section{Cohen 1983}

Cohen S, Hobermann HM. Positive events and social supports as buffers of life change stress. Journal of Applied Social Psychology 1983;13:99-125.

\section{Denning 2002}

Denning P. Harm reduction psychotherapy: an innovative alternative to classical addictions theory. American Clinical Laboratory 2002;21(4):16-8.

\section{Etter 2005}

Etter JF. A self-administered questionnaire to measure cigarette withdrawal symptoms: the Cigarette Withdrawal Scale. Nicotine \& Tobacco Research 2005;7(1):47-57.

\section{Fagerström 2002b}

Fagerström KO, Hughes JR. Nicotine concentrations with concurrent use of cigarettes and nicotine replacement: A review. Nicotine \& Tobacco Research 2002;4((Suppl 2)):S73-9.

\section{Fagerström 2005}

Fagerström KO. Can reduced smoking be a way for smokers not interested in quitting to actually quit?. Respiration 2005;72(2):216-20. [DOI: 10.1159/000084057]

\section{Fiore 2008}

Fiore MC, Jaen CR, Baker TB, Bailey WC, Benowitz NL, Curry SJ, et al. Treating tobacco use and dependence: 2008 update. Available at www.ncbi.nlm.nih.gov/books/NBK63952/ 2008. 


\section{Garnett 2019}

Garnett C, Shahab L, Raupach T, West R, Brown J. Understanding the Association Between Spontaneous Quit Attempts and Improved Smoking Cessation Success Rates: A Population Survey in England With 6-Month Follow-up. Nicotine \& Tobacco Research 2019;epub before print. [DOI: 10.1093/ntr] ntz115]

\section{GBD 2015 Risk Factors Collaborators 2016}

GBD 2015 Risk Factors Collaborators. Global, regional, and national comparative risk assessment of 79 behavioural, environmental and occupational, and metabolic risks or clusters of risks, 1990-2015: a systematic analysis for the Global Burden of Disease Study 2015. Lancet 2016;388:1659-724. [DOI: doi.org/10.1016/S0140-6736(16)31679-8]

\section{Hajek 1989}

Hajek P. Withdrawal-oriented therapy for smokers. British Journal of Addiction 1989;84:591-8.

\section{Hartmann-Boyce 2018}

Hartmann-Boyce J, Chepkin SC, Ye W, Bullen C, Lancaster T. Nicotine replacement therapy versus control for smoking cessation. Cochrane Database of Systematic Reviews 2018, Issue 5. [DOI: 10.1002/14651858.CD000146.pub5]

\section{Higgins 2011}

Higgins JP, Green S, editor(s). Cochrane Handbook for Systematic Reviews of Interventions Version 5.1.0 (updated March 2011). The Cochrane Collaboration, 2011. Available from www.cochrane-handbook.org. Cochrane Collaboration.

\section{Higgins 2017}

Higgins JP, Altman DG, Sterne JA, editor(s). Chapter 8: Assessing risk of bias in included studies. In: Higgins JP, Churchill R, Chandler J, Cumpston MS, editor(s), Cochrane Handbook for Systematic Reviews of Interventions version 5.2.0 (updated June 2017), The Cochrane Collaboration, 2017. Available from www.training.cochrane.org/handbook.

\section{Hughes 1986}

Hughes JR, Hatsukami D. Signs and symptoms of tobacco withdrawal. Archives of General Psychiatry 1986;43:289-94.

\section{Hughes 2004a}

Hughes JR, Keely J, Naud S. Shape of the relapse curve and long-term abstinence among untreated smokers. Addiction 2004;99(1):29-38. [DOI: doi.org/10.1111/ j.1360-0443.2004.00540.x]

\section{Hughes 2006}

Hughes JR, Carpenter MJ. Does smoking reduction increase future cessation and decrease disease risk? A qualitative review.. Nicotine \& Tobacco Research 2006;8(6):739-49. [DOI: $10.1080 / 14622200600789726]$

\section{Hughes 2014}

Hughes JR, Stead LF, Hartmann-Boyce J, Cahill K, Lancaster T. Antidepressants for smoking cessation. Cochrane Database of Systematic Reviews 2014, Issue 1. [DOI: 10.1002/14651858.CD000031.pub4]

\section{Jha 2011}

Jha P. Avoidable deaths from smoking: a global perspective. Public Health Reviews 2011;33(2):569-600.

\section{Lancaster 2017}

Lancaster T, Stead LF. Individual behavioural counselling for smoking cessation. Cochrane Database of Systematic Reviews 2017, Issue 3. [DOI: 10.1002/14651858.CD001292.pub3]

\section{Lindson 2012}

Lindson N. Smoking reduction and nicotine preloading: new approaches to cessation? [PhD thesis]. Available at www.etheses.bham.ac.uk/3245/1/Lindson12PhD.pdf 2012.

\section{Lindson 2019}

Lindson N, Chepkin SC, Ye W, Fanshawe TR, Bullen C, HartmannBoyce J. Different doses, durations and modes of delivery of nicotine replacement therapy for smoking cessation. Cochrane Database of Systematic Reviews 2019, Issue 4. [DOI: 10.1002/14651858.CD013308]

\section{Lindson-Hawley 2016a}

Lindson-Hawley N, Hartmann-Boyce J, Fanshawe TR, Begh R, Farley A, Lancaster T. Interventions to reduce harm from continued tobacco use. Cochrane Database of Systematic Reviews 2016, Issue 10. [DOI: 10.1002/14651858.CD005231.pub3]

\section{Livingstone-Banks 2019}

Livingstone-Banks J, Ordóñez-Mena JM, Hartmann-Boyce J. Print-based self-help interventions for smoking cessation. Cochrane Database of Systematic Reviews 2019, Issue 1. [DOI: 10.1002/14651858.CD001118.pub4]

\section{McRobbie 2006}

McRobbie H, Whittaker R, Bullen C. Using nicotine replacement therapy to assist in reducing cigarette consumption before quitting: another strategy for smoking cessation?. Disease Management and Health Outcomes 2006;4(6):335-40.

\section{Moore 2009}

Moore D, Aveyard P, Connock M, Wang D, Fry-Smith A, Barton P. Effectiveness and safety of nicotine replacement therapy assisted reduction to stop smoking: systematic review and meta-analysis. BMJ 2009;338:b1024. [DOI: 10.1136/bmj.b1024]

\section{NICE 2018}

National Institute for Health and Care Excellence. Stop smoking interventions and services. NICE guideline [NG92]. www.nice.org.uk/guidance/ng92 2018 (Accessed 21 May 2018).

\section{Peters 2007}

Peters EN, Hughes JR, Callas PW, Solomon LJ. Goals indicate motivation to quit smoking. Addiction 2007;102(7):1158-63. [DOI: doi.org/10.1111/j.1360-0443.2007.01870.x]

\section{Schünemann 2017}

Schünemann HJ, Oxman AD, Higgins JP, Vist GE, Glasziou P, Akl E, et al on behalf of the Cochrane GRADEing Methods Group and the Cochrane Statistical Methods Group. Chapter 11: Completing 'Summary of findings' tables and grading the 
confidence in or quality of the evidence. In: Higgins JP, Churchill R, Chandler J, Cumpston MS, editor(s), Cochrane Handbook for Systematic Reviews of Interventions version 5.2.0 (updated June 2017). The Cochrane Collaboration, 2017. Available from www.training.cochrane.org/handbook.

\section{Shiffman 2007}

Shiffman S, Hughes JR, Ferguson SG, Pillitteri JL, Gitchell JG, Burton SL. Smokers' interest in using nicotine replacement to aid smoking reduction. Nicotine \& Tobacco Research 2007;9(11):1177-82.

\section{Skinner 1953}

Skinner BF. Science and Human Behavior. Oxford: MacMillan, 1953.

\section{Stead 2017}

Stead LF, Carroll AJ, Lancaster T. Group behaviour therapy programmes for smoking cessation. Cochrane Database of Systematic Reviews 2017, Issue 3. [DOI: 10.1002/14651858.CD001007.pub3]

\section{Wang 2008}

Wang D, Connock M, Barton P, Fry-Smith A, Aveyard P, Moore D. 'Cut down to quit' with nicotine replacement therapies in smoking cessation: a systematic review of effectiveness and economic analysis. Health Technology Assessment 2008;12(2):1-135.

\section{West 2005}

West R, Hajek P, Stead L, Stapleton J. Outcome criteria in smoking cessation trials: proposal for a common standard. Addiction 2005;100(3):299-303. [DOI: 10.1111/ j.1360-0443.2004.00995.x]

\section{West 2006}

West 2006. Smoking and smoking cessation in England: 2006. 66.102.1.104/scholar?hl=en\&lr=\&client=firefoxa\&q=cache:UDuwOCSGZssJ:www.aspsilverbackwebsites.co.uk/
smokinginengland/Ref/paper4.pdf $+\% 22$ smoking+and+smoking +cessation+in+england\%22+2006+west 2006

\section{West 2012}

West R, Brown J. Smoking and smoking cessation in England 2011: Findings from the smoking toolkit study. http:// www.minerva-ebm.be/Resource/Get/10033 (accessed 12 September 2019).

\section{Wu 2015}

Wu L, Sun S, He Y, Zeng J. Effect of smoking reduction therapy on smoking cessation for smokers without an intention to quit: an updated systematic review and meta-analysis of randomized controlled trials. International Journal of Environmental Research and Public Health 2015;12(9):10235-53. [DOI: 10.3390/ ijerph120910235]

\section{References to other published versions of this review}

\section{Lindson 2009}

Lindson N, Aveyard P, Hughes JR. Reduction versus abrupt cessation in smokers who want to quit. (Protocol). Cochrane Database of Systematic Reviews 2009, Issue 4. [DOI: 10.1002/14651858.CD008033]

\section{Lindson 2010}

Lindson N, Aveyard P, Hughes JR. Reduction versus abrupt cessation in smokers who want to quit. Cochrane Database of Systematic Reviews 2010, Issue 3. [DOI: 10.1002/14651858.CD008033.pub2]

\section{Lindson-Hawley 2012}

Lindson-Hawley N, Aveyard P, Hughes JR. Reduction versus abrupt cessation in smokers who want to quit. Cochrane Database of Systematic Reviews 2012, Issue 11. [DOI: 10.1002/14651858.CD008033.pub3]

* Indicates the major publication for the study

\section{CHARACTERISTICS OF STUDIES}

Characteristics of included studies [ordered by study ID]

Blevins 2016

\begin{tabular}{ll} 
Methods & Study design: RCT \\
& Location: USA \\
& Setting: research fitness facility and telephone \\
& Recruitment: newspaper and radio advertisements \\
\hline Participants & Specialist population?: sedentary lifestyle (exercising < 60 minutes per week during previous 6 months) \\
& $\begin{array}{l}\text { Participant characteristics: } 40 / 61(66 \%) \text { female; average age: } 47.3 \text { y; average cig/day: } 20 ; \text { nicotine de- } \\
\text { pendence: FTND } 5.7\end{array}$
\end{tabular}


Blevins 2016 (Continued)

Preference for quitting abruptly versus gradually: not reported

Interventions

All participants received counselling. Sessions were designed to prepare participants for their quit date using a number of strategies. One of these was practiced reduction of $1-2$ cigarettes/day prior to quitting. Other strategies were identifying high-risk situations for cigarette use, developing and using coping strategies, setting incremental goals, and relapse prevention

Comparator: Health education: health education sessions on topics such as oral health, heart disease, cancer, sleep hygiene, and secondhand smoke, as they related to the effects of smoking, given in lectures, handouts, in-group exercises, and Internet resources

Modality of support: face-to-face and telephone

Overall contact time: 14 h 40 mins (12-h health education; 2 h 40 mins smoking cessation)

Number of sessions: 20 (12 health education counselling, 8 smoking cessation)

Pharmacotherapy: 8 weeks nicotine patch post-quit

Quit date set?: yes

Intervention: Aerobic exercise: aerobic exercise sessions supervised by an exercise physiologist. Participants were also prescribed to engage in exercise a minimum of $2-4$ (depending on the week of the intervention) additional times a week in the context of their own environment (e.g. in their home or through community resources) with a goal of progressing to 100 mins of moderate-intensity exercise per week midway through the intervention and $150 \mathrm{~m}$ per week by the last several weeks of the 12week intervention. Participants were instructed to self-monitor their exercise by completing a weekly exercise log

Modality of support: face-to-face and telephone

Overall contact time: 6 h 40 mins ( 4 h exercise; 2 h 40 mins smoking cessation)

Number of sessions: 20 (12 exercise, 8 smoking cessation)

Pharmacotherapy: 8 weeks nicotine patch

Quit date set?: yes

$\begin{array}{ll}\text { Outcomes } & \text { Definition of abstinence: continuous } \\ \text { Longest follow-up: } 12 \text { months } \\ \text { Biochemical validation: expired CO (using } 10 \text { ppm cut-off) }\end{array}$

Funding source National Institute on Drug Abuse (K23 DA019950) awarded to Abrantes. Pre-doctoral National Research Service Award (F31-DA035564-03) awarded to Farris

Author conflicts of interest "The authors have no conflicts of interest to report."

$\begin{array}{ll}\text { Notes } & \begin{array}{l}\text { Relevant comparisons: } 1 \text { ) reduction method versus reduction method (complementary generic health } \\ \text { education vs exercise) }\end{array}\end{array}$

\section{Risk of bias}

\begin{tabular}{lll}
\hline Bias & Authors' judgement & Support for judgement \\
\hline $\begin{array}{l}\text { Random sequence genera- } \\
\text { tion (selection bias) }\end{array}$ & Unclear risk & Method of sequence generation not reported. Urn randomisation used \\
\hline $\begin{array}{l}\text { Allocation concealment } \\
\text { (selection bias) }\end{array}$ & Unclear risk & Not reported \\
\hline
\end{tabular}


Blevins 2016 (Continued)

Blinding of outcome as- Low risk Smoking status was either biochemically verified or verified through report sessment (detection bias) from a significant other. Only 1 participant in each arm had their abstinence All outcomes verified by a significant other

Incomplete outcome data Low risk Loss to follow-up $<50 \%$ and similar between groups (attrition bias)

All outcomes

\section{Bolliger 2000a}

\begin{tabular}{|c|c|}
\hline \multirow[t]{4}{*}{ Methods } & Study design: RCT \\
\hline & Location: Switzerland \\
\hline & Setting: hospital pulmonary clinics \\
\hline & Recruitment: newspaper advertisements \\
\hline \multirow[t]{4}{*}{ Participants } & $N=400$ \\
\hline & Specialist population?: no \\
\hline & $\begin{array}{l}\text { Participant characteristics: 210/400 (53\%) female; average age: } 46.1 \text { y; average cig/day: } 29 \text {; nicotine de- } \\
\text { pendence: FTND } 5.6\end{array}$ \\
\hline & Preference for quitting abruptly versus gradually: not reported \\
\hline \multirow[t]{13}{*}{ Interventions } & $\begin{array}{l}\text { All participants were told about the general implications of smoking and its health effects. Participants } \\
\text { were asked to reduce the number of cigarettes smoked daily as much as possible; an initial reduction } \\
\text { of } 50 \% \text { was suggested. Counselling on smoking reduction was provided at each visit and smoking ces- } \\
\text { sation was recommended as the ultimate goal throughout the study }\end{array}$ \\
\hline & Comparator: placebo nicotine inhaler + reduction counselling (as above) \\
\hline & Modality of support: face-to-face \\
\hline & Overall contact time: not reported \\
\hline & Number of sessions: 12 \\
\hline & Pharmacotherapy: placebo nicotine inhaler \\
\hline & Quit date set?: no \\
\hline & Intervention: active nicotine inhaler + reduction counselling (as above) \\
\hline & Modality of support: face-to-face \\
\hline & Overall contact time: not reported \\
\hline & Number of sessions: 12 \\
\hline & Pharmacotherapy: nicotine inhaler \\
\hline & Quit date set?: no \\
\hline
\end{tabular}

Outcomes

Definition of abstinence: prolonged from week 6

Longest follow-up: $24 \mathrm{~m}$

Biochemical validation: exhaled CO (with a cut-off of $10 \mathrm{ppm}$ ) 
Bolliger 2000a (Continued)

Funding source Pharmacia and Upjohn Consumer Healthcare, Sweden

Author conflicts of interest $\quad$ "TD, ÅW, and US are all employed by Pharmacia and Upjohn, Sweden, and AR, CTB, and JPZ have received funds for research from them"

Notes Relevant comparisons: 1 ) reduction method versus reduction method (complementary pharmacotherapy versus placebo)

\section{Risk of bias}

\begin{tabular}{|c|c|c|}
\hline Bias & Authors' judgement & Support for judgement \\
\hline $\begin{array}{l}\text { Random sequence genera- } \\
\text { tion (selection bias) }\end{array}$ & Low risk & Quote: "computer generated randomisation list" \\
\hline \multirow[t]{2}{*}{$\begin{array}{l}\text { Allocation concealment } \\
\text { (selection bias) }\end{array}$} & Low risk & $\begin{array}{l}\text { Quote: "Independent pharmacists dispensed either active or placebo in- } \\
\text { halers". }\end{array}$ \\
\hline & & $\begin{array}{l}\text { Comment: Placebo inhalers were identical in appearance to intervention in- } \\
\text { halers }\end{array}$ \\
\hline \multirow[t]{2}{*}{$\begin{array}{l}\text { Blinding of participants } \\
\text { and personnel (perfor- } \\
\text { mance bias) } \\
\text { All outcomes }\end{array}$} & Unclear risk & $\begin{array}{l}\text { Quote: "Double blind, placebo controlled, randomised clinical trial"; "Indepen- } \\
\text { dent pharmacists dispensed either active or placebo inhalers according to a } \\
\text { computer generated randomisation list"; "The placebo inhalers were identical } \\
\text { in appearance and contained only menthol". }\end{array}$ \\
\hline & & $\begin{array}{l}\text { Comment: Although states that it was double-blind does not clearly state who } \\
\text { was blinded }\end{array}$ \\
\hline
\end{tabular}

Blinding of outcome as- Low risk Abstinence was biochemically validated

sessment (detection bias)

All outcomes

Incomplete outcome data Low risk

(attrition bias)

At 24-m follow-up $17 \%$ (34/200) were lost to follow-up in the intervention

All outcomes group and $28 \%(56 / 200)$ in the placebo group

Brockway 1977

\begin{tabular}{ll} 
Methods & Study design: RCT \\
& Location: USA \\
& Setting: health centre \\
& Recruitment: community newspapers and posters advertising a smoking clinic \\
\hline Participants & Specialist population?: no \\
& Participant characteristics: $13 / 27$ (48.1\%) female; age range: 18 - 50 y; average cig/day: $22 ;$ nicotine de- \\
pendence: not reported & Preference for quitting abruptly versus gradually: not reported
\end{tabular}

Interventions

Comparator: no detail given (appears to be no treatment, with participants only contacted for follow-up) 
Brockway 1977 (Continued)

Modality of support: $n / a$

Overall contact time: none

Number of sessions: none

Pharmacotherapy: none

Quit date set?: no

Intervention: Group sessions which included gradual smoking reduction by individualised situational hierarchies. Each participant formulated an 8-item situation hierarchy based on baseline smoking data collected over a week. Situations which elicited the least desire to smoke were ranked first and the most difficult ranked eighth. Over a 4-week period participants eliminated smoking by 2 hierarchical items per week, proceeding from the least to the most difficult. Sessions also comprised alternate response training, behaviour rehearsal of verbal no-smoking requests, contingency contracting (the gradual return of a USD 10 deposit based on attendance and completion of assignments), in vivo practice of non-smoking in high anxiety situations, and supplementary printed information supplied by the American Lung Association

Modality of support: face-to-face

Overall contact time: $10 \mathrm{~h} 40$ mins

Number of sessions: 8

Pharmacotherapy: none

Quit date set?: yes

\section{Outcomes}

Definition of abstinence: point prevalence

Longest follow-up: $12 \mathrm{~m}$

Biochemical validation: saliva thiocyanate

\begin{tabular}{ll}
\hline Funding source & Not reported \\
\hline Author conflicts of interest & Not reported \\
\hline Notes & Relevant comparisons: 1 ) reduction versus no treatment \\
\hline
\end{tabular}

\section{Risk of bias}

\begin{tabular}{lll}
\hline Bias & Authors' judgement & Support for judgement \\
\hline $\begin{array}{l}\text { Random sequence genera- } \\
\text { tion (selection bias) }\end{array}$ & Unclear risk & $\begin{array}{l}\text { Quote: "...smokers were randomly assigned to..." } \\
\text { Comment: no further information provided }\end{array}$ \\
\hline $\begin{array}{l}\text { Allocation concealment } \\
\text { (selection bias) }\end{array}$ & Unclear risk & No information provided \\
\hline $\begin{array}{l}\text { Blinding of outcome as- } \\
\text { sessment (detection bias) }\end{array}$ & Low risk & Smoking cessation was validated \\
$\begin{array}{l}\text { All outcomes } \\
\begin{array}{l}\text { Incomplete outcome data } \\
\text { (attrition bias) } \\
\text { All outcomes }\end{array}\end{array}$ & High risk & $\begin{array}{l}7 / 15 \text { (47\%) of the intervention group and } 7 / 12 \text { (58\%) of the comparator group } \\
\text { were lost to follow-up. Rates of dropout were therefore high overall }\end{array}$ \\
\hline
\end{tabular}




\section{Caldwell 2016}

\begin{tabular}{|c|c|}
\hline \multirow[t]{4}{*}{ Methods } & Study design: RCT \\
\hline & Location: New Zealand \\
\hline & Setting: University \\
\hline & $\begin{array}{l}\text { Recruitment: advertising through radio, newspaper, television, study website, primary care practice, } \\
\text { and smoking cessation services }\end{array}$ \\
\hline \multirow[t]{4}{*}{ Participants } & $N=502$ \\
\hline & Specialist population?: no \\
\hline & $\begin{array}{l}\text { Participant characteristics: 254/502 (51\%) female; average age: } 45.1 \text { y; average cig/day: 19; nicotine de- } \\
\text { pendence: FTND } 6.2\end{array}$ \\
\hline & Preference for quitting abruptly versus gradually: not reported \\
\hline \multirow[t]{13}{*}{ Interventions } & $\begin{array}{l}\text { All participants were advised to reduce their smoking over } 4 \text { weeks before quitting completely, and } \\
\text { used nicotine patches for } 5 \text { months after quit day. Participants were set a target quit date of } 4 \text { weeks af- } \\
\text { ter baseline, but could quit earlier if they desired }\end{array}$ \\
\hline & Comparator: smoking reduction advice + placebo nicotine inhaler. \\
\hline & Modality of support: face-to-face and telephone \\
\hline & Overall contact time: unclear \\
\hline & Number of sessions: 6 \\
\hline & $\begin{array}{l}\text { Pharmacotherapy: Placebo inhaler to use during } 4 \text {-week reduction period and the subsequent } 5 \\
\text { months ( } 6 \text { months total) and instructed "to use the inhaler when they had an urge to smoke, and to } \\
\text { have as many puffs as required to satisfy their urge (maximum of } 10 \text { puffs)." Active nicotine patches al- } \\
\text { so used for } 5 \text { months after quit day }\end{array}$ \\
\hline & Quit date set?: yes \\
\hline & Intervention: smoking reduction advice + active nicotine inhaler \\
\hline & Modality of support: face-to-face and telephone \\
\hline & Overall contact time: unclear \\
\hline & Number of sessions: 6 \\
\hline & $\begin{array}{l}\text { Pharmacotherapy: nicotine inhaler to use during } 4 \text {-week reduction period and the subsequent } 5 \\
\text { months ( } 6 \text { months total) and instructed "to use the inhaler when they had an urge to smoke, and to } \\
\text { have as many puffs as required to satisfy their urge (maximum of } 10 \text { puffs)." Nicotine patches also used } \\
\text { for } 5 \text { months after quit day }\end{array}$ \\
\hline & Quit date set?: yes \\
\hline \multirow[t]{3}{*}{ Outcomes } & Definition of abstinence: continuous \\
\hline & Longest follow-up: $6 \mathrm{~m}$ \\
\hline & Biochemical validation: exhaled $\mathrm{CO}<10 \mathrm{ppm}$ \\
\hline Funding source & The Health Research Council of New Zealand (grant number 09/199) \\
\hline Author conflicts of interest & None \\
\hline
\end{tabular}


Caldwell 2016 (Continued)

Notes

Relevant comparisons: 1 ) reduction method versus reduction method (complementary pharmacotherapy versus placebo)

\section{Risk of bias}

\begin{tabular}{lll}
\hline Bias & Authors' judgement & Support for judgement \\
\hline $\begin{array}{l}\text { Random sequence genera- } \\
\text { tion (selection bias) }\end{array}$ & Low risk & Quote: "a sequential randomization list" was used \\
\hline $\begin{array}{l}\text { Allocation concealment } \\
\text { (selection bias) }\end{array}$ & Low risk & $\begin{array}{l}\text { Quote: "a sequential randomization list that was not visible to research staff } \\
\text { or subjects. The database provided staff with a product code, which identified } \\
\text { which inhaler to give to each subject. The product codes and inhalers for both } \\
\text { treatment groups had the same appearance, both active and placebo inhalers } \\
\text { were flavored with menthol, and both subjects and staff were masked to treat- } \\
\text { ment assignment." }\end{array}$
\end{tabular}

$\begin{array}{ll}\begin{array}{l}\text { Blinding of participants } \\ \text { and personnel (perfor- }\end{array} & \text { Low risk } \\ \begin{array}{l}\text { mance bias) } \\ \text { All outcomes }\end{array} & \begin{array}{l}\text { Qutio by the trial database according to a sequential randomization list that } \\ \text { was not visible to research staff or subjects. The database provided staff with a } \\ \text { product code, which identified which inhaler to give to each subject. The prod- } \\ \text { uct codes and inhalers for both treatment groups had the same appearance, } \\ \text { both active and placebo inhalers were flavored with menthol, and both sub- } \\ \text { jects and staff were masked to treatment assignment" }\end{array}\end{array}$

\begin{tabular}{lll}
\hline $\begin{array}{l}\text { Blinding of outcome as- } \\
\text { sessment (detection bias) } \\
\text { All outcomes }\end{array}$ & Low risk & Abstinence was biochemically verified \\
\hline $\begin{array}{l}\text { Incomplete outcome data } \\
\text { (attrition bias) }\end{array}$ & Low risk & $\begin{array}{l}88 / 246(36 \%) \text { in the intervention group and } 102 / 256(40 \%) \text { in the comparator } \\
\text { group were lost to follow-up. Loss to follow-up was less than } 50 \% \text { overall and } \\
\text { similar between groups }\end{array}$ \\
\hline
\end{tabular}

\section{Carpenter 2003}

\begin{tabular}{ll}
\hline Methods & Study design: RCT \\
& Location: USA \\
& Setting: University \\
& Recruitment: print and radio advertising. All advertisements made reference to eventual quitting \\
\hline Participants & Specialist population?: no \\
& Participant characteristics: $35 / 67$ (46\%) female; average age: 43 y; average cig/day: 24; nicotine depen- \\
& dence: FTND 6 \\
& Preference for quitting abruptly versus gradually: not reported
\end{tabular}

Interventions

Comparator: Brief advice to quit was provided at the initial visit. This was modelled on 3 of the "four Rs" of the 1996 Agency for Health Care Policy and Research guidelines. If participants set a quit date they were provided with a stop-smoking booklet

Modality of support: face-to-face 
Number of sessions: 1 counselling session

Pharmacotherapy: NRT was provided to those who set a quit date. Participants returned to the lab weekly to biochemically verify abstinence and obtain further medication

Quit date set?: yes

Intervention: Following a week of self-monitoring smoking patterns, participants began a reduction programme at the second session (week 0 ). The goal was $50 \%$ reduction (or more) in cigarettes/day over 4 weeks. At each of the following sessions progress was reviewed, there was discussion of anticipated problems or obstacles, and goals were set for subsequent weeks. Once participants reached the $50 \%$ goal, they were advised to maintain this level, at a minimum. To achieve reduction participants were allowed to choose between 2 strategies (hierarchical or scheduled reduction). Hierarchical reduction involved eliminating easier cigarettes first. During the first week of reduction, participants eliminated the $25 \%$ of cigarettes they thought easiest to do without, and then gradually increased reduction to $50 \%$ after 4 weeks. Scheduled reduction involved gradually increasing the $\mathrm{ICl}$. Based on baseline smoking patterns, the counsellor calculated the average $\mathrm{ICl}$ by dividing the number of minutes the participant was awake per day by the number of cigarettes smoked. The counsellor then recalculated a minimum ICl, such that the number of cpd decreased by $25 \%$ and then by $50 \%$. Brief advice to quit was given, depending on the participant's ability to reduce during the reduction period. This was modelled on 3 of the "four Rs" of the 1996 Agency for Health Care Policy and Research guidelines. If participants set a quit date they were provided with a stop-smoking booklet.

Modality of support: face-to-face

Overall contact time: 1 h 40 mins

Number of sessions: 5 counselling sessions

Pharmacotherapy: "Participants who agreed to reduce chose between nicotine gum, patch and inhaler, or no medication. Switching type during the study was permitted, but combining NRT medications was not. Use began after the week 0 visit and could continue for the 6 months of the study".

Quit date set?: yes

$\begin{array}{ll}\text { Outcomes } & \text { Definition of abstinence: 7-day point prevalence } \\ & \text { Longest follow-up: } 6 \mathrm{~m} \\ & \text { Biochemical validation: exhaled CO (cut-off of } 10 \mathrm{ppm} \text { ) }\end{array}$

Author conflicts of interest Not reported

Notes Relevant comparisons: 1) Reduction versus abrupt

\section{Risk of bias}

\begin{tabular}{lll}
\hline Bias & Authors' judgement & Support for judgement \\
\hline $\begin{array}{l}\text { Random sequence genera- } \\
\text { tion (selection bias) }\end{array}$ & Unclear risk & No information provided \\
\hline $\begin{array}{l}\text { Allocation concealment } \\
\text { (selection bias) }\end{array}$ & Unclear risk & No information provided \\
\hline
\end{tabular}

Blinding of outcome as- Low risk Abstinence biochemically validated


Carpenter 2003 (Continued)

All outcomes

Incomplete outcome data Unclear risk Loss to follow-up not reported at 6 months
(attrition bias)
(attrition bias)

All outcomes

\section{Carpenter 2004}

Study design: RCT
Location: USA
Setting: unknown
Recruitment: proactive telephone calls through a national marketing company. The company used a
database incorporating known smokers

Participants
$\begin{aligned} & \text { S }=616 \\ & \text { Specialist population?: no } \\ & \text { Participant characteristics: } 431 / 616(85 \%) \text { female; average age: } 39 \text { y; average cig/day: } 22 ; \text { nicotine de- } \\ & \text { pendence: FTND } 5.5 \\ & \text { Preference for quitting abruptly versus gradually: not reported }\end{aligned}$

Overall contact time: 7 mins

Number of sessions: 3

Pharmacotherapy: none

Quit date set?: no

Comparator 2: Participants received counselling based on the 5 Rs of quitting: 1) relevance of smoking to the individual; 2) risks of continued smoking; 3) rewards of quitting; 4) roadblocks to success; 5) on a repeated basis, and were given brief advice to quit

Modality of support: telephone

Overall contact time: 45 mins

Number of sessions: 3

Pharmacotherapy: either nicotine gum or patch were offered alongside the advice to quit (week 6 onward). Those who set a quit date could continue using NRT

Quit date set?: yes

Intervention: Participants received instruction on 2 behavioural reduction strategies: 1) hierarchical (selectively eliminating cigarettes throughout the day) or 2) scheduled reduction (increasing the time intervals between cigarettes). They could choose how much or little to reduce and a reduction goal was set for those who wanted to reduce. At week 6 brief advice to quit was provided

Modality of support: telephone

Overall contact time: 45 mins 
Number of sessions: 3

Pharmacotherapy: either nicotine gum or patch were offered alongside reduction (weeks 0 - 6). Participants could continue use from the brief advice to quit (week 6 ) if they set a quit date

Quit date set?: yes

\begin{tabular}{|c|c|c|}
\hline Outcomes & \multirow{3}{*}{\multicolumn{2}{|c|}{$\begin{array}{l}\text { Definition of abstinence: } 7 \text {-day point prevalence } \\
\text { Longest follow-up: } 6 \mathrm{~m} \\
\text { Biochemical validation: none }\end{array}$}} \\
\hline & & \\
\hline & & \\
\hline Funding source & \multicolumn{2}{|c|}{$\begin{array}{l}\text { National Institute on Drug Abuse grant (DA 11557); National Institute on Drug Abuse grant (DA 07242) } \\
\text { to Matthew J. Carpenter; National Institute on Drug Abuse Senior Scientist Award (DA 00450) to John R. } \\
\text { Hughes }\end{array}$} \\
\hline Author conflicts of interest & \multicolumn{2}{|l|}{ Not reported } \\
\hline Notes & \multicolumn{2}{|c|}{ Relevant comparisons: 1) Reduction versus no treatment; 2) Reduction versus abrupt } \\
\hline \multicolumn{3}{|l|}{ Risk of bias } \\
\hline Bias & Authors' judgement & Support for judgement \\
\hline $\begin{array}{l}\text { Random sequence genera- } \\
\text { tion (selection bias) }\end{array}$ & Unclear risk & No information given \\
\hline $\begin{array}{l}\text { Allocation concealment } \\
\text { (selection bias) }\end{array}$ & Unclear risk & No information given \\
\hline $\begin{array}{l}\text { Blinding of outcome as- } \\
\text { sessment (detection bias) } \\
\text { All outcomes }\end{array}$ & High risk & No biochemical verification and the amount of contact between arms differed \\
\hline $\begin{array}{l}\text { Incomplete outcome data } \\
\text { (attrition bias) } \\
\text { All outcomes }\end{array}$ & Unclear risk & Not reported \\
\hline
\end{tabular}

\section{Chan 2011}

\begin{tabular}{|c|c|}
\hline \multirow[t]{4}{*}{ Methods } & Study design: RCT \\
\hline & Location: China \\
\hline & Setting: unclear \\
\hline & $\begin{array}{l}\text { Recruitment: local media advertisements and contact with previous cohorts of smokers who had had } \\
\text { cessation counselling but failed to quit }\end{array}$ \\
\hline \multirow[t]{4}{*}{ Participants } & $N=1154$ \\
\hline & Specialist population?: no \\
\hline & $\begin{array}{l}\text { Participant characteristics: 208/1154 (18\%) female; average age: } 42 \text { y; average cig/day: 20; nicotine de- } \\
\text { pendence: FTND } 5.2\end{array}$ \\
\hline & Preference for quitting abruptly versus gradually: not reported \\
\hline
\end{tabular}


Chan 2011 (Continued) Interventions
Comparator 1: Simple cessation advice: self-help quitting pamphlet, plus 10 mins of simple advice on the health hazards of smoking and the importance of smoking cessation

Modality of support: face-to-face

Overall contact time: 10 mins

Number of sessions: 1

Pharmacotherapy: none

Quit date set?: no

Intervention 1: Smoking reduction and cessation counselling: individual counselling in smoking reduction, plus a self-help quitting pamphlet. "Specific SR counselling emphasized achieving the ultimate goal of complete cessation by focusing on the importance of SR before quitting, how reduction is useful and effective when quitting is difficult, and on how to reduce".

Modality of support: face-to-face

Overall contact time: 30 mins

Number of sessions: 3

Pharmacotherapy: 8-week supply of either nicotine gum or patches starting at baseline

Quit date set?: no

Intervention 2: Smoking reduction and cessation counselling + NRT adherence counselling: individual counselling in smoking reduction and adherence to NRT, plus a self-help quitting pamphlet. "Specific SR counselling emphasized achieving the ultimate goal of complete cessation by focusing on the importance of SR before quitting, how reduction is useful and effective when quitting is difficult, and on how to reduce".

Modality of support: face-to-face

Overall contact time: 30 mins

Number of sessions: 3

Pharmacotherapy: 8-week supply of either nicotine gum or patches starting at baseline

Quit date set?: no

\section{Outcomes}

Definition of abstinence: 7-day point prevalence

Longest follow-up: $6 \mathrm{~m}$

Biochemical validation: urinary cotinine $(<115 \mathrm{ng} / \mathrm{ml})$ and exhaled CO (<9 ppm)

Funding source

Health and Health Services Research Fund, Hong Kong SAR (Project no. 01030611). Nicotine patches/gum provided free of charge by Pfizer (later named McNeil AB)

Author conflicts of interest "None of the authors have any connections to the tobacco, alcohol, pharmaceutical, gaming industries, or anyone substantially funded by one of these organizations"

Notes

Relevant comparisons: 1) Reduction versus abrupt; 2) Reduction method versus reduction method (complementary NRT adherence counselling)

\section{Risk of bias}


Chan 2011 (Continued)

Random sequence genera- Low risk tion (selection bias)
Quote: "The random numbers for group assignment were generated by the research assistant (not the counsellors) of the project using a personal computer before subject recruitment"

\section{Allocation concealment Low risk} (selection bias)
Quote: "Randomization was performed by opening of a serially labelled, opaque and sealed envelope with a card inside indicating the randomly allocated group by a trained smoking cessation counsellor"

Blinding of outcome as- Low risk Abstinence was biochemically validated

sessment (detection bias)

All outcomes

Incomplete outcome data Low risk

(attrition bias)

$52 / 479(11 \%)$ in reduction + adherence group, $44 / 449(10 \%)$ in reduction

All outcomes group, and $10 / 226(4 \%)$ in simple cessation group were lost to follow-up. Attrition rates were low and similar across groups

\section{Cinciripini 1995}

$\begin{array}{ll}\text { Methods } & \text { Study design: RCT } \\ \text { Location: USA } & \text { Setting: unclear } \\ \text { Recruitment: from the community; no further detail given }\end{array}$

Participants $\quad \mathrm{N}=128$

Specialist population?: no

Participant characteristics: 74/128 (58\%) female; average age: 45 y; average cig/day: 24; nicotine dependence: FTND 6.1

Preference for quitting abruptly versus gradually: not reported

All participants received a cognitive behavioural counselling intervention in weeks 2 - 5 on adherence, physiological and psychological effects of nicotine, deep-breathing exercises, and acquiring behaviours incompatible with smoking, such as reviewing reasons for quitting list, repeating certain coping phrases or learning to change environment in response to urges. Participants were supported to quit in week 5. Relapse prevention counselling took place weeks 5 - 9, emphasising maintenance for those who quit previously and cessation for those who did not

Comparator 1: Scheduled non-reduction: participants were told to smoke at specific times allowing for no adjustment to interval or cigarette consumption between weeks 2 and 5

Modality of support: face-to-face

Overall contact time: $2 \mathrm{~h}$

Number of sessions: 9

Pharmacotherapy: none

Quit date set?: yes

Comparator 2: Non-scheduled non-reduction: participants continued to smoke as usual before attempting to quit at week 5

Modality of support: face-to-face 
Cinciripini 1995 (Continued)

Overall contact time: $2 \mathrm{~h}$

Number of sessions: 9

Pharmacotherapy: none

Quit date set?: yes

Intervention 1: Scheduled reduction: instructed to smoke only at specific times between weeks 2 and 5 , with the $\mathrm{ICl}$ progressively lengthened. Smoking was to take place only in the first 5 minutes of the ICl; any missed cigarettes could not be accumulated for later use. In weeks 2 and $3 \mathrm{ICls}$ were set by dividing $2 / 3$ and $1 / 3$ (respectively) of a participant's average baseline of $24 \mathrm{cpd}$ over $16 \mathrm{~h}$. In week 4 consumption reduced by $1 / 3$ of the rate for week 2 every day, until consumption reached 2 - $4 \mathrm{cpd}$. Duration of the reduction period was 3 weeks

Modality of support: face-to-face

Overall contact time: $2 \mathrm{~h}$

Number of sessions: 9

Pharmacotherapy: none

Quit date set?: yes

Intervention 2: Non-scheduled reduction: participants gradually reduced cpd using the same quota as for scheduled reduced, but ICls were not provided and participants could choose when they smoked the cigarettes. Duration of the reduction period was 3 weeks

Modality of support: face-to-face

Overall contact time: $2 \mathrm{~h}$

Number of sessions: 9

Pharmacotherapy: none

Quit date set?: yes

Outcomes

Definition of abstinence: prolonged abstinence (allowing up to 5 lapses between study assessments)

Longest follow-up: $12 \mathrm{~m}$

Biochemical validation: cotinine $(<14 \mathrm{ng} / \mathrm{ml})$

The National Institute of Drug Abuse: grants DHHS DA-04520 and DHHS DA-02507

Author conflicts of interest Not reported

Notes Relevant comparisons: 1) Reduction versus abrupt; 2) Reduction method versus reduction method (scheduled versus non-scheduled)

\section{Risk of bias}

\begin{tabular}{lll}
\hline Bias & Authors' judgement & Support for judgement \\
\hline $\begin{array}{l}\text { Random sequence genera- } \\
\text { tion (selection bias) }\end{array}$ & Unclear risk & No information provided \\
\hline $\begin{array}{l}\text { Allocation concealment } \\
\text { (selection bias) }\end{array}$ & Unclear risk & No information provided \\
\hline
\end{tabular}


Cinciripini 1995 (Continued)

Blinding of outcome as- Low risk Abstinence was biochemically verified sessment (detection bias)

All outcomes

Incomplete outcome data Unclear risk Not reported
(attrition bias)

(attrition bias)

All outcomes

\section{Cinciripini 2006}

\begin{tabular}{ll} 
Methods & Study design: RCT \\
& Location: USA \\
& Setting: not reported \\
& Recruitment: from the community, no further detail given \\
\hline Participants & N = "Over $700 "$ \\
& Specialist population?: not reported \\
& Participant characteristics: not reported \\
& Preference for quitting abruptly versus gradually: not reported
\end{tabular}

Interventions

Comparator 1: Usual care control: participants were instructed to quit smoking within a few days of study entry and begin using the nicotine patch on their quit day. They were provided with no instructions to reduce

Modality of support: unclear

Overall contact time: unclear

Number of sessions: unclear

Pharmacotherapy: nicotine patches from quit day

Quit date set?: unclear

Intervention 1: Scheduled smoking: participants' smoking was scheduled using a handheld computer, which signalled smoking at progressively increasing $\mathrm{ICl}$. Participants were instructed to begin using nicotine patches on their quit day

Modality of support: unclear

Overall contact time: unclear

Number of sessions: unclear

Pharmacotherapy: nicotine patches from quit day

Quit date set?: unclear

Intervention 2: Scheduled smoking + concurrent nicotine patches: participants' smoking was scheduled using a handheld computer, which signalled smoking at progressively increasing ICls. Participants were instructed to use nicotine patches during the reduction period, continuing after their quit day

Modality of support: unclear

Overall contact time: unclear 
Cinciripini 2006 (Continued)

Number of sessions: unclear

Pharmacotherapy: nicotine patches during reduction and after quit day

Quit date set?: unclear

Outcomes Definition of abstinence: not reported

Longest follow-up: unclear. "long-term follow-up" was carried out, but we do not have access to the da-

ta

Biochemical validation: not reported

\begin{tabular}{|c|c|}
\hline Funding source & Not reported \\
\hline Author conflicts of interest & Not reported \\
\hline Notes & $\begin{array}{l}\text { Relevant comparisons: 1) Reduction versus abrupt 2) Reduction method versus reduction method (pre- } \\
\text { quit nicotine patch) }\end{array}$ \\
\hline & $\begin{array}{l}\text { This study has only been published as an abstract and therefore limited information is available. } \\
\text { Through previous contact with the author in } 2010 \text { we learnt that the long-term abstinence rates were } \\
\text { being analysed at that time. However, we are not aware that these have been published since, and re- } \\
\text { cent contact with the author was unsuccessful. }\end{array}$ \\
\hline
\end{tabular}

\section{Risk of bias}

\begin{tabular}{lll}
\hline Bias & Authors' judgement & Support for judgement \\
\hline $\begin{array}{l}\text { Random sequence genera- } \\
\text { tion (selection bias) }\end{array}$ & Unclear risk & Not described \\
\hline $\begin{array}{l}\text { Allocation concealment } \\
\text { (selection bias) }\end{array}$ & Unclear risk & Not described \\
\hline $\begin{array}{l}\text { Blinding of outcome as- } \\
\text { sessment (detection bias) } \\
\text { All outcomes }\end{array}$ & Unclear risk & Unclear whether abstinence was biochemically verified \\
\hline $\begin{array}{l}\text { Incomplete outcome data } \\
\text { (attrition bias) } \\
\text { All outcomes }\end{array}$ & Unclear risk & Attrition not reported \\
\hline $\begin{array}{l}\text { Other bias } \\
\end{array}$ & Unclear risk & $\begin{array}{l}\text { Long-term abstinence was measured but has not been reported. Insufficient } \\
\text { information to judge whether this is as a result of selective reporting }\end{array}$ \\
\hline
\end{tabular}

Cook 2016

Study design: factorial RCT (4 factors, 16 trial arms)
Location: USA
Setting: primary care clinics
Recruitment: participants were invited during primary care clinic visits to participate in a research pro-
gramme to help them to reduce their smoking. Those interested were referred electronically to the re-
search office


Cook 2016 (Continued)

Participants $\quad \mathrm{N}=517$

Specialist population?: no

Participant characteristics: 328/517 (63.4\%) female; average age: 47 y; average cig/day: 18; nicotine dependence: FTND 4.8

Preference for quitting abruptly versus gradually: not reported

Interventions
All participants could elect to receive cessation-phase treatment, which consisted of 8 weeks of nicotine patch and gum treatment and 2 brief phone counselling sessions at any point throughout the treatments described below

Intervention factors:

1. Motivational interviewing (MI): initial 20-min in-person counselling session followed by 3 bi-weekly, 10-min counselling calls over the 6 -week intervention period. Based on the principles developed by Miller \& Rollnick, the counselling sessions included motivation-building exercises to reinforce intrinsic motivation and to help participants overcome ambivalence about quitting. Case managers engaged participants in a series of motivation-building exercises such as reviewing feelings and thoughts about the pros and cons of quitting and smoking, reinforcing the positives of quitting, helping to dispel myths and concerns about the negatives of quitting, and posing questions about the "good" aspects of smoking.

2. Behavioural smoking reduction counselling: Initial 20-min in-person counselling session followed by 6 weekly 10-min counselling calls. During these sessions, participants set smoking reduction goals and developed reduction strategies (e.g. delaying smoking, eliminating smoking in specific situations). Participants were also instructed to record daily smoking, which case managers used to identify successes and challenges

3. Nicotine gum: participants were instructed to use $2 \mathrm{mg}$ gum for the 6 -week intervention period ( $\geq 9$ per day, 1 piece per $1-2 \mathrm{~h}$ ) in place of smoking

4. Nicotine patch: participants were instructed to use $14 \mathrm{mg}$ patches daily for the 6-week intervention period

Where all intervention factors were OFF this resulted in a 'no treatment' condition

Modality of support: face-to-face and telephone

Overall contact time: 50 mins for Ml / 1 h 20 mins for reduction

Number of sessions: 4 MI sessions / 7 reduction sessions

Pharmacotherapy: as described above. The nicotine gum and nicotine patch factor provided NRT over a 6-week period and if participants elected to enter the cessation phase at any point they were provided with 8 weeks of gum and patch treatment

Quit date set?: no, all participants could elect to enter the cessation phase whenever they wanted during the treatment

Longest follow-up: $6 \mathrm{~m}$

Biochemical validation: none

The National Cancer Institute (grants 9P50CA143188 and 1K05CA139871). Work was carried out in part while TRS was a Primary Care Research Fellow supported by a National Research Service Award (T32HP10010) from the Health Resources and Services Administration. WYL was also supported by the NSF (grant DMS-1305725). LMC was also supported by the NIH (grants P50DA10075 and R01DK097364). JWC was also supported by a Merit Review Award (101CX00056) from the US Department of Veterans Affairs. 
Cook 2016 (Continued)

Author conflicts of interest "The authors have received no direct or indirect funding from, nor do they have a connection with, the tobacco, alcohol, pharmaceutical or gaming industries or anybody funded substantially by one of these organizations. W.-Y.L. is supported partially by a grant from Eli Lilly and Company for research that is unrelated to smoking or tobacco dependence treatment."

\section{Notes}

Relevant comparisons: 1) Reduction versus no treatment; 2) Reduction versus abrupt; 3) Reduction method versus reduction method (varying by use and type of pharmacotherapy)

In line with guidance in the Cochrane Handbook we looked for potential interactions between the factors in this factorial trial. An interaction between the MI and nicotine gum factor is reported by the authors. Rather than exclude data from this trial from analyses, which we believe would introduce bias, we account for the risk of bias potentially introduced by this interaction in our 'Risk of bias' assessment below and carried out sensitivity analyses removing it from analyses alongside other studies judged to be at high risk of bias.

For the reduction versus no treatment analyses we compared all the study arms including the reduction intervention with the 1 study arm that received no smoking cessation treatment (no reduction, MI or NRT). For the analysis comparing reduction to abrupt quitting we compare any study arms receiving reduction alone or alongside any other study components (MI and/or NRT) with any study arms that did not receive the reduction component, but received another form of cessation support. This study is also included in the analysis comparing reduction treatment + pharmacotherapy with reduction treatment alone - all study arms that provide reduction advice alongside either patch, gum or a combination of the 2 are compared with all study arms that provide reduction advice without any pharmacotherapy.

Where relevant we have ensured that study arms that received $\mathrm{MI}$ and nicotine gum in combination have been entered into analyses separately, to study arms that did not receive the combination of MI and nicotine gum.

\section{Risk of bias}

\begin{tabular}{lll}
\hline Bias & Authors' judgement & Support for judgement \\
\hline $\begin{array}{ll}\text { Random sequence genera- } \\
\text { tion (selection bias) }\end{array}$ & Low risk & $\begin{array}{l}\text { Participants were randomised to treatment conditions using stratified permut- } \\
\text { ed, computer-generated block randomisation; stratified by gender and clinic } \\
\text { with a fixed block size of } 16 \text { based on the 16 unique possible combinations of } \\
\text { intervention components (in random order within each block) }\end{array}$ \\
\hline
\end{tabular}

\begin{tabular}{|c|c|c|}
\hline $\begin{array}{l}\text { Allocation concealment } \\
\text { (selection bias) }\end{array}$ & Low risk & $\begin{array}{l}\text { Participants were randomised to treatment conditions using stratified permut- } \\
\text { ed, computer-generated block randomisation; stratified by gender and clinic } \\
\text { with a fixed block size of } 16 \text { based on the } 16 \text { unique possible combinations of } \\
\text { intervention components (in random order within each block) }\end{array}$ \\
\hline
\end{tabular}

Blinding of participants High risk and personnel (performance bias)

All outcomes
Quote: "This $2 \times 2 \times 2 \times 2$ factorial experiment had four factors each comprising an active (ON) condition and control (OFF) condition: 1) Nicotine Patch; 2) Nicotine Gum; 3) Ml; and 4) BR Counseling, yielding 16 unique experimental conditions".

Comment: Placebo was not used for the OFF nicotine patch or nicotine gum factor
Self-report (no biochemical validation). The lack of MI meant that participants had less face-to-face contact and less intensive support in some of the comparison trial arms

$\begin{array}{lll}\text { Blinding of outcome as- } & \text { High risk } & \text { Self-report } \\ \text { sessment (detection bias) } & & \text { had less face-to-fa } \\ \text { All outcomes } & \text { parison trial arms }\end{array}$

Incomplete outcome data Low risk

(attrition bias)

There were no significant differences in missing data across the contrasting levels of each intervention factor. $17 \%(45 / 260)$ in the behavioural reduction counselling ON group, and 15\% (38/257) in the behavioural reduction counselling OFF group were lost to follow-up 
Cook 2016 (Continued)

Other bias High risk This is a factorial trial and we found an interaction between the MI and nicotine gum factors. This was not an a priori-hypothesised interaction, and challenges the assumption that the factors studied were independent.

$\begin{array}{ll}\text { Methods } & \text { Study design: factorial RCT }(2 \times 2) \\ & \text { Location: USA } \\ & \text { Setting: } n / a \text { (remote intervention) } \\ & \text { Recruitment: callers to hotline telephone number, advertised through direct mailings, posters and } \\ \text { brochures, radio talk shows, radio ads, newspaper articles and ads, and television announcements. Ad- } \\ \text { vert read 'If you want to quit smoking, we can help' } \\ \text { N }=1895 \\ \text { Specialist population?: no } \\ \text { Participant characteristics: } 1232 / 1895 \text { (15\%) female; average age: } 42 \text { y; average cig/day: } 28 ; \text { nicotine } \\ \text { dependence (average years smoked): } 24 \mathrm{y} \\ \text { Preference for quitting abruptly versus gradually: not reported }\end{array}$

All participants received the following interventions in the form of self-help booklets sent through the post

Comparator 1: Control booklet (not eligible as not a quitting intervention, but can not be classed as no smoking cessation treatment): provided information on the health hazards of smoking and the nature of tobacco addiction, but did not give specific advice on how to stop smoking (15 pages)

Modality of support: self-help booklet

Overall contact time: $\mathrm{n} / \mathrm{a}$

Number of sessions: $\mathrm{n} / \mathrm{a}$

Pharmacotherapy: none

Quit date set?: unclear whether booklet indicated when to quit

Comparator 2: Cold-turkey: high structure: booklet (45 - 58 pages) instructed participants to quit abruptly. Day-by-day format. Participants were instructed to read the booklet every day and to carry out the activities given for each day of the plan

Modality of support: self-help booklet

Overall contact time: $\mathrm{n} / \mathrm{a}$

Number of sessions: $\mathrm{n} / \mathrm{a}$

Pharmacotherapy: none

Quit date set?: unclear whether booklet indicated when to quit

Comparator 3: Cold-turkey: low structure: booklet (45 - 58 pages) instructed participants to quit abruptly. The same advice was provided as in the high-structure group, i.e. tips in the same sequence; however, this was not in a structured form, and participants were instructed to examine the menu of information and to select those exercises they felt would be helpful rather than working through them systematically 
Modality of support: self-help booklet

Overall contact time: $\mathrm{n} / \mathrm{a}$

Number of sessions: $\mathrm{n} / \mathrm{a}$

Pharmacotherapy: none

Quit date set?: unclear whether booklet indicated when to quit

Intervention 1: Gradual - high structure: booklet (45 - 58 pages) instructed smokers to gradually reduce the number of cigarettes smoked over a brief period before quitting altogether. Participants were given a number of suggestions of how to reduce, such as setting daily goals, switching brands, changing habits, and delaying the first cigarette of the day. Participants were instructed to read the booklet every day and to carry out the activities given for each day of the plan

Modality of support: self-help booklet

Overall contact time: $\mathrm{n} / \mathrm{a}$

Number of sessions: $\mathrm{n} / \mathrm{a}$

Pharmacotherapy: none

Quit date set?: unclear whether booklet indicated when to quit

Intervention 2: Gradual - low structure: booklets (45 - 58 pages) instructed smokers to gradually reduce the number of cigarettes smoked over a brief period before quitting altogether. Participants were given a number of suggestions on how to reduce, such as setting daily goals, switching brands, changing habits, and delaying the first cigarette of the day. The same advice was given as tips in the same sequence as in the high-structure booklet, but not in a structured form. Participants were instructed to examine the menu of information and to select those exercises they felt would be helpful rather than working through them systematically

Modality of support: self-help booklet

Overall contact time: $\mathrm{n} / \mathrm{a}$

Number of sessions: $\mathrm{n} / \mathrm{a}$

Pharmacotherapy: none

Quit date set?: unclear whether booklet indicated when to quit

Outcomes

Definition of abstinence: continuous

Longest follow-up: $6 \mathrm{~m}$

Biochemical validation: none

\section{Funding source}

The National Cancer Institute (CA36265)

Author conflicts of interest Not reported

Notes

Relevant comparisons: (excluding control booklet study arm) 1) Reduction versus abrupt; 2) Reduction method versus reduction method (structured versus unstructured)

Factorial RCT, but no interaction detected between factors

\section{Risk of bias}


Cummings 1988 (Continued)

Random sequence genera- Low risk tion (selection bias)

Quote: "randomization was done from a pre-randomized list so subjects were randomized as they called into the study and were defined as eligible" (email communication).

\begin{tabular}{|c|c|c|}
\hline $\begin{array}{l}\text { Allocation concealment } \\
\text { (selection bias) }\end{array}$ & Low risk & $\begin{array}{l}\text { Self-help intervention, involving minimal contact with investigators/enrolling } \\
\text { clinicians }\end{array}$ \\
\hline
\end{tabular}

\begin{tabular}{|c|c|c|}
\hline $\begin{array}{l}\text { Blinding of outcome as- } \\
\text { sessment (detection bias) } \\
\text { All outcomes }\end{array}$ & Low risk & $\begin{array}{l}\text { No biochemical verification; however, there was no contact with researchers } \\
\text { and the relevant study groups (excluding control booklet study arm) did not } \\
\text { differ in intensity }\end{array}$ \\
\hline
\end{tabular}

\begin{tabular}{|c|c|c|}
\hline $\begin{array}{l}\text { Incomplete outcome data } \\
\text { (attrition bias) } \\
\text { All outcomes }\end{array}$ & Unclear risk & $\begin{array}{l}19.1 \% \text { of total randomised lost to follow-up; reported not to vary by arm. } \\
\text { However, } 18 \text { additional participants are missing from the report results table. } \\
\text { These participants are included in the current analyses and treated as non-ab- } \\
\text { stainers, but their allocation to treatment arms is unknown }\end{array}$ \\
\hline
\end{tabular}

\section{Curry 1988}

\section{Methods}

Study design: factorial RCT $(2 \times 2)$

Location: USA

Setting: community

Recruitment: radio announcements and newspaper advertisement

Participants
$\begin{aligned} & \text { N }=139 \\ & \text { Specialist population?: no } \\ & \text { Participant characteristics: } 71 / 139(51 \%) \text { female; average age: } 40.6 \text { y; average cig/day: } 28 ; \text { nicotine de- } \\ & \text { pendence: not reported } \\ & \text { Preference for quitting abruptly versus gradually: not reported }\end{aligned}$

Interventions

Comparator 1: relapse prevention (abrupt); group-based: participants were advised to quit abruptly in the third group session. Participants were encouraged instead to think of quitting as a gradual skills-acquisition process in which the most difficult task is staying off cigarettes rather than initially quitting

Modality of support: face-to-face

Overall contact time: $16 \mathrm{~h}$

Number of sessions: 8

Pharmacotherapy: none

Quit date set?: yes

Comparator 2: relapse prevention (abrupt); self-help: participants were advised to quit abruptly. Participants were encouraged instead to think of quitting as a gradual skills-acquisition process in which the most difficult task is staying off cigarettes rather than initially quitting. The self-help group received 8 units of materials (work books with written exercises) in total

Modality of support: self-help booklets

Overall contact time: $\mathrm{n} / \mathrm{a}$

Number of sessions: $\mathrm{n} / \mathrm{a}$ 
Curry 1988 (Continued)

Pharmacotherapy: none

Quit date set?: yes

Intervention 1: absolute abstinence (reduction); group-based: focused on the gradual acquisition of coping skills in face-to- face group sessions. Smoking cessation was defined as a gradual process of withdrawal from nicotine. Absolute abstinence was enforced with a contingency contract, where participants sent a cheque for USD 15 to a person or organisation that they disliked if they were not successful

Modality of support: face-to-face

Overall contact time: $16 \mathrm{~h}$

Number of sessions: 8

Pharmacotherapy: none

Quit date set?: yes

Intervention 2: absolute abstinence (reduction); self-help: focused on the gradual acquisition of coping skills. Smoking cessation was defined as a gradual process of withdrawal from nicotine. Absolute abstinence was enforced with a contingency contract, where participants sent a cheque for USD 15 to a person or organisation that they disliked if they were not successful. The self-help group received $8 t$ units of materials (work books with written exercises) in total

Modality of support: self-help booklets

Overall contact time: $\mathrm{n} / \mathrm{a}$

Number of sessions: $n / a$

Pharmacotherapy: none

Quit date set?: yes

Biochemical validation: salivary thiocyanate

\section{Author conflicts of interest Not reported}

\section{Notes}

Relevant comparisons: 1) Reduction versus abrupt; 2) Reduction method versus reduction method (modality)

Factorial RCT, but no interaction detected between factors

\section{Risk of bias}

\begin{tabular}{lll}
\hline Bias & Authors' judgement & Support for judgement \\
\hline $\begin{array}{l}\text { Random sequence genera- } \\
\text { tion (selection bias) }\end{array}$ & Low risk & $\begin{array}{l}\text { Quote: "Within each stratum (availability for day or evening meetings) a total } \\
\text { of 24 participants were picked randomly and grouped into pairs of } 12 . "\end{array}$ \\
& $\begin{array}{l}\text { Quote: "Random numbers table" } \\
\text { Quote: "A coin toss determined assignment to RP or AA With persons partic- } \\
\text { ipating together one person was randomised and the other person non ran- }\end{array}$
\end{tabular}


Curry 1988 (Continued)

domly assigned to the same to the same program but not necessarily to the same format."

\begin{tabular}{|c|c|c|}
\hline $\begin{array}{l}\text { Allocation concealment } \\
\text { (selection bias) }\end{array}$ & Unclear risk & No information provided \\
\hline \multirow{3}{*}{$\begin{array}{l}\text { Incomplete outcome data } \\
\text { (attrition bias) } \\
\text { All outcomes }\end{array}$} & & $\begin{array}{l}\text { Quote: "Overall, significantly fewer participants assigned to the self-help for- } \\
\text { mat began treatment (group }=87.5 \% \text {, self-help }=59.3 \% \text { )"; }\end{array}$ \\
\hline & & $\begin{array}{l}\text { Quote: "Similarly, more participants who began treatment in the group format } \\
\text { tended to complete treatment (group }=76.2 \% \text {, self-help }=59.3 \% \text { )"; }\end{array}$ \\
\hline & & $\begin{array}{l}\text { Quote: "There were no significant differences in participation rates between } \\
\text { the RP and AA program types in either the group or self-help format" }\end{array}$ \\
\hline
\end{tabular}

\section{Dooley 1992}

$\begin{array}{ll}\text { Methods } & \text { Study design: RCT } \\ \text { Location: Australia } \\ \text { Setting: unclear } \\ \text { Recruitment: through mass media announcements }\end{array}$

Participants
$\begin{aligned} & \text { S }=92 \\ & \text { Specialist population?: no } \\ & \text { Participant characteristics: } 43 / 92(47 \%) \text { female; average age: } 38.5 \text { y; average cig/day: 26; nicotine de- } \\ & \text { pendence: FTND } 7.3 \\ & \text { Preference for quitting abruptly versus gradually: not reported }\end{aligned}$

Comparator 1: Nicotine fading + relapse prevention training: participants were advised to switch brands at the end of weeks 1,2 , and 3 to brands with lower nicotine content to effect reductions of $30 \%, 60 \%$ and $90 \%$ from baseline respectively. Participants were instructed to smoke as they normally would. Participants also received counselling focused on preparing to stop smoking and then maintaining abstinence

Modality of support: face-to-face

Overall contact time: $9 \mathrm{~h}$

Number of sessions: 6

Pharmacotherapy: none

Quit date set?: yes

Comparator 2: Minimal contact control (not eligible for inclusion): participants were given a package of publicly-available written materials on smoking hazards and ways to quit. Baseline cigarette tally was reported back to investigators by mail. No contact was made with participants during the time the oth- 
Dooley 1992 (Continued)

er groups were receiving treatment. After the 3-month follow-up participants were offered a choice of either of the active treatment strategies

Modality of support: written materials

Overall contact time: $\mathrm{n} / \mathrm{a}$

Number of sessions: $\mathrm{n} / \mathrm{a}$

Pharmacotherapy: none

Quit date set?: unclear

Intervention: Nicotine gum + relapse prevention training: participants used nicotine gum ( $2 \mathrm{mg})$, supplied free of charge, as needed. For the first 3 weeks, participants were instructed to use the gum whenever they felt the urge to smoke and encouraged to reduce the number of cigarettes smoked. At the end of the last treatment session, they were advised to slowly fade out gum use. Free gum was supplied up to 3 months post-treatment upon request. Participants also received counselling focused on preparing to stop smoking and then maintaining abstinence

Modality of support: face-to-face

Overall contact time: $9 \mathrm{~h}$

Number of sessions: 6

Pharmacotherapy: nicotine gum (2 mg) for approximately 4 months

Quit date set?: yes

Outcomes

Definition of abstinence: 7-day point prevalence

Longest follow-up: $12 \mathrm{~m}$ (minimal contact group only followed up to $3 \mathrm{~m}$ )

Biochemical validation: saliva thiocyanate (concentration of $\leq 85 \mathrm{pg} / \mathrm{d}$ were classified as abstinent)

Funding source

The Behaviour Research and Therapy Centre, University of Queensland (Grant 85002). "Nicotine gum was supplied by the Glaxo Corporation"

\section{Author conflicts of interest Not reported}

Notes Relevant comparisons: compares behavioural reduction to nicotine fading. This is not included in the MA of reduction versus abrupt but is summarised separately.

The minimal contact group was not followed up to $12 \mathrm{~m}$ like the other groups so this is not eligible for the review or data analysis

\section{Risk of bias}

Bias Authors' judgement Support for judgement

Random sequence genera- Unclear risk tion (selection bias)

Quote: "Subjects were assigned to one of three treatment groups using a weighted random assignment procedure".

Comment: No further information reported

\begin{tabular}{|c|c|c|}
\hline $\begin{array}{l}\text { Allocation concealment } \\
\text { (selection bias) }\end{array}$ & Unclear risk & $\begin{array}{l}\text { Quote: "Subjects were assigned to one of three treatment groups using a } \\
\text { weighted random assignment procedure". }\end{array}$ \\
\hline
\end{tabular}

Comment: No further information reported.

Blinding of outcome as-
sessment (detection bias) $\quad$ Low risk $\quad$ Abstinence was biochemically verified


Dooley 1992 (Continued)

All outcomes

Incomplete outcome data High risk (attrition bias)

All outcomes
$26 / 38(32 \%)$ of the nicotine gum and $15 / 17(12 \%)$ of the minimal contact group did not complete treatment, meaning there was differential dropout between groups

\section{Ebbert 2015}

Study design: RCT
Location: Australia, Canada, Czech Republic, Egypt, Germany, Japan, Mexico, Taiwan, UK, USA
Setting: clinical trial centres, academic centres and outpatient clinics
Recruitment: "through advertising"

Participants N=1510

Specialist population?: no

Participant characteristics: 659/1510 (44\%) female; average age: 44.6 y; average cig/day: 21; nicotine dependence: FTND 5.6

Preference for quitting abruptly versus gradually: not reported

Interventions

All participants received a self-help smoking cessation booklet and were asked to reduce baseline smoking rate by $\geq 50 \%$ by week 4 with further reduction to $75 \%$ from baseline by week 8 with the goal of quitting by week 12. Participants could reduce their smoking faster and could make a quit attempt prior to week 12 if desired. Advice on reduction techniques was provided, such as systematically increasing the amount of time between cigarettes and rank-ordering cigarettes from easiest to hardest to give up, and giving up the easiest to the hardest. Participants who had not reduced or made a quit attempt by week 12 were encouraged to continue medications and visits and make quit attempts, and participants who relapsed after week 12 were encouraged to make new quit attempts

Comparator: Placebo

Modality of support: face-to-face and telephone

Overall contact time: up to $4 \mathrm{~h} 40$ mins

Number of sessions: 28 (18 face-to-face, 10 phone calls)

Pharmacotherapy: placebo varenicline ( 24 weeks: $0.5 \mathrm{mg}$ once daily for 3 days, increasing to $0.5 \mathrm{mg}$ twice daily for days 4 to 7 , and then to the maintenance dose of $1 \mathrm{mg}$ twice daily)

Quit date set?: yes

Intervention: Varenicline

Modality of support: face-to-face and telephone

Overall contact time:up to $4 \mathrm{~h} 40$ mins

Number of sessions: 28 (18 face-to-face, 10 phone calls)

Pharmacotherapy: varenicline ( 24 weeks: $0.5 \mathrm{mg}$ once daily for 3 days, increasing to $0.5 \mathrm{mg}$ twice daily for days 4 to 7 , and then to the maintenance dose of $1 \mathrm{mg}$ twice daily)

Quit date set?: yes 
Ebbert 2015 (Continued)

$$
\begin{aligned}
& \text { Longest follow-up: } 1 \text { year } \\
& \text { Biochemical validation: exhaled } \mathrm{CO}(\leq 10 \mathrm{ppm} \text { at each visit) }
\end{aligned}
$$

\begin{tabular}{|c|c|}
\hline Funding source & Pfizer Inc \\
\hline Author conflicts of interest & $\begin{array}{l}\text { "Dr. Ebbert reports grants from Pfizer, Orexigen and JHP Pharmaceuticals and personal fees from } \\
\text { GlaxoSmithKline during the conduct of the study. Dr. Hughes reports personal fees from Alere/Free } \\
\text { and Clear, Equinox, GlaxoSmithKline, Healthwise, Pfizer, Embera, Selecta, DLA Piper, Dorrffermey- } \\
\text { er, Nicoventures, Pro Ed, Publicis, Cicatelli, and non-financial support from Swedish Match, outside } \\
\text { the submitted work. Dr. West reports grants, personal fees and non-financial support from Pfizer, } \\
\text { GlaxoSmithKline, and Johnson \& Johnson outside the submitted work. Dr. Rennard reports person- } \\
\text { al fees from Almirall, Novartis, Nycomed, Pfizer, A2B Bio, Dalichi Sankyo, APT Pharma/Britnall, As- } \\
\text { traZeneca, Boehringer Ingelheim, Chiesi, Decision Resource, Dunn Group, Easton Associates, Gerson, } \\
\text { GlaxoSmithKline, Roche, Theravance, Almirall, CSL Behring, MedImmune, Novartis, Pearl, Takeda, For- } \\
\text { est, CME Incite, Novis, PriMed, Takeda, grants from AstraZeneca, Novartis, Otsuka, Boehringer Ingel- } \\
\text { heim, GlaxoSmithKline, and Johnson \& Johnson, outside the submitted work. Dr. Russ, Dr. McRae, Ms. } \\
\text { Treadow, Dr. Yu, Dr. Dutro, and Dr. Park are employees and stock holders of Pfizer Inc." }\end{array}$ \\
\hline
\end{tabular}

Notes Relevant comparisons: 1) Reduction versus reduction (pharmacological support)

\title{
Risk of bias
}

\begin{tabular}{|c|c|c|}
\hline $\begin{array}{l}\text { Blinding of outcome as- } \\
\text { sessment (detection bias) } \\
\text { All outcomes }\end{array}$ & Low risk & Abstinence was biochemically validated \\
\hline $\begin{array}{l}\text { Incomplete outcome data } \\
\text { (attrition bias) } \\
\text { All outcomes }\end{array}$ & Low risk & $\begin{array}{l}201 / 760(26.5 \%) \text { in the intervention group and } 234 / 750(31.2 \%) \text { in the control } \\
\text { group were lost to follow-up. Attrition rates were under } 50 \% \text { and similar be- } \\
\text { tween groups }\end{array}$ \\
\hline
\end{tabular}

\begin{tabular}{lll}
\hline Bias & Authors' judgement & Support for judgement \\
\hline $\begin{array}{l}\text { Random sequence genera- } \\
\text { tion (selection bias) }\end{array}$ & Low risk & Quote: "computer-generated block randomization schedule within site" \\
\hline $\begin{array}{l}\text { Allocation concealment } \\
\text { (selection bias) }\end{array}$ & Low risk & $\begin{array}{l}\text { Quote: "Investigators obtained participant identification numbers and treat- } \\
\text { ment group assignments through a web-based or telephone call-in drug man- } \\
\text { agement system. Participants, investigators, and research personnel were } \\
\text { blinded to randomization until after the database was locked" }\end{array}$ \\
\hline $\begin{array}{l}\text { Blinding of participants } \\
\text { and personnel (perfor- } \\
\text { mance bias) }\end{array}$ & Low risk & $\begin{array}{l}\text { Quote: "Randomized, blinded, placebo-controlled, multinational clinical trial"; } \\
\text { "Participants, investigators, and research personnel were blinded to random- } \\
\text { ization until after the database was locked"; "Participants started with a rec- } \\
\text { ommended varenicline (or matching placebo) dosage of } 0.5 \text { mg once daily for } \\
\text { 3 days, increasing to } 0.5 \text { mg twice daily for days } 4 \text { to } 7, \text { and then to the mainte- } \\
\text { nance dose of } 1 \text { mg twice daily" }\end{array}$
\end{tabular}

\section{Etter 2002}

Methods

\author{
Study design: RCT \\ Location: Switzerland \\ Setting: remote (mailings)
}


Etter 2002 (Continued)

Recruitment: physicians were invited to enrol their patients in the study; advertisements were placed in newspapers; a mail invitation was sent to a random sample of adult residents

Participants
Specialist population?: no
Participant characteristics: $477 / 923(52 \%)$ female; average age: 42.6 y; average cig/day: $30 ;$ nicotine de
pendence: FTND 6.1
Preference for quitting abruptly versus gradually: not reported

All participants received an information booklet covering reasons to reduce cigarette consumption, advice on how to reduce and addresses of smoking cessation clinics.

Comparator 1: Information booklet only: information booklet described above with no pharmacotherapy

Modality of support: mailed self-help. "The investigators had no in-person contact with participants and only minimal (reactive) telephone contact"

Overall contact time:n/a

Number of sessions: $\mathrm{n} / \mathrm{a}$

Pharmacotherapy: none

Quit date set?: no

Comparator 2: Placebo nicotine replacement group: information booklet plus NRT.

Modality of support: mailed self-help. "The investigators had no in-person contact with participants and only minimal (reactive) telephone contact"

Overall contact time: $\mathrm{n} / \mathrm{a}$

Number of sessions: $\mathrm{n} / \mathrm{a}$

Pharmacotherapy: placebo NRT: participants could choose between placebo patch (contains $25 \mathrm{mg}$ and delivers $15 \mathrm{mg}$ nicotine over 16 hours), placebo gum (contains $4 \mathrm{mg}$ and delivers $2 \mathrm{mg}$ nicotine), and placebo inhaler (a plug contains $10 \mathrm{mg}$ and delivers $5 \mathrm{mg}$ nicotine), or a combination of these

Quit date set?: no

Intervention: Nicotine replacement: information booklet plus NRT. Participants could choose between nicotine patch (contains $25 \mathrm{mg}$ and delivers $15 \mathrm{mg}$ nicotine over 16 hours), nicotine gum (contains $4 \mathrm{mg}$ and delivers $2 \mathrm{mg}$ nicotine), and nicotine inhaler (a plug contains $10 \mathrm{mg}$ and delivers $5 \mathrm{mg}$ nicotine), or a combination of these

Modality of support: mailed self-help. "The investigators had no in-person contact with participants and only minimal (reactive) telephone contact"

Overall contact time:n/a

Number of sessions:n/a

Pharmacotherapy: participants could choose between nicotine patch (contains $25 \mathrm{mg}$ and delivers 15 $\mathrm{mg}$ nicotine over 16 hours), nicotine gum (contains $4 \mathrm{mg}$ and delivers $2 \mathrm{mg}$ nicotine), and nicotine inhaler (a plug contains $10 \mathrm{mg}$ and delivers $5 \mathrm{mg}$ nicotine), or a combination of these

Quit date set?: no 
Etter 2002 (Continued)

Biochemical validation: none

Funding source

Swiss National Science Foundation to JFE (3233-054994.98 and 3200- 055141.98); Swiss Federal Office of Public Health. Nicotine and placebo products were provided by Pharmacia.

Author conflicts of interest JFE and JPZ received reimbursement from Pharmacia for attending international conferences. JFE was paid by Novartis for lectures. The Institute of Social and Preventive Medicine of the University of Geneva received financial support from Novartis to develop an education programme for users of nicotinell products. JPZ received research funds from Pharmacia

Notes Relevant comparisons: 1) Reduction method versus reduction method (pharmacological support)

\section{Risk of bias}

\begin{tabular}{|c|c|c|}
\hline Bias & Authors' judgement & Support for judgement \\
\hline $\begin{array}{l}\text { Random sequence genera- } \\
\text { tion (selection bias) }\end{array}$ & Low risk & $\begin{array}{l}\text { Quote: "Randomization was based on a computer-generated list of random } \\
\text { numbers" }\end{array}$ \\
\hline $\begin{array}{l}\text { Allocation concealment } \\
\text { (selection bias) }\end{array}$ & Unclear risk & $\begin{array}{l}\text { How the sequence was concealed is not reported. It appears that investigators } \\
\text { knew what they were sending to participants but there was minimal contact } \\
\text { with participants. However, it is unclear how much information investigators } \\
\text { had about participants }\end{array}$ \\
\hline $\begin{array}{l}\text { Blinding of participants } \\
\text { and personnel (perfor- } \\
\text { mance bias) } \\
\text { All outcomes }\end{array}$ & High risk & $\begin{array}{l}\text { Quote: "Nicotine and placebo products were sent to participants in unbrand- } \\
\text { ed packaging, similar in the two groups, labeled nicotine or placebo." Partic- } \\
\text { ipants were not aware of the nature of the products they received. Howev- } \\
\text { er, "The investigators were aware of the nature of products mailed to partici- } \\
\text { pants" Therefore, investigators were not blinded to intervention }\end{array}$ \\
\hline $\begin{array}{l}\text { Blinding of outcome as- } \\
\text { sessment (detection bias) } \\
\text { All outcomes }\end{array}$ & Low risk & $\begin{array}{l}\text { Abstinence was not biochemically confirmed, but contact was matched be- } \\
\text { tween groups }\end{array}$ \\
\hline $\begin{array}{l}\text { Incomplete outcome data } \\
\text { (attrition bias) } \\
\text { All outcomes }\end{array}$ & Low risk & $\begin{array}{l}100 / 265(38 \%) \text { in NRT group, } 135 / 269(50 \%) \text { in placebo group and } 175 / 389 \\
(45 \%) \text { in no-pharmacotherapy group were lost to follow-up at } 5 \text { years. There- } \\
\text { fore loss to follow-up was not more than } 50 \% \text { overall and comparable between } \\
\text { groups }\end{array}$ \\
\hline
\end{tabular}

Etter 2009

Study design: RCT
Location: Switzerland
Setting: remote (mailings)
Recruitment: advertisements on a smoking cessation website, in newspapers and through physicians
in private practice

Participants $\quad \mathrm{N}=314$

Specialist population?: no

Participant characteristics: 130/314 (41.3\%) female; average age: 43.1 y; average cig/day: 24; nicotine dependence: FTND 5.5 
Etter 2009 (Continued)

Preference for quitting abruptly versus gradually: not reported

Interventions

Comparator: Usual care (abrupt quitting): participants were instructed to quit abruptly on a target quit date, which was set for roughly 2 months after baseline questionnaire was completed

Modality of support: mail

Overall contact time: $\mathrm{n} / \mathrm{a}$

Number of sessions: $n / a$

Pharmacotherapy: $4 \mathrm{mg}$ nicotine gum for 8 weeks after the target quit date

Quit date set?: yes

Intervention: Precessation treatment (reduction): participants received a recommendation to decrease their cigarette consumption by half before quitting. Reduction took place over 4 weeks. No particular reduction method was specified

Modality of support: mail

Overall contact time: $\mathrm{n} / \mathrm{a}$

Number of sessions: $\mathrm{n} / \mathrm{a}$

Pharmacotherapy: $4 \mathrm{mg}$ nicotine gum for 4 weeks before and 8 weeks after target quit date

Quit date set?: yes

$\begin{array}{ll}\text { Outcomes } & \text { Definition of abstinence: } 1 \mathrm{~m} \text { point prevalence } \\ & \text { Longest follow-up: } 12 \mathrm{~m} \\ & \text { Biochemical validation: saliva cotinine and exhaled CO }\end{array}$

\begin{tabular}{ll} 
Funding source & Swiss National Science Foundation (3200-067835). Nicotine gum was provided at no charge by Pfizer \\
\hline Author conflicts of interest & $\begin{array}{l}\text { The Institute of Social and Preventive Medicine of the University of Geneva have received funding from } \\
\text { Novartis and Pfizer (both producers of nicotine products) to develop Internet-based smoking cessation } \\
\text { programmes for smokers (under the supervision of Dr Etter). Drs Etter and Cornuz have acted as advis- } \\
\text { ers to Pfizer, a manufacturer of smoking cessation medications }\end{array}$
\end{tabular}

Notes Relevant comparisons: 1) Reduction versus abrupt

\section{Risk of bias}

\begin{tabular}{lll}
\hline Bias & Authors' judgement & Support for judgement \\
\hline $\begin{array}{l}\text { Random sequence genera- } \\
\text { tion (selection bias) }\end{array}$ & Low risk & List of random numbers generated by a computer \\
\hline $\begin{array}{l}\text { Allocation concealment } \\
\text { (selection bias) }\end{array}$ & Low risk & $\begin{array}{l}\text { List of random numbers generated by a computer; a therapist was not in- } \\
\text { volved in delivering the intervention }\end{array}$ \\
\hline $\begin{array}{l}\text { Blinding of outcome as- } \\
\text { sessment (detection bias) } \\
\text { All outcomes }\end{array}$ & Low risk & Abstinence was biochemically validated \\
\hline $\begin{array}{l}\text { Incomplete outcome data } \\
\text { (attrition bias) } \\
\text { All outcomes }\end{array}$ & Low risk & $\begin{array}{l}12 \text { m survey completed by 89.0\% of precessation and } 87.5 \% \text { of usual care } \\
\text { group. Loss to follow-up low and similar across groups }\end{array}$ \\
\hline
\end{tabular}


Location: UK

Setting: community pharmacies

Recruitment: pharmacies recruited from areas of high smoking prevalence and pharmacists asked to recruit participants opportunistically as well as receiving referrals from general medical practitioners

Participants
\[ \begin{array}{l}\mathrm{N}=68 \\ \text { Specialist population?: no } \\ \text { Participant characteristics: } 34 / 68(50 \%) \text { female; average age: } 43.8 \text { y; average cig/day: } 19 ; \text { nicotine de- } \\ \text { pendence: FTND } 5.8\end{array} \]

Preference for quitting abruptly versus gradually: not reported fer to a smoking cessation programme at any point during reduction if they wanted to quit

Comparator 1: Short smoking reduction + in-person behavioural support: participants were asked to try to reduce cigarette consumption by $1 / 4$ (week 1 ), then by $1 / 2$ (week 2 ), then $3 / 4$ (week 3 ), to achieve abstinence in week 4 . Pharmacists explained the rationale for the programme by suggesting that learning a new pattern of smoking would prevent consumption increasing again by disrupting learned associations between cues and smoking behaviour. They encouraged participants to use NRT and choose lof 3 methods of reduction, called the "timer method", "smoke-free periods", or "unstructured". In the timer method, participants used a timer, such as a mobile phone, to signal when they could smoke and agreed to smoke only when the timer indicated it was appropriate to do so. This time lengthened on each occasion a person wanted to reduce. The smoke-free periods method divided the day into hours and participants progressively eliminated hours, agreeing not to smoke in the hours participants designated smoke-free. In the unstructured method participants were free to smoke when they liked but to set aside each day's cigarette ration in a pack. Pharmacists were encouraged to use either of the structured methods in preference, because there is evidence that they are more effective. Pharmacists enquired about participant's willingness to quit smoking at each visit and transferred a person ready to do so to a standard smoking cessation programme

Modality of support: face-to-face

Overall contact time: $1 \mathrm{~h} 20$ mins

Number of sessions: 8

Pharmacotherapy: nicotine patch and a short-acting form of NRT (2 mg gum, 2 mg sublingual tablets, 2 $\mathrm{mg}$ lozenge, inhalator or nasal spray). Participants were advised to continue using NRT for $9 \mathrm{~m}$, regardless of intention to reduce or stop, or failure to reduce or stop smoking, unless they had successfully quit

Quit date set?: yes

Comparator 2: Short smoking reduction + self-help booklet: pharmacists handed out written booklets detailing the same smoking reduction methods as described during the short reduction + behavioural support group (detailed above). They were asked to do this without any further advice or interaction. The booklet prompted participants to quit at regular intervals

Modality of support: self-help booklet

Overall contact time: $\mathrm{n} / \mathrm{a}$

Number of sessions: $\mathrm{n} / \mathrm{a}$ 
Pharmacotherapy: nicotine patch and a short-acting form of NRT ( 2 mg gum, 2 mg sublingual tablets, 2 mg lozenge, inhalator or nasal spray). Participants were advised to continue using NRT for $9 \mathrm{~m}$, regardless of intention to reduce or stop, or failure to reduce or stop smoking, unless they had successfully quit.

Quit date set?: yes

Intervention 1: Longer smoking reduction + in-person behavioural support: participants were asked to try to reduce smoking consumption by a $1 / 4$, then by $1 / 2$, then $3 / 4$ to achieve abstinence in 16 weeks. Pharmacists explained the rationale for the programme by suggesting that learning a new pattern of smoking would prevent consumption increasing again by disrupting learned associations between cues and smoking behaviour. They encouraged participants to use NRT and choose 1 of 3 methods of reduction, called the "timer method", "smoke-free periods", or "unstructured". In the timer method, participants used a timer, such as a mobile phone, to signal when they could smoke and agreed to smoke only when the timer indicated it was appropriate to do so. This time lengthened on each occasion a person wanted to reduce. The smoke-free periods method divided the day into hours and participants progressively eliminated hours, agreeing not to smoke in the hours participants designated smoke-free. In the unstructured method participants were free to smoke when they liked but to set aside each day's cigarette ration in a pack. Pharmacists were encouraged to use either of the structured methods in preference because there is evidence that they are more effective. Pharmacists enquired about participant's willingness to quit smoking at each visit and transferred a person ready to do so to a standard smoking cessation programme

Modality of support: face-to-face

Overall contact time: 1 h 20 mins

Number of sessions: 8

Pharmacotherapy: nicotine patch and a short-acting form of NRT: (2mg gum, $2 \mathrm{mg}$ sublingual tablets, $2 \mathrm{mg}$ lozenge, inhalator or nasal spray). Participants were advised to continue using NRT for $9 \mathrm{~m}$, regardless of intention to reduce or stop, or failure to reduce or stop smoking, unless they had successfully quit.

Quit date set?: yes

Intervention 2: Longer smoking reduction + self-help booklet: pharmacists handed out written booklets detailing the same smoking reduction methods as described during the longer reduction + behavioural support group (detailed above). They were asked to do this without any further advice or interaction. The booklet prompted participants to quit at regular intervals

Modality of support: self-help booklet

Overall contact time: $\mathrm{n} / \mathrm{a}$

Number of sessions: $\mathrm{n} / \mathrm{a}$

Pharmacotherapy: nicotine patch and a short-acting form of NRT (2 mg gum, 2 mg sublingual tablets, 2 mg lozenge, inhalator or nasal spray). Participants were advised to continue using NRT for $9 \mathrm{~m}$, regardless of intention to reduce or stop, or failure to reduce or stop smoking, unless they had successfully quit

Quit date set?: yes

Definition of abstinence: prolonged abstinence (abstinence or up to 5 cigarettes smoked only, from day 15 after quit day onwards)

Longest follow-up: $6 \mathrm{~m}$

Biochemical validation: exhaled CO concentration $<10 \mathrm{ppm}$

Funding source

National Prevention Research Initiative of the UK, administered by the MRC - funding partners are Alzheimer's Research UK, Alzheimer's Society, Biotechnology and Biological Sciences Research Council, British Heart Foundation, Cancer Research UK, Chief Scientist Office, Scottish Government Health Di- 
rectorate, Department of Health, Diabetes UK, Economic and Social Research Council, Engineering and Physical Sciences Research Council, Health and Social Care Research Division, Public Health Agency, Northern Ireland, Medical Research Council, Stroke Association, Wellcome Trust, Welsh Government, and the World Cancer Research Fund

\begin{tabular}{ll}
\hline Author conflicts of interest & "The authors declare that they have no competing interests" \\
\hline Notes & $\begin{array}{l}\text { Relevant comparisons: } 1 \text { ) Reduction method versus reduction method (self help/behavioural support; } \\
\text { shorter/longer) } \\
\text { Factorial RCT, but no interaction detected between arms }\end{array}$ \\
\hline
\end{tabular}

\section{Risk of bias}

\begin{tabular}{|c|c|c|}
\hline Bias & Authors' judgement & Support for judgement \\
\hline $\begin{array}{l}\text { Random sequence genera- } \\
\text { tion (selection bias) }\end{array}$ & Low risk & $\begin{array}{l}\text { Quote: "The research team generated the randomisation sequence using a } \\
\text { computer algorithm at www.randomization.com" }\end{array}$ \\
\hline \multirow[t]{2}{*}{$\begin{array}{l}\text { Allocation concealment } \\
\text { (selection bias) }\end{array}$} & Low risk & $\begin{array}{l}\text { Quote: "the allocations were given to pharmacists in numbered, sealed en- } \\
\text { velopes" }\end{array}$ \\
\hline & & Comment: These envelopes were opaque \\
\hline $\begin{array}{l}\text { Blinding of outcome as- } \\
\text { sessment (detection bias) } \\
\text { All outcomes }\end{array}$ & Low risk & Abstinence was biochemically validated \\
\hline $\begin{array}{l}\text { Incomplete outcome data } \\
\text { (attrition bias) } \\
\text { All outcomes }\end{array}$ & High risk & $\begin{array}{l}14 / 19(74 \%) \text { in the behavioural shorter-reduction group, } 14 / 17(82 \%) \text { in the be- } \\
\text { havioural longer-reduction group, } 13 / 15(87 \%) \text { in the self-help shorter-reduc- } \\
\text { tion group, and } 13 / 17(77 \%) \text { in the self-help longer-reduction group were lost } \\
\text { to follow-up. Attrition rates were high in all groups }\end{array}$ \\
\hline
\end{tabular}

Flaxman 1978

\begin{tabular}{|c|c|}
\hline \multirow[t]{4}{*}{ Methods } & Study design: RCT \\
\hline & Location: USA \\
\hline & Setting: community smoking cessation clinics \\
\hline & $\begin{array}{l}\text { Recruitment: public service announcements by a stop-smoking clinic on television, radio and in news- } \\
\text { papers }\end{array}$ \\
\hline \multirow[t]{4}{*}{ Participants } & $N=64$ \\
\hline & Specialist population?: no \\
\hline & $\begin{array}{l}\text { Participant characteristics: } 32 / 64(50 \%) \text { female; average age: not reported; average cig/day: } 26 \text {; nico- } \\
\text { tine dependence: } \text { mean years smoked }=20.3 \mathrm{y}\end{array}$ \\
\hline & Preference for quitting abruptly versus gradually: not reported \\
\hline Interventions & $\begin{array}{l}\text { Pre-quit participants met with the experimenter twice a week for } 1 / 2 \text {-h sessions (except in the imme- } \\
\text { diate quit condition). Self-control techniques presented to all participants included: the use of stimu- } \\
\text { lus change and the introduction of novel stimuli into daily activities, a } 1 / 2-h \text { tape of muscle relaxation, } \\
\text { thought-stopping activities, worry beads, a new hobby, public commitment procedure, writing letters, } \\
\text { self-reinforcement and empathic rehearsal of reasons to give up. The number of post-quit sessions } \\
\text { were dependent on the participants' progress }\end{array}$ \\
\hline
\end{tabular}


Comparator 1: Immediate quit: participants were scheduled to quit smoking and begin aversive conditioning or attention control sessions the next day

Modality of support: face-to-face

Overall contact time: unclear

Number of sessions: approx. 3

Pharmacotherapy: none

Quit date set?: yes

Comparator 2: Target date: a date approximately 2 weeks from the 1st session was selected for abrupt quitting

Modality of support: face-to-face

Overall contact time: $3 \mathrm{~h}$

Number of sessions: approx. 6

Pharmacotherapy: none

Quit date set?: yes

Intervention 1: Gradual: Gutmann \& Marston's stimulus hierarchy technique; situations leading to smoking were categorised and rank ordered according to anticipated difficulty of not smoking in each. Participants were instructed to give up in the easiest situation first, progressing to the hardest, adding 1 situation every 3 days

Modality of support: face-to-face

Overall contact time: $3 \mathrm{~h}$

Number of sessions: approx. 6

Pharmacotherapy: none

Quit date set?: no

Intervention 2: Partially gradual: same as 'gradual' group above, but participants quit abruptly when their smoking rates dropped to half of baseline

Modality of support: face-to-face

Overall contact time: $4 \mathrm{~h}$

Number of sessions: approx. 8

Pharmacotherapy: none

Quit date set?: no

Outcomes

Definition of abstinence: unclear

Longest follow-up: $6 \mathrm{~m}$

Biochemical validation: no

\section{Funding source}

Mental Health Grant No. MH20751

Author conflicts of interest not reported 
Flaxman 1978 (Continued)

Notes

Relevant comparisons: 1) Reduction versus abrupt 2) Reduction method versus reduction method (gradual/partially gradual)

\section{Risk of bias}

\begin{tabular}{lll}
\hline Bias & Authors' judgement & Support for judgement \\
\hline $\begin{array}{l}\text { Random sequence genera- } \\
\text { tion (selection bias) }\end{array}$ & Unclear risk & Not reported \\
\hline $\begin{array}{l}\text { Allocation concealment } \\
\text { (selection bias) }\end{array}$ & Unclear risk & Not reported \\
\hline $\begin{array}{l}\text { Blinding of outcome as- } \\
\text { sessment (detection bias) }\end{array}$ & High risk & $\begin{array}{l}\text { Abstinence was not biochemically validated and there was difference in con- } \\
\text { All outcomes }\end{array}$ \\
\hline $\begin{array}{l}\text { Incomplete outcome data } \\
\text { (attrition bias) } \\
\text { All outcomes }\end{array}$ & Low risk & Quote: "Follow-up data were obtained from all subjects" \\
\hline
\end{tabular}

\section{Garcia 2000}

\begin{tabular}{ll}
\hline Methods & Study design: RCT \\
Location: Spain \\
Setting: community \\
Recruitment: press and radio advertisements \\
\hline
\end{tabular}

Participants $\quad \mathrm{N}=162$

Specialist population?: no

Participant characteristics: 84/162 (52\%) female; average age: 32.2 y; average cig/day: 26; nicotine dependence: FTND 7.2

Preference for quitting abruptly versus gradually: not reported

All participants except those in the 2 comparator arms received the following multicomponent intervention: (a) a motivational contract - a refundable guarantee was deposited at the beginning of treatment; (b) self-monitoring of smoking behaviour was performed before and during treatment; (c) information on smoking was provided; (d) stimulus control was implemented. Participants had to follow some rules to reduce cigarette consumption (not smoking the final third of the cigarette, not accepting cigarettes offered by other people, taking fewer drags on each cigarette, not smoking during a progressive number of situations); (e) nicotine fading and cigarette fading; (f) physiological feedback (expired $\mathrm{CO}$ ); (g) strategies to prevent relapse and progressive self-control of smoking behaviour were emphasised as being essential for final success in giving up smoking. All participants were asked to abstain from smoking 24 hours before the last treatment day, although they could try to stop before if they wished to.

Comparator: no treatment (not eligible for analyses as this group was not randomised): participants attended an information session but did not receive any treatment sessions

Modality of support: $n / a$

Overall contact time: $\mathrm{n} / \mathrm{a}$ 


\section{Number of sessions: $n / a$ \\ Pharmacotherapy: none \\ Quit date set?: $\mathrm{n} / \mathrm{a}$}

Intervention 1: 10-session multicomponent package: participants received the multicomponent programme above. The intervention was delivered over 10 group sessions - 2 a week for 5 weeks

Modality of support: face-to-face

Overall contact time: $10 \mathrm{~h}$

Number of sessions: 10

Pharmacotherapy: no

Quit date set?: yes

Intervention 2: 5-session multicomponent package: participants received the multicomponent programme above. The intervention was delivered over 5 group sessions - 1 a week for 5 weeks

Modality of support: face-to-face

Overall contact time: $5 \mathrm{~h}$

Number of sessions: 5

Pharmacotherapy: none

Quit date set?: yes

Intervention 3: 5-session multicomponent package + a 125-page self-help manual: multicomponent package as above provided over 5 group sessions ( 1 a week for 5 weeks). The self-help manual consisted of nicotine fading and some behavioural techniques. Participants were asked to read and complete 1 of the 5 units in the manual corresponding to a certain session before that particular session took place. The manual aimed to lead to complete cessation in the 4th week of the 5-week programme, with option to quit sooner if they wanted to. It had 5 units, including exercises such as listing reasons for not smoking and for smoking

Modality of support: face-to-face + self-help manual

Overall contact time: $5 \mathrm{~h}$

Number of sessions: 5

Pharmacotherapy: none

Quit date set?: yes

Intervention 4: self-help manual only: a 125-page self-help manual. The manual was designed to lead to complete cessation in the 4th week of the 5-week programme, with an option to quit sooner. The manual was made up of 5 units. Each unit was designed to correspond with 1 of the 5 treatment weeks in the above multicomponent programme. The techniques in Ihe manual were the same as the techniques in the multicomponent programme and included specific exercises

Modality of support: self-help manual

Overall contact time: $\mathrm{n} / \mathrm{a}$

Number of sessions: $\mathrm{n} / \mathrm{a}$

Pharmacotherapy: none

Quit date set?: yes 
Garcia 2000 (Continued)

Outcomes

Definition of abstinence: 7-day point prevalence

Longest follow-up: $12 \mathrm{~m}$

Biochemical validation: expired CO

Funding source

The Dirección General de Investigación Científica y Técnica (DGICYT) of the Ministry of Education and Science, Spain

Author conflicts of interest Not reported

Notes Relevant comparisons: Reduction method versus reduction method (modality; intensity)

\section{Risk of bias}

\begin{tabular}{lll}
\hline Bias & Authors' judgement & Support for judgement \\
\hline $\begin{array}{l}\text { Random sequence genera- } \\
\text { tion (selection bias) }\end{array}$ & Unclear risk & Not reported \\
\hline $\begin{array}{l}\text { Allocation concealment } \\
\text { (selection bias) }\end{array}$ & Unclear risk & Not reported \\
\hline $\begin{array}{l}\text { Blinding of outcome as- } \\
\text { sessment (detection bias) } \\
\text { All outcomes }\end{array}$ & Low risk & Abstinence was biochemically validated \\
\hline $\begin{array}{l}\text { Incomplete outcome data } \\
\text { (attrition bias) } \\
\text { All outcomes }\end{array}$ & Unclear risk & Not reported \\
\hline
\end{tabular}

Gariti 2004

\begin{tabular}{ll}
\hline Methods & Study design: RCT \\
Location: USA \\
Setting: University \\
Recruitment: University campus notices, local newspaper advertisements, community posters, bul- \\
letins to healthcare providers, the internet, 'word of mouth' and candidates who did not meet eligibility \\
criteria for another smoking cessation study
\end{tabular}


Gariti 2004 (Continued)

cigarette tapering, as well as feelings and problems about becoming smoke-free. Session 6: took place at completion of the drops and assessed the treatment method and discussed relapse prevention.

Comparator: Placebo drops + manualised counselling

Modality of support: face-to-face

Overall contact time: 3 h 30 mins

Number of sessions: 6

Pharmacotherapy: placebo Accu Drop/Take Out/NicoBloc

Quit date set?: yes

Intervention: Active drops + manualised counselling

Modality of support: face-to-face

Overall contact time: 3 h 30 mins

Number of sessions: 6

Pharmacotherapy: Accu Drop/Take Out/NicoBloc: FDA-approved corn syrup-based food additive that is applied to the filter of a cigarette. The applied drops form an occlusive barrier that traps nicotine and tar that ordinarily would be ingested during regular cigarette smoking

Quit date set?: yes

Definition of abstinence: 7-day point prevalence
Longest follow-up: $6 \mathrm{~m}$
Biochemical validation: exhaled $\mathrm{CO}$ and urinary cotinine

Funding source The National Institute on Drug Abuse

Author conflicts of interest Not reported

Notes $\quad$ Relevant comparisons: 1) Reduction method versus reduction method (Accu Drop)

\section{Risk of bias}

\begin{tabular}{lll}
\hline Bias & Authors' judgement & Support for judgement \\
\hline $\begin{array}{l}\text { Random sequence genera- } \\
\text { tion (selection bias) }\end{array}$ & Unclear risk & Not reported \\
\hline $\begin{array}{l}\text { Allocation concealment } \\
\text { (selection bias) }\end{array}$ & Low risk & $\begin{array}{l}\text { Quote: "The 'medication' blinding was maintained by the pharmacist and } \\
\text { could only be broken in the event of a life-threatening emergency or where in- } \\
\text { formation about the drop type was critical for medical care (e.g., in the event } \\
\text { of an allergic response)." }\end{array}$ \\
\hline
\end{tabular}

Blinding of outcome as- Low risk Abstinence was biochemically validated

sessment (detection bias)

All outcomes

Incomplete outcome data Low risk Overall dropout was $45 \%$ and this did not differ between groups
(attrition bias)

All outcomes 


\begin{tabular}{|c|c|}
\hline \multirow[t]{4}{*}{ Methods } & Study design: RCT \\
\hline & Location: Spain \\
\hline & Setting: University \\
\hline & $\begin{array}{l}\text { Recruitment: announcements made across academic centres of the University and through word of } \\
\text { mouth }\end{array}$ \\
\hline \multirow[t]{4}{*}{ Participants } & $\mathrm{N}=24$ in total (14 in 2 eligible trial arms only) \\
\hline & Specialist population?: no \\
\hline & $\begin{array}{l}\text { Participant characteristics: } 11 / 24 \text { (46\%) female; average age: } 32.7 \text { y; average cig/day: } 20 \text {; nicotine de- } \\
\text { pendence: not reported }\end{array}$ \\
\hline & Preference for quitting abruptly versus gradually: not reported \\
\hline \multirow[t]{13}{*}{ Interventions } & $\begin{array}{l}\text { All participants were given the same instructions on how to avoid lapses. Contingency payments were } \\
\text { given to participants when their goals were met and aversive contingency payments were made to an } \\
\text { organisation disliked by the participant when goals were not met }\end{array}$ \\
\hline & $\begin{array}{l}\text { Comparator: Abrupt quitting with abstinence goal (A1): participants received instructions to stop smok- } \\
\text { ing completely on the first day of treatment }\end{array}$ \\
\hline & $\begin{array}{l}\text { Modality of support: } 1 \text { face-to-face session at beginning of treatment, then participants mailed ciga- } \\
\text { rette counts back }\end{array}$ \\
\hline & Overall contact time: unclear \\
\hline & Number of sessions: 1 \\
\hline & Pharmacotherapy: none \\
\hline & Quit date set?:yes \\
\hline & $\begin{array}{l}\text { Intervention: Reduction with abstinence goal (A2): participants received instructions to reduce their } \\
\text { cigarette consumption over } 4 \text { weeks ( } 25 \% \text { off baseline week 1,50\% off baseline week 2, } 75 \% \text { off base- } \\
\text { line week 3, abstinence week } 4 \text { ) }\end{array}$ \\
\hline & $\begin{array}{l}\text { Modality of support: } 1 \text { face-to-face session at beginning of treatment, then participants mailed ciga- } \\
\text { rette counts back }\end{array}$ \\
\hline & Overall contact time: unclear \\
\hline & Number of sessions: 1 \\
\hline & Pharmacotherapy: none \\
\hline & Quit date set?: yes \\
\hline
\end{tabular}

Outcomes Definition of abstinence: 5-day point prevalence

Longest follow-up: $12 \mathrm{~m}$

Biochemical validation: no

\begin{tabular}{ll}
\hline Funding source & Not reported \\
\hline Author conflicts of interest & Not reported \\
\hline Notes & Relevant comparisons: Reduction versus abrupt \\
\hline
\end{tabular}

Smoking reduction interventions for smoking cessation (Review) 
Gil Roales-Nieto 1992a (Continued)

There were 2 additional trial arms (not detailed above), but these are not eligible for inclusion in this review as the goal of the intervention was not abstinence

\section{Risk of bias}

\begin{tabular}{lll}
\hline Bias & Authors' judgement & Support for judgement \\
\hline $\begin{array}{l}\text { Random sequence genera- } \\
\text { tion (selection bias) }\end{array}$ & Unclear risk & Not reported \\
\hline $\begin{array}{l}\text { Allocation concealment } \\
\text { (selection bias) }\end{array}$ & Unclear risk & Not reported \\
\hline $\begin{array}{l}\text { Blinding of outcome as- } \\
\text { sessment (detection bias) } \\
\text { All outcomes }\end{array}$ & Low risk & $\begin{array}{l}\text { Abstinence was not validated; the amount of contact with the research team } \\
\text { was low and similar between study arms }\end{array}$ \\
\hline $\begin{array}{l}\text { Incomplete outcome data } \\
\text { (attrition bias) }\end{array}$ & Low risk & $\begin{array}{l}\text { 1 participant, assigned to A1 (abrupt quitting) resigned from abstinence goal. } \\
\text { As a result 14\% dropped out in the abrupt group and 0\% in the reduction } \\
\text { group, i.e. low, and similar between groups }\end{array}$ \\
\hline
\end{tabular}

\section{Glasgow 1978}

Methods Study design: RCT

Location: USA

Setting: unclear

Recruitment: local media announcements

Participants
$\begin{aligned} & \mathrm{N}=62 \\ & \text { Specialist population?: no } \\ & \text { Participant characteristics: } 30 / 62 \text { (48\%) female; average age: } 32.6 \text { y; average cig/day: } 31 \text {; nicotine de- } \\ & \text { pendence: not reported }\end{aligned}$

Preference for quitting abruptly versus gradually: not reported

Comparator 1: High-contact rapid smoking: the procedure and spacing of rapid smoking were identical to that used in the intervention groups below, but all sessions were therapist-administered. There was a 9-day "preparation period" after an initial meeting for participants in this group before beginning rapid smoking. This was to ensure that all groups completed treatment at the same time

Modality of support: face-to-face

Overall contact time: 2 h 50 mins

Number of sessions: 7

Pharmacotherapy: none

Quit date set?: no

Comparator 2: High-contact normal-paced smoking: participants received an "aversive smoking" procedure. Participants smoked at their normal rate while focusing on the unpleasant aspects of the pure smoking experience. Participants were instructed to smoke until they could not bear to continue or until 5 minutes elapsed. If they smoked faster than 1 puff every 15 - 20 secs, they were reminded to smoke at their normal rate 
Overall contact time: 2 h 30 mins

Number of sessions: 7

Pharmacotherapy: none

Quit date set?: no

Intervention 1: Minimal contact self-control: 37-page manual detailing a multicomponent smoking cessation programme. Initial chapters focused on training in progressive relaxation as a coping strategy and stimulus control techniques for hierarchical reduction. A rapid-smoking procedure also took place, with 6 sessions consisting of 2 trials of rapid smoking each. Participants met with a therapist to receive their manual, a rationale for the programme, and a demonstration of relaxation procedures. They then worked on their own, meeting once more with their therapist midway through the programme to receive their first rapid-smoking session. Subsequent rapid-smoking and relaxation sessions were selfadministered by participants at home. Therapists called weekly to check on progress and to answer questions

Modality of support: self-help manual

Overall contact time: 1 h 30 mins

Number of sessions: 5 (2 face-to-face, 3 telephone)

Pharmacotherapy: none

Quit date set?: no

Intervention 2: High-contact self-control: participants received the same manual as the minimal contact group but had regular meetings with a therapist. Participants were assigned to read a section of the manual and then met with their therapist to implement the assignments in that section. 7 meetings were held over the 3-week treatment period. Participants received more direction from therapists on relaxation and stimulus control procedures than minimal-contact participants, but rapid-smoking and relaxation sessions were held at home after initial demonstrations. Treatment techniques and the sequence of components were identical to those of the minimal-contact group

Modality of support: face-to-face, self-help manual

Overall contact time: $3 \mathrm{~h}$

Number of sessions: 7

Pharmacotherapy: none

Quit date set?: no

Definition of abstinence: point prevalence (no further information given)
Longest follow-up: $6 \mathrm{~m}$
Biochemical validation: not at $6 \mathrm{~m}$ follow-up (only at $3 \mathrm{~m}$ )

Funding source Not reported

Author conflicts of interest Not reported

Notes

Relevant comparisons: 1) Reduction method versus reduction method (modality). However, we were unable to include this in any meta-analyses as the abstinence data are not presented by study groups. We contacted the authors, but they were unable to supply the data due to the age of the study

\section{Risk of bias}


Glasgow 1978 (Continued)

\section{Bias Authors' judgement Support for judgement}

Random sequence genera- Unclear risk tion (selection bias)

Quote: "Interested subjects were then randomly assigned to one of four treatment groups."

Comment: No further information provided

\begin{tabular}{|c|c|c|}
\hline $\begin{array}{l}\text { Allocation concealment } \\
\text { (selection bias) }\end{array}$ & Unclear risk & $\begin{array}{l}\text { Quote: "Interested subjects were then randomly assigned to one of four treat- } \\
\text { ment groups." }\end{array}$ \\
\hline
\end{tabular}

Comment: No further information provided

\begin{tabular}{lll}
\hline Blinding of outcome as- & High risk & $\begin{array}{l}\text { Self-reported abstinence rates and different levels of contact between study } \\
\text { sessment (detection bias) }\end{array}$ \\
All outcomes & arms (biochemical validation took place at $3 \mathrm{~m}$ but not $6 \mathrm{~m}$ )
\end{tabular}

All outcomes

\begin{tabular}{lll}
\hline $\begin{array}{l}\text { Incomplete outcome data } \\
\text { (attrition bias) } \\
\text { All outcomes }\end{array}$ & Unclear risk & $50 / 62(81 \%)$ were available after $6 \mathrm{~m}$, but this is not reported by study arm \\
\hline Other bias & Unclear risk & $\begin{array}{l}\text { Long-term abstinence was measured but has not been reported split by study } \\
\text { group. Insufficient information to judge whether this is as a result of selective } \\
\text { reporting }\end{array}$ \\
\hline
\end{tabular}

\section{Glasgow 1989}

\begin{tabular}{|c|c|}
\hline \multirow[t]{4}{*}{ Methods } & Study design: cluster-RCT \\
\hline & Location: USA \\
\hline & Setting: unclear \\
\hline & $\begin{array}{l}\text { Recruitment: "via flyers, newspaper advertisements, and public service announcements on local radio } \\
\text { stations" }\end{array}$ \\
\hline \multirow[t]{4}{*}{ Participants } & $N=66$ \\
\hline & Specialist population?: no \\
\hline & $\begin{array}{l}\text { Participant characteristics: } 37 / 66 \text { (56\%) female; average age: } 39.6 \text { y; average cig/day: } 26 \text {; nicotine de- } \\
\text { pendence: FTND } 6.8\end{array}$ \\
\hline & Preference for quitting abruptly versus gradually: not reported \\
\hline \multirow[t]{6}{*}{ Interventions } & $\begin{array}{l}\text { All participants attended } 6 \text { weekly meetings, carried out nicotine fading and used a booklet to record } \\
\text { the brand, number, and amount of cigarettes smoked }\end{array}$ \\
\hline & $\begin{array}{l}\text { Comparator: Abstinence-based condition: explicitly stated quit date (4th session) and that the first } 3 \\
\text { sessions would prepare them to quit and the last } 3 \text { to stay quit. Participants targeted individual cig- } \\
\text { arettes to eliminate, with the goal of gaining experience coping with nonsmoking in particular situa- } \\
\text { tions, not rate reduction, so specific reduction goals were not provided. Post-quit sessions emphasised } \\
\text { relapse prevention }\end{array}$ \\
\hline & Modality of support: face-to-face \\
\hline & Overall contact time: unclear \\
\hline & Number of sessions: 6 \\
\hline & Pharmacotherapy: none \\
\hline
\end{tabular}


Glasgow 1989 (Continued)

\section{Quit date set?: yes}

Intervention: Cessation-controlled smoking: participants were encouraged to stop smoking entirely but could also choose to remain smoking at a controlled rate. They were asked to change brands to 1 with nicotine levels 33\% - 50\% of baseline levels; participants were assisted to select strategies to reduce number of cigarettes smoked per day to $50 \%-67 \%$ of baseline, then $33 \%-50 \%$ of baseline. Participants were then given the choice of quitting (recommended option) or of making further changes in smoking topography, but continuing to smoke at reduced levels. Participants choosing to quit set quit dates, and those choosing the controlled smoking option targeted a reduction in the percent of each cigarette smoked to $33 \%-50 \%$ of baseline levels

Modality of support: face-to-face

Overall contact time: unclear

Number of sessions: 6

Pharmacotherapy: none

Quit date set?: no (participants were given a choice to set a quit date or to continue to reduce)

\begin{tabular}{ll} 
Outcomes & $\begin{array}{l}\text { Definition of abstinence: 7-day point prevalence } \\
\text { Longest follow-up: } 6 \mathrm{~m} \\
\text { Biochemical validation: exhaled CO }\end{array}$ \\
\hline Funding source & The National Heart, Lung, and Blood Institute (grants \#HL29547 and \#HL33739) \\
\hline Author conflicts of interest & Not reported \\
\hline Notes & $\begin{array}{l}\text { Relevant comparisons: Reduction method versus reduction method (quit day and reduction focus) } \\
\text { Cluster-RCT as randomisation was performed on groups of participants rather than individual partici- } \\
\text { pants. Analysis does not account for an ICC, but as the 95\% Cl spans } 1 \text { any adjustment will only slightly } \\
\text { widen the Cl and have no impact on conclusions }\end{array}$
\end{tabular}

\section{Risk of bias}

\begin{tabular}{lll}
\hline Bias & Authors' judgement & Support for judgement \\
\hline $\begin{array}{l}\text { Random sequence genera- } \\
\text { tion (selection bias) }\end{array}$ & Unclear risk & Not reported \\
\hline $\begin{array}{l}\text { Allocation concealment } \\
\text { (selection bias) }\end{array}$ & Unclear risk & Not reported \\
\hline $\begin{array}{l}\text { Blinding of outcome as- } \\
\text { sessment (detection bias) }\end{array}$ & Low risk & Abstinence was biochemically validated \\
All outcomes & \\
\hline $\begin{array}{l}\text { Incomplete outcome data } \\
\text { (attrition bias) }\end{array}$ & Low risk & $\begin{array}{l}\text { Only 20\% of the participants failed to complete the assessments at } 6 \text { months, } \\
\text { with no appreciable difference between conditions }(8 / 31(26 \%) \text { and } 5 / 35(14 \%) \\
\text { lost to follow-up in abstinence-based and cessation-controlled groups respec- } \\
\text { tively) }\end{array}$ \\
\hline
\end{tabular}

Glasgow 2009a

Methods Study design: RCT


Glasgow 2009a (Continued)

Location: USA

Setting: healthcare clinic and telephone

Recruitment: "Members of the Kaiser Permanente - Health Maintenance Organisation eligible for the programme were notified by letter and provided with an "opt out" postcard. Members who did not decline were contacted."

\begin{tabular}{ll}
\hline Participants & N $=391$ \\
Specialist population?: no, although most had multiple chronic conditions \\
Participant characteristics: $232 / 391$ (73\%) female; average age: 55.4 y; average cig/day: $21 ;$ nicotine de- \\
pendence: not reported \\
Preference for quitting abruptly versus gradually: not reported \\
Comparator: Enhanced usual care: participants took part in in a recruitment/baseline call. 3 quarterly, \\
generic health-education mailings were sent out \\
Modality of support: newsletters \\
Overall contact time: $n /$ a \\
Number of sessions: $n / a$ \\
Pharmacotherapy: none \\
Quit date set?: no \\
Intervention: Smoking reduction: telephone counselling calls aimed to increase participant self-effica- \\
cy to achieve and sustain reduced smoking levels (using a graduated reduction approach). Participants \\
were encouraged to set an initial goal of a 1/3 reduction in number of cigarettes smoked. Based upon \\
progress and self-efficacy, participants' later reduction goals were individually tailored, although they \\
were encouraged to attempt at least a $50 \%$ reduction. Participants who achieved a $50 \%$ or greater re- \\
duction were encouraged to quit. Newsletters were sent out including tailoring based upon data col- \\
lected during the counselling calls
\end{tabular}

Modality of support: telephone and newsletter

Overall contact time: unclear

Number of sessions: 4

Pharmacotherapy: none

Quit date set?: no

Outcomes

Definition of abstinence: point prevalence

Longest follow-up: $12 \mathrm{~m}$

Biochemical validation:expired CO

\begin{tabular}{ll}
\hline Funding source & National Cancer Institute (RO1 CA 90974-01) \\
\hline Author conflicts of interest & Not reported \\
\hline Notes & $\begin{array}{l}\text { Relevant comparisons: 1) Reduction versus no treatment, but content of comparator arm is unclear - } \\
\text { described as 'generic health education mailings'; unclear whether this included any specific advice to } \\
\text { stop smoking }\end{array}$
\end{tabular}

\section{Risk of bias}


Glasgow 2009a (Continued)

\begin{tabular}{|c|c|c|}
\hline Bias & Authors' judgement & Support for judgement \\
\hline $\begin{array}{l}\text { Random sequence genera- } \\
\text { tion (selection bias) }\end{array}$ & Low risk & $\begin{array}{l}\text { Quote: "randomly assigned to intervention conditions, using a computer algo- } \\
\text { rithm developed by the project statistician." }\end{array}$ \\
\hline $\begin{array}{l}\text { Allocation concealment } \\
\text { (selection bias) }\end{array}$ & Unclear risk & Not reported \\
\hline $\begin{array}{l}\text { Blinding of outcome as- } \\
\text { sessment (detection bias) } \\
\text { All outcomes }\end{array}$ & Low risk & Abstinence was biochemically validated \\
\hline $\begin{array}{l}\text { Incomplete outcome data } \\
\text { (attrition bias) } \\
\text { All outcomes }\end{array}$ & Low risk & $\begin{array}{l}97 / 200(49 \%) \text { and } 63 / 191(33 \%) \text { were lost to follow-up in the smoking-reduc- } \\
\text { tion and usual-care arms respectively }\end{array}$ \\
\hline
\end{tabular}

Gunther 1992

Study design: RCT
Location: Austria
Setting: smoker's counselling service - part of the Innsbruck University Hospital of Psychiatry
Recruitment: patients consulting a smoker's counselling centre were recruited

\begin{tabular}{|c|c|}
\hline \multirow[t]{4}{*}{ Participants } & $N=110$ \\
\hline & Specialist population?: no \\
\hline & $\begin{array}{l}\text { Participant characteristics: gender not reported; average age: not reported; average cig/day: } 27 \text {; nico- } \\
\text { tine dependence: } 20 \text { y of smoking }\end{array}$ \\
\hline & Preference for quitting abruptly versus gradually: not reported \\
\hline
\end{tabular}

Interventions All participants were taught techniques of behaviour therapy and of cognitive self-control. Successful participants in both groups had 3 additional booster sessions ( 1 a month for 3 months)

Comparator: Abrupt: In the first 5 sessions/weeks after history-taking participants were taught techniques and set a quit date. The remaining 5 sessions after quitting were used for follow-up treatment

Modality of support: face-to-face

Overall contact time: $12 \mathrm{~h}$

Number of sessions: 12

Pharmacotherapy: none

Quit date set?: yes

Intervention: Reduction/gradual: from the 2 nd session/week of counselling the number of cigarettes was reduced in the gradual-withdrawal arm. Depending on initial consumption the number of cigarettes per week was reduced by 5 - 10 cigarettes. It is unclear over how long reduction took place

Modality of support: face-to-face

Overall contact time: $12 \mathrm{~h}$

Number of sessions: 12 
Gunther 1992 (Continued)

Pharmacotherapy: none

Quit date set?: unclear

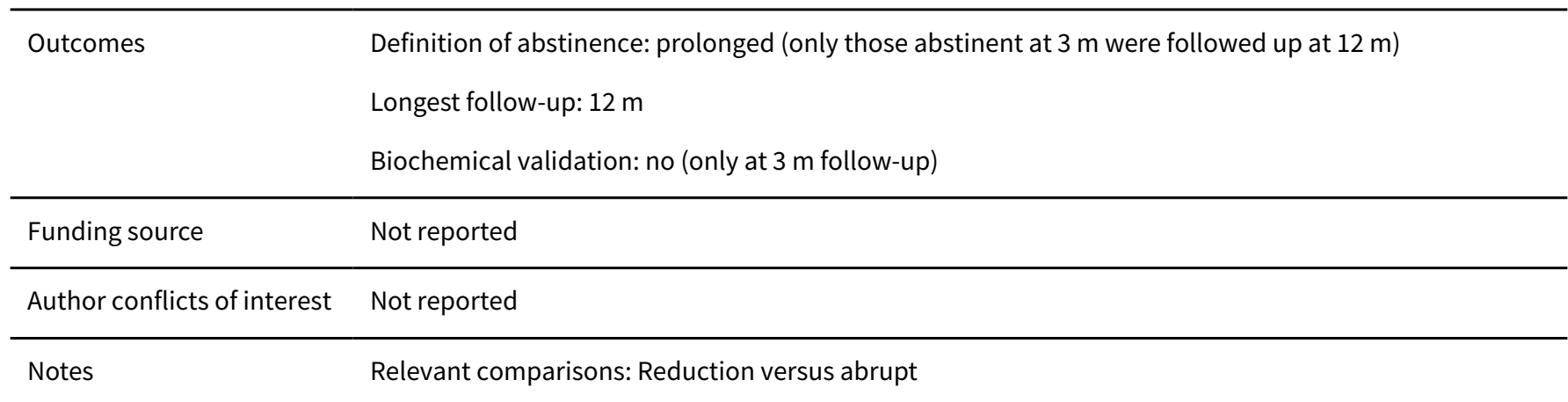

\section{Risk of bias}

\begin{tabular}{lll}
\hline Bias & Authors' judgement & Support for judgement \\
\hline $\begin{array}{l}\text { Random sequence genera- } \\
\text { tion (selection bias) }\end{array}$ & Low risk & Quote: "Computer generated randomisation list" \\
\hline $\begin{array}{l}\text { Allocation concealment } \\
\text { (selection bias) }\end{array}$ & Unclear risk & Not reported \\
\hline $\begin{array}{l}\text { Blinding of outcome as- } \\
\text { sessment (detection bias) } \\
\text { All outcomes }\end{array}$ & Low risk & $\begin{array}{l}\text { Abstinence rates were not biochemically verified at } 12 \text { m, but the amount of } \\
\text { contact was similar between groups, meaning that misreporting of abstinence } \\
\text { was likely to be balanced between groups }\end{array}$ \\
\hline $\begin{array}{l}\text { Incomplete outcome data } \\
\text { (attrition bias) }\end{array}$ & Low risk & $\begin{array}{l}\text { Only initially abstinent smokers were followed up at } 1 \text { year as investigators } \\
\text { were measuring prolonged abstinence, i.e. } 42 \text { of abrupt and } 40 \text { of withdrawal. } \\
\text { Of these, response rates were similar between groups } 27 / 42=64 \% \text { and } 31 / 40= \\
78 \%\end{array}$ \\
\hline
\end{tabular}

\section{Hanson 2008}

\begin{tabular}{ll}
\hline Methods & Study design: RCT \\
Location: USA & Setting: University \\
& Recruitment: from 14 traditional and alternative high schools \\
\hline Participants & Specialist population?: young people aged 13 - 19 \\
& $\begin{array}{l}\text { Participant characteristics: } 60 / 103 \text { (\%) female; average age: } 16.6 \text { y; average cig/day: } 12 ; \text { nicotine depen- } \\
\text { dence: not reported }\end{array}$ \\
& Preference for quitting abruptly versus gradually: not reported \\
\hline All participants received CBT to reduce smoking. They were instructed to reduce by $25 \%$ of baseline \\
smoking during the first week and $50 \%$ over the next 3 weeks. At week 4 participants were asked "if \\
they wanted to set a quit date within one week." Those who set a quit date received 4 additional weeks \\
of their choice of medication and CBT sessions designed to help them to quit
\end{tabular}


Modality of support: face-to-face

Overall contact time: minimum $2 \mathrm{~h} 30$ mins, maximum $4 \mathrm{~h} 10$ mins

Number of sessions: minimum 6 (plus a further 4 if went forward to quit)

Pharmacotherapy: Placebo was in the form of $400 \mathrm{mg}$ folic acid pills, and therefore was not matched to the intervention NRT

Quit date set?: yes

Intervention 1: Nicotine patch + smoking reduction counselling, as described above

Modality of support: face-to-face

Overall contact time: minimum $2 \mathrm{~h} 30$ mins, maximum $4 \mathrm{~h} 10$ mins

Number of sessions: minimum 6 (plus a further 4 if went forward to quit)

Pharmacotherapy: those smoking $\geq 15 \mathrm{cpd}$ used a $14 \mathrm{mg}$ nicotine patch during week 1 and increased to $21 \mathrm{mg}$ during the last 3 weeks; those smoking 10 - $14 \mathrm{cpd}$, used a $7 \mathrm{mg}$ nicotine patch during week 1 and increased to $14 \mathrm{mg}$ during the last 3 weeks; and those smoking 5 - $9 \mathrm{cpd}$ used a $7 \mathrm{mg}$ nicotine patch for the entire 4 weeks

Quit date set?: yes

Intervention 2: Nicotine gum + smoking reduction counselling as described above

Modality of support: face-to-face

Overall contact time: minimum $2 \mathrm{~h} 30$ mins, maximum $4 \mathrm{~h} 10$ mins

Number of sessions: minimum 6 (plus a further 4 if went forward to quit)

Pharmacotherapy: participants were advised to use 1 piece of $2 \mathrm{mg}$ nicotine gum for every cigarette substituted

Quit date set?: yes

\begin{tabular}{|c|c|c|}
\hline \multirow[t]{3}{*}{ Outcomes } & \multicolumn{2}{|c|}{ Definition of abstinence: 30 -day point prevalence } \\
\hline & \multicolumn{2}{|l|}{ Longest follow-up: $6 \mathrm{~m}$} \\
\hline & \multicolumn{2}{|c|}{ Biochemical validation: exhaled CO } \\
\hline Funding source & \multicolumn{2}{|c|}{ National Institute on Drug Abuse (NIDA) } \\
\hline Author conflicts of interest & \multicolumn{2}{|l|}{ Not reported } \\
\hline Notes & \multicolumn{2}{|c|}{ Relevant comparisons: 1) Reduction method versus reduction method (pharmacotherapy) } \\
\hline \multicolumn{3}{|l|}{ Risk of bias } \\
\hline Bias & Authors' judgement & Support for judgement \\
\hline $\begin{array}{l}\text { Random sequence genera- } \\
\text { tion (selection bias) }\end{array}$ & Unclear risk & Not reported \\
\hline $\begin{array}{l}\text { Allocation concealment } \\
\text { (selection bias) }\end{array}$ & Unclear risk & Not reported \\
\hline
\end{tabular}




\section{Hanson 2008 (Continued)}

Blinding of participants and personnel (performance bias)

All outcomes
High risk

Quote: "Participants were randomly assigned to receive one of the three following treatment conditions during the reduction phase: nicotine patch, nicotine gum or a placebo medication"; "Participants assigned to the placebo condition took a $400 \mathrm{mg}$ folic acid pill daily"; "there was no placebo for the nicotine patch or the nicotine gum, nor was the study double-blinded"

\begin{tabular}{lll}
\hline $\begin{array}{l}\text { Blinding of outcome as- } \\
\text { sessment (detection bias) } \\
\text { All outcomes }\end{array}$ & Low risk & Abstinence was biochemically validated \\
\hline $\begin{array}{l}\text { Incomplete outcome data } \\
\text { (attrition bias) } \\
\text { All outcomes }\end{array}$ & Unclear risk & Overall dropout was less than $50 \%$ but this is not reported split by trial arm \\
\hline Other bias & Unclear risk & $\begin{array}{l}\text { Long-term abstinence was measured but has not been reported split by study } \\
\text { group. Insufficient information to judge whether this is as a result of selective } \\
\text { reporting }\end{array}$ \\
\hline
\end{tabular}

Hao 2017

Study design: RCT
Location: China
Setting: hospital outpatient department
Recruitment: from the outpatient department of smoking cessation at Ruijin hospital

Participants $\quad \mathrm{N}=314$

Specialist population?: majority men (97\%)

Participant characteristics: 9/314 (3\%) female; average age: 51.7 y; average cig/day: 22; nicotine dependence: FTND 4.8

Preference for quitting abruptly versus gradually: not reported

Interventions

All participants received smoking cessation tips, psychological counselling, and messages via an online messaging application from doctors about smoking knowledge

Comparator: Abrupt withdrawal: participants were not asked to change their smoking consumption, but were asked to quit completely after a 3-week period

Modality of support: face-to-face, online messaging application

Overall contact time: unclear

Number of sessions: unclear

Pharmacotherapy: varenicline before and after quit day

Quit date set?: yes

Intervention: Gradual withdrawal: in the first week participants were instructed to reduce their smoking to $3 / 4$ of baseline consumption; in the 2 nd week $1 / 2$ of baseline consumption; in the third week $1 / 4$ of baseline consumption; and then to quit on day 22 .

Modality of support: face-to-face, online messaging application

Overall contact time: unclear 
Hao 2017 (Continued)

Number of sessions: unclear

Pharmacotherapy: varenicline before and after quit day

Quit date set?: yes

\begin{tabular}{ll}
\hline Outcomes & $\begin{array}{l}\text { Definition of abstinence: prolonged abstinence for } 4 \text { weeks or more } \\
\text { Longest follow-up: } 6 \mathrm{~m} \\
\text { Biochemical validation: exhaled CO }\end{array}$ \\
\hline Funding source & not reported \\
\hline Author conflicts of interest & not reported \\
\hline Notes & Relevant comparisons: 1 ) Reduction versus abrupt
\end{tabular}

\section{Risk of bias}

\begin{tabular}{|c|c|c|}
\hline Bias & Authors' judgement & Support for judgement \\
\hline \multirow[t]{2}{*}{$\begin{array}{l}\text { Random sequence genera- } \\
\text { tion (selection bias) }\end{array}$} & Unclear risk & $\begin{array}{l}\text { Quote: "All the participants were randomly assigned to the gradual withdrawal } \\
\text { and abrupt withdrawal group in the manner of } 1: 1 \text { " }\end{array}$ \\
\hline & & Comment: No further information given \\
\hline \multirow[t]{2}{*}{$\begin{array}{l}\text { Allocation concealment } \\
\text { (selection bias) }\end{array}$} & Unclear risk & $\begin{array}{l}\text { Quote: "All the participants were randomly assigned to the gradual withdrawal } \\
\text { and abrupt withdrawal group in the manner of } 1: 1 \text { " }\end{array}$ \\
\hline & & Comment: No further information given \\
\hline $\begin{array}{l}\text { Blinding of outcome as- } \\
\text { sessment (detection bias) } \\
\text { All outcomes }\end{array}$ & Low risk & Abstinence was biochemically verified \\
\hline $\begin{array}{l}\text { Incomplete outcome data } \\
\text { (attrition bias) } \\
\text { All outcomes }\end{array}$ & Low risk & $\begin{array}{l}45 / 157(28.7 \%) \text { in the gradual arm and } 58 / 157(36.9 \%) \text { in the abrupt arm were } \\
\text { lost to follow-up. Overall attrition was less than } 50 \% \text { and similar between } \\
\text { groups }\end{array}$ \\
\hline
\end{tabular}

\section{Hatsukami 2004}

\begin{tabular}{ll} 
Methods & Study design: RCT \\
& Location: USA \\
& Setting: unclear ("multicenter study at 12 sites in the United States") \\
& Recruitment: radio, newspaper, and television advertisements \\
\hline Participants & 594 \\
& Specialist population?: no \\
& Participant characteristics: $267 / 594$ (44.9\%) female; average age: 42.3 y; average cig/day: $29 ;$ nicotine \\
dependence: FTND 6.4 & Preference for quitting abruptly versus gradually: not reported
\end{tabular}


Hatsukami 2004 (Continued)

Interventions
All participants entered a 6-month treatment phase aimed at reducing the amount of smoking. Written materials suggesting smoking reduction techniques were used during brief individual counselling sessions. A target date for reducing cigarette intake by at least $50 \%$ was set within 2 weeks of enrolment. At each monthly visit participants were asked if they wanted to quit. Those who indicated a willingness to quit smoking at any time were enrolled in a 7-week cessation treatment phase followed by a 19-week cessation follow-up phase. During the cessation phase participants received abbreviated and standardised individual smoking cessation counselling through weekly visits and a treatment manual. Participants set a target quit date during the first week of cessation treatment

Comparator: Placebo: matched to bupropion treatment (described below)

Modality of support: face-to-face and telephone

Overall contact time: unclear

Number of sessions: 7 - 14 dependent on whether, and at which point, participants entered the smoking cessation treatment

Pharmacotherapy: placebo (as above)

Quit date set?: yes, if participants chose to progress to quitting treatment they set a target quit date during the first week

Intervention: Bupropion: during reduction phase bupropion for 26 weeks (150 mg for days 1 to 3 of therapy, followed by $150 \mathrm{mg}$ twice daily). During the smoking cessation treatment phase, participants received an additional 7 weeks of bupropion

Modality of support: face-to-face and telephone

Overall contact time: unclear

Number of sessions: 7 - 14 dependent on whether, and at which point, participants entered the smoking cessation treatment

Pharmacotherapy: bupropion (as above)

Quit date set?: yes; if participants chose to progress to quitting treatment they set a target quit date during the first week

Definition of abstinence: continuous

Longest follow-up: $6 \mathrm{~m}$ from beginning of cessation treatment

Biochemical validation: exhaled CO

\begin{tabular}{lll}
\hline Funding source & GlaxoSmithKline \\
\hline Author conflicts of interest & Not reported & \\
\hline Notes & Relevant comparisons: 1) Reduction method versus reduction method (pharmacotherapy) \\
\hline Risk of bias & Authors' judgement & Support for judgement \\
\hline Bias & Low risk & $\begin{array}{l}\text { Quote: "Subjects were assigned randomly using a computer-generated sched- } \\
\text { ule to either sustained-release bupropion or placebo.." }\end{array}$ \\
\hline $\begin{array}{l}\text { Random sequence genera- } \\
\text { tion (selection bias) }\end{array}$ & $\begin{array}{l}\text { Quote: "Subjects were assigned randomly using computer-generated sched- } \\
\text { ule to either sustained-release bupropion or placebo.." }\end{array}$ \\
\hline $\begin{array}{l}\text { Allocation concealment } \\
\text { (selection bias) }\end{array}$ & Unclear risk & \begin{tabular}{l} 
Comment: No further information provided \\
\hline
\end{tabular} \\
\hline
\end{tabular}


Hatsukami 2004 (Continued)

Blinding of participants Unclear risk Quote: "Current smokers were assigned randomly to receive either susand personnel (performance bias)

All outcomes tained-release bupropion (150 mg twice daily) or matching placebo"

Comment: Does not specify who was blinded

Blinding of outcome as- Low risk Abstinence was biochemically validated

sessment (detection bias)

All outcomes

Incomplete outcome data High risk

(attrition bias)

All outcomes
$179 / 295(60.7 \%)$ in the bupropion group and 169/299 (56.5\%) in the placebo group were lost to follow-up. Attrition rates were therefore high overall

\section{Haustein 2002}

\begin{tabular}{ll}
\hline Methods & Study design: RCT \\
& Location: Germany \\
& Setting: unclear \\
Recruitment: multimedia advertisements
\end{tabular}

Participants $\quad \mathrm{N}=385$

Specialist population?: no

Participant characteristics: 192/385 (49.9\%) female; average age: 41.7 y; average cig/day: 25; nicotine dependence: FTND 5.5

Preference for quitting abruptly versus gradually: not reported

Modality of support: unclear

Overall contact time: unclear

Number of sessions: unclear

Pharmacotherapy: placebo gum - 6 to 24 pieces a day for up to $9 \mathrm{~m}$

Quit date set?: yes

Comparator 2: Long-term reduction + placebo gum: participants advised to use gum whenever they felt the urge to smoke, with the goal of reducing smoking as much as possible. At 6- and 9-month follow-ups they were advised to quit smoking

Modality of support: unclear

Overall contact time: unclear

Number of sessions: unclear

Pharmacotherapy: placebo gum - 6 to 24 pieces a day for up to $9 \mathrm{~m}$

Quit date set?: no

Intervention 1: Short-term reduction + nicotine gum: participants advised to use gum whenever they felt the urge to smoke, with the goal of stopping smoking within 4 weeks, and then remaining abstinent 
Haustein 2002 (Continued)

Modality of support: unclear

Overall contact time: unclear

Number of sessions: unclear

Pharmacotherapy: $4 \mathrm{mg}$ nicotine gum - 6 to 24 pieces a day for up to $9 \mathrm{~m}$

Quit date set?: yes

Intervention 2: Long-term reduction + nicotine gum: participants advised to use gum whenever they felt the urge to smoke, with the goal of reducing smoking as much as possible. At 6 - and 9-month follow-ups they were advised to quit smoking

Modality of support: unclear

Overall contact time: unclear

Number of sessions: unclear

Pharmacotherapy: $4 \mathrm{mg}$ nicotine gum - 6 to 24 pieces a day for up to $9 \mathrm{~m}$

Quit date set?: no

\begin{tabular}{ll}
\hline Outcomes & Definition of abstinence: point prevalence \\
& $\begin{array}{l}\text { Longest follow-up: } 12 \mathrm{~m} \\
\text { Biochemical validation: exhaled CO }\end{array}$ \\
\hline Funding source & Pharmacia \\
\hline Author conflicts of interest & Not reported \\
\hline Notes & $\begin{array}{l}\text { Relevant comparisons: 1) Reduction method versus reduction method (length of reduction); 2) Reduc- } \\
\text { tion method versus reduction method (nicotine gum) }\end{array}$ \\
\hline
\end{tabular}

\section{Risk of bias}

\begin{tabular}{lll}
\hline Bias & Authors' judgement & Support for judgement \\
\hline $\begin{array}{l}\text { Random sequence genera- } \\
\text { tion (selection bias) }\end{array}$ & Low risk & $\begin{array}{l}\text { Quote: "Subjects were allocated a number and treatment code according to a } \\
\text { randomization list drawn up by Pharmacia using a computer program" }\end{array}$ \\
\hline $\begin{array}{l}\text { Allocation concealment } \\
\text { (selection bias) }\end{array}$ & Unclear risk & $\begin{array}{l}\text { Quote: "Sealed randomization envelopes were provided for each subject and } \\
\text { held by the investigator" }\end{array}$ \\
& Comment: Does not specify if these were opaque \\
\hline $\begin{array}{l}\text { Blinding of participants } \\
\text { and personnel (perfor- } \\
\text { mance bias) } \\
\text { All outcomes }\end{array}$ & Low risk & $\begin{array}{l}\text { Quote: "Placebo gum comprised chewing gum that was identical in appear- } \\
\text { ance and taste but contained no nicotine"; "The trial was conducted under } \\
\text { double-blind conditions. All study medication was identical in appearance and } \\
\text { packaging"; "Neither the investigator nor the monitor had access to the de- } \\
\text { signee's binder, in which the tags were collected, before the termination of the } \\
\text { study treatment" }\end{array}$ \\
\hline
\end{tabular}

Blinding of outcome as- Low risk Abstinence was biochemically verified sessment (detection bias)

All outcomes 
Haustein 2002 (Continued)

Incomplete outcome data High risk $\quad 50 / 96(52.1 \%)$ in short-nicotine arm; 51/97 (52.6\%) in long-nicotine arm; 59/96 (attrition bias)

$(61.5 \%)$ in short-placebo arm and 53/96 (55.2\%) in long-placebo arm were lost

All outcomes

to follow-up at $12 \mathrm{~m}$. Overall loss to follow-up was high

Ho 2018

\begin{tabular}{|c|c|}
\hline \multirow[t]{4}{*}{ Methods } & Study design: cluster-RCT \\
\hline & Location: China \\
\hline & Setting: outpatient clinic \\
\hline & $\begin{array}{l}\text { Recruitment: patients attending outpatient clinic for medical follow-ups and who met inclusion criteria } \\
\text { were invited to participate }\end{array}$ \\
\hline \multirow[t]{4}{*}{ Participants } & $N=100$ \\
\hline & Specialist population?: no \\
\hline & $\begin{array}{l}\text { Participant characteristics: 15/100 (15\%) female; average age: } 55.6 \text { y; average cig/day: 11; nicotine de- } \\
\text { pendence: } 96 \% \text { with 'mild Fagerstrom score', } 4 \% \text { with 'moderate' score }\end{array}$ \\
\hline & Preference for quitting abruptly versus gradually: not reported \\
\hline \multirow[t]{13}{*}{ Interventions } & All participants received a booklet about smoking cessation based on the AWARD model \\
\hline & $\begin{array}{l}\text { Comparator: Quit immediately: participants were warned about the health risks of smoking and ad- } \\
\text { vised to quit immediately. They were then referred to existing cessation services by providing them } \\
\text { with a hotline number and repeating this if participants failed to quit or relapsed. They also received an } \\
\text { education card that contained reduction strategies and a reduction plan }\end{array}$ \\
\hline & Modality of support: face-to-face, telephone, booklet \\
\hline & Overall contact time: 1 minute \\
\hline & Number of sessions: 5 \\
\hline & Pharmacotherapy: none \\
\hline & Quit date set?: yes \\
\hline & $\begin{array}{l}\text { Intervention: Cut down to quit: participants were advised to cut down their cigarette consumption by } \\
15 \% \text { in the first week, } 30 \% \text { in the first month, } 50 \% \text { in the third month, and eventually to quit completely } \\
\text { in the } 6 \text { th month. However, participants were allowed to have their own reduction plan as long as they } \\
\text { were committed to quit smoking within a } 6 \text {-month period. Participants were also given an education } \\
\text { card that contained reduction strategies and a suggested plan to reduce smoking }\end{array}$ \\
\hline & Modality of support: face-to-face, telephone, booklet \\
\hline & Overall contact time: unclear \\
\hline & Number of sessions: 5 \\
\hline & Pharmacotherapy: none \\
\hline & Quit date set?: yes \\
\hline
\end{tabular}

Outcomes

Definition of abstinence: 7-day point prevalence

Longest follow-up: $12 \mathrm{~m}$ 
Ho 2018 (Continued)

Biochemical validation: expired $\mathrm{CO}$ and salivary cotinine

Funding source University of Hong Kong (small project grant: 201309176051)

Author conflicts of interest None

Notes Relevant comparisons: 1) Reduction versus abrupt

Cluster-RCT as randomisation was performed based on week of recruitment, not individual participant; analysis does not account for an ICC, but the ICC is likely to be very small, and as the $95 \% \mathrm{CI}$ spans 1 any adjustment will only slightly widen the $\mathrm{Cl}$ and have no impact on conclusions

\section{Risk of bias}

\begin{tabular}{lll}
\hline Bias & Authors' judgement & Support for judgement \\
\hline $\begin{array}{l}\text { Random sequence genera- } \\
\text { tion (selection bias) }\end{array}$ & Low risk & $\begin{array}{l}\text { Randomisation was performed for weeks, not individual participants, to min- } \\
\text { imise the possibility of treatment contamination in the same clinic. The se- } \\
\text { quences were randomly computer-generated by a research assistant not } \\
\text { involved in recruitment and data collection. Although the randomisation } \\
\text { method makes it akin to a cluster RCT, review by a statistician suggests that } \\
\text { the ICC is likely to be very small and not affect study results }\end{array}$ \\
\end{tabular}

Allocation concealment High risk Quote: "To ensure allocation concealment, each generated sequence was en-

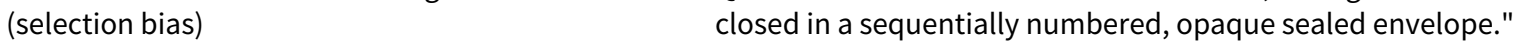

Comment: Because randomisation was performed for weeks and not participants, the researchers would have known the treatment a week was allocated to when participants attended

\begin{tabular}{lll}
\hline $\begin{array}{l}\text { Blinding of outcome as- } \\
\text { sessment (detection bias) } \\
\text { All outcomes }\end{array}$ & Low risk & Abstinence was biochemically verified \\
\hline $\begin{array}{l}\text { Incomplete outcome data } \\
\text { (attrition bias) } \\
\text { All outcomes }\end{array}$ & Low risk & $\begin{array}{l}18 / 50(36 \%) \text { in the 'Cut down to quit' group and 9/50 (18\%) in the 'Quit imme- } \\
\text { diately' group were lost to follow-up. Therefore overall loss to follow-up was } \\
\text { less than 50\% and difference in follow-up was less than 20\% }\end{array}$ \\
\hline
\end{tabular}

Hughes 2010

\begin{tabular}{ll}
\hline Methods & Study design: RCT \\
Location: USA \\
Setting: intervention remotely delivered \\
Recruitment: newspaper and radio advertisements \\
\hline
\end{tabular}

Participants $\quad \mathrm{N}=746$

Specialist population?: no

Participant characteristics: 403/746 (54\%) female; average age: 46 y; average cig/day: 23; nicotine dependence: FTND 5.9

Preference for quitting abruptly versus gradually: preference for gradual versus abrupt quitting on scale 0 - 10: 4.0, suggesting abrupt quitting slightly more popular at baseline 
Hughes 2010 (Continued)

Interventions

\section{All participants were sent the US National Cancer Institute's 'Clearing the Air' booklet}

Comparator 1: Minimal intervention: participants' plans to quit were reviewed and they were encouraged to set a quit date. Post-quit date participants were provided with relapse prevention problem-solving counselling

Modality of support: telephone

Overall contact time: $20 \mathrm{~m}$

Number of sessions: 2

Pharmacotherapy: nicotine lozenges for up to 12 weeks post-quit (those who smoked within 30 mins of rising were given $4 \mathrm{mg}$ lozenges (90\%), the rest were given $2 \mathrm{mg}$ lozenges)

Quit date set?: yes

Comparator 2: Abrupt quitting: the first call reviewed reasons for quitting, prior strategies and barriers to cessation. Participants were told not to change their smoking prior to quit day. The second call involved preparation for quitting, and the third to the fifth calls focused on relapse prevention.

Modality of support: telephone

Overall contact time: $1 \mathrm{~h} 30$ mins

Number of sessions: 5

Pharmacotherapy: nicotine lozenges for up to 12 weeks post-quit (those who smoked within 30 mins of rising were given $4 \mathrm{mg}$ lozenges (90\%), the rest were given $2 \mathrm{mg}$ lozenges)

Quit date set?: yes

Intervention: Gradual quitting: 25\% reduction in smoking recommended first week, 50\% second week, $75 \%$ third week. 4 methods of potential reduction were recommended: 1) hierarchical-easy first, 2) hierarchical-difficult first, 3) delayed reduction- delayed onset of 1st cigarette each day, 4) scheduled reduction - gradually increasing the interval between cigarettes. Recommended participants reduced over 3 weeks, but each smoker chose his/her own reduction goals and rate of progress. The first 3 calls to participants focused on reduction, 4th call discussed preparation, 5th relapse prevention

Modality of support: telephone

Overall contact time: 1 h 30 mins

Number of sessions: 5

Pharmacotherapy: nicotine lozenges for up to 12 weeks post-quit (those who smoked within 30 mins of rising were given $4 \mathrm{mg}$ lozenges (90\%), the rest were given $2 \mathrm{mg}$ lozenges)

Quit date set?: yes

Outcomes

Definition of abstinence: prolonged ( 2 weeks to $6 \mathrm{~m}$ )

Longest follow-up: $6 \mathrm{~m}$

Biochemical validation: exhaled CO

Funding source

US National Institute on Drug Abuse (grant DA-017825 to JH; Senior Scientist Award DA00490 to JH; and Institutional Training Grant DA-07242 to EP)

Author conflicts of interest

"Since 1/1/2007, Dr Hughes has received research grants from the National Institute on Health and Pfizer. Pfizer develops and sells smoking cessation medications. During this time, he has accepted honoraria or consulting fees from several non-profit and for-profit organizations and companies that develop, sell or promote smoking cessation products or services or educate/advocate about smoking cessation: Abbot Pharmaceuticals; Acrux; Aradigm; American Academy of Addiction Psychiatry; American 
Psychiatric Association; Begbies Traynor; Cambridge Hospital, Cline, Davis and Mann; Constella Group; Consultants in Behavior Change; Dean Foundation, DLA Piper, EPI-Q, European Respiratory Society, Evotec; Exchange Limited; Fagerstrom Consulting; Free and Clear; Glaxo-Smith Kline; Golin Harris; Healthwise; Insyght; Informed, Invivodata; Johns Hopkins University; JL Reckner; Maine Medical Center; McNeil Pharmaceuticals; Novartis Pharmaceuticals; Oglivy Health PR, Ottawa Heart Institute, Pfizer Pharmaceuticals; Pinney Associates; Propagate Pharmaceuticals. Reuters; Scientia, Selecta; Temple University of Health Sciences; University of Arkansas; University of California-San Francisco; University of Cantabria; University of Kentucky, US National Institutes on Health; Wolters Publishing, and Xenova. All other authors have nothing to declare"

\begin{tabular}{|c|c|c|}
\hline Notes & \multicolumn{2}{|c|}{ Relevant comparisons: 1) Reduction versus abrupt } \\
\hline \multicolumn{3}{|l|}{ Risk of bias } \\
\hline Bias & Authors' judgement & Support for judgement \\
\hline $\begin{array}{l}\text { Random sequence genera- } \\
\text { tion (selection bias) }\end{array}$ & Low risk & $\begin{array}{l}\text { A statistician generated a concealed allocation sequence and randomised par- } \\
\text { ticipants to the gradual, abrupt or brief advice conditions in a 2:2:1 ratio using } \\
\text { blocks (stratified by city and counsellor) based on the SAS, procedure PLAN }\end{array}$ \\
\hline $\begin{array}{l}\text { Allocation concealment } \\
\text { (selection bias) }\end{array}$ & Unclear risk & $\begin{array}{l}\text { Quote: "Upon receipt of consent, our statistician generated a concealed allo- } \\
\text { cation sequence" }\end{array}$ \\
\hline & & Comment: Full details of concealment methods not provided \\
\hline $\begin{array}{l}\text { Blinding of outcome as- } \\
\text { sessment (detection bias) } \\
\text { All outcomes }\end{array}$ & Low risk & Abstinence was biochemically validated \\
\hline $\begin{array}{l}\text { Incomplete outcome data } \\
\text { (attrition bias) } \\
\text { All outcomes }\end{array}$ & Low risk & $\begin{array}{l}70 / 297(23.6 \%) \text { in the gradual group, } 62 / 299(20.7 \%) \text { in the abrupt group, } \\
32 / 150(21.3 \%) \text { in the minimal intervention condition were lost to follow-up. } \\
\text { Therefore overall follow-up was less than } 50 \% \text { and similar across groups }\end{array}$ \\
\hline
\end{tabular}

\section{Risk of bias}

\begin{tabular}{|c|c|c|}
\hline Notes & \multicolumn{2}{|c|}{ Relevant comparisons: 1) Reduction versus abrupt } \\
\hline \multicolumn{3}{|l|}{ Risk of bias } \\
\hline Bias & Authors' judgement & Support for judgement \\
\hline $\begin{array}{l}\text { Random sequence genera- } \\
\text { tion (selection bias) }\end{array}$ & Low risk & $\begin{array}{l}\text { A statistician generated a concealed allocation sequence and randomised par- } \\
\text { ticipants to the gradual, abrupt or brief advice conditions in a 2:2:1 ratio using } \\
\text { blocks (stratified by city and counsellor) based on the SAS, procedure PLAN }\end{array}$ \\
\hline $\begin{array}{l}\text { Allocation concealment } \\
\text { (selection bias) }\end{array}$ & Unclear risk & $\begin{array}{l}\text { Quote: "Upon receipt of consent, our statistician generated a concealed allo- } \\
\text { cation sequence" }\end{array}$ \\
\hline & & Comment: Full details of concealment methods not provided \\
\hline $\begin{array}{l}\text { Blinding of outcome as- } \\
\text { sessment (detection bias) } \\
\text { All outcomes }\end{array}$ & Low risk & Abstinence was biochemically validated \\
\hline $\begin{array}{l}\text { Incomplete outcome data } \\
\text { (attrition bias) } \\
\text { All outcomes }\end{array}$ & Low risk & $\begin{array}{l}70 / 297(23.6 \%) \text { in the gradual group, } 62 / 299(20.7 \%) \text { in the abrupt group, } \\
32 / 150(21.3 \%) \text { in the minimal intervention condition were lost to follow-up. } \\
\text { Therefore overall follow-up was less than } 50 \% \text { and similar across groups }\end{array}$ \\
\hline
\end{tabular}

\section{Random sequence genera- Low risk} tion (selection bias)

\begin{tabular}{|c|c|c|}
\hline Notes & \multicolumn{2}{|c|}{ Relevant comparisons: 1) Reduction versus abrupt } \\
\hline \multicolumn{3}{|l|}{ Risk of bias } \\
\hline Bias & Authors' judgement & Support for judgement \\
\hline $\begin{array}{l}\text { Random sequence genera- } \\
\text { tion (selection bias) }\end{array}$ & Low risk & $\begin{array}{l}\text { A statistician generated a concealed allocation sequence and randomised par- } \\
\text { ticipants to the gradual, abrupt or brief advice conditions in a 2:2:1 ratio using } \\
\text { blocks (stratified by city and counsellor) based on the SAS, procedure PLAN }\end{array}$ \\
\hline $\begin{array}{l}\text { Allocation concealment } \\
\text { (selection bias) }\end{array}$ & Unclear risk & $\begin{array}{l}\text { Quote: "Upon receipt of consent, our statistician generated a concealed allo- } \\
\text { cation sequence" }\end{array}$ \\
\hline & & Comment: Full details of concealment methods not provided \\
\hline $\begin{array}{l}\text { Blinding of outcome as- } \\
\text { sessment (detection bias) } \\
\text { All outcomes }\end{array}$ & Low risk & Abstinence was biochemically validated \\
\hline $\begin{array}{l}\text { Incomplete outcome data } \\
\text { (attrition bias) } \\
\text { All outcomes }\end{array}$ & Low risk & $\begin{array}{l}70 / 297(23.6 \%) \text { in the gradual group, } 62 / 299(20.7 \%) \text { in the abrupt group, } \\
32 / 150(21.3 \%) \text { in the minimal intervention condition were lost to follow-up. } \\
\text { Therefore overall follow-up was less than } 50 \% \text { and similar across groups }\end{array}$ \\
\hline
\end{tabular}

Allocation concealment Unclear risk

(selection bias)

Jerome 1992

\begin{tabular}{ll}
\hline Jerome 1992 & Study design: RCT \\
\hline Lethods & Secation: USA \\
& Recruitment: advertisements in local newspapers and announcements on local radio \\
\hline Participants & Specialist population?: no \\
& $\begin{array}{l}\text { Participant characteristics: } 46 / 71 \text { (64.8\%) female; average age: } 36.8 \text { y; average cig/day: } 31.2 ; \text { nicotine } \\
\text { dependence: not reported }\end{array}$ \\
& Preference for quitting abruptly versus gradually: not reported \\
Comparator: Wait-list control (not eligible for analyses): 12 weeks after the other groups began treat- \\
ment participants were given the option of receiving the CAST + therapist assistance treatment. Partici- \\
pants who declined to participate in the treatment were not contacted further \\
Modality of support: $n / a$ \\
Onterventions \\
Overall contact time: $n / a$
\end{tabular}




\author{
Number of sessions: none \\ Pharmacotherapy: none \\ Quit date set?: no
}

Intervention 1: Smoking reduction using CAST: participants used a portable computer device and a behaviour modification-based self-help manual. In week 1 participants recorded each cigarette smoked by clicking a data input button on the device. The device then calculated and implemented an individualised rate reduction schedule by prompting users when to smoke each cigarette and gradually increasing the intervals between cigarettes until cessation was achieved. The length of the programme ranged from 17 to 35 days, depending on participants' baseline smoking rate and pattern of smoking

Modality of support: handheld computer, self-help manual

Overall contact time: $\mathrm{n} / \mathrm{a}$

Number of sessions: $\mathrm{n} / \mathrm{a}$

Pharmacotherapy: none

Quit date set?: no

Intervention 1: Smoking reduction using CAST and therapist assistance: as above, plus participants met weekly with a therapist for 40 - 70 minute counselling sessions. Support involved the presentation and discussion of various coping strategies in order to supplement the self-help instructions in the manual

Modality of support: face-to-face, handheld computer, self-help manual

Overall contact time: ranged between $1 \mathrm{~h} 20$ mins and $7 \mathrm{~h} 30$ mins

Number of sessions: 2 to 5, dependent on participant

Pharmacotherapy: none

Quit date set?: no

\title{
Outcomes
}

Definition of abstinence: 7-day point prevalence

Longest follow-up: $18 \mathrm{~m}$

Biochemical validation: exhaled CO

\begin{tabular}{ll}
\hline Funding source & Not reported \\
\hline Author conflicts of interest & $\begin{array}{l}\text { The first author was Director of Research at Health Innovations, Inc. (Reston, VA), the manufacturer of } \\
\text { the LifeSign Smoking Cessation mini-computer used in the study to reduce cigarette smoking }\end{array}$ \\
\hline Notes & $\begin{array}{l}\text { Relevant comparisons: } 1 \text { ) Reduction method versus reduction method (with versus without behaviour- } \\
\text { al support) } \\
\text { The wait-list group is not eligible for inclusion in analyses as participants were offered treatment at } 12 \\
\text { weeks and so not followed up to } 6 \mathrm{~m}\end{array}$
\end{tabular}

\section{Risk of bias}

\begin{tabular}{lll}
\hline Bias & Authors' judgement & Support for judgement \\
\hline $\begin{array}{ll}\text { Random sequence genera- } \\
\text { tion (selection bias) }\end{array}$ & Unclear risk & $\begin{array}{l}\text { Quote: "Subjects were matched on age and smoking rate, then randomly as- } \\
\text { signed to one of three groups" } \\
\end{array}$ \\
& & Comment: No further information \\
\hline
\end{tabular}


Jerome 1992 (Continued)

Allocation concealment $\quad$ Unclear risk No information provided
(selection bias)

$\begin{array}{lll}\text { Blinding of outcome as- } & \text { Low risk } & \text { Abstinence was biochemically verified } \\ \text { sessment (detection bias) } & \end{array}$

All outcomes

Incomplete outcome data Unclear risk Loss to follow-up not reported
(attrition bias)

All outcomes

Jerome 1999a

Study design: cluster-RCT
Location: USA
Setting: worksites
Recruitment: Local worksites were contacted and offered the opportunity for their employees to par-
ticipate in a free self-help smoking cessation programme. Recruiting within worksites involved various
methods such as posting advertisements and sending internal memos to employees

Participants
$\begin{aligned} & \text { Specialist population?: no } \\ & \text { Participant characteristics: } 633 / 1025(61.8 \%) \text { female; average age: } 37.5 \text { y; average cig/day: } 24 ; \text { nicotine } \\ & \text { dependence: } 632 / 1025(61.7 \%) \text { reported smoking their first cigarette of the day within } 30 \text { minutes of } \\ & \text { awakening } \\ & \text { Preference for quitting abruptly versus gradually: not reported }\end{aligned}$

Interventions

Comparator 1: General Wellness (not eligible as is a multi-behaviour intervention): printed material emphasising the importance of a general programme of physical health that included quitting smoking, exercise, and sound nutrition. Participants were advised to quit smoking but no specific techniques were recommended. Designed to be similar to what might be received from a physician or other health professional

Modality of support: self-help

Overall contact time: $\mathrm{n} / \mathrm{a}$

Number of sessions:n/a

Pharmacotherapy: none

Quit date set?: no

Comparator 2: American Lung Association self-help manual: "Freedom From Smoking For You and Your Family" (1987). This 54-page manual included standard behavioural techniques for smoking cessation based on best practice

Modality of support: self-help

Overall contact time: $\mathrm{n} / \mathrm{a}$

Number of sessions:n/a

Pharmacotherapy: none 


\section{Quit date set?: no}

Intervention: Scheduled gradual reduction: participants used the Life Sign handheld computer device (a credit card-sized computer that implemented a scheduled, gradual reduction protocol) and a 48page programme manual. Using Life Sign, participants recorded their baseline smoking over 7 days and then the computer increased their $\mathrm{ICl}$ over a period of $10-28$ days until there were no prompts to smoke. Prompts to smoke were provided by visual and auditory cues. The programme also adjusted for smokers who did not comply with the protocol by slowing the within-day increases in ICls and repeating days, thereby lengthening the programme. Some general advice on coping with urges and information on maintaining abstinence were provided by the manual

Modality of support: self-help

Overall contact time: $\mathrm{n} / \mathrm{a}$

Number of sessions:n/a

Pharmacotherapy: none

Quit date set?: no

\begin{tabular}{ll} 
Outcomes & Definition of abstinence: 7-day point prevalence \\
& $\begin{array}{l}\text { Longest follow-up: } 12 \mathrm{~m} \\
\text { Biochemical validation: exhaled CO }\end{array}$ \\
\hline Funding source & National Institute on Drug Abuse \\
\hline Author conflicts of interest & $\begin{array}{l}\text { Declaration of interest statement not provided, but the investigators were affiliated with Personal Im- } \\
\text { provement Computer Systems, Inc, a commercial interest developing computerised smoking reduction } \\
\text { products }\end{array}$
\end{tabular}

Relevant comparisons: 1 ) Reduction versus abrupt
The General Wellness group is not eligible for analyses as the intervention was multi-behaviour-fo-
cused and not comparable with the other included control interventions
This is an unpublished study supplied by the author
Cluster-RCT: analysis does not account for an ICC, but as the $95 \% \mathrm{Cl}$ spans 1 any adjustment will only
slightly widen the $\mathrm{Cl}$ and have no impact on conclusions

\section{Risk of bias}

\begin{tabular}{lll}
\hline Bias & Authors' judgement & Support for judgement \\
\hline $\begin{array}{l}\text { Random sequence genera- } \\
\text { tion (selection bias) }\end{array}$ & Unclear risk & Described as "randomized" but no further information given \\
\hline $\begin{array}{l}\text { Allocation concealment } \\
\text { (selection bias) }\end{array}$ & Unclear risk & No information provided \\
\hline $\begin{array}{l}\text { Blinding of outcome as- } \\
\text { sessment (detection bias) } \\
\text { All outcomes }\end{array}$ & Low risk & Abstinence was CO-validated \\
\hline $\begin{array}{l}\text { Incomplete outcome data } \\
\text { (attrition bias) } \\
\text { All outcomes }\end{array}$ & Low risk & $\begin{array}{l}\text { 60/415 (14.5\%) in the reduction group and 57/296 (19.3\%) in the American } \\
\text { Lung Association group were lost to follow-up at 12 m. Loss to follow-up was } \\
\text { low and similar between relevant groups }\end{array}$ \\
\hline
\end{tabular}




Study design: RCT
Location: USA
Setting: medical centre
Recruitment: newspaper, radio and poster advertising. Cardiology clinicians were invited to refer pa-
tients. A list of patients with heart disease at the medical centre, who were also documented to be
smokers, was developed from the electronic medical record and patients were invited to participate in
the study

Participants

$N=152$

Specialist population?: patients with a cardiovascular disorder

Participant characteristics: 17/152 (11.2\%) female; average age: 57.9 y; average cig/day: 27; nicotine dependence: FTND 6.0

Preference for quitting abruptly versus gradually: not reported ing for people with heart disease. Participants were encouraged to seek smoking cessation assistance from their healthcare provider(s), but no further counselling or pharmacological treatments were delivered by the study team

Modality of support: face-to-face

Overall contact time: unclear but 'brief'

Number of sessions: 1

Pharmacotherapy: none

Quit date set?: no

Intervention: Smoking reduction: the goal of treatment was to reduce smoking by at least $50 \%$ of the baseline level, or as much as possible. Participants were provided with information about the relationship between smoking and heart disease. Counsellors described specific smoking reduction strategy options, such as eliminating cigarettes at work, in the home, or least favourite or most favourite cigarettes. Participants were encouraged to choose those that were most appealing. At each visit counsellors reminded participants that abstinence from smoking was the optimal goal

Modality of support: face-to-face and telephone

Overall contact time: unclear

Number of sessions: 10 ( 5 in person, 5 by phone)

Pharmacotherapy: nicotine gum or patch: participants were encouraged to substitute a piece of $4 \mathrm{mg}$ nicotine gum for each cigarette they eliminated. If a participant was using more than 6 pieces of gum a day, or were not accomplishing reduction with gum alone, it was suggested they switch to nicotine patches

Quit date set?: no

\section{Outcomes}

Definition of abstinence: unclear

Longest follow-up: $18 \mathrm{~m}$

Biochemical validation: expired $\mathrm{CO}$ and urinary cotinine 
Joseph 2008 (Continued)

Funding source National Cancer Institute and National Institute Drug Abuse Grant (DA13333-02)

Author conflicts of interest "The authors do not have any conflicts of interest pertaining to this work"

Notes Relevant comparisons: 1 ) Reduction versus abrupt

\section{Risk of bias}

\begin{tabular}{lll}
\hline Bias & Authors' judgement & Support for judgement \\
\hline $\begin{array}{l}\text { Random sequence genera- } \\
\text { tion (selection bias) }\end{array}$ & Low risk & Quote: "by a computer-generated scheme, blocked in groups of 10 by site" \\
\hline $\begin{array}{l}\text { Allocation concealment } \\
\text { (selection bias) }\end{array}$ & Unclear risk & $\begin{array}{l}\text { Quote: "After enrollment, treatment assignment was revealed by opening a } \\
\text { sealed envelope that noted the assigned treatment condition"; }\end{array}$ \\
\hline $\begin{array}{l}\text { Blinding of outcome as- } \\
\text { sessment (detection bias) } \\
\text { All outcomes }\end{array}$ & Low risk & $\begin{array}{l}\text { Abstinence rates were measured objectively using a biochemical validation } \\
\text { method }\end{array}$ \\
\hline $\begin{array}{l}\text { Incomplete outcome data } \\
\text { (attrition bias) } \\
\text { All outcomes }\end{array}$ & Low risk & $\begin{array}{l}\text { 28/78 (35.9\%) in the reduction group and 23/51 (45.1\%) in the usual-care } \\
\text { group were lost to follow-up. Overall loss to follow-up was less than } 50 \% \text { and } \\
\text { similar between groups }\end{array}$ \\
\hline
\end{tabular}

Klemperer 2017

\begin{tabular}{|c|c|}
\hline \multirow[t]{4}{*}{ Methods } & Study design: RCT \\
\hline & Location: USA \\
\hline & Setting: remote, by telephone \\
\hline & $\begin{array}{l}\text { Recruitment: e-mail invitations sent to the Nielsen consumer panel }(\mathrm{N}=350,000) \text {. Participants in the } \\
\text { Nielsen panel are individuals who use the Internet and have elected to receive invitations to participate } \\
\text { in a variety of on-line surveys in return for points redeemable for products and services }\end{array}$ \\
\hline \multirow[t]{4}{*}{ Participants } & $N=560$ \\
\hline & Specialist population?: no \\
\hline & $\begin{array}{l}\text { Participant characteristics: 375/560 (67\%) female; average age: } 51 \text { y; average cig/day: 20; nicotine de- } \\
\text { pendence: FTND } 5.4\end{array}$ \\
\hline & Preference for quitting abruptly versus gradually: not reported \\
\hline \multirow[t]{5}{*}{ Interventions } & $\begin{array}{l}\text { All participants received an offer of a self-help booklet ( } \mathrm{NCl} \text { 's 'Clearing the Air') and a handout listing } \\
\text { medication and cessation counselling options they could pursue on their own if they attempted to quit }\end{array}$ \\
\hline & $\begin{array}{l}\text { Comparator 1: Usual care: counsellors asked questions about the participants' smoking, advised par- } \\
\text { ticipants to quit, and offered the treatment resources described above }\end{array}$ \\
\hline & Modality of support: telephone \\
\hline & Overall contact time: $5 \mathrm{~m}$ \\
\hline & Number of sessions: 1 \\
\hline
\end{tabular}


Klemperer 2017 (Continued)

Pharmacotherapy: none

Quit date set?: no

Comparator 2: Motivational intervention: based on the USPHS 5Rs protocol; the intervention included some motivational interviewing strategies. In the first call counsellors elicited and reinforced participant's reasons for wanting to quit someday, as well as their perceived risks of smoking and their perceived rewards of quitting. In the second week counsellors helped participants identify and problem-solve roadblocks to quitting. In the third call (week 4 ) counsellors reviewed and repeated messages from the first 2 calls and concluded with advice to quit smoking

Modality of support: telephone

Overall contact time: 40 mins

Number of sessions: 3

Pharmacotherapy: none

Quit date set?: no

Intervention: Reduction Intervention: the first counselling call (week 0 ) began with discussion about how reduction might increase quit attempts. Counsellors then encouraged participants to set their own goals for reduction in number of cigarettes smoked. Counsellors and participants proceeded to discuss the pros and cons of 2 strategies for reduction: (a) scheduled reduction; i.e. smoking on a schedule and increasing time between cigarettes, and (b) hierarchical reduction, eliminating certain cigarettes beginning with those that are the easiest to give up. Counsellors reviewed progress, answered questions and helped participants adjust their goals to increase chances of success during the second call (week 2). During the third call (week 4), counsellors elicited what was learned from reduction and reinforced any success that the participant reported. Counselors concluded with advice to quit smoking

Modality of support: telephone

Overall contact time: 40 mins

Number of sessions: 3

Pharmacotherapy: none

Quit date set?: no

Longest follow-up: $12 \mathrm{~m}$

Biochemical validation: none

Funding source

National Cancer Institute (grant NCI CA163176 to JRH) and National Institute on Drug Abuse (training grant T32 DA 7242-23 to EMK)

Author conflicts of interest "One of the authors received consulting and speaking fees from several companies that develop or market pharmacological and behavioral treatments for smoking cessation or harm reduction and from several non-profit organizations that promote tobacco control. He also consults (without payment) for Swedish Match."

Notes Relevant comparisons: Reduction versus abrupt

\section{Risk of bias}

Bias Authors' judgement Support for judgement


Klemperer 2017 (Continued)

Random sequence genera- Low risk tion (selection bias)
One of the investigators designed a computer-generated block randomisation schedule stratified by counsellor to assign participants to receive either intervention

\begin{tabular}{lll}
\hline $\begin{array}{l}\text { Allocation concealment } \\
\text { (selection bias) }\end{array}$ & Unclear risk & Not described
\end{tabular}

Blinding of outcome assessment (detection bias)

All outcomes
High risk

No biochemical verification of abstinence, and contact varied between some of the trial arms

Quote: "We did not use biochemical verification of abstinence, because prior telephone counseling studies found that fewer than half of participants were willing to provide samples through the mail and, more importantly, the Society for Research on Nicotine and Tobacco (SRNT) states that verification is usually not necessary when treatment contact is minimal"

Incomplete outcome data High risk
(attrition bias)

All outcomes
$102 / 186(54.8 \%)$ of the reduction group, $93 / 185$ (50.3\%) of the motivational group, and $92 / 189$ (48.7\%) of the usual-care group were lost to follow-up. Overall loss to follow-up was more than $50 \%$ at $12 \mathrm{~m}$ follow-up

Kralikova 2009

$\begin{array}{ll}\text { Methods } & \text { Study design: RCT } \\ \text { Location: Czech Republic } \\ \text { Setting: medical centres } \\ \text { Recruitment: local newspaper advertisements and local leaflets }\end{array}$

Participants $\quad \mathrm{N}=314$

Specialist population?: no

Participant characteristics: 183/314 (58\%) female; average age: 46 y; average cig/day: 25; nicotine dependence: FTND 6.0

Preference for quitting abruptly versus gradually: not reported

Comparator: Reducing to quit (as described above) + placebo

Modality of support: face-to-face

Overall contact time: 10 mins

Number of sessions: unclear

Pharmacotherapy: choice of inhaler (placebo) or gum (placebo). At $6 \mathrm{~m}$ tapering of placebo treatment began, and completely stopped at $9 \mathrm{~m}$

Quit date set?: no

Intervention: Reducing to quit (as described above) + NRT

Modality of support: face-to-face

Overall contact time: 10 mins 
Kralikova 2009 (Continued)

Number of sessions: unclear

Pharmacotherapy: choice of nicotine inhaler (10 mg) or nicotine gum (4 mg). At $6 \mathrm{~m}$ tapering of NRT treatment began, and completely stopped at $9 \mathrm{~m}$

Quit date set?: no

\begin{tabular}{ll}
\hline Outcomes & Definition of abstinence: prolonged abstinence from $6 \mathrm{~m}$ to $12 \mathrm{~m}$ \\
& Longest follow-up: $12 \mathrm{~m}$ \\
& Biochemical validation: exhaled CO \\
\hline Funding source & McNeil AB \& Farmacia CHC \\
\hline Author conflicts of interest & $\begin{array}{l}\text { "McNeil AB manufactures a range of nicotine replacement products, including nicotine gum and nico- } \\
\text { tine inhaler. Eva Kralikova and Jiri Kozakt received funding from McNeil AB to perform this study (and } \\
\text { have previously received payment from other pharmaceutical companies). Thomas Rasmussen and } \\
\text { Gunnar Gustavsson are employees of McNeil AB. Jacques Le Houezec is a consultant in tobacco depen- } \\
\text { dence for both the pharmaceutical industry and the public sector." }\end{array}$
\end{tabular}

Notes Relevant comparisons: 1) Reduction method versus reduction method (pharmacotherapy)

\section{Risk of bias}

\begin{tabular}{|c|c|c|}
\hline Bias & Authors' judgement & Support for judgement \\
\hline $\begin{array}{l}\text { Random sequence genera- } \\
\text { tion (selection bias) }\end{array}$ & Unclear risk & $\begin{array}{l}\text { Quote: "The study was placebo-controlled, randomized in a ratio of 2:1" } \\
\text { Comment: No further information provided }\end{array}$ \\
\hline $\begin{array}{l}\text { Allocation concealment } \\
\text { (selection bias) }\end{array}$ & Unclear risk & $\begin{array}{l}\text { Quote: "The study was placebo-controlled, randomized in a ratio of 2:1" } \\
\text { Comment: No further information provided }\end{array}$ \\
\hline $\begin{array}{l}\text { Blinding of participants } \\
\text { and personnel (perfor- } \\
\text { mance bias) } \\
\text { All outcomes }\end{array}$ & Unclear risk & $\begin{array}{l}\text { Quote: "a placebo-controlled double blind trial with nicotine gum and in- } \\
\text { haler"; "The placebo groups received matching treatment that did not contain } \\
\text { nicotine" } \\
\text { Comment: Does not specify who was blinded }\end{array}$ \\
\hline
\end{tabular}

Blinding of outcome as- Low risk Abstinence was biochemically verified

sessment (detection bias)

All outcomes

Incomplete outcome data Unclear risk Loss to follow-up not reported
(attrition bias)

All outcomes

\section{Lindson-Hawley 2016b}

\begin{tabular}{ll}
\hline Methods & Study design: RCT \\
& Location: UK \\
& Setting: Primary care practices
\end{tabular}


Lindson-Hawley 2016b (Continued)

Recruitment: Participants were identified through the electronic records of GP practices and invited (by letter) to take part

Participants
Specialist population?: no
Participant characteristics: $347 / 697$ (49.8\%) female; average age: 49.0 y; average cig/day: 20.0 ; nicotine
dependence: FTCD 6.0
Preference for quitting abruptly versus gradually: Abrupt = 224/697 (32.1\%); Gradual = 355/697 (50.9\%);
No preference $=118 / 697(16.9 \%)$

Interventions

All participants set a quit day 2 weeks after enrolment. Withdrawal-oriented therapy was provided weekly for 4 weeks after the quit date and at an 8-week follow-up. After the quit day, all participants were provided with $21 \mathrm{mg}$ nicotine patches and short-acting NRT of their choice and encouraged to use in response to cravings

Comparator: Abrupt cessation

Modality of support: face-to-face

Overall contact time: unclear (matched between groups)

Number of sessions: 8

Pharmacotherapy: pre-quit: $21 \mathrm{mg}$ nicotine patches. Post-quit: nicotine patches and choice of shortacting NRT

Quit date set?: yes

Intervention: Gradual cessation: participants were instructed to reduce to $50 \%$ of baseline amount by the end of the first week and $25 \%$ of baseline amount by the end of the second week in daily increments. A nurse created reduction schedules with participants. Participants could choose between hierarchical reduction, scheduled reduction or smoke-free periods reduction strategies (scheduled reduction: participants used a timer/mobile phone to schedule ICls and smoked only when the timer sounded or for 5 minutes thereafter. The time between cigarettes lengthened daily. Hierarchical reduction: participants rated cigarettes they would usually smoke from most to least favourite and progressively eliminated either their favourite or least favourite. Smoke-free periods: participants mapped their regular day and noted the 30-min periods in which they smoked. They then progressively eliminated $1 / 2$ and then $3 / 4$ of these periods).

Modality of support: face-to-face

Overall contact time: unclear (matched between groups)

Number of sessions: 8

Pharmacotherapy: pre-quit period: $21 \mathrm{mg}$ nicotine patches and choice of short-acting NRT with instructions to use 1 unit per cigarette reduced. Post-quit: nicotine patches and choice of short-acting NRT

Quit date set?: yes

Outcomes Definition of abstinence: prolonged (allowing a 2-week grace period after quit day for slips)

Longest follow-up: $6 \mathrm{~m}$

Biochemical validation: exhaled CO

Funding source British Heart Foundation

Author conflicts of interest "Dr. Lindson-Hawley reports grants from the National Institute for Health Research outside the submitted work. Dr. West reports grants, personal fees, and nonfinancial support from Pfizer and and personal fees from GlaxoSmithKline outside the submitted work. Dr. Aveyard reports grants from United King- 
Lindson-Hawley 2016b (Continued)

dom Centre for Tobacco and Alcohol Studies and the National Institute for Health Research School for Primary Care Research during the conduct of the study; and personal fees from Pfizer and McNeil outside the submitted work. Other authors disclosed no conflicts of interest."

\begin{tabular}{|c|c|c|}
\hline Notes & \multicolumn{2}{|c|}{ Relevant comparisons: 1 ) Reduction versus abrupt } \\
\hline \multicolumn{3}{|l|}{ Risk of bias } \\
\hline Bias & Authors' judgement & Support for judgement \\
\hline $\begin{array}{l}\text { Random sequence genera- } \\
\text { tion (selection bias) }\end{array}$ & Low risk & $\begin{array}{l}\text { Quote: "An independent statistician used Stata, version } 10.1 \text { (Stata-Corp), to } \\
\text { accomplish randomization stratified by research nurse, with randomly or- } \\
\text { dered blocks of } 2,4 \text {, and } 6 \text { to ensure balance." }\end{array}$ \\
\hline $\begin{array}{l}\text { Allocation concealment } \\
\text { (selection bias) }\end{array}$ & Unclear risk & $\begin{array}{l}\text { Quote: "..the research nurse opened sealed, numbered envelopes in turn." } \\
\text { Comment: Does not state whether envelopes were opaque }\end{array}$ \\
\hline $\begin{array}{l}\text { Blinding of outcome as- } \\
\text { sessment (detection bias) } \\
\text { All outcomes }\end{array}$ & Low risk & Abstinence was biochemically verified \\
\hline $\begin{array}{l}\text { Incomplete outcome data } \\
\text { (attrition bias) } \\
\text { All outcomes }\end{array}$ & Low risk & $\begin{array}{l}59 / 342(17.3 \%) \text { in the gradual cessation group and } 50 / 355(14.1 \%) \text { in the } \\
\text { abrupt cessation group were lost to follow-up at } 6 \text { months. Attrition rates were } \\
\text { low and similar between groups }\end{array}$ \\
\hline
\end{tabular}

\section{Malott 1984}

\begin{tabular}{|c|c|}
\hline \multirow[t]{4}{*}{ Methods } & Study design: RCT \\
\hline & Location: USA \\
\hline & Setting: Worksite (medical clinic or telephone company) \\
\hline & $\begin{array}{l}\text { Recruitment: "...posters and in-house newsletters announcing a smoking reduction program to be con- } \\
\text { ducted at their worksite" }\end{array}$ \\
\hline \multirow[t]{4}{*}{ Participants } & $N=24$ \\
\hline & Specialist population?: no \\
\hline & $\begin{array}{l}\text { Participant characteristics: 20/24 (83.3\%) female; average age: } 34 \text { y; average cig/day: } 24 \text {; nicotine de- } \\
\text { pendence: FTND } 6.0\end{array}$ \\
\hline & Preference for quitting abruptly versus gradually: not reported \\
\hline \multirow[t]{5}{*}{ Interventions } & $\begin{array}{l}\text { Comparator: Controlled smoking: group meetings (approximately } 50 \text { mins long) focused on sequen- } \\
\text { tially reducing nicotine content (i.e. brand of cigarette), number of cigarettes smoked per day, and per- } \\
\text { centage of each cigarette smoked. Participants attempted to achieve a } 25 \% \text { reduction in the number of } \\
\text { cigarettes smoked between sesssions } 2 \text { and } 3 \text { and an additional } 25 \% \text { reduction between sessions } 3 \text { and } \\
\text { 4. At session 4, participants were asked to set a quit date or continue reducing }\end{array}$ \\
\hline & Modality of support: group face-to-face \\
\hline & Overall contact time: $5 \mathrm{~h}$ \\
\hline & Number of sessions: 6 \\
\hline & Pharmacotherapy: none \\
\hline
\end{tabular}


Malott 1984 (Continued)

\section{Quit date set?: no}

Intervention: Controlled smoking + partner support: as above, plus participants were paired with a partner (co-workers in the same workplace) with whom he or she discussed progress on a daily basis. Each individual also received short, weekly instalments of the Partner's Controlled Smoking Manual.

Modality of support: group face-to-face

Overall contact time: $5 \mathrm{~h}$

Number of sessions: 6

Pharmacotherapy: none

Quit date set?: no

Definition of abstinence: prolonged from week 6 to final follow-up
Longest follow-up: 6 months
Biochemical validation: exhaled CO

Funding source The National Heart, Lung, and Blood Institute (\#30615)

\begin{tabular}{ll}
\hline Author conflicts of interest & Not reported \\
\hline Notes & Relevant comparisons: 1 ) Reduction method versus reduction method (with partner support)
\end{tabular}

\section{Risk of bias}

\begin{tabular}{lll}
\hline Bias & Authors' judgement & Support for judgement \\
\hline $\begin{array}{l}\text { Random sequence genera- } \\
\text { tion (selection bias) }\end{array}$ & Unclear risk & $\begin{array}{l}\text { Quote: "Participants were assigned to groups and then groups were "randomly } \\
\text { assigned to either CS or CS plus PS conditions." } \\
\text { Comment: No further information provided }\end{array}$ \\
\hline $\begin{array}{l}\text { Allocation concealment } \\
\text { (selection bias) }\end{array}$ & Unclear risk & No information provided \\
\hline $\begin{array}{l}\text { Blinding of outcome as- } \\
\text { sessment (detection bias) } \\
\text { All outcomes }\end{array}$ & Low risk & Abstinence was biochemically verified \\
\hline $\begin{array}{l}\text { Incomplete outcome data } \\
\begin{array}{l}\text { (attrition bias) } \\
\text { All outcomes }\end{array}\end{array}$ & Low risk & $\begin{array}{l}\text { o/12 (0\%) of the controlled smoking + partner support group and } 1 / 12 \text { (8.3\%) } \\
\text { of the controlled smoking group were lost to follow-up. Attrition was low and } \\
\text { similar across groups }\end{array}$ \\
\hline
\end{tabular}

\section{NCT00158158}

\begin{tabular}{ll}
\hline Methods & Study design: RCT \\
Location: USA \\
Setting: not reported \\
Recruitment: not reported \\
\hline
\end{tabular}

Participants N=200


Participant characteristics: Inclusion criteria: smoke at least 5 cigarettes a day for at least 6 months; does not regularly use other tobacco products; motivated to quit smoking; not currently using medications to quit smoking; willing to use an effective form of contraception throughout the study. Exclusion criteria: NRT is medically inadvisable; diagnosed with a psychiatric disorder within 3 months prior to enrolment; currently taking an unstable dose of psychoactive medications; currently taking medications that may react with a nicotine patch; history of alcohol or drug abuse within 3 months prior to enrolment; pregnant

Preference for quitting abruptly versus gradually: not reported

Comparator: Usual care: participants asked to set another quit day and quit abruptly

Modality of support: not reported

Overall contact time: not reported

Number of sessions: not reported

Pharmacotherapy: nicotine patches

Quit date set?: yes

Intervention: Smoking reduction: participants advised to reduce smoking rates prior to quit day. Encouraged to decrease smoking by $50 \%$ the first week and $75 \%$ the second week. During Week 3, participants will be encouraged to completely quit smoking

Modality of support: not reported

Overall contact time: not reported

Number of sessions: not reported

Pharmacotherapy: nicotine patches

Quit date set?: yes

$\begin{array}{ll}\text { Outcomes } & \text { Definition of abstinence: not reported } \\ & \text { Longest follow-up: } 6 \mathrm{~m} \\ & \text { Biochemical validation: unclear }\end{array}$

Funding source National Institute on Drug Abuse (NIDA) (NIDA-14538-2)

\section{Author conflicts of interest Not reported}

Notes Relevant comparisons: 1) Reduction versus abrupt

We classify this study as included rather than ongoing, as the trial registry lists the study as completed in 2007. However, we have been unable to identify any published data and we did not receive a response to a query to the investigator

\section{Risk of bias}

\begin{tabular}{lll}
\hline Bias & Authors' judgement & Support for judgement \\
\hline $\begin{array}{l}\text { Random sequence genera- } \\
\text { tion (selection bias) }\end{array}$ & Unclear risk & No information provided \\
\hline
\end{tabular}


NCT00158158 (Continued)

$\begin{aligned} & \text { Allocation concealment } \\ & \text { (selection bias) }\end{aligned}$
Unclear risk No information provided

\begin{tabular}{|c|c|c|}
\hline $\begin{array}{l}\text { Blinding of outcome as- } \\
\text { sessment (detection bias) } \\
\text { All outcomes }\end{array}$ & Unclear risk & No information provided \\
\hline
\end{tabular}

\begin{tabular}{lll}
\hline $\begin{array}{l}\text { Incomplete outcome data } \\
\text { (attrition bias) }\end{array}$ & Unclear risk & No information provided \\
All outcomes & Unclear risk & $\begin{array}{l}\text { The study is believed to be completed, but results have not been reported. In- } \\
\text { sufficient information to judge whether this is as a result of selective reporting }\end{array}$ \\
\hline Other bias &
\end{tabular}

\section{Nicki 1984}

\begin{tabular}{ll}
\hline Methods & Study design: RCT \\
Location: Canada \\
Setting: unclear \\
Recruitment: poster, newspaper and radio advertisements \\
\hline $\mathrm{N}=58$ \\
Specialist population?: no \\
Participant characteristics: gender not reported; average age: not reported; average cig/day: 1 pack \\
(pack size unclear); nicotine dependence: not reported \\
Preference for quitting abruptly versus gradually: not reported
\end{tabular}

Interventions

Comparator 1: Control - measures of smoking consumption taken only (not eligible for inclusion as was not randomised) by mail of "daily smoking rate and nicotine intake."

Modality of support: $n / a$

Overall contact time: $\mathrm{n} / \mathrm{a}$

Number of sessions: $\mathrm{n} / \mathrm{a}$

Pharmacotherapy: $\mathrm{n} / \mathrm{a}$

Quit date set?:n /a

Comparator 2: Nicotine-fading, self-monitoring (NF/SM): participants received information about the addictive nature of cigarette smoking. They were instructed each week to smoke cigarettes with a certain nicotine content. Over a 3-week period, in equal steps, the nicotine content was finally reduced to $0.1 \mathrm{mg} /$ cigarette or lower. Throughout this period, participants were also told to smoke as many cigarettes as they wanted. At session 5, everyone was instructed to stop smoking altogether during the following week

Modality of support: face-to-face groups

Overall contact time: $9 \mathrm{~h}$

Number of sessions: 6

Pharmacotherapy: none 
Nicki 1984 (Continued)

Quit date set?: yes

Comparator 3: NF/SM + self-talk: NF/SM as above, plus self-instructional training: participants received an explanation of how one's covert self-talk, or the omission of self-talk, may have an effect on cigarette smoking. Examples were given of appropriate self-instructions that might occur prior to, during and just after a cigarette-smoking situation. Participants were asked to develop patterns of thought, that would pertain to cigarette-smoking situations

Modality of support: face-to-face groups

Overall contact time: $9 \mathrm{~h}$

Number of sessions: 6

Pharmacotherapy: none

Quit date set?: yes

Intervention 1: NF/SM + self-efficacy: NF/SM as above, plus self-efficacy training: participants were taught about self-efficacy and its relevance to not smoking. Participants were asked to choose 1 situation where they were at high certainty of avoiding smoking and no longer to smoke in that situation. In each of the next 2 sessions, the same instruction was applied to 2 more situations of progressively lower certainty. At session 5 participants were instructed not to smoke in all situations

Modality of support: face-to-face groups

Overall contact time: $9 \mathrm{~h}$

Number of sessions: 6

Pharmacotherapy: none

Quit date set?: yes

Intervention 2: NF/SM + self-talk \& self-efficacy: a combination of all of the treatments above

Modality of support: face-to-face groups

Overall contact time: $9 \mathrm{~h}$

Number of sessions: 6

Pharmacotherapy: none

Quit date set?: yes

\begin{tabular}{ll}
\hline Outcomes & $\begin{array}{l}\text { Definition of abstinence: unclear } \\
\text { Longest follow-up: } 12 \mathrm{~m} \\
\text { Biochemical validation: none }\end{array}$ \\
\hline Funding source & Not reported \\
\hline Author conflicts of interest & Not reported \\
\hline Notes & $\begin{array}{l}\text { Relevant comparisons: } 1 \text { ) Reduction method versus reduction method (self-talk - int } 1 \text { vs. int } 2) . \text { Also in- } \\
\text { vestigates behavioural reduction as an adjunct to nicotine fading (combined } 2 \& 3 \text { vs int } 1 \& 2) \text {. This is not } \\
\text { included in the MA of reduction versus abrupt but is summarised separately }\end{array}$
\end{tabular}

\section{Risk of bias}

\section{Bias}

Authors' judgement Support for judgement 
Nicki 1984 (Continued)

Random sequence genera- Unclear risk Quote: "Apart from constraints due to Ss' availability, assignment of Ss to tion (selection bias) treatment groups before the first treatment session was random."

Cmment: No further information given

\begin{tabular}{lll}
\hline $\begin{array}{l}\text { Allocation concealment } \\
\text { (selection bias) }\end{array}$ & Unclear risk & No information given
\end{tabular}

\begin{tabular}{lll}
\hline $\begin{array}{l}\text { Blinding of outcome as- } \\
\text { sessment (detection bias) } \\
\text { All outcomes }\end{array}$ & Low risk & $\begin{array}{l}\text { Abstinence was not biochemically validated, but abstinence was verified by } \\
\text { another person and the amount of face-to-face contact between groups was } \\
\text { the same }\end{array}$ \\
\hline $\begin{array}{l}\text { Incomplete outcome data } \\
\begin{array}{l}\text { (attrition bias) } \\
\text { All outcomes }\end{array}\end{array}$ & Low risk & $\begin{array}{l}1 / 11(9.1 \%) \text { of the NF/SM group; } 1 / 13(7.7 \%) \text { of the NF/SM + ST group; } 1 / 13 \text { of } \\
\text { the NF/SM + SE group; and } 1 / 12(8.3 \%) \text { of the NF/SM + ST + SE group were lost } \\
\text { to follow-up. Attrition was low and similar across groups }\end{array}$ \\
\hline
\end{tabular}

\title{
Ostroff 2014
}

Study design: RCT
Location: USA
Setting: hospital
Recruitment: from surgical clinics based on information from patients' electronic medical records

\begin{tabular}{l} 
Participants \\
$\begin{array}{l}\text { S }=185 \\
\text { Specialist population?: "Smokers with newly diagnosed cancer" - participants were "scheduled for hos- } \\
\text { Participant characteristics: } 98 / 185 \text { (53\%) female; average age: } 55.9 \text { y; average cig/day: 20; nicotine de- } \\
\text { pendence: FTND } 4.9 \\
\text { Preference for quitting abruptly versus gradually: not reported }\end{array}$ \\
\hline
\end{tabular}

Interventions

\begin{abstract}
Comparator: Best practices only: participants were routinely advised to quit smoking by their attending surgeon during their work-up and pre-surgical consultations. All smokers were offered telephone and bedside cessation counselling on the benefits of cessation for cancer patients, potential barriers to quitting, and behavioural strategies for managing smoking urges, recommendations for use of cessation pharmacotherapy and self-help materials
\end{abstract}

Modality of support: face-to-face and telephone

Overall contact time: 1 h 20 mins - 1 h 55 mins

Number of sessions: 5

Pharmacotherapy: NRT ("pharmacotherapy recommendations were tailored to the specific needs and preferences of patients")

Quit date set?: unclear

Intervention: Best practices + scheduled reduced smoking: as above, plus a "Quitpal" - a handheld computer to administer a pre-surgical scheduled reduced smoking regimen (smokers gradually reduce their daily smoking rate by adhering to predetermined smoking times. Over days or weeks, the ICIs are gradually increased and smoking is delayed until the next scheduled cigarette). Each participant's individualised reduction schedule was tailored to 3 parameters: a) typical waking and bedtimes; b) daily average smoking rate; and c) number of days from enrolment until hospitalisation, with a quit date planned at least 24 hours prior to an inpatient admission 
Ostroff 2014 (Continued)

Modality of support: face-to-face and telephone

Overall contact time: 1 h 20 mins - 1 h 55 mins

Number of sessions: 5

Pharmacotherapy: NRT ("pharmacotherapy recommendations were tailored to the specific needs and preferences of patients"), but unclear whether it was used pre-quit as well as post-quit

Quit date set?: yes

\begin{tabular}{ll}
\hline Outcomes & Definition of abstinence: 7-day point prevalence \\
& Longest follow-up: $6 \mathrm{~m}$ \\
& Biochemical validation: salivary cotinine \\
\hline Funding source & National Cancer Institute (R01CA90514 \& T32CA009461) \\
\hline Author conflicts of interest & Not reported \\
\hline Notes & Relevant comparisons: 1 ) Reduction versus abrupt \\
\hline
\end{tabular}

\section{Risk of bias}

\begin{tabular}{lll}
\hline Bias & Authors' judgement & Support for judgement \\
\hline $\begin{array}{l}\text { Random sequence genera- } \\
\text { tion (selection bias) }\end{array}$ & Low risk & $\begin{array}{l}\text { Quote: "Computerized permuted-block randomization was conducted inde- } \\
\text { pendently by the Centers' Data Management Group." }\end{array}$ \\
\hline $\begin{array}{l}\text { Allocation concealment } \\
\text { (selection bias) }\end{array}$ & Unclear risk & No information provided \\
\hline $\begin{array}{l}\text { Blinding of outcome as- } \\
\text { sessment (detection bias) }\end{array}$ & Low risk & Abstinence was biochemically verified \\
\hline $\begin{array}{l}\text { Incomplete outcome data } \\
\text { (attrition bias) } \\
\begin{array}{l}\text { All outcomes } \\
\text { Intcomes }\end{array}\end{array}$ & Low risk & $\begin{array}{l}14 / 96 \text { (14.6\%) in the reduction group and } 14 / 89 \text { (15.7\%) in the Best practice } \\
\text { group were lost to follow-up. Loss to follow-up was low and similar across } \\
\text { groups }\end{array}$ \\
\hline
\end{tabular}

Perez-Milena 2012

\begin{tabular}{ll}
\hline Methods & Study design: RCT \\
Location: Spain \\
Setting: high schools \\
Recruitment: through school counsellors by simple random sampling, stratified by high school \\
\hline $\mathrm{N}=91$ \\
Specialist population?: teenage high school students \\
Participant characteristics: $45 / 91$ (49.5\%) female; average age: 15.4 y; average cig/day: $12 ;$ nicotine de- \\
pendence: FTND 3.0 \\
Preference for quitting abruptly versus gradually not reported
\end{tabular}


Perez-Milena 2012 (Continued)

Interventions All participants were sent reminders by SMS on their quit day, the day before and the week after, as well as monthly emails for a year

Comparator: Brief intervention: participants were given abrupt smoking cessation advice and set a quit day

Modality of support: face-to-face and text message

Overall contact time: 15 mins

Number of sessions: 1

Pharmacotherapy: none

Quit date set?: yes

Intervention: Intensive intervention: in weeks 1 and 2 participants were advised to progressively reduce their smoking consumption by $30 \%$ of baseline consumption; they were advised to quit in week 3

Modality of support: face-to-face and text message

Overall contact time: $1 \mathrm{~h}$

Number of sessions: 4

Pharmacotherapy: none

Quit date set?: yes

\begin{tabular}{ll} 
Outcomes & Definition of abstinence: continuous \\
& $\begin{array}{l}\text { Longest follow-up: } 12 \mathrm{~m} \\
\text { Biochemical validation: exhaled CO }\end{array}$ \\
\hline Funding source & Biomedical and Health Sciences Research in Andalusia (PI 0160/2008) \\
\hline Author conflicts of interest & The authors report no conflicts \\
\hline Notes & Relevant comparisons: 1 ) Reduction versus abrupt \\
\hline
\end{tabular}

\section{Risk of bias}

\begin{tabular}{lll}
\hline Bias & Authors' judgement & Support for judgement \\
\hline $\begin{array}{l}\text { Random sequence genera- } \\
\text { tion (selection bias) }\end{array}$ & Low risk & Randomisation was carried out using the computer programme Epidat 3.1 \\
\hline $\begin{array}{l}\text { Allocation concealment } \\
\text { (selection bias) }\end{array}$ & Unclear risk & $\begin{array}{l}\text { Blind allocation to each group is mentioned, but it is unclear how this was } \\
\text { achieved }\end{array}$ \\
\hline $\begin{array}{l}\text { Blinding of outcome as- } \\
\text { sessment (detection bias) } \\
\text { All outcomes }\end{array}$ & Low risk & Abstinence was biochemically verified \\
\hline $\begin{array}{l}\text { Incomplete outcome data } \\
\text { (attrition bias) } \\
\text { All outcomes }\end{array}$ & Low risk & $\begin{array}{l}1 / 43(2.3 \%) \text { of the intensive group and 2/48 (4.2\%) of the brief group were lost } \\
\text { to follow-up; attrition was low and similar across groups }\end{array}$ \\
\hline
\end{tabular}


Rennard 2006

\begin{tabular}{|c|c|}
\hline \multirow[t]{4}{*}{ Methods } & Study design: RCT \\
\hline & Location: USA \\
\hline & Setting: unclear \\
\hline & Recruitment: newspaper advertisements \\
\hline \multirow[t]{4}{*}{ Participants } & $N=429$ \\
\hline & Specialist population?: no \\
\hline & $\begin{array}{l}\text { Participant characteristics: } 237 / 429 \text { (55.3\%) female; average age: } 45.3 \text { y; average cig/day: } 30 ; \text { nicotine } \\
\text { dependence: FTND } 6.6\end{array}$ \\
\hline & Preference for quitting abruptly versus gradually: not reported \\
\hline \multirow[t]{13}{*}{ Interventions } & $\begin{array}{l}\text { All participants were instructed to reduce their smoking as much as possible and were provided with } \\
\text { information on possible ways to do so (no further detail given). Smoking cessation was recommended } \\
\text { from month } 6 \text { as the long-term goal }\end{array}$ \\
\hline & Comparator: Placebo inhaler \\
\hline & Modality of support: face-to-face \\
\hline & Overall contact time: unclear \\
\hline & Number of sessions: unclear \\
\hline & $\begin{array}{l}\text { Pharmacotherapy: placebo inhaler with } 1 \mathrm{mg} \text { of menthol. Inhalers could be used ad libitum, with a rec- } \\
\text { ommended dose of } 6 \text { to } 12 \text { cartridges a day, for up to } 12 \text { months }\end{array}$ \\
\hline & Quit date set?: no \\
\hline & Intervention: Nicotine inhaler \\
\hline & Modality of support: face-to-face \\
\hline & Overall contact time: unclear \\
\hline & Number of sessions: unclear \\
\hline & $\begin{array}{l}\text { Pharmacotherapy: } 10 \mathrm{mg} \text { nicotine inhaler with } 1 \mathrm{mg} \text { of menthol. Inhalers could be used ad libitum, with } \\
\text { a recommended dose of } 6 \text { to } 12 \text { cartridges a day, for up to } 12 \text { months }\end{array}$ \\
\hline & Quit date set?: no \\
\hline
\end{tabular}

Outcomes Definition of abstinence: 7-day point prevalence

Longest follow-up: $15 \mathrm{~m}$

Biochemical validation: exhaled $\mathrm{CO}$

\begin{tabular}{ll}
\hline Funding source & Not reported \\
\hline Author conflicts of interest & $\begin{array}{l}\text { 4 of the authors were affiliated with Pfizer Consumer Healthcare, Helsinborg, Sweden, the manufactur- } \\
\text { er of the experimental treatment }\end{array}$ \\
\hline Notes & Relevant comparisons: 1) Reduction method versus reduction method (pharmacotherapy)
\end{tabular}

\section{Risk of bias}

Bias Authors' judgement Support for judgement


Rennard 2006 (Continued)

Random sequence genera- Unclear risk $\quad$ No information provided
tion (selection bias)

\begin{tabular}{lll}
\hline $\begin{array}{l}\text { Allocation concealment } \\
\text { (selection bias) }\end{array}$ & Unclear risk & No information provided
\end{tabular}

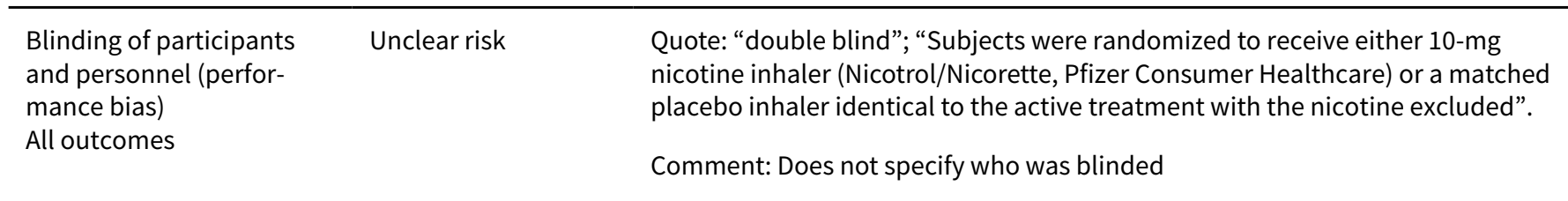

\begin{tabular}{lll}
\hline $\begin{array}{l}\text { Blinding of outcome as- } \\
\text { sessment (detection bias) } \\
\text { All outcomes }\end{array}$ & Low risk & Abstinence was biochemically verified \\
\hline $\begin{array}{l}\text { Incomplete outcome data } \\
\text { (attrition bias) }\end{array}$ & High risk & $\begin{array}{l}126 / 215(59 \%) \text { of the nicotine inhaler group and 149/214 (70\%) of the placebo } \\
\text { inhaler group were lost to follow-up. Overall attrition was high }\end{array}$ \\
\hline
\end{tabular}

Riley 2001

\begin{tabular}{|c|c|}
\hline \multirow[t]{4}{*}{ Methods } & Study design: RCT \\
\hline & Location: USA \\
\hline & Setting: unclear \\
\hline & Recruitment: television media advertisements \\
\hline \multirow[t]{4}{*}{ Participants } & $N=337$ \\
\hline & Specialist population?: no \\
\hline & $\begin{array}{l}\text { Participant characteristics: 148/337 (44\%) female; average age: } 41 \text { y; average cig/day: 24; nicotine de- } \\
\text { pendence: not reported }\end{array}$ \\
\hline & Preference for quitting abruptly versus gradually: not reported \\
\hline \multirow[t]{9}{*}{ Interventions } & $\begin{array}{l}\text { Comparator: Nicotine patch only: participants were advised to stop smoking abruptly and then begin } \\
\text { using nicotine patch }\end{array}$ \\
\hline & Modality of support: unclear \\
\hline & Overall contact time: unclear \\
\hline & Number of sessions: unclear \\
\hline & Pharmacotherapy: nicotine patches after the quit day \\
\hline & Quit date set?: unclear \\
\hline & $\begin{array}{l}\text { Intervention: Computerised scheduled gradual reduction + patch: a handheld computer was used to } \\
\text { schedule the reduction of smoking rate by increasing the interval between smoking of cigarettes over } \\
10-21 \text { days depending on initial smoking rate. When smoking rate was down to } 10 \mathrm{cpd} \text { participants } \\
\text { were advised to stop smoking completely and start the use of nicotine patches }\end{array}$ \\
\hline & Modality of support: unclear \\
\hline & Overall contact time: unclear \\
\hline
\end{tabular}


Riley 2001 (Continued)

Number of sessions: unclear

Pharmacotherapy: nicotine patches after the quit day

Quit date set?: no (as reduction was tailored to individual progress)

Definition of abstinence: unclear
Longest follow-up: $12 \mathrm{~m}$, but results only reported to 12 week follow-up so far
Biochemical validation: unclear

Funding source National Cancer Institute: R44CA71305

Author conflicts of interest Declaration of interest statement not provided, but the investigators were affiliated with Personal Improvement Computer Systems, Inc, a commercial interest developing computerised smoking reduction products

Relevant comparisons: 1 ) Reduction versus abrupt
Long-term follow-up data have not been published. We have had contact with authors who still have
the data but have not analysed it. Would be willing to do so, but were not able to supply results in time
for the completion of this review

\section{Risk of bias}

\begin{tabular}{lll}
\hline Bias & Authors' judgement & Support for judgement \\
\hline $\begin{array}{l}\text { Random sequence genera- } \\
\text { tion (selection bias) }\end{array}$ & Unclear risk & Not reported \\
\hline $\begin{array}{l}\text { Allocation concealment } \\
\text { (selection bias) }\end{array}$ & Unclear risk & Not reported \\
\hline $\begin{array}{l}\text { Blinding of outcome as- } \\
\text { sessment (detection bias) } \\
\text { All outcomes }\end{array}$ & Unclear risk & Not reported \\
\hline $\begin{array}{l}\text { Incomplete outcome data } \\
\text { (attrition bias) } \\
\text { All outcomes }\end{array}$ & Unclear risk & Not reported \\
\hline $\begin{array}{l}\text { Other bias } \\
\end{array}$ & Unclear risk & $\begin{array}{l}\text { Long-term abstinence was measured but has not been reported. Insufficient } \\
\text { information to judge whether this is as a result of selective reporting }\end{array}$ \\
\hline
\end{tabular}

\section{Riley 2005}

\begin{tabular}{ll}
\hline Methods & Study design: RCT \\
& Location: USA \\
& Setting: minimal contact (remote) \\
& Recruitment: participants responded to local television spots \\
\hline Participants & $\mathrm{N}=423$ \\
& Specialist population?: no
\end{tabular}


Riley 2005 (Continued)

Participant characteristics: 186/423 (44\%) female; average age: 43.4 y; average cig/day: not reported; nicotine dependence: NDSS 0.06 in LifeSign group; 0.25 in nasal spray only group

Preference for quitting abruptly versus gradually: not reported

Interventions
Comparator: Nasal spray only: participants were instructed to select a quit day sometime in the next week, quit smoking, and begin using the nasal spray as indicated in the package insert

Modality of support: brief face-to-face

Overall contact time: 10 mins

Number of sessions: 1

Pharmacotherapy: nicotine nasal spray after quit day: participants received 2 units and a brief orientation on use. They were instructed to call to receive refills during the 10 -week trial

Quit date set?: yes

Intervention: LifeSign + nasal spray: participants received a LifeSign research unit programmed with the LS-NS programme. The programme was used to record smoking for a 7-day baseline period; the computer then began to prompt the decreasing use of cigarettes and increasing use of nasal spray over 10 days. When time to smoke the computer flashed a smoke symbol and produced a low sustained beep, and when time for nasal spray flashed a nasal spray symbol and produced a higher, shorter beep. Participants recorded cigarettes smoked and nasal spray usage using assigned buttons. Participants were then expected to quit smoking and use nasal spray only. The computer prompted each spray use and participants recorded each spray use, and each cigarette smoked (if slips occurred). After 3 weeks of stable nasal spray dosing the programme gradually weaned participants off nasal spray

Modality of support: brief face-to-face and LifeSign handheld device

Overall contact time: 10 mins

Number of sessions: 1

Pharmacotherapy: nicotine nasal spray before and after quit day: participants received 2 units and a brief orientation on use. They were instructed to call to receive refills during the 10 -week trial. The LifeSign device signalled when participants should use the nasal spray

Quit date set?: yes

Longest follow-up: $12 \mathrm{~m}$

Biochemical validation: cotinine

\begin{tabular}{ll}
\hline Funding source & Not reported \\
\hline Author conflicts of interest & $\begin{array}{l}\text { Declaration of interest statement not provided, but the investigators were affiliated with Personal Im- } \\
\text { provement Computer Systems, Inc, a commercial interest developing computerised smoking reduction } \\
\text { products }\end{array}$
\end{tabular}

Notes Relevant comparisons: 1) Reduction versus abrupt

\section{Risk of bias}

\begin{tabular}{lll}
\hline Bias & Authors' judgement & Support for judgement \\
\hline $\begin{array}{l}\text { Random sequence genera- } \\
\text { tion (selection bias) }\end{array}$ & Unclear risk & No information provided \\
\hline
\end{tabular}


Riley 2005 (Continued)

$\begin{aligned} & \text { Allocation concealment } \\ & \text { (selection bias) }\end{aligned}$
Unclear risk No information provided

\begin{tabular}{|c|c|c|}
\hline $\begin{array}{l}\text { Blinding of outcome as- } \\
\text { sessment (detection bias) } \\
\text { All outcomes }\end{array}$ & Low risk & Abstinence was biochemically validated \\
\hline
\end{tabular}

\begin{tabular}{|c|c|c|}
\hline $\begin{array}{l}\text { Incomplete outcome data } \\
\text { (attrition bias) } \\
\text { All outcomes }\end{array}$ & High risk & $\begin{array}{l}\text { Overall } 57 \% \text { of participants were lost to follow-up (attrition was not reported } \\
\text { split by arm) }\end{array}$ \\
\hline
\end{tabular}

\title{
Rohsenow 2016
}

Study design: RCT
Location: USA
Setting: state-funded residential substance use disorder (SUD) treatment programmes
Recruitment: from patients admitted to the SUD treatment programme after any detoxification was
complete

Participants N=340

Specialist population?: People with a substance use disorder

Participant characteristics: 112/340 (33\%) female; average age: 37.6 y; average cig/day: 20; nicotine dependence: FTND 5.9

Preference for quitting abruptly versus gradually: not reported

Interventions

\begin{abstract}
All participants received brief advice over 4 sessions; therapist advised smoking cessation for health reasons, gave advice about useful methods, and asked participants to set a quit date within the next week. A handout on common barriers to smoking cessation, a consumer guide for smoking cessation, published pamphlets on smoking cessation and hard candy were provided on request. In sessions 2 - 4 participants were reminded of health reasons for quitting, engaged in problem-solving around barriers, noted successes and methods they should continue using and reminded them of methods. Although the nature of these methods is unclear the following excerpt suggests they were reduction methods: "Reduction phase. Breath CO level was collected late each afternoon for 5 days. Participants received a printed voucher with a monetary value of $\$ 2$ per test for a $25 \%$ reduction from baseline co level, $\$ 4$ for $50 \%$ reduction, and $\$ 6$ for a $75 \%$ or greater reduction". This reduction phase was preceded by a baseline phase and followed by an abstinence phase.
\end{abstract}

\begin{abstract}
Comparator: Contingent vouchers: during a 5-day reduction phase breath CO level was collected late each afternoon for 5 days. Participants received a printed voucher with a monetary value of USD 2 per test for a $25 \%$ reduction from baseline CO level, USD 4 for $50 \%$ reduction, and USD 6 for a $75 \%$ or greater reduction. In the following 14-day abstinence phase breath CO level was collected twice a day. An escalating schedule of payments provided increasing levels of payments in vouchers for each successive CO reading $\leq 6 \mathrm{ppm}$, starting at USD 3 for the first sample, and increasing by USD 0.50 for each consecutive negative test to USD 16.50 for the 28th consecutive abstinent breath sample, plus USD 10 bonuses provided every time 3 consecutive readings showed abstinence. When a breath sample did not indicate abstinence the participant earned no voucher and the payment schedule reverted to the initial USD 3 level, then after 3 consecutive abstinent samples the schedule returned to the payment level at which the reset occurred. Participants who completed all 19 days of samples and missed no more than 3 of the scheduled breath tests earned a USD 40 bonus voucher (total possible = USD $433+$ USD 33 for showing up = USD 466)
\end{abstract}

Modality of support: face-to-face 
Overall contact time: $1 \mathrm{~h}$

Number of sessions: 4

Pharmacotherapy: nicotine patches for 8 weeks from start of study $(21 \mathrm{mg} /$ day for 4 weeks, $14 \mathrm{mg} /$ day for 2 weeks, and $7 \mathrm{mg} /$ day for 2 weeks)

Quit date set?: yes

Intervention: Non-contingent vouchers - payments were received for providing breath samples over 19 days non-contingent on reduction or abstinence, plus the added $\$ 33$ the contingent group received simply for providing samples as scheduled, and a $\$ 40$ bonus for providing all 33 samples (total possible $=\$ 304)$.

Modality of support: face to face

Overall contact time: $1 \mathrm{~h}$

Number of sessions: 4

Pharmacotherapy: nicotine patches for 8 weeks from start of study $(21 \mathrm{mg} /$ day for 4 weeks, $14 \mathrm{mg} /$ day for 2 weeks, and $7 \mathrm{mg} /$ day for 2 weeks)

Quit date set?: yes

\begin{tabular}{ll}
\hline Outcomes & Definition of abstinence: 7-day point prevalence \\
& Longest follow-up: $12 \mathrm{~m}$ \\
& Biochemical validation: exhaled CO and salivary cotinine
\end{tabular}

Funding source

National Institute on Drug Abuse (1 R01 DA023995); Department of Veterans Affairs (Senior Career Research Scientist Award to Rohsenow); National Institute on Alcohol Abuse and Alcoholism (K05AA019681)

\begin{tabular}{|c|c|c|}
\hline Author conflicts of interest & \multicolumn{2}{|l|}{ Not reported } \\
\hline Notes & \multicolumn{2}{|c|}{$\begin{array}{l}\text { Relevant comparisons: } 1 \text { ) Reduction method versus reduction method (contingent vs. non-contingent } \\
\text { rewards) }\end{array}$} \\
\hline \multicolumn{3}{|l|}{ Risk of bias } \\
\hline Bias & Authors' judgement & Support for judgement \\
\hline $\begin{array}{l}\text { Random sequence genera- } \\
\text { tion (selection bias) }\end{array}$ & Low risk & $\begin{array}{l}\text { Quote: "Urn randomization [...] on the first day of the voucher period stratified } \\
\text { by gender, Fagerström Test for Nicotine Dependence, and Smoking Contem- } \\
\text { plation Ladder scores." }\end{array}$ \\
\hline $\begin{array}{l}\text { Allocation concealment } \\
\text { (selection bias) }\end{array}$ & Unclear risk & No information provided \\
\hline $\begin{array}{l}\text { Blinding of outcome as- } \\
\text { sessment (detection bias) } \\
\text { All outcomes }\end{array}$ & Low risk & Abstinence was biochemically validated \\
\hline $\begin{array}{l}\text { Incomplete outcome data } \\
\text { (attrition bias) } \\
\text { All outcomes }\end{array}$ & Low risk & $\begin{array}{l}46 / 172(26.7 \%) \text { in the contingent group and } 54 / 168(32.1 \%) \text { in the non-contin- } \\
\text { gent group were lost to follow-up at } 12 \mathrm{~m} \text { follow-up. Attrition was under } 50 \% \\
\text { and similar between groups }\end{array}$ \\
\hline
\end{tabular}


Ruther 2018

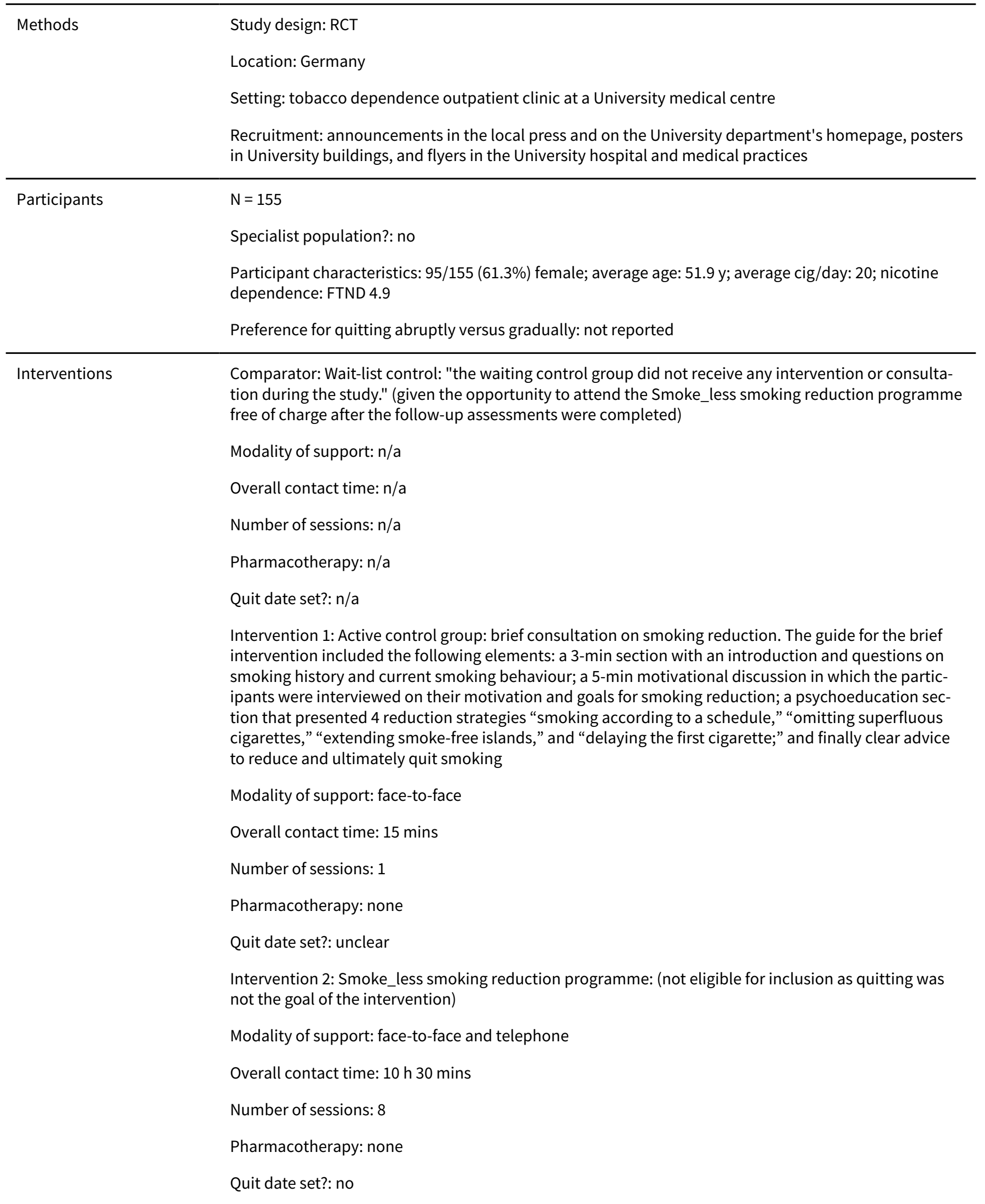


Ruther 2018 (Continued)

Longest follow-up: $6 \mathrm{~m}$

Biochemical validation: no

\begin{tabular}{ll}
\hline Funding source & "The authors have no support or funding to report" \\
\hline Author conflicts of interest & $\begin{array}{l}\text { "T Rüther has been a consultant for, received grant/research support and honoraria from and been a } \\
\text { speaker for or on the advisory board of Johnson \& Johnson, and Pfizer. O. Pogarell has been on the } \\
\text { advisory board of Lundbeck and received speaker's honoraria from Lundbeck, Desitin, and Otsuka. A. } \\
\text { Kiss, K. Eberhardt, A. Linhardt and C. Kröger declare no conflicts of interest" }\end{array}$ \\
\hline Notes & Relevant comparisons: 1) Reduction versus no treatment \\
\hline
\end{tabular}

\section{Risk of bias}

\begin{tabular}{|c|c|c|}
\hline Bias & Authors' judgement & Support for judgement \\
\hline $\begin{array}{l}\text { Random sequence genera- } \\
\text { tion (selection bias) }\end{array}$ & Low risk & $\begin{array}{l}\text { Quote: "A computer-generated list of random numbers was used to randomly } \\
\text { assign participants in a 1:1:1 ratio" }\end{array}$ \\
\hline $\begin{array}{l}\text { Allocation concealment } \\
\text { (selection bias) }\end{array}$ & Unclear risk & No information provided \\
\hline $\begin{array}{l}\text { Blinding of outcome as- } \\
\text { sessment (detection bias) } \\
\text { All outcomes }\end{array}$ & High risk & $\begin{array}{l}\text { Wait-list control was used, meaning that people in this arm may have been less } \\
\text { likely to try and quit and may have been waiting to receive treatment to do so. } \\
\text { Abstinence was not biochemically validated }\end{array}$ \\
\hline $\begin{array}{l}\text { Incomplete outcome data } \\
\text { (attrition bias) } \\
\text { All outcomes }\end{array}$ & Low risk & $\begin{array}{l}2 / 49(4.1 \%) \text { in the active control group and } 7 / 55(12.7 \%) \text { in the wait-list control } \\
\text { were lost to follow-up at } 6 \mathrm{~m} \text {. Attrition was low and similar between groups }\end{array}$ \\
\hline
\end{tabular}

\section{Shiffman 2009}

\begin{tabular}{ll}
\hline Methods & Study design: RCT \\
Location: USA \\
Setting: simulated over-the-counter (OTC) setting \\
Recruitment: print and radio advertisements
\end{tabular}
Participants
$N=3297$
Specialist population?: no
Participant characteristics: 1879/3297 (57\%) female; average age: 44.2 y; average cig/day: 25; nicotine dependence: FTND 5.7
Preference for quitting abruptly versus gradually: not reported tained from a printed user's guide and label. No instruction, counselling, or intervention was provided by study personnel. The materials instructed participants to use the gum to reduce cigarettes per day until they achieved $24 \mathrm{~h}$ of abstinence and then use the gum to maintain abstinence
Comparator: Placebo gum
Modality of support: printed self-help materials 
Shiffman 2009 (Continued)

Overall contact time: $\mathrm{n} / \mathrm{a}$

Number of sessions: $\mathrm{n} / \mathrm{a}$

Pharmacotherapy: participants self-selected their study gum 'dosage' (2 or $4 \mathrm{mg}$ ) after reviewing the labels for both doses, which told smokers of 25 cigarettes a day to select the $4 \mathrm{mg}$ dose

Quit date set?: no

Intervention: Nicotine gum

Modality of support: printed self-help materials

Overall contact time: $\mathrm{n} / \mathrm{a}$

Number of sessions: $\mathrm{n} / \mathrm{a}$

Pharmacotherapy: participants self-selected their study gum dosage ( 2 or $4 \mathrm{mg}$ ) after reviewing the labels for both doses, which told smokers of 25 cigarettes per day to select the $4 \mathrm{mg}$ dose

Quit date set?: no

\begin{tabular}{ll}
\hline Outcomes & Definition of abstinence: continuous \\
& Longest follow-up: $6 \mathrm{~m}$ \\
& Biochemical validation: exhaled CO \\
\hline Funding source & SmithKline Beecham Consumer Healthcare (now GlaxoSmithKline Consumer Healthcare) \\
\hline Author conflicts of interest & $\begin{array}{l}\text { "Dr. Strahs is employed by GSKCH. Through their work at Pinney Associates, Drs. Shiffman and Fergu- } \\
\text { son serve as consultants to GSKCH on matters related to smoking control and/or nicotine replacement } \\
\text { medications. Dr. Shiffman also has a financial interest in a venture to develop new nicotine replace- } \\
\text { ment medications. The authors had full access to all of the data in the study and take responsibility for } \\
\text { the integrity of the data and the accuracy of the data analysis." }\end{array}$
\end{tabular}

\section{Risk of bias}

\begin{tabular}{|c|c|c|}
\hline Bias & Authors' judgement & Support for judgement \\
\hline $\begin{array}{l}\text { Random sequence genera- } \\
\text { tion (selection bias) }\end{array}$ & Low risk & $\begin{array}{l}\text { Quote: "Using a 1:1 computer-generated randomization scheme, balanced } \\
\text { across study sites and generated separately for the } 2 \text {-and } 4 \text {-mg groups, partic- } \\
\text { ipants were randomized on a doubleblind basis to receive active or placebo } \\
\text { gum" }\end{array}$ \\
\hline $\begin{array}{l}\text { Allocation concealment } \\
\text { (selection bias) }\end{array}$ & Unclear risk & $\begin{array}{l}\text { Specifies that participants were randomised on a double-blind basis, but does } \\
\text { not specify methods }\end{array}$ \\
\hline $\begin{array}{l}\text { Blinding of participants } \\
\text { and personnel (perfor- }\end{array}$ & Unclear risk & $\begin{array}{l}\text { Quote: "This was a multi-center, placebo-controlled, double-blind RCT of 2- } \\
\text { and 4-mg nicotine gum versus placebo". }\end{array}$ \\
\hline All outcomes & & $\begin{array}{l}\text { Comment: No description of placebo and does not explicitly state who was } \\
\text { blinded }\end{array}$ \\
\hline $\begin{array}{l}\text { Blinding of outcome as- } \\
\text { sessment (detection bias) } \\
\text { All outcomes }\end{array}$ & Low risk & Abstinence was biochemically verified \\
\hline
\end{tabular}


Shiffman 2009 (Continued)

Incomplete outcome data (attrition bias)

All outcomes
Unclear risk

The numbers lost to follow-up is unclear, as authors excluded anyone who failed to quit from follow-ups. It is impossible to separate these numbers from those who were lost for other reasons

\section{Wang 2017}

Study design: cluster-RCT
Location: China
Setting: unclear
Recruitment: trained smoking cessation ambassadors actively recruited adult smokers from the com-
munity

Participants N N 1077

Specialist population?: no

Participant characteristics: 201/1077 (18.6\%) female; average age: 42.7 y; average cig/day: 15; nicotine dependence: N and \% Heaviness of Smoking Index (HSI) Nicotine dependence > 4: 382/1077 (35.5\%)

Preference for quitting abruptly versus gradually: not reported

Interventions

All participants received a standard 12-page smoking cessation booklet, and booster messages (1 - 2 mins) by telephone at 1 week, 1 month, and 2 months

Comparator: Quit immediately (QI): participants received brief QI advice and a QI card, and brief smoking cessation advice over the telephone at follow-up. the The brief advice was AWARD advice (about 5 mins) focused on quitting immediately and ambassadors encouraged participants to set a quit day close to baseline.

Modality of support: face-to-face and telephone

Overall contact time: $10 \mathrm{~m}$

Number of sessions: 4

Pharmacotherapy: none

Quit date set?: yes

Intervention: Cut down to quit: participants received brief advice and a card on smoking reduction at baseline; thereafter, on follow-up, they received brief smoking reduction advice over the telephone. Brief advice (5 mins) on smoking reduction used the 'Ask, Warn, Advise, Refer, Do it again' model, which included advising participants to quit by cutting down cigarette consumption at their own pace within 3 months. Smoking cessation ambassadors helped participants to set strategies for gradual reduction, such as reduction in the number of cigarettes smoked per day by $25 \%$ in the first week, $50 \%$ in the first month, $75 \%$ in the second month and quit altogether in the third month, OR scheduled reduction by increasing time intervals between each cigarette, OR the hierarchical reduction approach, starting with the easiest cigarette of the day to forgo and moving to the hardest cigarette to give up (or vice versa). The card also contained the information above

Modality of support: face-to-face and telephone

Overall contact time: $10 \mathrm{~m}$

Number of sessions: 4

Pharmacotherapy: none 
Wang 2017 (Continued)

$$
\text { Quit date set?: yes }
$$

\begin{tabular}{ll} 
Outcomes & Definition of abstinence: 7 -day point prevalence \\
& Longest follow-up: $6 \mathrm{~m}$ \\
& Biochemical validation: salivary cotinine \\
\hline Funding source & Council on Smoking and Health (COSH) \\
\hline Author conflicts of interest & "None declared" \\
\hline Notes & Relevant comparisons: 1 ) Reduction versus abrupt \\
& $\begin{array}{l}\text { Cluster-RCT: an ICC of } 0.01 \text { was used to calculate the study sample size, which is very low and likely to } \\
\text { have minimal impact. The analysis does not account for an ICC, but as the } 95 \% \text { CI spans } 1 \text { any adjust- } \\
\text { ment will only slightly widen the Cl and have no impact on conclusions }\end{array}$
\end{tabular}

\section{Risk of bias}

\section{Bias}

\section{Authors' judgement Support for judgement}

Random sequence genera- Unclear risk tion (selection bias)

Quote: "...using permuted block randomization. The primary investigator, who was not involved in the recruitment, randomly generated blocks, with each block size being equal to 4 and containing a random permutation of the 2 groups. All the blocks were combined to generate the list of group allocation."

Comment: The CDTQ group had a significantly higher proportion of participants in paid employment, higher daily cigarette consumption and higher nicotine dependence level (HSI score $\geq 4$ )

\begin{tabular}{|c|c|c|}
\hline $\begin{array}{l}\text { Allocation concealment } \\
\text { (selection bias) }\end{array}$ & Unclear risk & $\begin{array}{l}\text { Quote: "The recruitment staff was informed about the group allocation one } \\
\text { day prior to the recruitment session. The subjects were not informed about the } \\
\text { intervention in other groups." }\end{array}$ \\
\hline
\end{tabular}

Blinding of outcome as- Low risk Abstinence was biochemically validated

All outcomes

\begin{tabular}{ll}
$\begin{array}{l}\text { Incomplete outcome data } \\
\text { (attrition bias) }\end{array}$ & Low risk \\
All outcomes & $\begin{array}{l}\text { 202/559 (36.1\%) of the 'Cut down to quit' group and 214/518 (41.3\%) of the } \\
\text { 'Quit immediately' group were lost to follow-up. Attrition was less than } 50 \% \\
\text { overall and similar between groups }\end{array}$ \\
\hline
\end{tabular}

Wennike 2003

\begin{tabular}{ll}
\hline Methods & Study design: RCT \\
& Location: Denmark \\
& Setting: clinic \\
& Recruitment: newspaper advertisements \\
\hline Participants & S $=411$ \\
& Specialist population?: no \\
& Participant characteristics: $254 / 411(61.8 \%)$ female; average age: 44.5 y; average cig/day: $24 ;$ nicotine \\
dependence: FTND 6.4
\end{tabular}


Wennike 2003 (Continued)

Preference for quitting abruptly versus gradually: not reported

Interventions

All participants received information on behavioural smoking reduction and the general implications of smoking and its effects on health parameters. They were asked to reduce their daily number of cigarettes as much as possible by increasing the intervals between cigarettes, or increasing the time to first cigarette in the morning, or removing habitual cigarettes. Smoking cessation was recommended as the ultimate goal throughout the study

Comparator: placebo gum

Modality of support: face-to-face

Overall contact time: ranged between $2 \mathrm{~h} 15$ mins and $4 \mathrm{~h} 30$ mins

Number of sessions: 9

Pharmacotherapy: placebo gum was provided free of charge for ad libitum use for up to 12 months

Quit date set?: no

Intervention: nicotine gum

Modality of support: face-to-face

Overall contact time: ranged between $2 \mathrm{~h} 15$ mins and $4 \mathrm{~h} 30$ mins

Number of sessions: 9

Pharmacotherapy: participants who scored $\leq 5$ in the Fagerström test were allocated to $2 \mathrm{mg}$ nicotine gum and those scoring 6 - 10 were allocated to $4 \mathrm{mg}$ nicotine gum. Nicotine gum was provided free of charge for ad libitum use for up to $12 \mathrm{~m}$

Quit date set?: no

\begin{tabular}{ll}
\hline Outcomes & Definition of abstinence: 7-day point prevalence \\
& Longest follow-up: $24 \mathrm{~m}$ \\
& Biochemical validation: exhaled CO \\
\hline Funding source & Pharmacia AB, Sweden \\
\hline Author conflicts of interest & Not reported \\
\hline Notes & Relevant comparisons: 1 ) Reduction method versus reduction method (pharmacotherapy) \\
\hline
\end{tabular}

\section{Risk of bias}

\begin{tabular}{lll}
\hline Bias & Authors' judgement & Support for judgement \\
\hline $\begin{array}{l}\text { Random sequence genera- } \\
\text { tion (selection bias) }\end{array}$ & Unclear risk & No information given \\
\hline $\begin{array}{l}\text { Allocation concealment } \\
\text { (selection bias) }\end{array}$ & Unclear risk & No information given \\
\hline $\begin{array}{l}\text { Blinding of participants } \\
\text { and personnel (perfor- } \\
\text { mance bias) } \\
\text { All outcomes }\end{array}$ & Unclear risk & $\begin{array}{l}\text { Quote: "This 2-year, double-blind, randomized, placebo controlled trial with } \\
\text { parallel groups tested the efficacy and safety of nicotine gum in smoking re- } \\
\text { duction"; "The placebo gum was similar in appearance and taste, but con- } \\
\text { tained no nicotine". }\end{array}$ \\
\hline
\end{tabular}


Wennike 2003 (Continued)

Blinding of outcome as- Low risk Abstinence was biochemically verified sessment (detection bias)

All outcomes

$\begin{array}{ll}\begin{array}{l}\text { Incomplete outcome data } \\ \text { (attrition bias) }\end{array} & \text { High risk } \\ \text { All outcomes } & \text { Quote: "153 (37\%) completed the 24-month study" } \\ & \begin{array}{l}\text { Comment: Therefore overall attrition was high, and was not reported split by } \\ \text { groups }\end{array}\end{array}$

\section{Wu 2017}

Study design: RCT
Location: China
Setting: "Endocrinology and Acupuncture out-patient clinics"
Recruitment: all patients attending the Endocrinology and Acupuncture out-patient clinics were invited
to take part

Participants N=369

Specialist population?: male hospital outpatients; $71 \%$ had the following diseases: heart disease, lung disease, diabetes, hypertension, cancer, other

Participant characteristics: 0/369 (0\%) female; average age: 40.4 y; average cig/day: 10 - 19: 160/369 (43\%); $\geq 20: 209 / 369$ (57\%); nicotine dependence: FTND: 174/369 (47.2\%) scored 0 - 3; 49/369 (13.3) scored 4 - 5, and 146/369 (39.6\%) scored $6-10$

Preference for quitting abruptly versus gradually: not reported pants received brief face-to-face advice ( $1 \mathrm{~min}$ ) about exercise and diet, and then follow-up counselling over the phone

Modality of support: face-to-face and telephone

Overall contact time: $6 \mathrm{~m}$

Number of sessions: 6

Pharmacotherapy: none

Quit date set?: no

Intervention: Smoking reduction intervention: brief face-to-face advice (1 min) about smoking. Participants were then told to reduce their cigarette consumption by $\geq 50 \%$ within 1 month. It was explained that reducing smoking should be an intermediate step before complete cessation. Participants received follow-up counselling over the phone

Modality of support: face-to-face and telephone

Overall contact time: $6 \mathrm{~m}$

Number of sessions: 6

Pharmacotherapy: none

Quit date set?: no 
Wu 2017 (Continued)

Longest follow-up: $12 \mathrm{~m}$

Biochemical validation: exhaled CO

Funding source

National Natural Science Foundation of China (81373080), the Beijing Municipal Science and Technology Commission (Z121107001012070) and the Chinese PLA General Hospital (2013FC-TSYS-1021 and MJ201447)

\begin{tabular}{|c|c|c|}
\hline Author conflicts of interest & "None" & \\
\hline Notes & \multicolumn{2}{|c|}{ Relevant comparisons: 1) Reduction versus no treatment } \\
\hline \multicolumn{3}{|l|}{ Risk of bias } \\
\hline Bias & Authors' judgement & Support for judgement \\
\hline $\begin{array}{l}\text { Random sequence genera- } \\
\text { tion (selection bias) }\end{array}$ & Low risk & $\begin{array}{l}\text { Quote: "A research assistant of the project generated the random numbers for } \\
\text { group assignment using a computer" }\end{array}$ \\
\hline $\begin{array}{l}\text { Allocation concealment } \\
\text { (selection bias) }\end{array}$ & Low risk & $\begin{array}{l}\text { Quote: "After written consent, a trained counsellor who was not involved in } \\
\text { preparing the randomization sequence opened a serially numbered, opaque } \\
\text { and sealed envelope with a card inside indicating intervention or control and } \\
\text { randomly allocated the participant accordingly, thus ensuring allocation con- } \\
\text { cealment" }\end{array}$ \\
\hline $\begin{array}{l}\text { Blinding of outcome as- } \\
\text { sessment (detection bias) } \\
\text { All outcomes }\end{array}$ & Low risk & Abstinence was biochemically verified \\
\hline $\begin{array}{l}\text { Incomplete outcome data } \\
\text { (attrition bias) } \\
\text { All outcomes }\end{array}$ & Low risk & $\begin{array}{l}60 / 181(33.2 \%) \text { in the smoking reduction group and } 59 / 188(31.4 \%) \text { in the exer- } \\
\text { cise and diet advice group were lost to follow-up. Attrition was under } 50 \% \text { and } \\
\text { similar across groups }\end{array}$ \\
\hline
\end{tabular}

CBT: cognitive behavioural therapy; CO: carbon monoxide; cpd, cigs/day: cigarettes per day; FTND: Fagerström Test for Nicotine Dependence; h: hour; ICC: interclass correlation coefficient; ICI: inter-cigarette interval; m: month; min: minute; n/a: not applicable; NRT: nicotine replacement therapy; ppm: parts per million; RCT: randomised controlled trial; y: year

Characteristics of excluded studies [ordered by study ID]

\begin{tabular}{ll}
\hline Study & Reason for exclusion \\
\hline ACTRN12609000482268 & No behavioural smoking reduction intervention \\
\hline ACTRN12617000905369 & Follow-up less than 6 months following baseline \\
\hline Applegate 2004 & Aim was not to quit \\
\hline Armitage 2007 & No behavioural smoking reduction intervention \\
\hline Atwood 1975 & Follow-up less than 6 months following baseline \\
\hline Audrain McGovern 2011 & Aim was not to quit \\
\hline Aveyard 2011 & Not a randomised study \\
\hline Aveyard 2014 & Not a randomised study \\
\hline F
\end{tabular}




\begin{tabular}{|c|c|}
\hline Study & Reason for exclusion \\
\hline Baker 2006 & No behavioural smoking reduction intervention \\
\hline Barbarin 1978 & No suitable control \\
\hline Batra 2003 & Aim was not to quit \\
\hline Batra 2005 & Aim was not to quit \\
\hline Beavers 1973 & Follow-up less than 6 months following baseline \\
\hline Becona 1991 & Not a randomised study \\
\hline Becona 1993 & No suitable control \\
\hline Becona 1998 & Intervention only included nicotine and/or tar fading, not reduction in smoking behaviour \\
\hline Becona 2001 & Intervention only included nicotine and/or tar fading, not reduction in smoking behaviour \\
\hline Becona Iglesias 1989 & Not a randomised study \\
\hline Berecz 1971 & No behavioural smoking reduction intervention \\
\hline Berecz 1984 & Did not measure cessation \\
\hline Berger 2006 & Not a randomised study \\
\hline Bernard 1972 & Follow-up less than 6 months following baseline \\
\hline Blalock 2001 & Follow-up less than 6 months following baseline \\
\hline Bloch 2010 & No behavioural smoking reduction intervention \\
\hline Bolliger 2000b & Aim was not to quit \\
\hline Borland 1999 & Aim was not to quit \\
\hline Borland 2013 & No behavioural smoking reduction intervention \\
\hline Bowers 1987 & Intervention only included nicotine and/or tar fading, not reduction in smoking behaviour \\
\hline Bradford 1991 & Not a randomised study \\
\hline Brown 1984 & Intervention only included nicotine and/or tar fading, not reduction in smoking behaviour \\
\hline Brue 2001 & Follow-up less than 6 months following baseline \\
\hline Buceta 1989 & Not a randomised study \\
\hline Burling 1982 & Not a randomised study \\
\hline Burling 1989 & Intervention only included nicotine and/or tar fading, not reduction in smoking behaviour \\
\hline Burling 1994 & Intervention only included nicotine and/or tar fading, not reduction in smoking behaviour \\
\hline Burris 2014 & Aim was not to quit \\
\hline
\end{tabular}




\begin{tabular}{|c|c|}
\hline Study & Reason for exclusion \\
\hline Cacciapaglia 2007 & Follow-up less than 6 months following baseline \\
\hline Caponnetto 2013 & Aim was not to quit \\
\hline Caponnetto 2014 & Aim was not to quit \\
\hline Cassidy 2018 & Follow-up less than 6 months following baseline \\
\hline Chambliss 1979 & Not a randomised study \\
\hline Chen 2013 & Follow-up less than 6 months following baseline \\
\hline Cinciripini 1994 & Not a randomised study \\
\hline Colby 2005 & No behavioural smoking reduction intervention \\
\hline Colletti 1978 & No behavioural smoking reduction intervention \\
\hline Colletti 1979 & No behavioural smoking reduction intervention \\
\hline Colletti 1980 & No behavioural smoking reduction intervention \\
\hline Colletti 1982 & Not a randomised study \\
\hline Corty 1984 & No behavioural smoking reduction intervention \\
\hline Cropsey 2008 & No behavioural smoking reduction intervention \\
\hline Cropsey 2015 & Not a randomised study \\
\hline Crosbie 1972 & No behavioural smoking reduction intervention \\
\hline D'Ruiz 2017 & Follow-up less than 6 months following baseline \\
\hline Darity 1997 & No behavioural smoking reduction intervention \\
\hline Daughton 1994 & Follow-up less than 6 months following baseline \\
\hline Delahunt 1977 & No behavioural smoking reduction intervention \\
\hline Dlack 1999 & Aim was not to quit \\
\hline Dogris 1998 & Follow-up less than 6 months following baseline \\
\hline Ebbert 2010 & Aim was not to quit \\
\hline Ehrsam 1991 & No behavioural smoking reduction intervention \\
\hline Elliott 1978 & No behavioural smoking reduction intervention \\
\hline Emmons 1988 & Intervention only included nicotine and/or tar fading, not reduction in smoking behaviour \\
\hline Etringer 1984 & Intervention only included nicotine and/or tar fading, not reduction in smoking behaviour \\
\hline Etter 2011 & Follow-up less than 6 months following baseline \\
\hline
\end{tabular}




\begin{tabular}{|c|c|}
\hline Study & Reason for exclusion \\
\hline Euler 1973 & Follow-up less than 6 months following baseline \\
\hline Evins 2001 & No behavioural smoking reduction intervention \\
\hline Evins 2007 & No behavioural smoking reduction intervention \\
\hline Fagerström 2002a & Not a randomised study \\
\hline Farkas 2001 & Follow-up less than 6 months following baseline \\
\hline Fatemi 2005 & No behavioural smoking reduction intervention \\
\hline Fatemi 2013 & No behavioural smoking reduction intervention \\
\hline Feryo 2009 & Not a randomised study \\
\hline Filia 2010 & Intervention targeted multiple lifestyle factors, not just smoking \\
\hline Forgays 1987 & No behavioural smoking reduction intervention \\
\hline Foxx 1979 & Intervention only included nicotine and/or tar fading, not reduction in smoking behaviour \\
\hline Foxx 1983 & Not a randomised study \\
\hline Franklin 2009 & Aim was not to quit \\
\hline Frederiksen 1976 & Not a randomised study \\
\hline Fuhrer 1972 & Follow-up less than 6 months following baseline \\
\hline Galizia 1990 & Not a randomised study \\
\hline Gariti 2002 & No behavioural smoking reduction intervention \\
\hline Gelkopf 2012 & Aim was not to quit \\
\hline Gil Roales-Nieto 1992b & Aim was not to quit \\
\hline Glasgow 1983 & Aim was not to quit \\
\hline Glasgow 1984 & Aim was not to quit \\
\hline Glasgow 1986 & Aim was not to quit \\
\hline Glasgow 2009b & Follow-up less than 6 months following baseline \\
\hline Gonzalez 1991 & Intervention only included nicotine and/or tar fading, not reduction in smoking behaviour \\
\hline Gradl 2009 & Not a randomised study \\
\hline Graff 1966 & Follow-up less than 6 months following baseline \\
\hline Gulliver 2008 & No behavioural smoking reduction intervention \\
\hline Gutmann 1967 & Aim was not to quit \\
\hline
\end{tabular}




\begin{tabular}{|c|c|}
\hline Study & Reason for exclusion \\
\hline Gylys 2000 & Follow-up less than 6 months following baseline \\
\hline Hamilton 1998 & Intervention only included nicotine and/or tar fading, not reduction in smoking behaviour \\
\hline Hatsukami 1988 & Aim was not to quit \\
\hline Hatsukami 2005 & Aim was not to quit \\
\hline Hawk 2015 & Follow-up less than 6 months following baseline \\
\hline Hilleman 1994 & No behavioural smoking reduction intervention \\
\hline Hills 1982 & Follow-up less than 6 months following baseline \\
\hline Hovell 2009 & No behavioural smoking reduction intervention \\
\hline Hughes 1991 & No behavioural smoking reduction intervention \\
\hline Hughes 2004b & No behavioural smoking reduction intervention \\
\hline Hughes 2007 & Not a randomised study \\
\hline Hughes 2011 & Aim was not to quit \\
\hline Hughes 2016 & Not a randomised study \\
\hline Hurt 1990 & Not a randomised study \\
\hline ISRCTN13288677 & Aim was not to quit \\
\hline ISRCTN13837944 & Follow-up less than six months following baseline \\
\hline ISRCTN64013828 & Follow-up less than 6 months following baseline \\
\hline Jacobs 1971 & No behavioural smoking reduction intervention \\
\hline Jeon 2016 & No behavioural smoking reduction intervention \\
\hline Jerome $1999 b$ & No behavioural smoking reduction intervention \\
\hline Joksic 2011 & No behavioural smoking reduction intervention \\
\hline Joseph 2005 & Aim was not to quit \\
\hline Karam-Hage 2014 & No behavioural smoking reduction intervention \\
\hline Karoly 1975 & No behavioural smoking reduction intervention \\
\hline КСТ0001277 & No behavioural smoking reduction intervention \\
\hline Kelly 2010 & Follow-up less than 6 months following baseline \\
\hline Keutzer 1968 & No behavioural smoking reduction intervention \\
\hline Klein 2010 & No behavioural smoking reduction intervention \\
\hline
\end{tabular}




\begin{tabular}{|c|c|}
\hline Study & Reason for exclusion \\
\hline Klemperer 2016 & Not a randomised study \\
\hline Lamb 2004 & No behavioural smoking reduction intervention \\
\hline Lamb 2007 & Follow-up less than 6 months following baseline \\
\hline Lamb 2010 & Follow-up less than 6 months following baseline \\
\hline Lan 2007 & Follow-up less than 6 months following baseline \\
\hline Lando 1985 & Intervention only included nicotine and/or tar fading, not reduction in smoking behaviour \\
\hline Larson 1999 & Follow-up less than 6 months following baseline \\
\hline Leischow 2004 & Not a randomised study \\
\hline Levinson 1971 & Follow-up less than 6 months following baseline \\
\hline Levinson 2008 & Aim was not to quit \\
\hline Lichtenstein 1967 & No behavioural smoking reduction intervention \\
\hline Lillington 1998 & Not a randomised study \\
\hline Macgregor 1996 & Not a randomised study \\
\hline Marston 1971 & Did not measure cessation \\
\hline Meredith 2011 & Not a randomised study \\
\hline Mihaltan 2007 & Not a randomised study \\
\hline Morris 2011 & No behavioural smoking reduction intervention \\
\hline Mueller 2012 & Aim was not to quit \\
\hline Muramoto 1999 & No behavioural smoking reduction intervention \\
\hline Murray 1981 & Aim was not to quit \\
\hline Myette 1993 & Not a randomised study \\
\hline Myles 1994 & Not a randomised study \\
\hline Møller 2002 & Did not measure cessation \\
\hline NCT01772641 & Study terminated due to difficulty recruiting and retaining participants \\
\hline NCT01982110 & No behavioural smoking reduction intervention \\
\hline NCT03128554 & Did not measure cessation \\
\hline Nentwig 1978 & Aim was not to quit \\
\hline Newman 1982 & Aim was not to quit \\
\hline
\end{tabular}




\begin{tabular}{|c|c|}
\hline Study & Reason for exclusion \\
\hline Noonan 2018 & Follow-up less than 6 months following baseline \\
\hline NTR5113 & No behavioural smoking reduction intervention \\
\hline O'Brien 2015 & No behavioural smoking reduction intervention \\
\hline O'Connor 1998 & Not a randomised study \\
\hline Olbrich 2008 & Follow-up less than 6 months following baseline \\
\hline Orleans 1991 & Intervention only included nicotine and/or tar fading, not reduction in smoking behaviour \\
\hline Patten 1996 & Follow-up less than 6 months following baseline \\
\hline Pisinger 2005 & Not a randomised study \\
\hline Relinger 1977 & Follow-up less than 6 months following baseline \\
\hline Rennard 1993 & Aim was not to quit \\
\hline Rennard 2010 & No behavioural smoking reduction intervention \\
\hline Riggs 2001 & Follow-up less than 6 months following baseline \\
\hline Riley 2002 & Aim was not to quit \\
\hline Ritchie 1991 & Follow-up less than 6 months following baseline \\
\hline Robey 2015 & Not a randomised study \\
\hline Rose 2010 & No behavioural smoking reduction intervention \\
\hline Rovina 2003 & No behavioural smoking reduction intervention \\
\hline Royce 1995 & No behavioural smoking reduction intervention \\
\hline Rutter 1990 & Aim was not to quit \\
\hline Salk 1976 & Not a randomised study \\
\hline Schiller 2012 & Participants were smokeless tobacco users \\
\hline Schinke 1978 & Aim was not to quit \\
\hline Schleicher 2010 & No behavioural smoking reduction intervention \\
\hline Schuurmans 2016 & Not a randomised study \\
\hline Schwartz 1967 & Follow-up less than 6 months following baseline \\
\hline Scott 1986 & Not a randomised study \\
\hline Severson 2000 & Participants were smokeless tobacco users \\
\hline Sipich 1974 & No behavioural smoking reduction intervention \\
\hline
\end{tabular}




\begin{tabular}{|c|c|}
\hline Study & Reason for exclusion \\
\hline Smith 2017 & Not a randomised study \\
\hline Spanos 1993 & No behavioural smoking reduction intervention \\
\hline Srivastava 2007 & Not a randomised study \\
\hline Stein 2002 & Aim was not to quit \\
\hline Steinberg 2018 & Did not measure cessation \\
\hline Stitzer 1985 & Follow-up less than 6 months following baseline \\
\hline Suedfeld 1974 & No behavioural smoking reduction intervention \\
\hline Sutherland 1975 & No behavioural smoking reduction intervention \\
\hline Templer 1969 & No behavioural smoking reduction intervention \\
\hline Thompson 2016 & Follow-up less than 6 months following baseline \\
\hline Thorndike 2006 & Follow-up less than 6 months following baseline \\
\hline Thuerauf 2007 & Follow-up less than 6 months following baseline \\
\hline Tidey 2002 & Follow-up less than 6 months following baseline \\
\hline Tseng 2016 & Aim was not to quit \\
\hline Tønnesen 2005 & Aim was not to quit \\
\hline Weidberg 2018 & Follow-up less than 6 months following baseline \\
\hline Wetter 2006 & Not a randomised study \\
\hline Wewers 2009 & No behavioural smoking reduction intervention \\
\hline White 2011 & No behavioural smoking reduction intervention \\
\hline Wiseman 1998 & Not a randomised study \\
\hline
\end{tabular}

Characteristics of studies awaiting assessment [ordered by study ID]

Cinciripini 2001

\begin{tabular}{ll}
\hline Methods & Unknown \\
\hline Participants & Unknown \\
\hline Interventions & Unknown \\
\hline Outcomes & Unknown \\
\hline Funding source & Unknown \\
\hline
\end{tabular}


Cinciripini 2001 (Continued)

Author conflicts of interest Unknown

Notes Relevant comparisons: unknown

This is a conference abstract that we have been unable to locate, titled: 'Scheduled smoking and transdermal nicotine replacement'. We have therefore been unable to carry out a full eligibility assessment. We have contacted the lead author without response.

\section{Cooper 1990}

\begin{tabular}{ll}
\hline Methods & Unknown \\
\hline Participants & Unknown \\
\hline Interventions & Unknown \\
\hline Outcomes & Unknown \\
\hline Funding source & Unknown \\
\hline Author conflicts of interest & Unknown \\
\hline
\end{tabular}

Notes Relevant comparisons: unknown

This is a conference abstract that we have been unable to locate, titled: 'Behaviour modification and nicotine reduction therapy with heavy smokers: Comparison of four different dosing strategies'. We have therefore been unable to carry out a full eligibility assessment. We contacted the lead author, but they were no longer able to locate the associated conference presentation information.

Engeln 1969

\begin{tabular}{ll}
\hline Methods & Unknown \\
\hline Participants & Unknown \\
\hline Interventions & Unknown \\
\hline Outcomes & Unknown \\
\hline Funding source & Unknown \\
\hline Author conflicts of interest & Unknown \\
\hline Notes & $\begin{array}{l}\text { Relevant comparisons: unknown } \\
\text { This is a dissertation that we have been unable to locate, titled: 'A comparison of desensitization } \\
\text { and aversive conditioning as treatment methods to reduce cigarette smoking'. We have therefore } \\
\text { been unable to carry out a full eligibility assessment. We did not contact the author as we were un- } \\
\text { able to locate their contact details, and due to the age of the dissertation we would be unlikely to } \\
\text { locate additional information. }\end{array}$ \\
\hline
\end{tabular}


Gardner 1971

\begin{tabular}{ll}
\hline Methods & Unknown \\
\hline Participants & Unknown \\
\hline Interventions & Unknown \\
\hline Outcomes & Unknown \\
\hline Funding source & Unknown \\
\hline Author conflicts of interest & Unknown \\
\hline Notes & $\begin{array}{l}\text { Relevant comparisons: unknown } \\
\text { This is a dissertation that we have been unable to locate, titled: 'A test of coverant control therapy } \\
\text { to reduce cigarette smoking: A comparative study of the effectiveness of two different strategies } \\
\text { with a direct test of the effectiveness of contingency management'. We have therefore been unable } \\
\text { to carry out a full eligibility assessment. We did not contact the author as we were unable to locate } \\
\text { their contact details, and due to the age of the dissertation we would be unlikely to locate addition- } \\
\text { al information. }\end{array}$ \\
\hline
\end{tabular}

Palmer 1983

\begin{tabular}{ll}
\hline Methods & Unknown \\
\hline Participants & Unknown \\
\hline Interventions & Unknown \\
\hline Outcomes & Unknown \\
\hline Funding source & Unknown \\
\hline Author conflicts of interest & Unknown \\
\hline Notes & Relevant comparisons: unknown \\
& $\begin{array}{l}\text { This is a dissertation that we have been unable to locate, titled: 'A multiple stage treatment for } \\
\text { smoking reduction'. We have therefore been unable to carry out a full eligibility assessment. We did } \\
\text { not contact the author as we were unable to locate their contact details, and due to the age of the } \\
\text { dissertation we would be unlikely to locate additional information. }\end{array}$ \\
\hline
\end{tabular}

\section{Rennard 1994}

\begin{tabular}{ll}
\hline Methods & Unknown \\
\hline Participants & Unknown \\
\hline Interventions & Unknown \\
\hline Outcomes & Unknown \\
\hline
\end{tabular}


Rennard 1994 (Continued)

\begin{tabular}{ll} 
Funding source & Unknown \\
\hline Author conflicts of interest & Unknown \\
\hline Notes & Relevant comparisons: unknown \\
& $\begin{array}{l}\text { This is a conference abstract that we have been unable to locate, titled: 'The effects of nicotine re- } \\
\text { placement therapy on cigarette smoking reduction'. We have therefore been unable to carry out a } \\
\text { full eligibility assessment. We have contacted the lead author without response. }\end{array}$
\end{tabular}

Weis 1974

\begin{tabular}{ll}
\hline Methods & Unknown \\
\hline Participants & Unknown \\
\hline Interventions & Unknown \\
\hline Outcomes & Unknown \\
\hline Funding source & Unknown \\
\hline Author conflicts of interest & Unknown \\
\hline Notes & Relevant comparisons: unknown \\
& $\begin{array}{l}\text { This is a dissertation that we have been unable to locate, titled: 'An experimental examination of } \\
\text { Cautela's covert sensitization as a smoking reduction technique'. We have therefore been unable } \\
\text { to carry out a full eligibility assessment. We did not contact the author as we were unable to locate } \\
\text { their contact details, and due to the age of the dissertation we would be unlikely to locate addition- } \\
\text { al information. }\end{array}$ \\
\hline
\end{tabular}

Characteristics of ongoing studies [ordered by study ID]

\section{Hagens 2017}

\begin{tabular}{ll}
\hline Trial name or title & The REDUQ trial \\
\hline Methods & Study design: RCT \\
& Location: Netherlands \\
& Setting: outpatient hospital pulmonary clinics \\
& Recruitment: from medical records, by chest physicians during outpatient hospital visits, through \\
& leaflets and posters in waiting rooms, and through advertisements in local newspapers
\end{tabular}

\section{Participants}

\section{Goal N $=262$}

Specialist population?: people aged 40 - 80 years with COPD

Eligibility criteria: a clinical diagnosis of COPD, aged 40 - 80 years, smoking $\geq 10$ cigarettes per day, no intention to quit within the next month (i.e. not ready to quit) but interested in reducing smoking, had made 2 or more failed lifetime quit attempts. Patients pregnant or intending to become 
Hagens 2017 (Continued)

pregnant within the next 18 months, who have a serious psychological condition, who are contraindicated for NRT, or have insufficient comprehension of the Dutch language, are excluded

Interventions

Comparator: Self-help reduction: one-off meeting addressing themes like smoking in relation to COPD, self-monitoring, high-risk situations, ways to reduce the number of cigarettes smoked, and the use of NRT to aid smoking reduction. A non-tailored self-help manual was provided, describing the scheduled reduced smoking procedure and other ways to reduce smoking, as well as tips on how to cope with urges to smoke and stress, and how to prevent relapse.

Intervention: Intensive reduction: Group sessions comprising education, group discussion, sharing of experiences, and strategies to improve participants' self-efficacy to achieve and sustain reduced smoking levels. Participants received a comprehensive workbook, containing written information on all aspects of the intervention, and homework assignments to be carried out prior to each session. Telephone sessions were tailored to the individual's needs and progress towards the reduction objective and addressed current smoking status and experiences with smoking reduction and NRT. To reduce smoking, 'scheduled reduced smoking' was used: smokers were instructed to smoke only at prespecified times of the day and the interval between cigarettes was progressively increased. Individualised smoking reduction schedules were constructed according to baseline smoking rate, daily wakening cycle and success at meeting intermediate reduction goals. The first treatment week, participants were expected to follow a schedule without reducing consumption. Participants were subsequently instructed to reduce by $25 \%$ between weeks 2 and 4 and by $50 \%$ in weeks 4 to week 8 . At each meeting, participants who were unsuccessful in meeting their reduction goal were motivated to reach this goal by the next meeting. From week 8 to week 13 , those successful in achieving a 50\% or greater reduction were highly encouraged to consider cessation and enter cessation treatment. If they were not ready to quit smoking completely they were given the options to reduce further (e.g. $75 \%$ reduction compared to baseline) or maintain their current level of reduced smoking. Those who did not reach a reduction of at least $50 \%$ were encouraged to continue working on the $50 \%$ reduction goal. From 3 months onwards, all participants were encouraged to consider cessation, regardless of the reduction achieved at that point.

Outcomes Definition of abstinence: prolonged (from $6-18 \mathrm{~m}$ )

Longest follow-up: $18 \mathrm{~m}$

\begin{tabular}{ll}
\hline Starting date & 01 June 2010 \\
\hline Contact information & Marcel Pieterse PhD \\
\hline Funding source & Netherlands Lung Foundation (grant number 3.4.08.036) \\
\hline Author conflicts of interest & "The authors declare that they have no competing interests" \\
\hline Notes & Relevant comparisons: \\
\hline
\end{tabular}

NCT02124187

Trial name or title Smoking Cessation And Reduction in Depression (SCARID)

Methods Study design: RCT

Location: Italy

Setting: not reported

Recruitment: not reported

Participants Goal N $=129$


NCT02124187 (Continued)

Specialist population?: People with depression

Eligibility criteria: Inclusion: Major Depressive Disorder (MDD) (according to DSM 5 criteria); smoke $\geq 10$ factory-made tobacco cig/day, for at least the past 5 years; age 18 - 65 years; in good general health (absence of cancer, acute myocardial infarction, unstable angina, severe cardiac arrhythmia, recent cerebrovascular incident, or severe atherosclerosis); not currently attempting to quit smoking or wishing to do so in the next 30 days; $6 \mathrm{~m}$ committed to follow the trial procedures Exclusion: use of smokeless tobacco, NRT or other smoking cessation therapies; pregnancy or breastfeeding; current or recent (less than $1 \mathrm{yr}$ ) past history of alcohol and/or drug abuse; active suicidal intention; other significant co-morbidities according to the Investigator's clinical assessment.

\begin{tabular}{ll}
\hline Interventions & Comparator 1: Nicotine-free inhalator \\
& Comparator 2: $0 \mathrm{mg}$ nicotine electronic cigarette \\
& Intervention : $24 \mathrm{mg}$ nicotine electronic cigarette \\
\hline Outcomes & Definition of abstinence: continuous, validated by exhaled CO \\
\hline Starting date & Longest follow-up: $12 \mathrm{~m}$ \\
\hline Contact information & December 2020 \\
\hline Funding source & Pasquale Caponnetto; Giuseppe Minutolo \\
\hline Author conflicts of interest & Not reported \\
\hline Notes & Rot reported \\
\hline
\end{tabular}

\section{NCT02354872}

\begin{tabular}{ll} 
Trial name or title & Motivation project: testing intervention components for the smoker who is unwilling to quit \\
\hline Methods & Study design: Factorial RCT $(2 \times 2 \times 2 \times 2)$ \\
& Location: USA \\
& Setting: Primary care \\
& Recruitment: not reported \\
\hline
\end{tabular}

Participants

Goal N $=577$

Specialist population?: no

Eligibility criteria: Inclusion: age $\geq 18$ years; smoking > 4 cigarettes/day for the previous 6 months; able to read, write, and speak English; have reliable phone access and agree to respond to IVR phone prompts; and if currently using NRT, agreeing to use only study medication for the duration of the study; not currently attempting to quit smoking; not intending to quit smoking (defined as no plans to quit in the next month); and planning to remain in the intervention catchment area for at least 12 months

Exclusion: currently taking bupropion or varenicline; medical contraindications to using NRT including hospitalised (for at least 1 night) for a stroke, heart attack, congestive heart failure or diabetes in the last 30 days; diagnosis of or treatment for schizophrenia, a psychotic disorder or bipolar disorder in the last 10 years; and, if the participant is a woman of childbearing potential, being 
NCT02354872 (Continued) pregnant or intending to becoming pregnant or unwillingness to use an approved method of birth control during treatment

Interventions Participants randomised to 1 of 2 levels on 4 different factors, resulting in 16 trial arms: 1) Nicotine mini-lozenge vs. no mini-lozenge, 2) Behavioral reduction counseling (intervention: BR) vs. no behavioral reduction counseling, 3) 5Rs motivation counseling (intervention 5 R's) vs. no 5Rs motivation counseling, and 4) Behavioral activation counseling (intervention BA) vs. no behavioral activation counseling

Comparator: No behavioural reduction counselling (but participants are also randomised to the treatments above)

Intervention: Behavioral reduction counseling, delivered in 10 (10 - 15 minute) sessions over a 52week period, with an in-person session at Visit 1 followed by 9 phone counselling sessions. Sessions front-loaded to enhance acquisition of new behaviours. Counselling emphasises the development of smoking control skills through feasible, specific, and graded assignments of smoking reduction activities that are tracked over time. Rationales for the reduction intervention, why reduction (e.g. eliminating smoking contexts) should help the smoker, and specific exercises and goals will be provided

Outcomes Definition of abstinence: 7-day point prevalence; validated by exhaled CO

Longest follow-up: $12 \mathrm{~m}$

\begin{tabular}{ll}
\hline Starting date & January 2015 \\
\hline Contact information & $\begin{array}{l}\text { Principal Investigator: Robin Mermelstein, PhD (Institute for Health Research and Policy, University } \\
\text { of Illinois at Chicago) }\end{array}$ \\
\hline Funding source & Not reported \\
\hline Author conflicts of interest & Not reported \\
\hline Notes & $\begin{array}{l}\text { Relevant comparisons: 1) Reduction versus no treatment 2) Reduction versus abrupt; 3) Reduction } \\
\text { method versus reduction method }\end{array}$ \\
\hline
\end{tabular}

\section{NCT02370147}

\begin{tabular}{ll}
\hline Trial name or title & Very brief smoking reduction intervention \\
\hline Methods & Study design: RCT \\
& Location: China \\
& Setting: hospital outpatient clinics \\
& Recruitment: people who smoke and are attending outpatient clinics in the endocrinology and \\
acupuncture departments in a hospital will be enrolled
\end{tabular}

Participants Goal N $=500$

Specialist population?: people attending outpatient clinics in the endocrinology and acupuncture departments for various reasons

Eligibility criteria: Inclusion: aged $\geq 18$ years old; smoking > $10 \mathrm{cpd}$ in past month; no intention to quit smoking; happy to participate in follow-ups, and provide informed consent. 
NCT02370147 (Continued)

Exclusion: $<10 \mathrm{cpd}$ in past month; disease that would make it unethical to tell someone not to quit immediately; cognitively impaired; pregnant

Interventions

Comparator: exercise- and diet-advice group (EDA) (not smoking treatment): counsellors will give participants very brief advice for about 1 minute, advising them to engage in regular physical activity 3 or 4 times a week and to have a healthy and balanced diet including more fruits and vegetables. The tobacco-use status of each smoker will be assessed at each follow-up interview, similar to the intervention group. Smoking cessation or smoking reduction will not be mentioned

Intervention: Smoking-reduction intervention group (SRI): trained counsellors will give participants a very brief smoking-reduction intervention lasting approximately 1 minute. They will be warned of the health problems associated with smoking and advised to reduce smoking consumption to at least half of their total consumption within the next month. Participants will be asked to bear in mind that the current attempt to reduce smoking would be an intermediate step before complete cessation. Additional smoking cessation interventions will be provided at each follow-up

Outcomes Definition of abstinence: prolonged, validated by exhaled CO and salivary cotinine

Longest follow-up: $12 \mathrm{~m}$

\begin{tabular}{ll}
\hline Starting date & March 2015 \\
\hline Contact information & Yao He (Institute of Geriatrics, Chinese PLA General Hospital) \\
\hline Funding source & $\begin{array}{l}\text { The National Natural Science Foundation of China (81373080), the Beijing Municipal Science and } \\
\text { Technology Commission (Z121107001012070) and the Chinese PLA General Hospital (2013FC- } \\
\text { TSYS-1021 and MJ201447) }\end{array}$ \\
\hline
\end{tabular}

Author conflicts of interest $\quad$ "All authors declare that they have no competing interests"

Notes Relevant comparisons: 1) Reduction versus no treatment

\section{NCT02515500}

\begin{tabular}{ll}
\hline Trial name or title & Cigarette reduction using the Quitbit digital lighter and mobile application for smoking cessation \\
\hline Methods & Study design: RCT \\
& Location: USA \\
& Setting: not reported \\
& Recruitment: not reported \\
\hline
\end{tabular}

Goarticipants $\mathrm{N}=200$
Specialist population?: no
$\begin{aligned} & \text { Eligibility criteria: Inclusion: } 18 \text { years of age; cigarette smoker; wanting to quit in the next } 30 \text { days; } \\ & \text { preference to quit gradually (vs abruptly) or having no preference; access to an iPhone (iOS only); } \\ & \text { completion of a run-in period }\end{aligned}$

Exclusion: no major change in number of $\mathrm{cpd}$ in the past month $( \pm 20 \%)$; no major health diagnoses that could impact study attrition

Interventions

Comparator: Gradual cessation: details unclear 
NCT02515500 (Continued)

Intervention: Gradual cessation with Quitbit - cigarette reduction intervention using a novel device called the Quitbit, a digital lighter paired with a smart phone mobile application, to enhance selfregulatory techniques for reducing cpd toward cessation

Outcomes Definition of abstinence: validated by salivary cotinine

Longest follow-up: 6 m

\begin{tabular}{ll}
\hline Starting date & September 2015 \\
\hline Contact information & Julie Wang, PhD, MPH \\
\hline Funding source & Not reported \\
\hline Author conflicts of interest & Not reported \\
\hline Notes & Relevant comparisons: Reduction method versus reduction method (computerised device) \\
\hline
\end{tabular}

\section{NCT02564315}

Trial name or title

The long-term quitting (smoking cessation) study

Methods

Study design: RCT (factorial element, described as sequential, multiple assignment, randomised trial (SMART) design)

Location: USA

Setting: not reported

Recruitment: not reported

\section{Participants}

Goal N = 1157

Specialist population?: no

Eligibility criteria: Inclusion: age $\geq 18$ years; smoking $>4$ cpd for the previous 6 months; able to read, write, and speak English; have reliable phone access and agree to respond to IVR phone prompts; if currently using NRT, agreeing to use only study medication for the duration of the study; motivation to quit smoking; planning to remain in the intervention catchment area for at least 2 years and 2 months

Exclusion: currently taking bupropion or varenicline; unwillingness to cease other forms of nicotine replacement or Chantix (also called varenicline); medical contraindications to using NRT; diagnosis of or treatment for schizophrenia, a psychotic disorder or bipolar disorder in the last 10 years; being pregnant or intending to becoming pregnant or unwillingness to use an approved method of birth control during treatment

Interventions

All participants offered evidence-based cessation treatment (cessation medication plus counselling). Participants who relapse will be eligible to proceed to the next phase and receive 1 of the following treatments:

Comparator 1: Recycling counselling: participants encouraged to quit again as soon as possible. 8 weeks of combination nicotine replacement therapy (nicotine patch + nicotine mini-lozenge)

Comparator 2: Preparation phase control: continuation of Quit Phase treatment plus advice to seek additional help from the Wisconsin Tobacco Quit Line or their clinic care provider

Intervention: Behavioural reduction counselling + nicotine mini-lozenge: targets smoking reduction and preparation for a new quit attempt. 
NCT02564315 (Continued)

Participants who elect to make a new quit attempt will be randomised to 1 of 4 treatment conditions in a $2 \times 2$ fully-crossed factorial design: (a) supportive counseling + skill training; (b) supportive counseling + brief information; (c) skill training + brief information; and (d) brief information only

Outcomes Definition of abstinence: 7-day point prevalence, validated by exhaled CO

Longest follow-up: $14 \mathrm{~m}$

\begin{tabular}{ll}
\hline Starting date & October 2015 \\
\hline Contact information & Tanya Schlam, PhD (University of Wisconsin, Madison) \\
\hline Funding source & Not reported \\
\hline Author conflicts of interest & Not reported \\
\hline Notes & Relevant comparisons: 1 ) Reduction versus abrupt; 2 ) Reduction method versus reduction method \\
\hline
\end{tabular}

\section{NCT02894957}

Trial name or title

Varenicline for "gradual" vs "abrupt" smoking cessation in low-motivated COPD smokers

Study design: RCT
Location: Israel
Setting: pulmonary outpatient clinic
Recruitment: from those attending the outpatient clinic of Pulmonary Institute of the Share Zedek
Medical Center, in Jerusalem
Goal $\mathrm{N}=250$
Specialist population?: people with COPD
Eligibility criteria: Inclusion: men or women aged $\geq 35$ years; currently smoking 10 cpd or more;
having smoked 15 pack years or more; presenting a CO level in expired air $\geq 10$ ppm; with low mo-
tivation to quit; willing to sign a statement of informed consent; willing to sign a written commit-
ment to quit at a target quit date; women of child-bearing potential should agree to use acceptable
contraception methods.
Exclusion: history of treatment with systemic corticosteroids or hospitalisation for a coPD exac-
erbation in the 4-week period prior to enrolment; diagnosis of depression or current treatment
with antidepressants; history of serious psychiatric disorder; myocardial infarction within the last 3
months; unstable angina; severe cardiac arrhythmia; use of any form of smokeless tobacco or nico-
tine substitution or having followed any cessation programme in the past 3 months; alcohol or oth-
er drug addiction; pregnant or lactating women

Interventions

Comparator: Abrupt: participants will be asked to smoke as usual for 6 weeks after enrolment, then stop altogether. Varenicline will be provided post-quit for 12 weeks

Intervention: Gradual: participants will be advised to reduce their smoking by $25 \%$ in the first 2 weeks, $50 \%$ in weeks $3-4$, and $75 \%$ in weeks 5 - 6 ; however, this will be given only as an indication and every participant will be allowed to choose their own goal and rate of progress. To achieve reduction each participant will be offered 3 structured ways: a) scheduled reduction (SR), i.e. gradually increasing the time between cigarettes (the ICl); b) Hierarchical reduction - easiest first, i.e. rating cigarettes in terms of how difficult it would be to give up, then eliminate each in turn, starting with the easiest one; and c) Hierarchical reduction - hardest first, this is similar to the previous one 
NCT02894957 (Continued)

but the participant must start with the hardest cigarette to give up first. Varenicline will be provided pre-quit for 6 weeks and post-quit for 12 weeks

\section{Outcomes}

Definition of abstinence: prolonged abstinence from week 10 to week 30; validated by exhaled CO

Longest follow-up: 30 weeks

\begin{tabular}{ll}
\hline Starting date & June 2019 \\
\hline Contact information & Abraham Bohadana; Gabriel lzbicki \\
\hline Funding source & Not reported \\
\hline Author conflicts of interest & Not reported \\
\hline Notes & Relevant comparisons: 1 ) Reduction versus abrupt \\
\hline
\end{tabular}

\section{NCT02905656}

\begin{tabular}{ll}
\hline Trial name or title & Strategies to promote cessation in smokers who are not ready to quit (PACE) \\
\hline Methods & Study design: Factorial RCT \\
& Location: USA \\
& Setting: not reported \\
& Recruitment: not reported
\end{tabular}

Goal N = 828
Specialist population?: no
Eligibility criteria: Inclusion: able to understand English; for the past 12 months, has smoked 5 or
more cigarettes a day; 18 years or older; planning on quitting smoking someday; access to a tele-
phone; willing and able to use nicotine gum; not currently using Chantix or Wellbutrin
Exclusion: planning to quit smoking cigarettes in the next 30 days; currently pregnant, breastfeed-
ing, or planning to become pregnant in the next 12 months; currently using Chantix or Wellbutrin;
diagnosed with an unstable heart condition

Interventions

Comparator 1: Brief advice: participants receive brief advice to quit smoking, and are provided psycho-education citing health consequences and the positive impact on mortality and morbidity

Comparator 2: Motivational Interviewing (MI): a collaborative conversation style for strengthening a person's own motivation and commitment to change. Ml attempts to avoid a confrontational style and instead guides participants toward choosing to make a change in their behaviour

Intervention 1: Rate reduction: participants will be informed of evidence that systematic reductions in smoking behaviour can lead to long-term smoking cessation. Nicotine gum

Intervention 2: Rate reduction + motivational interviewing (MI): Participants receive both rate reduction and the $\mathrm{MI}$ intervention described above. Nicotine gum

Definition of abstinence: prolonged abstinence (2-week grace period after quitting)
Longest follow-up: $12 \mathrm{~m}$

September 2016 
NCT02905656 (Continued)

\begin{tabular}{ll} 
Contact information & Karen Derefinko; Sarah Hand \\
\hline Funding source & Not reported \\
\hline Author conflicts of interest & Not reported \\
\hline Notes & $\begin{array}{l}\text { Relevant comparisons: } 1 \text { ) Reduction versus abrupt; } 2 \text { ) Reduction method versus reduction method } \\
\text { (MI) }\end{array}$
\end{tabular}

CO: carbon monoxide; COPD: chronic obstructive pulmonary disease; cpd: cigarettes per day; ICI: inter-cigarette interval; IVR: interactive voice response; ppm: parts per million

\section{DATA AND ANALYSES}

\section{Comparison 1. Reduction to quit versus no treatment}

\begin{tabular}{|c|c|c|c|c|}
\hline Outcome or subgroup title & $\begin{array}{l}\text { No. of } \\
\text { studies }\end{array}$ & $\begin{array}{l}\text { No. of } \\
\text { partici- } \\
\text { pants }\end{array}$ & Statistical method & Effect size \\
\hline 1 Abstinence & 6 & 1599 & $\begin{array}{l}\text { Risk Ratio (M-H, Random, 95\% } \\
\mathrm{Cl} \text { ) }\end{array}$ & $1.74[0.90,3.38]$ \\
\hline $\begin{array}{l}2 \text { Abstinence: subgrouped by pre-quit } \\
\text { pharma in reduction arm }\end{array}$ & 6 & & $\begin{array}{l}\text { Risk Ratio (M-H, Random, 95\% } \\
\mathrm{Cl} \text { ) }\end{array}$ & Subtotals only \\
\hline 2.1 Nicotine patch +/or gum & 2 & 633 & $\begin{array}{l}\text { Risk Ratio (M-H, Random, 95\% } \\
\mathrm{Cl} \text { ) }\end{array}$ & $1.64[0.42,6.44]$ \\
\hline 2.2 None & 5 & 966 & $\begin{array}{l}\text { Risk Ratio (M-H, Random, 95\% } \\
\mathrm{Cl} \text { ) }\end{array}$ & $1.64[0.88,3.07]$ \\
\hline 3 Quit attempts & 1 & & $\begin{array}{l}\text { Risk Ratio (M-H, Random, 95\% } \\
\mathrm{Cl} \text { ) }\end{array}$ & Totals not selected \\
\hline 4 Pre-quit reduction in cpd of at least $50 \%$ & 2 & 473 & $\begin{array}{l}\text { Risk Ratio (M-H, Random, 95\% } \\
\mathrm{Cl})\end{array}$ & $1.79[1.28,2.51]$ \\
\hline 5 Pre-quit SAEs & 1 & & $\begin{array}{l}\text { Risk Ratio (M-H, Random, 95\% } \\
\mathrm{Cl} \text { ) }\end{array}$ & Totals not selected \\
\hline
\end{tabular}

Analysis 1.1. Comparison 1 Reduction to quit versus no treatment, Outcome 1 Abstinence.

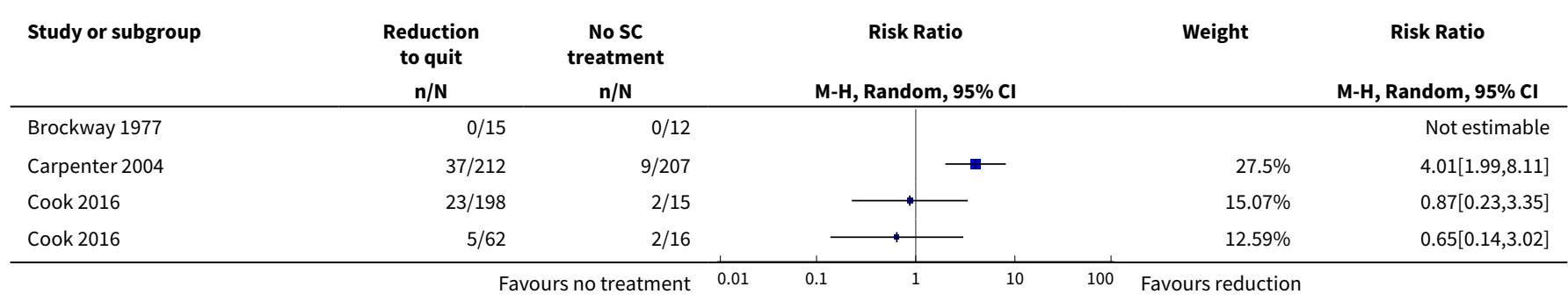




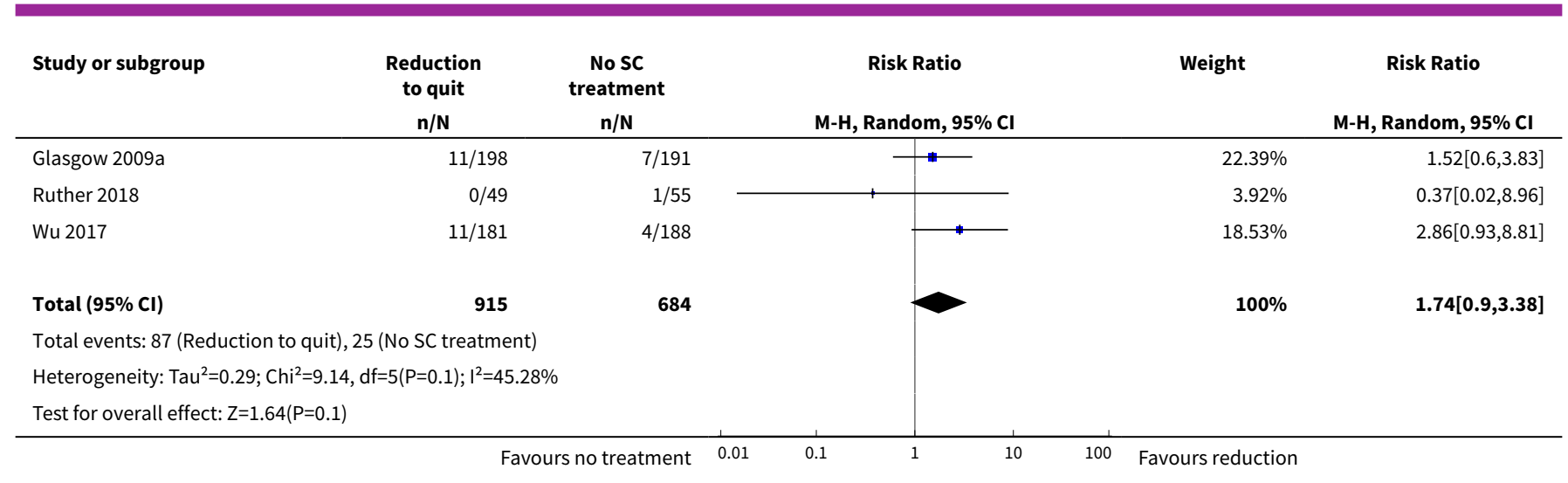

Analysis 1.2. Comparison 1 Reduction to quit versus no treatment, Outcome 2 Abstinence: subgrouped by pre-quit pharma in reduction arm.

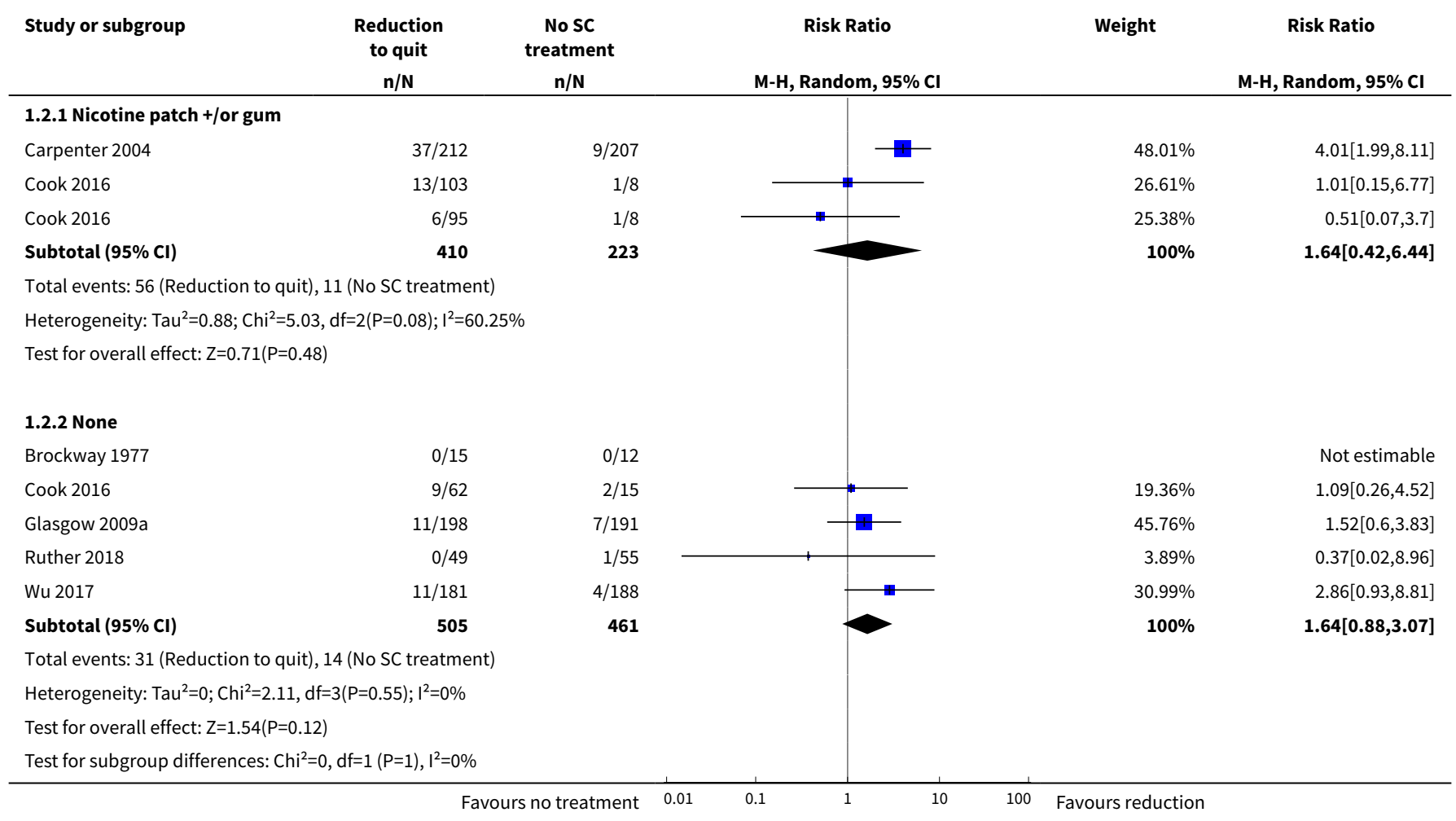

Analysis 1.3. Comparison 1 Reduction to quit versus no treatment, Outcome 3 Quit attempts.

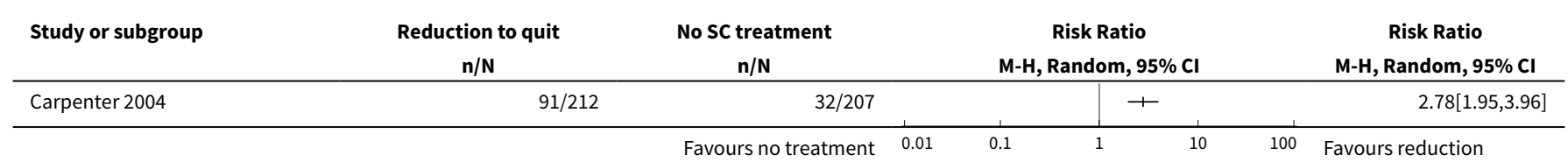




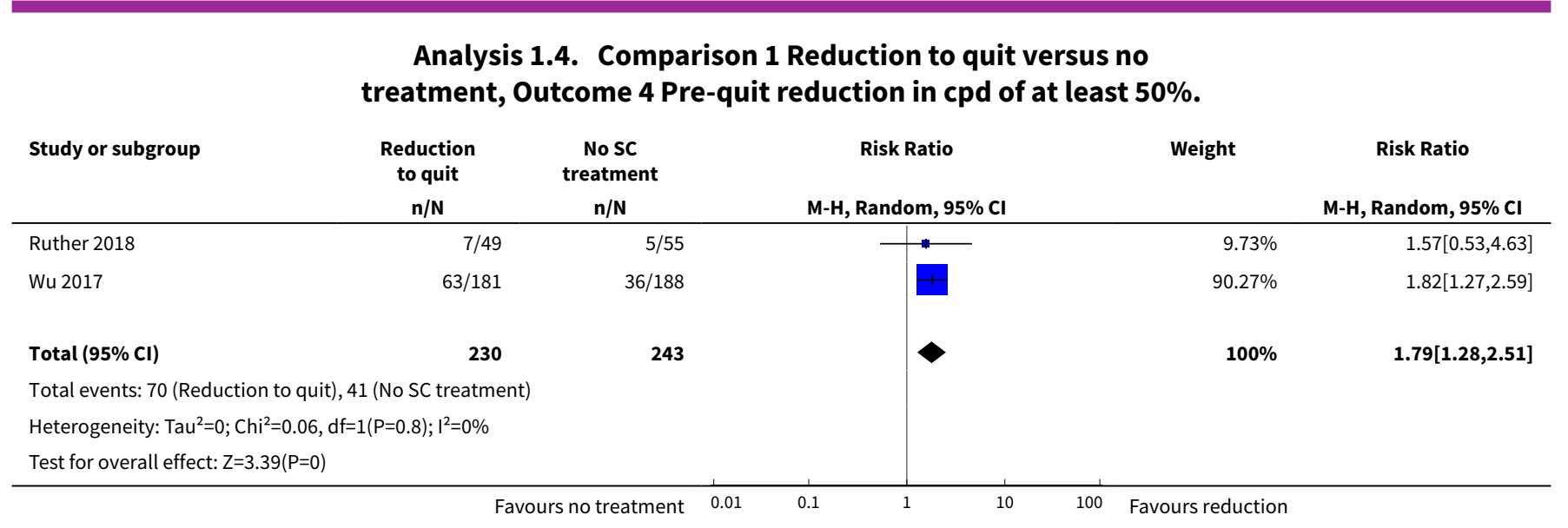

Analysis 1.5. Comparison 1 Reduction to quit versus no treatment, Outcome 5 Pre-quit SAEs.

\begin{tabular}{|c|c|c|c|c|c|c|c|}
\hline Study or subgroup & $\begin{array}{l}\text { Reduction to quit } \\
\mathrm{n} / \mathrm{N} \\
\end{array}$ & $\begin{array}{c}\text { No SC treatment } \\
\mathrm{n} / \mathrm{N} \\
\end{array}$ & & & $\begin{array}{l}\text { k Ratio } \\
\text { dom, } 95 \% \mathrm{Cl}\end{array}$ & & $\begin{array}{c}\text { Risk Ratio } \\
\text { M-H, Random, 95\% Cl }\end{array}$ \\
\hline Cook 2016 & $0 / 260$ & $0 / 31$ & & & & & Not estimable \\
\hline
\end{tabular}

Comparison 2. Reduction to quit versus abrupt quitting

\begin{tabular}{|c|c|c|c|c|}
\hline Outcome or subgroup title & $\begin{array}{l}\text { No. of } \\
\text { studies }\end{array}$ & $\begin{array}{l}\text { No. of } \\
\text { partici- } \\
\text { pants }\end{array}$ & Statistical method & Effect size \\
\hline 1 Abstinence & 22 & 9219 & $\begin{array}{l}\text { Risk Ratio (M-H, Random, 95\% } \\
\text { Cl) }\end{array}$ & $1.01[0.87,1.17]$ \\
\hline $\begin{array}{l}2 \text { Abstinence: sensitivity analysis removing } \\
\text { studies with lower-intensity abrupt arms }\end{array}$ & 17 & 6656 & $\begin{array}{l}\text { Risk Ratio (M-H, Random, 95\% } \\
\text { Cl) }\end{array}$ & $0.95[0.80,1.12]$ \\
\hline $\begin{array}{l}3 \text { Abstinence: subgrouped by pre-quit } \\
\text { pharma in reduction arm }\end{array}$ & 22 & 9219 & $\begin{array}{l}\text { Risk Ratio (M-H, Random, 95\% } \\
\text { Cl) }\end{array}$ & $1.01[0.87,1.17]$ \\
\hline 3.1 Varenicline & 1 & 314 & $\begin{array}{l}\text { Risk Ratio (M-H, Random, 95\% } \\
\text { Cl) }\end{array}$ & $1.48[1.16,1.90]$ \\
\hline 3.2 NRT & 9 & 4359 & $\begin{array}{l}\text { Risk Ratio (M-H, Random, 95\% } \\
\text { Cl) }\end{array}$ & $0.91[0.72,1.16]$ \\
\hline 3.3 None & 13 & 4546 & $\begin{array}{l}\text { Risk Ratio (M-H, Random, 95\% } \\
\text { Cl) }\end{array}$ & $1.01[0.85,1.19]$ \\
\hline 4 Abstinence: subgrouped by set quit date & 22 & 9219 & $\begin{array}{l}\text { Risk Ratio (M-H, Random, 95\% } \\
\text { Cl) }\end{array}$ & $1.01[0.88,1.17]$ \\
\hline 4.1 Quit date set & 14 & 4704 & $\begin{array}{l}\text { Risk Ratio (M-H, Random, 95\% } \\
\mathrm{Cl} \text { ) }\end{array}$ & $0.98[0.81,1.18]$ \\
\hline
\end{tabular}




\begin{tabular}{|c|c|c|c|c|}
\hline Outcome or subgroup title & $\begin{array}{l}\text { No. of } \\
\text { studies }\end{array}$ & $\begin{array}{l}\text { No. of } \\
\text { partici- } \\
\text { pants }\end{array}$ & Statistical method & Effect size \\
\hline 4.2 No quit date set & 6 & 3128 & $\begin{array}{l}\text { Risk Ratio (M-H, Random, 95\% } \\
\mathrm{Cl})\end{array}$ & $1.05[0.78,1.41]$ \\
\hline 4.3 Unclear & 2 & 1387 & $\begin{array}{l}\text { Risk Ratio (M-H, Random, 95\% } \\
\mathrm{Cl})\end{array}$ & $1.16[0.71,1.88]$ \\
\hline $\begin{array}{l}5 \text { Abstinence: subgrouped by cpd vs sfp re- } \\
\text { duction }\end{array}$ & 22 & 9219 & $\begin{array}{l}\text { Risk Ratio (M-H, Random, 95\% } \\
\mathrm{Cl})\end{array}$ & $1.01[0.88,1.17]$ \\
\hline 5.1 Cigarettes per day (cpd) & 14 & 4503 & $\begin{array}{l}\text { Risk Ratio (M-H, Random, 95\% } \\
\mathrm{Cl})\end{array}$ & $1.05[0.89,1.23]$ \\
\hline 5.2 Smoke free periods (sfp) & 1 & 64 & $\begin{array}{l}\text { Risk Ratio (M-H, Random, 95\% } \\
\mathrm{Cl} \text { ) }\end{array}$ & $0.82[0.39,1.70]$ \\
\hline 5.3 Choice of cpd or sfp & 6 & 4513 & $\begin{array}{l}\text { Risk Ratio (M-H, Random, 95\% } \\
\mathrm{Cl})\end{array}$ & $1.01[0.72,1.42]$ \\
\hline 5.4 Unclear & 1 & 139 & $\begin{array}{l}\text { Risk Ratio (M-H, Random, 95\% } \\
\text { Cl) }\end{array}$ & $0.96[0.54,1.70]$ \\
\hline $\begin{array}{l}6 \text { Abstinence: subgrouped by structured vs } \\
\text { unstructured reduction advised }\end{array}$ & 22 & 9219 & $\begin{array}{l}\text { Risk Ratio (M-H, Random, 95\% } \\
\mathrm{Cl})\end{array}$ & $1.01[0.87,1.17]$ \\
\hline 6.1 Structured reduction advice & 16 & 8172 & $\begin{array}{l}\text { Risk Ratio (M-H, Random, 95\% } \\
\mathrm{Cl} \text { ) }\end{array}$ & $0.96[0.81,1.14]$ \\
\hline 6.2 Unstructured reduction advice & 6 & 908 & $\begin{array}{l}\text { Risk Ratio (M-H, Random, 95\% } \\
\mathrm{CI})\end{array}$ & $1.19[0.92,1.54]$ \\
\hline 6.3 Unclear & 1 & 139 & $\begin{array}{l}\text { Risk Ratio (M-H, Random, 95\% } \\
\mathrm{Cl} \text { ) }\end{array}$ & $0.96[0.54,1.70]$ \\
\hline $\begin{array}{l}7 \text { Abstinence: subgrouped by length of the } \\
\text { reduction period }\end{array}$ & 22 & 9219 & $\begin{array}{l}\text { Risk Ratio (M-H, Random, 95\% } \\
\text { Cl) }\end{array}$ & $1.01[0.88,1.17]$ \\
\hline 7.1 Less than or equal to 4 weeks & 13 & 5277 & $\begin{array}{l}\text { Risk Ratio (M-H, Random, 95\% } \\
\mathrm{Cl})\end{array}$ & $0.95[0.81,1.10]$ \\
\hline 7.25 to 13 weeks & 5 & 3266 & $\begin{array}{l}\text { Risk Ratio (M-H, Random, 95\% } \\
\mathrm{Cl} \text { ) }\end{array}$ & $1.01[0.75,1.37]$ \\
\hline 7.36 months & 1 & 100 & $\begin{array}{l}\text { Risk Ratio (M-H, Random, 95\% } \\
\mathrm{Cl} \text { ) }\end{array}$ & $0.25[0.03,2.16]$ \\
\hline 7.418 months & 1 & 152 & $\begin{array}{l}\text { Risk Ratio (M-H, Random, 95\% } \\
\mathrm{Cl})\end{array}$ & $0.95[0.40,2.26]$ \\
\hline 7.5 Unclear & 2 & 424 & $\begin{array}{l}\text { Risk Ratio (M-H, Random, 95\% } \\
\mathrm{Cl})\end{array}$ & $1.24[0.74,2.06]$ \\
\hline $\begin{array}{l}8 \text { Abstinence: subgrouped by reduction } \\
\text { goal }\end{array}$ & 22 & 9219 & $\begin{array}{l}\text { Risk Ratio (M-H, Random, 95\% } \\
\mathrm{Cl} \text { ) }\end{array}$ & $1.01[0.88,1.17]$ \\
\hline
\end{tabular}




\begin{tabular}{|c|c|c|c|c|}
\hline Outcome or subgroup title & $\begin{array}{l}\text { No. of } \\
\text { studies }\end{array}$ & $\begin{array}{l}\text { No. of } \\
\text { partici- } \\
\text { pants }\end{array}$ & Statistical method & Effect size \\
\hline 8.1 Reduce $<50 \%$ & 1 & 91 & $\begin{array}{l}\text { Risk Ratio (M-H, Random, 95\% } \\
\mathrm{Cl} \text { ) }\end{array}$ & $1.21[0.62,2.36]$ \\
\hline 8.2 Reduce $50 \%$ & 4 & 565 & $\begin{array}{l}\text { Risk Ratio (M-H, Random, 95\% } \\
\mathrm{Cl})\end{array}$ & $1.02[0.71,1.46]$ \\
\hline 8.3 Reduce $75-85 \%$ & 3 & 1571 & $\begin{array}{l}\text { Risk Ratio (M-H, Random, 95\% } \\
\mathrm{Cl})\end{array}$ & $0.78[0.57,1.07]$ \\
\hline 8.4 Reduce $100 \%$ & 7 & 1779 & $\begin{array}{l}\text { Risk Ratio (M-H, Random, 95\% } \\
\mathrm{Cl})\end{array}$ & $1.06[0.78,1.44]$ \\
\hline 8.5 Chosen by individual participants & 5 & 3810 & $\begin{array}{l}\text { Risk Ratio (M-H, Random, 95\% } \\
\mathrm{Cl} \text { ) }\end{array}$ & $1.03[0.78,1.37]$ \\
\hline 8.6 No goals stated & 3 & 1403 & $\begin{array}{l}\text { Risk Ratio (M-H, Random, 95\% } \\
\mathrm{Cl})\end{array}$ & $1.14[0.72,1.79]$ \\
\hline 9 Quit attempts & 11 & 5389 & $\begin{array}{l}\text { Risk Ratio (M-H, Random, 95\% } \\
\text { Cl) }\end{array}$ & $0.92[0.85,0.99]$ \\
\hline 10 Pre-quit reduction of at least $50 \%$ & 1 & & $\begin{array}{l}\text { Risk Ratio (M-H, Random, 95\% } \\
\mathrm{Cl})\end{array}$ & Totals not selected \\
\hline $10.1 \mathrm{Cpd}$ & 1 & & $\begin{array}{l}\text { Risk Ratio (M-H, Random, 95\% } \\
\mathrm{Cl} \text { ) }\end{array}$ & $0.0[0.0,0.0]$ \\
\hline 10.2 Exhaled CO & 1 & & $\begin{array}{l}\text { Risk Ratio (M-H, Random, 95\% } \\
\mathrm{Cl})\end{array}$ & $0.0[0.0,0.0]$ \\
\hline 11 Pre-quit reduction in cpd & 5 & & $\begin{array}{l}\text { Mean Difference (IV, Random, } \\
95 \% \mathrm{CI} \text { ) }\end{array}$ & Totals not selected \\
\hline 12 Pre-quit reduction in carbon monoxide & 1 & & $\begin{array}{l}\text { Mean Difference (IV, Random, } \\
95 \% \mathrm{CI})\end{array}$ & Totals not selected \\
\hline 13 Pre-quit reduction in cotinine & 1 & 128 & $\begin{array}{l}\text { Mean Difference (IV, Random, } \\
95 \% \mathrm{CI} \text { ) }\end{array}$ & $95.12[6.60,183.64]$ \\
\hline 14 Pre-quit SAEs & 6 & & $\begin{array}{l}\text { Risk Ratio (M-H, Random, 95\% } \\
\text { Cl) }\end{array}$ & Subtotals only \\
\hline 14.1 NRT & 4 & 1559 & $\begin{array}{l}\text { Risk Ratio (M-H, Random, 95\% } \\
\mathrm{Cl} \text { ) }\end{array}$ & $1.19[0.63,2.27]$ \\
\hline 14.2 No pharmacotherapy & 3 & 750 & $\begin{array}{l}\text { Risk Ratio (M-H, Random, 95\% } \\
\mathrm{Cl})\end{array}$ & $0.0[0.0,0.0]$ \\
\hline $\begin{array}{l}15 \text { Pre-quit tobacco withdrawal \& addition- } \\
\text { al AE information }\end{array}$ & & & Other data & No numeric data \\
\hline $\begin{array}{l}16 \text { Moderation of the reduction vs abrupt } \\
\text { quitting effect }\end{array}$ & & & Other data & No numeric data \\
\hline
\end{tabular}


Analysis 2.1. Comparison 2 Reduction to quit versus abrupt quitting, Outcome 1 Abstinence.

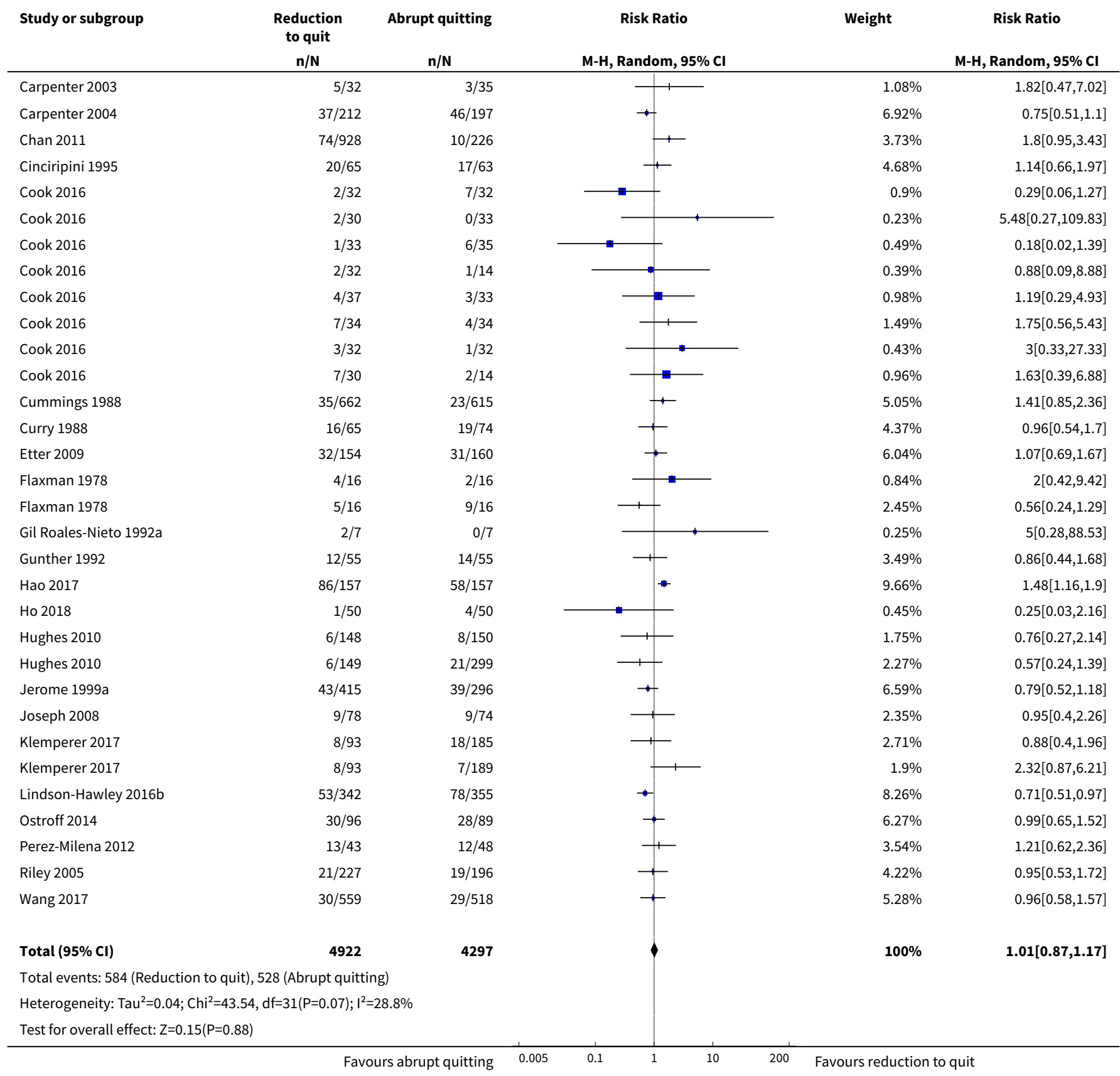


Analysis 2.2. Comparison 2 Reduction to quit versus abrupt quitting, Outcome 2 Abstinence: sensitivity analysis removing studies with lower-intensity abrupt arms.

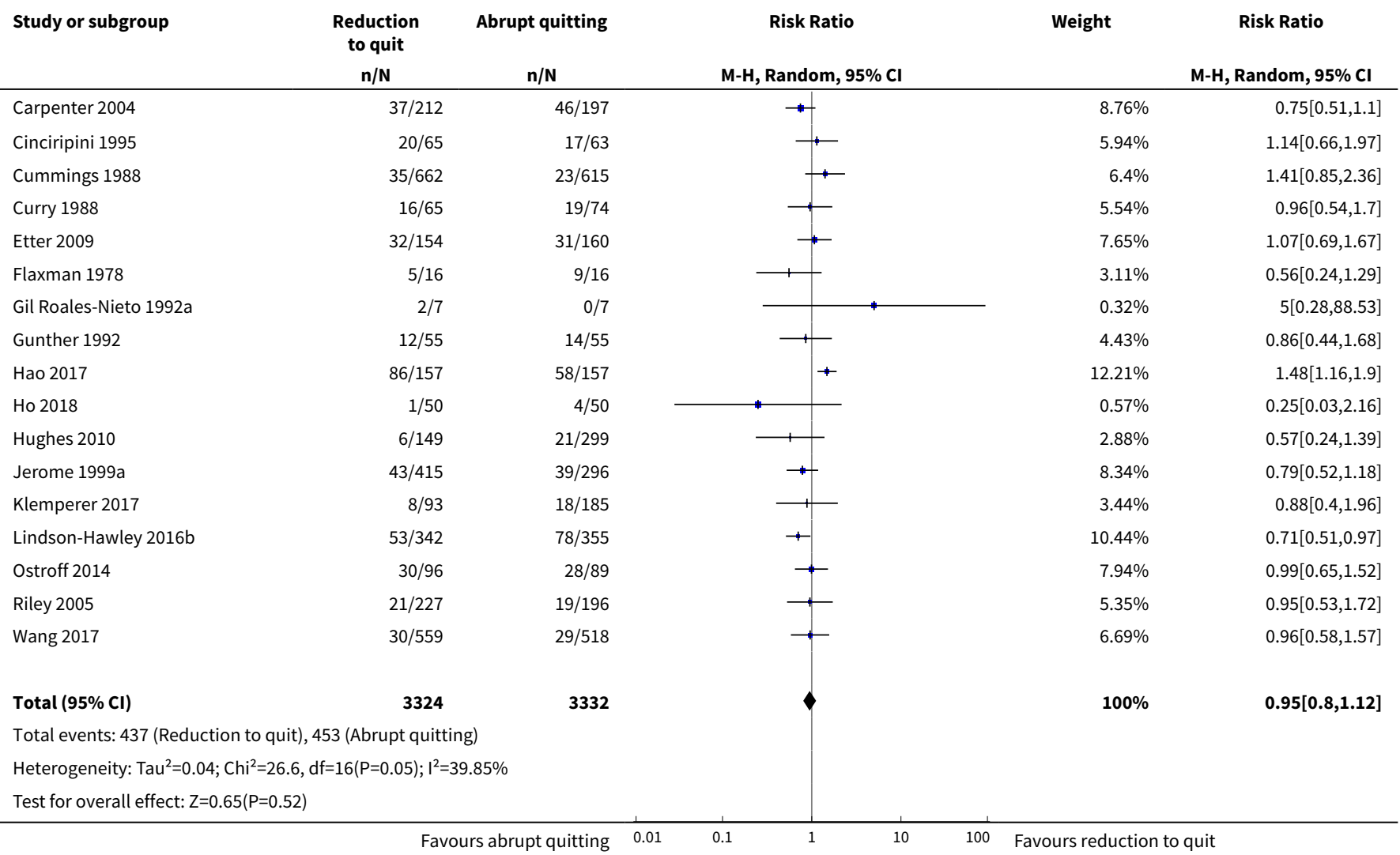

Analysis 2.3. Comparison 2 Reduction to quit versus abrupt quitting, Outcome 3 Abstinence: subgrouped by pre-quit pharma in reduction arm.

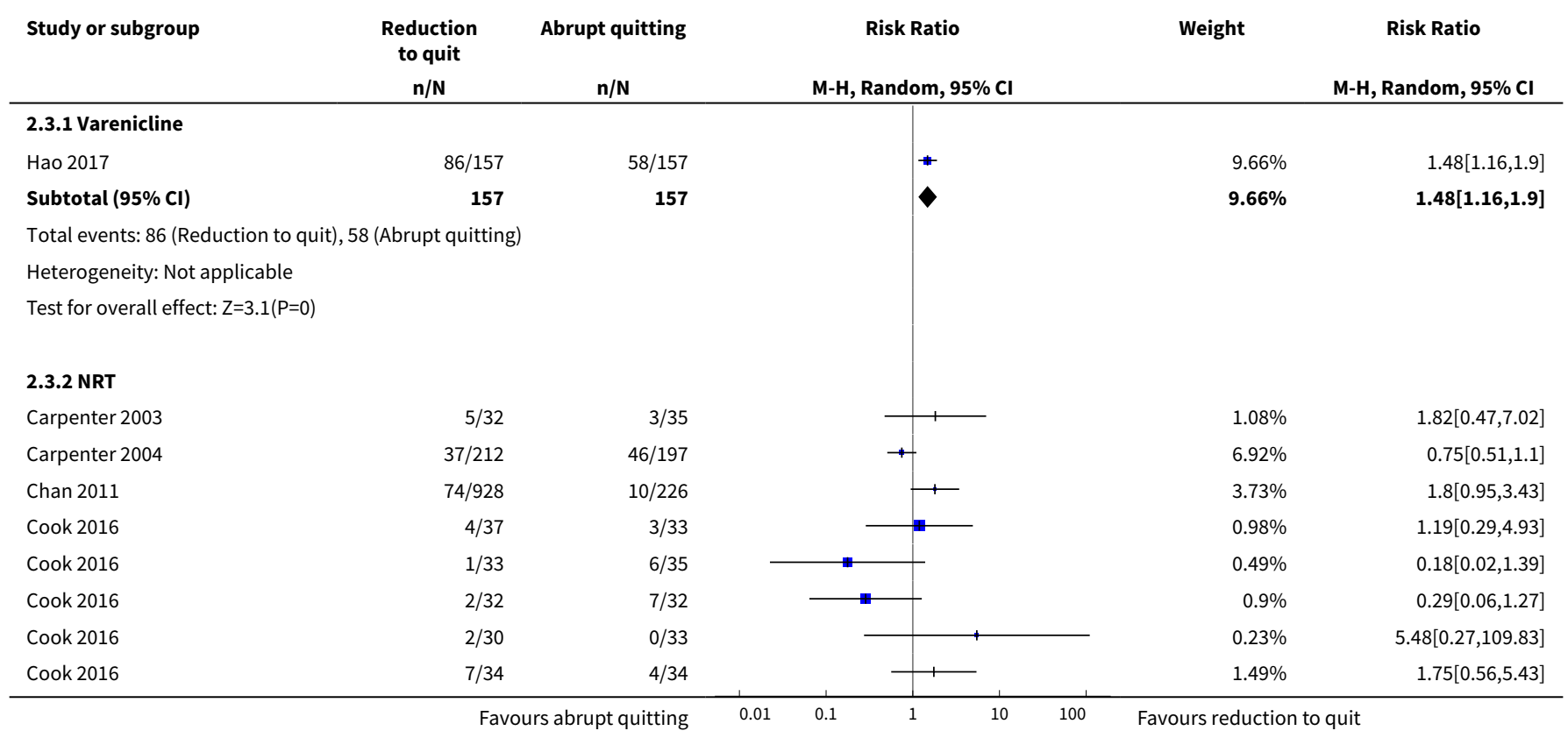




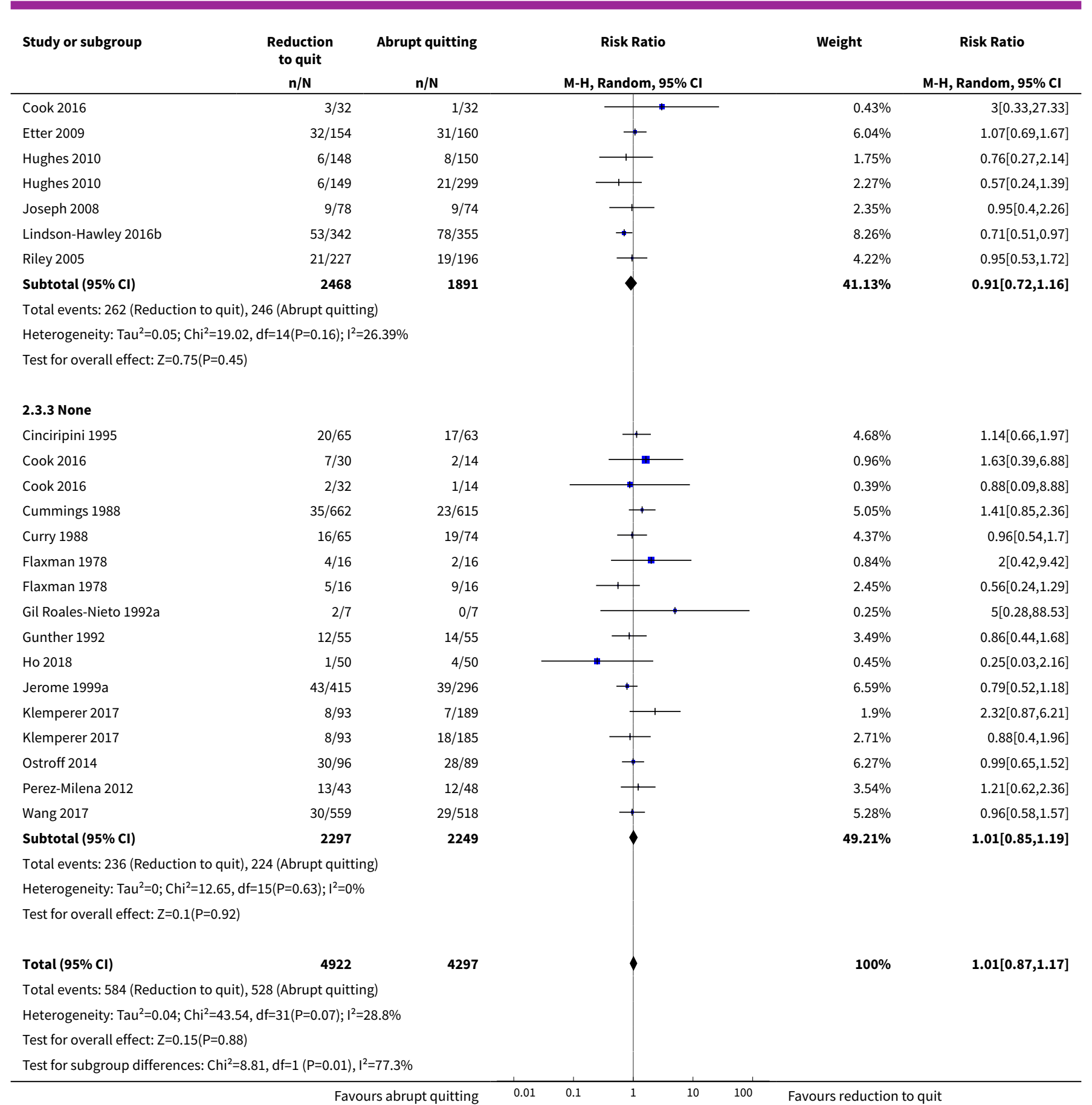

Analysis 2.4. Comparison 2 Reduction to quit versus abrupt quitting, Outcome 4 Abstinence: subgrouped by set quit date.

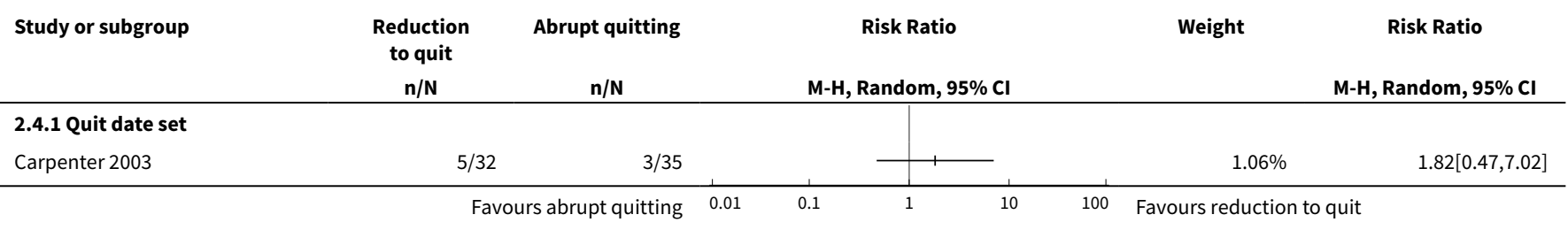




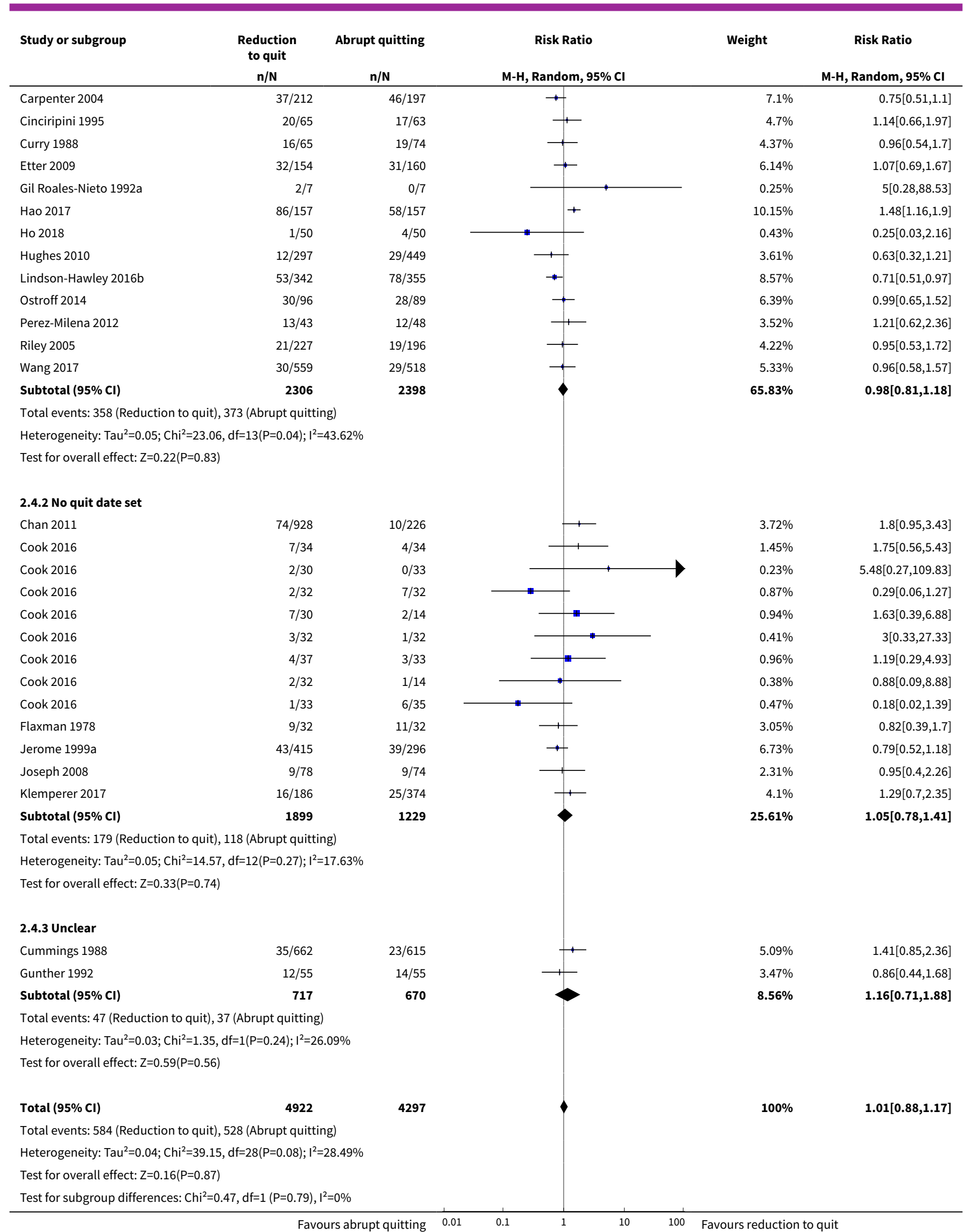


Analysis 2.5. Comparison 2 Reduction to quit versus abrupt quitting, Outcome 5 Abstinence: subgrouped by cpd vs sfp reduction.

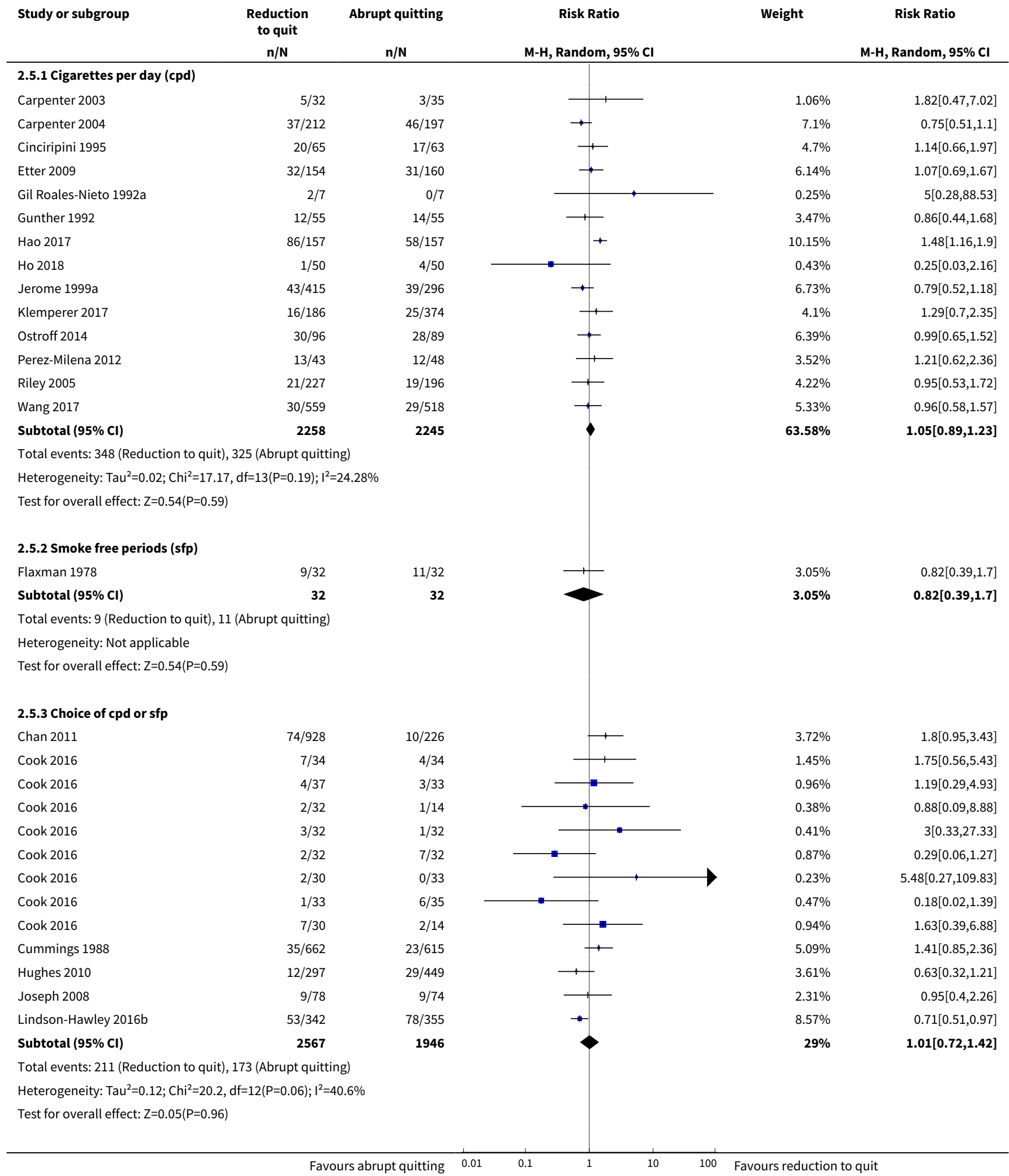




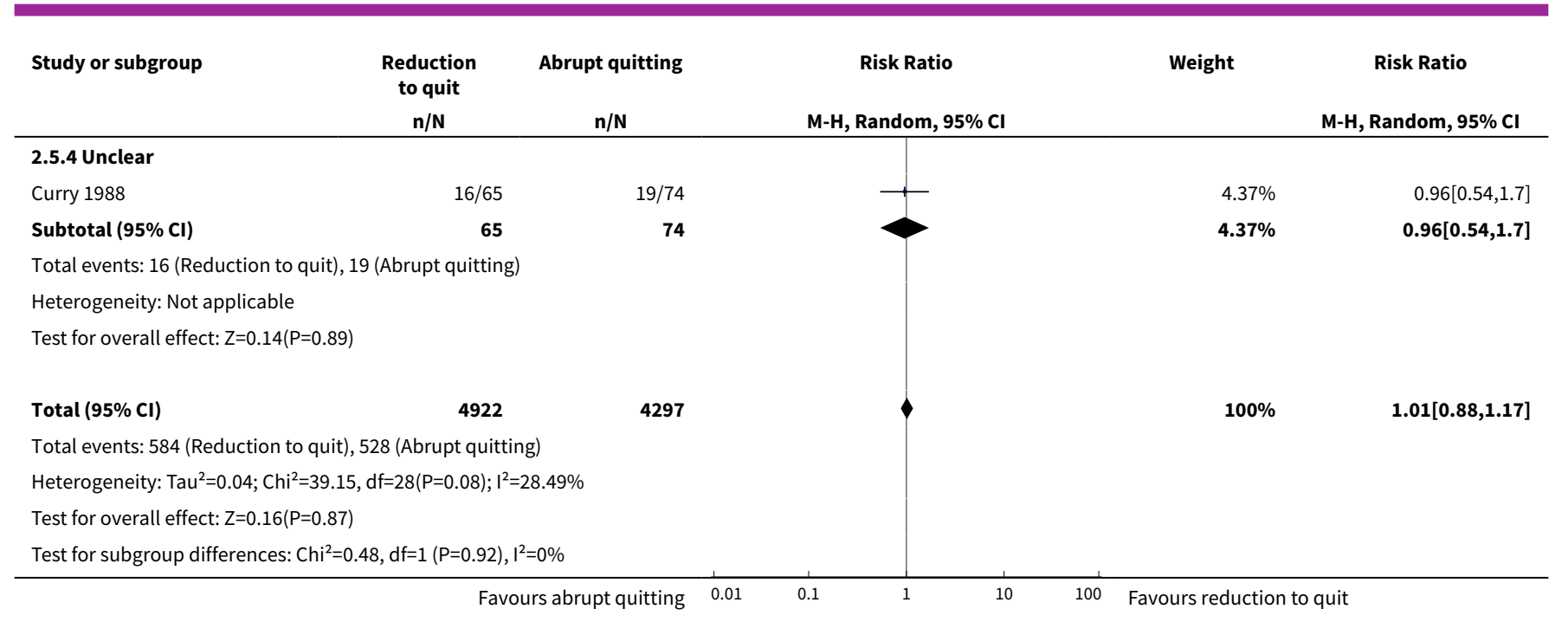

Analysis 2.6. Comparison 2 Reduction to quit versus abrupt quitting, Outcome 6 Abstinence: subgrouped by structured vs unstructured reduction advised.

\begin{tabular}{|c|c|c|c|c|c|}
\hline Study or subgroup & $\begin{array}{l}\text { Reduction } \\
\text { to quit } \\
n / N\end{array}$ & $\begin{array}{l}\text { Abrupt quitting } \\
\qquad \mathrm{n} / \mathrm{N}\end{array}$ & Risk Ratio & Weight & $\begin{array}{c}\text { Risk Ratio } \\
\text { M-H, Random, } 95 \% \mathrm{CI}\end{array}$ \\
\hline \multicolumn{6}{|c|}{ 2.6.1 Structured reduction advice } \\
\hline Carpenter 2003 & $5 / 32$ & $3 / 35$ & \begin{tabular}{l|l} 
& 1
\end{tabular} & $1.1 \%$ & $1.82[0.47,7.02]$ \\
\hline Carpenter 2004 & $37 / 212$ & $46 / 197$ & $\rightarrow$ & $6.93 \%$ & $0.75[0.51,1.1]$ \\
\hline Chan 2011 & $74 / 928$ & $10 / 226$ & -1 & $3.76 \%$ & $1.8[0.95,3.43]$ \\
\hline Cook 2016 & $7 / 30$ & $2 / 14$ & \begin{tabular}{l|l} 
\\
\end{tabular} & $0.98 \%$ & $1.63[0.39,6.88]$ \\
\hline Cook 2016 & $7 / 34$ & $4 / 34$ & 1 & $1.5 \%$ & $1.75[0.56,5.43]$ \\
\hline Cook 2016 & $1 / 33$ & $6 / 35$ & - & $0.49 \%$ & $0.18[0.02,1.39]$ \\
\hline Cook 2016 & $2 / 32$ & $7 / 32$ & + & $0.91 \%$ & $0.29[0.06,1.27]$ \\
\hline Cook 2016 & $4 / 37$ & $3 / 33$ & 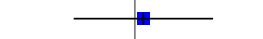 & $1 \%$ & $1.19[0.29,4.93]$ \\
\hline Cook 2016 & $2 / 30$ & $0 / 33$ & & $0.24 \%$ & $5.48[0.27,109.83]$ \\
\hline Cook 2016 & $2 / 32$ & $1 / 14$ & & $0.39 \%$ & $0.88[0.09,8.88]$ \\
\hline Cummings 1988 & $35 / 662$ & $23 / 615$ & 1 & $5.07 \%$ & $1.41[0.85,2.36]$ \\
\hline Flaxman 1978 & $9 / 32$ & $11 / 32$ & 1 & $3.11 \%$ & $0.82[0.39,1.7]$ \\
\hline Ho 2018 & $1 / 50$ & $4 / 50$ & \begin{tabular}{l|l} 
\\
\end{tabular} & $0.45 \%$ & $0.25[0.03,2.16]$ \\
\hline Hughes 2010 & $12 / 297$ & $29 / 449$ & 1 & $3.66 \%$ & $0.63[0.32,1.21]$ \\
\hline Jerome 1999a & $43 / 415$ & $39 / 296$ & $\rightarrow$ & $6.6 \%$ & $0.79[0.52,1.18]$ \\
\hline Joseph 2008 & $9 / 78$ & $9 / 74$ & 1 & $2.37 \%$ & $0.95[0.4,2.26]$ \\
\hline Klemperer 2017 & $16 / 186$ & $25 / 374$ & 1 & $4.13 \%$ & $1.29[0.7,2.35]$ \\
\hline Lindson-Hawley 2016b & $53 / 342$ & $78 / 355$ & $\rightarrow$ & $8.24 \%$ & $0.71[0.51,0.97]$ \\
\hline Ostroff 2014 & $30 / 96$ & $28 / 89$ & $\rightarrow$ & $6.28 \%$ & $0.99[0.65,1.52]$ \\
\hline Riley 2005 & $21 / 227$ & $19 / 196$ & $\longrightarrow$ & $4.24 \%$ & $0.95[0.53,1.72]$ \\
\hline Wang 2017 & $30 / 559$ & $29 / 518$ & $\rightarrow$ & $5.3 \%$ & $0.96[0.58,1.57]$ \\
\hline Subtotal $(95 \% \mathrm{CI})$ & 4408 & 3764 & $\vec{\gamma}$ & $70.41 \%$ & $0.96[0.81,1.14]$ \\
\hline \multicolumn{6}{|c|}{ Test for overall effect: $Z=0.44(P=0.66)$} \\
\hline
\end{tabular}




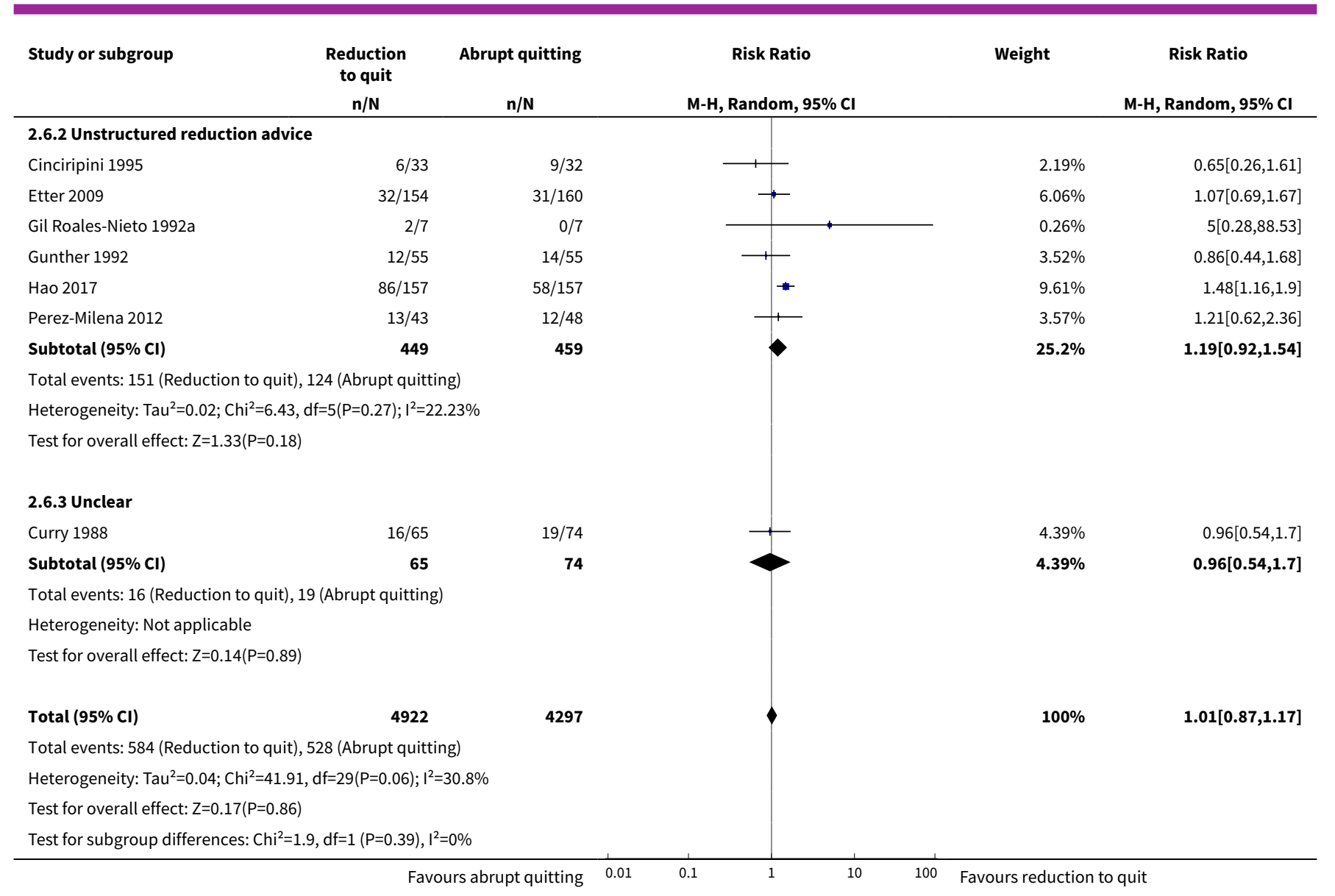

Analysis 2.7. Comparison 2 Reduction to quit versus abrupt quitting, Outcome 7 Abstinence: subgrouped by length of the reduction period.

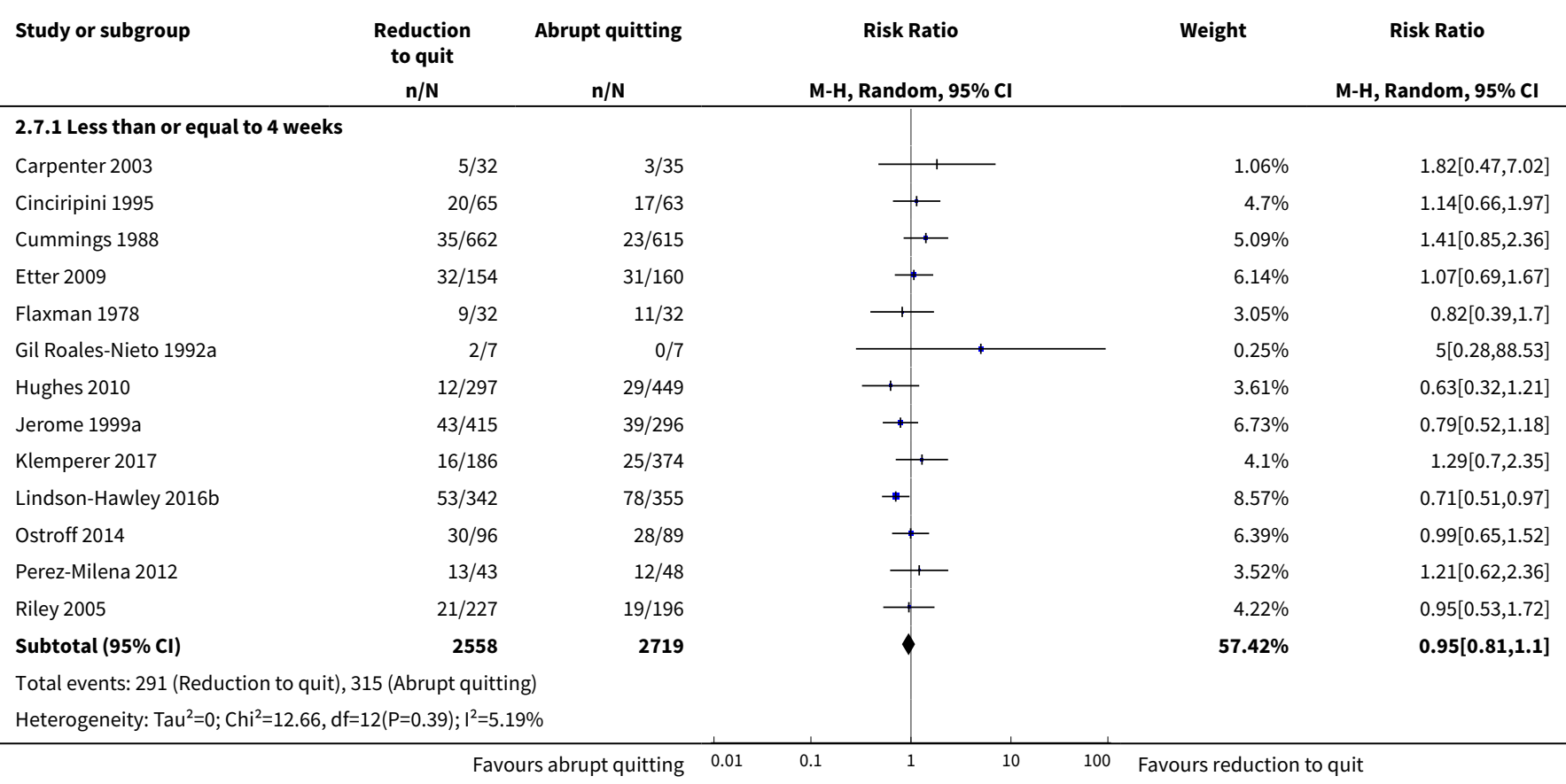




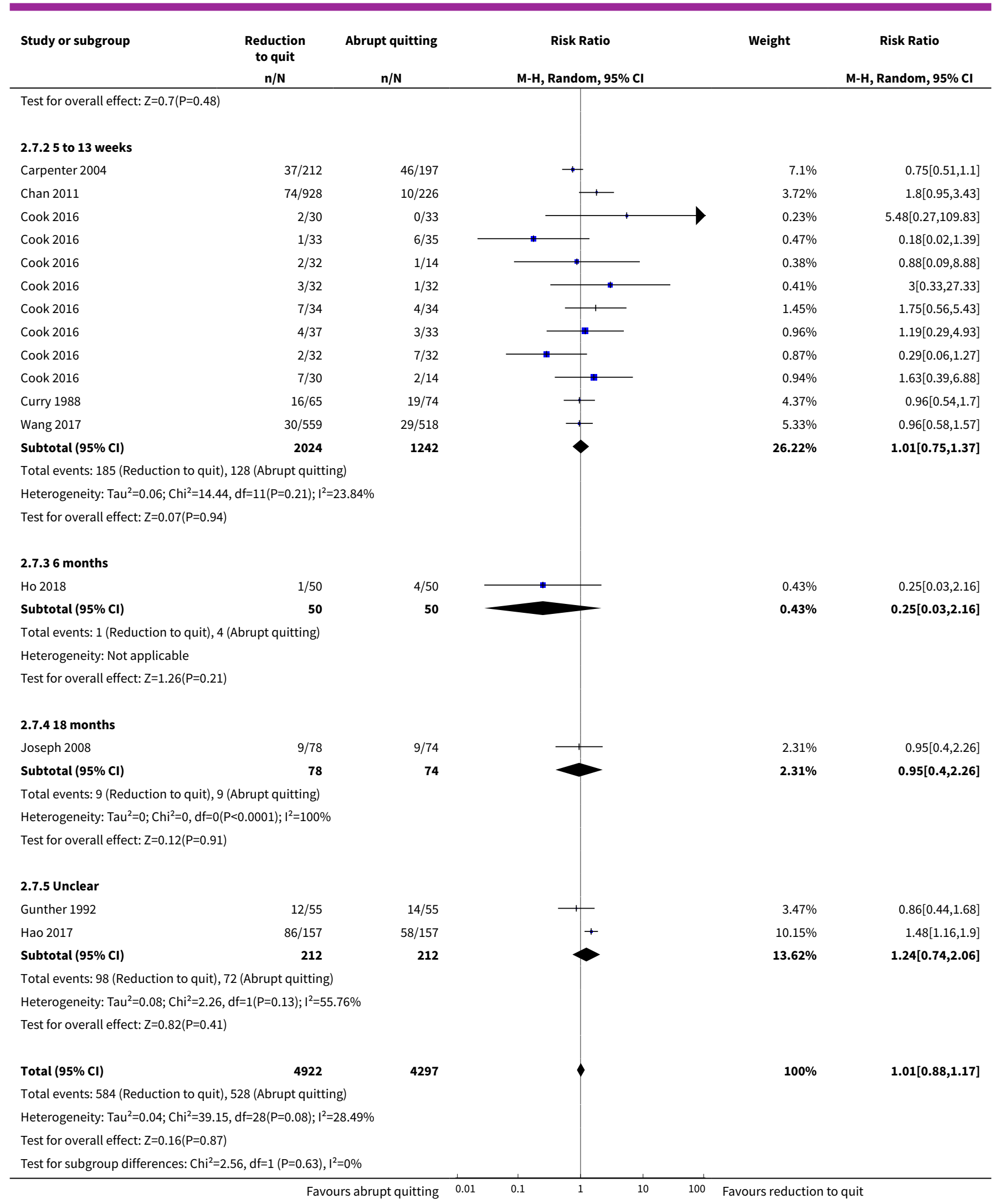


Analysis 2.8. Comparison 2 Reduction to quit versus abrupt quitting, Outcome 8 Abstinence: subgrouped by reduction goal.

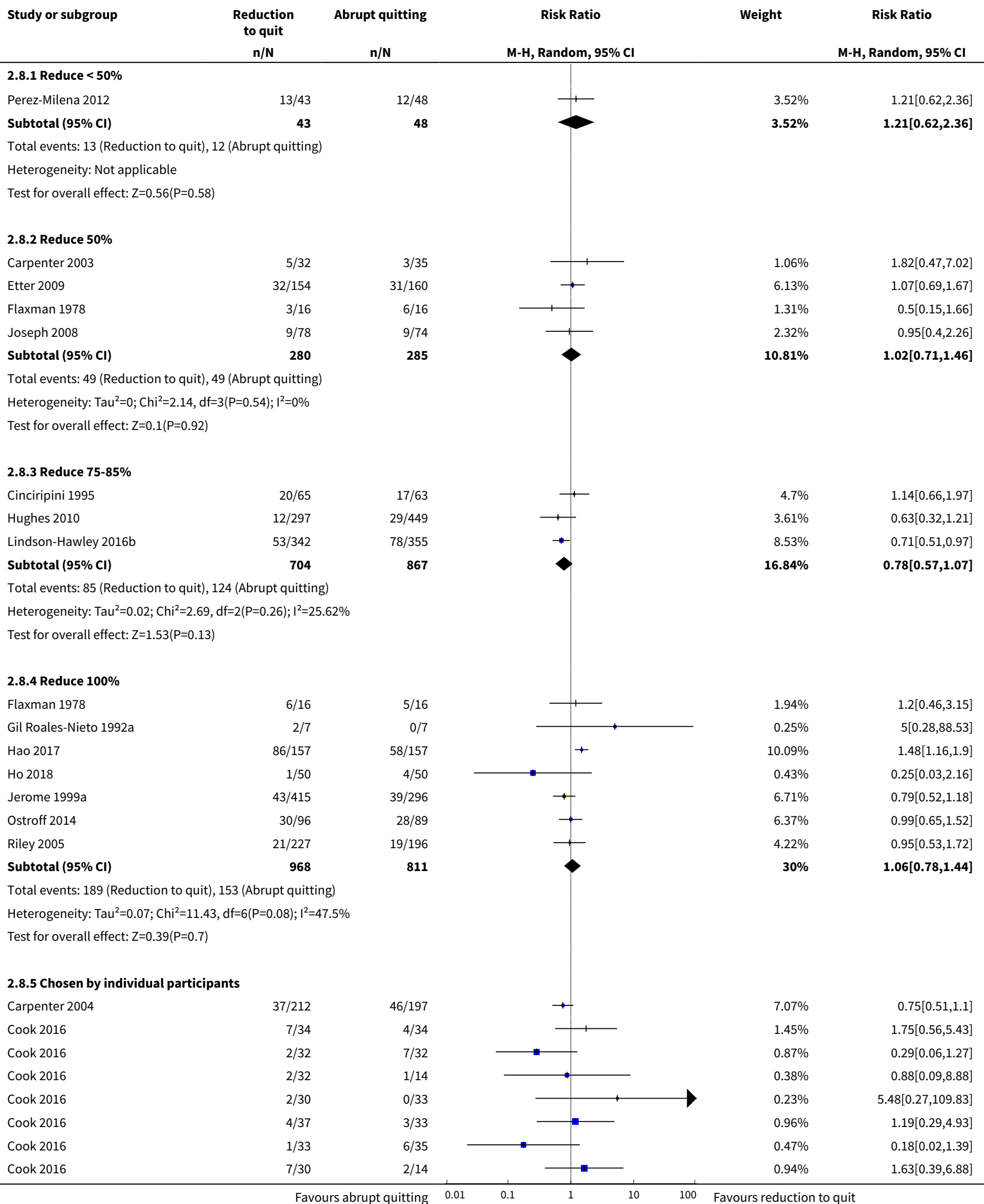




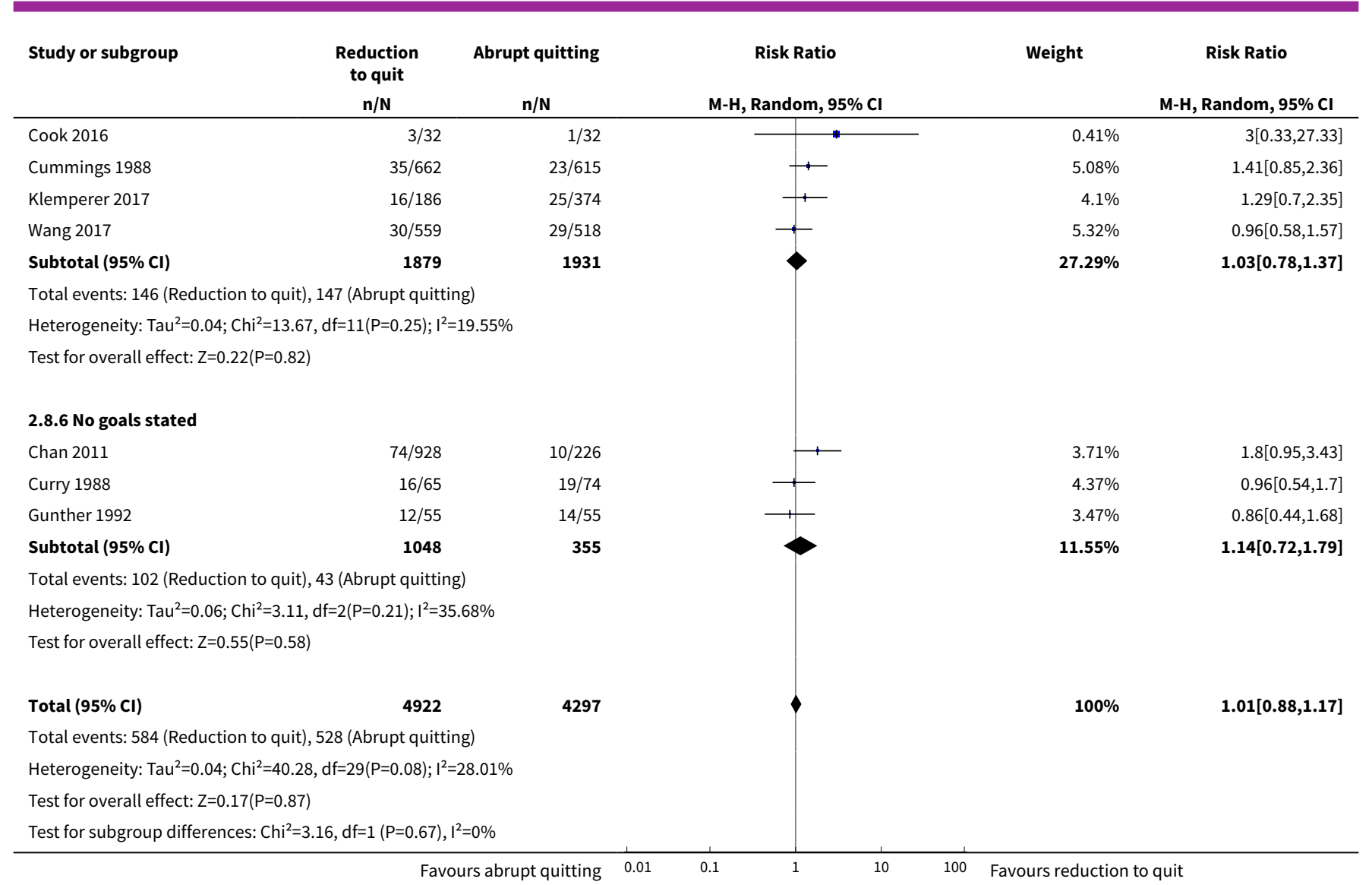

Analysis 2.9. Comparison 2 Reduction to quit versus abrupt quitting, Outcome 9 Quit attempts.

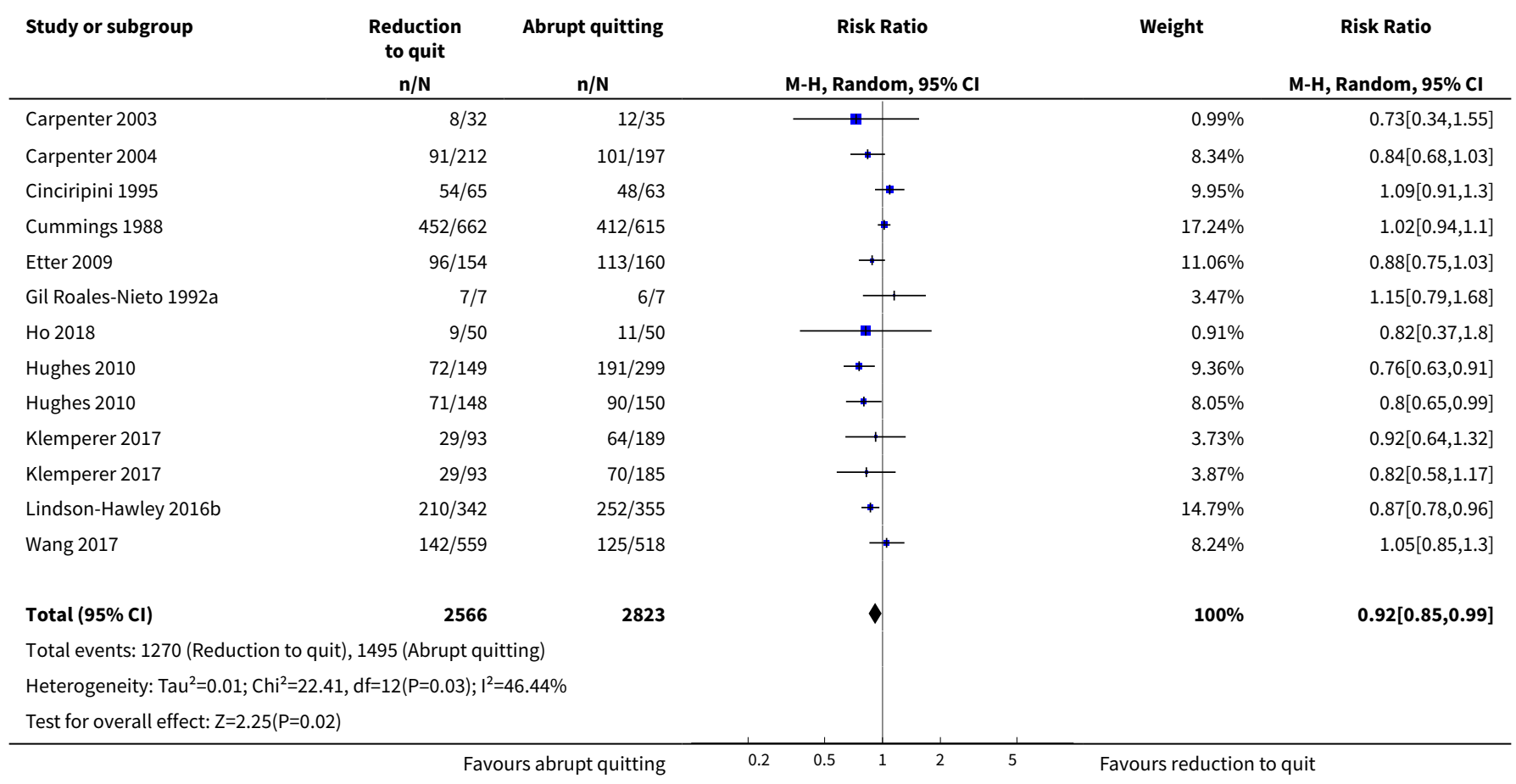




\section{Analysis 2.10. Comparison 2 Reduction to quit versus abrupt quitting, Outcome 10 Pre-quit reduction of at least $50 \%$.}

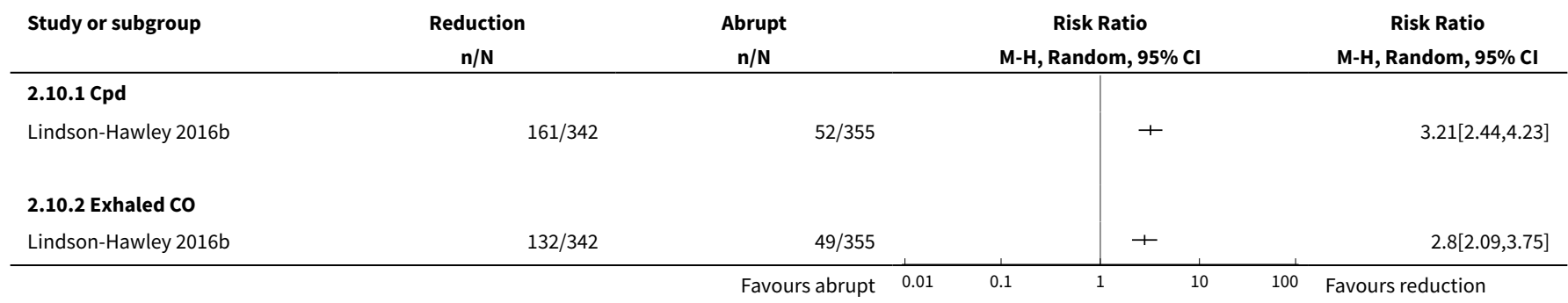

Analysis 2.11. Comparison 2 Reduction to quit versus abrupt quitting, Outcome 11 Pre-quit reduction in cpd.

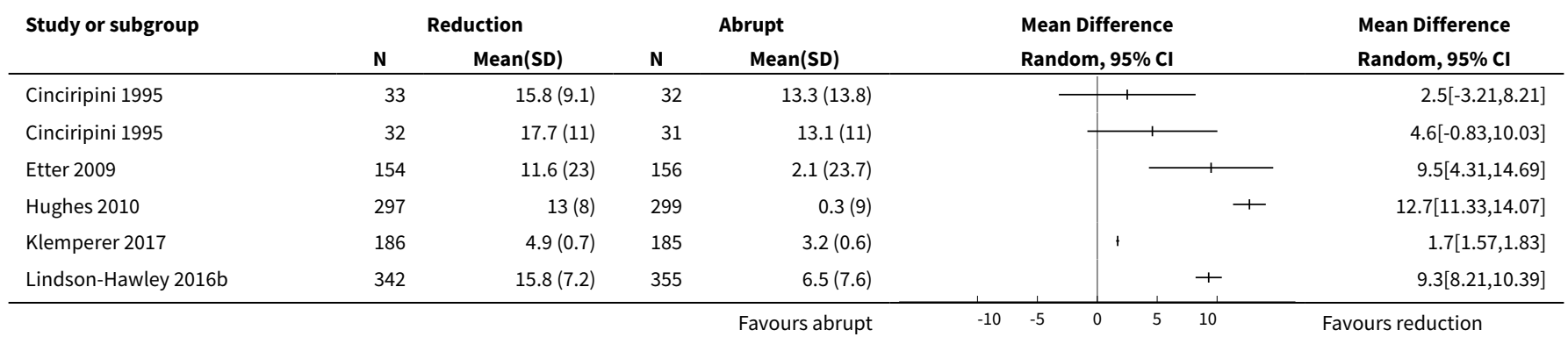

Analysis 2.12. Comparison 2 Reduction to quit versus abrupt quitting, Outcome 12 Pre-quit reduction in carbon monoxide.

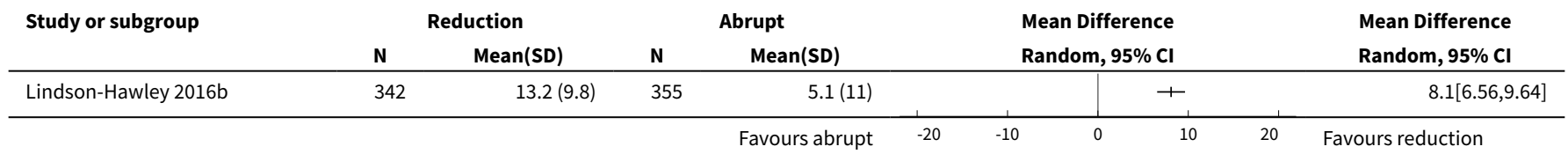

Analysis 2.13. Comparison 2 Reduction to quit versus abrupt quitting, Outcome 13 Pre-quit reduction in cotinine.

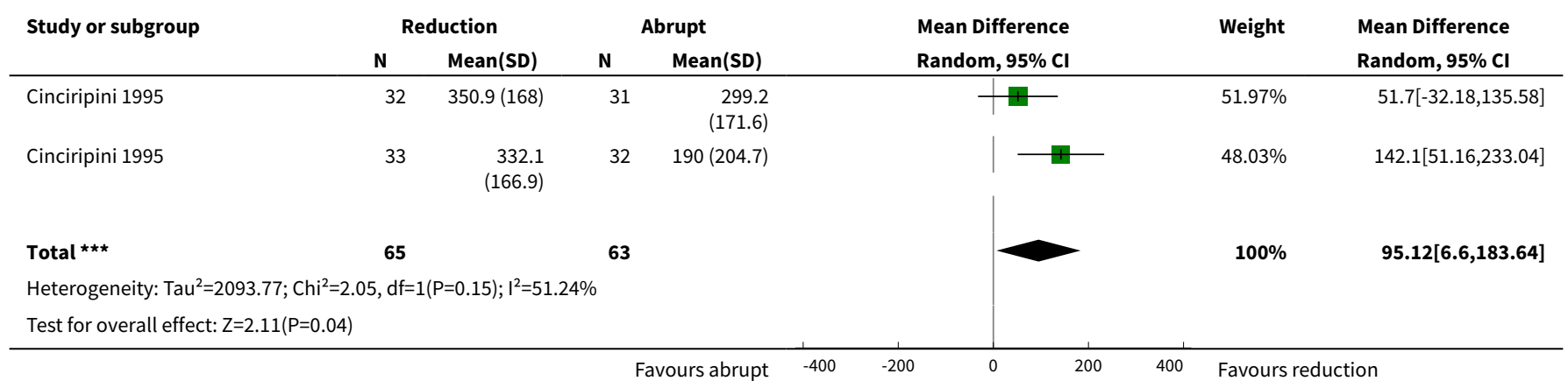


Analysis 2.14. Comparison 2 Reduction to quit versus abrupt quitting, Outcome 14 Pre-quit SAEs.

\begin{tabular}{|c|c|c|c|c|c|}
\hline Study or subgroup & $\begin{array}{c}\text { Reduction } \\
\mathrm{n} / \mathrm{N}\end{array}$ & $\begin{array}{c}\text { Abrupt } \\
\mathrm{n} / \mathrm{N}\end{array}$ & $\begin{array}{c}\text { Risk Ratio } \\
\text { M-H, Random, } 95 \% \mathrm{CI} \\
\end{array}$ & Weight & $\begin{array}{c}\text { Risk Ratio } \\
\text { M-H, Random, } 95 \% \mathrm{Cl} \\
\end{array}$ \\
\hline \multicolumn{6}{|l|}{ 2.14.1 NRT } \\
\hline Cook 2016 & $0 / 198$ & 0/198 & & & Not estimable \\
\hline Etter 2009 & $0 / 154$ & $0 / 160$ & & & Not estimable \\
\hline Joseph 2008 & $15 / 78$ & $13 / 74$ & - & $91.88 \%$ & $1.09[0.56,2.14]$ \\
\hline Lindson-Hawley 2016b & $3 / 342$ & $1 / 355$ & *- & $8.12 \%$ & $3.11[0.33,29.79]$ \\
\hline Subtotal $(95 \% \mathrm{Cl})$ & 772 & 787 & & $100 \%$ & $1.19[0.63,2.27]$ \\
\hline \multicolumn{6}{|c|}{ Total events: 18 (Reduction), 14 (Abrupt) } \\
\hline \multicolumn{6}{|c|}{ Heterogeneity: Tau $^{2}=0 ; \mathrm{Chi}^{2}=0.77, \mathrm{df}=1(\mathrm{P}=0.38) ; \mathrm{I}^{2}=0 \%$} \\
\hline \multicolumn{6}{|c|}{ Test for overall effect: $Z=0.53(P=0.59)$} \\
\hline \multicolumn{6}{|c|}{ 2.14.2 No pharmacotherapy } \\
\hline Cook 2016 & $0 / 62$ & $0 / 28$ & & & Not estimable \\
\hline Ho 2018 & $0 / 50$ & $0 / 50$ & & & Not estimable \\
\hline Klemperer 2017 & $0 / 186$ & $0 / 374$ & & & Not estimable \\
\hline Subtotal $(95 \% \mathrm{Cl})$ & 298 & 452 & & & Not estimable \\
\hline \multicolumn{6}{|c|}{ Total events: 0 (Reduction), 0 (Abrupt) } \\
\hline \multicolumn{6}{|c|}{ Heterogeneity: Not applicable } \\
\hline \multicolumn{6}{|c|}{ Test for overall effect: Not applicable } \\
\hline
\end{tabular}

Analysis 2.15. Comparison 2 Reduction to quit versus abrupt quitting, Outcome 15 Pre-quit tobacco withdrawal \& additional AE information.

\begin{tabular}{|c|c|c|c|}
\hline \multicolumn{4}{|c|}{ Pre-quit tobacco withdrawal \& additional AE information } \\
\hline Study & Withdrawal measurement method & Withdrawal measure findings & $\begin{array}{l}\text { Additional narrative } \\
\text { information on AEs }\end{array}$ \\
\hline Cinciripini 1995 & $\begin{array}{l}\text { "Hughes (Minnesota) Withdrawal } \\
\text { Symptoms Checklist (Hughes 1986), } \\
\text { which provided a total score using rat- } \\
\text { ings from } 0 \text { to } 3 \text { of } 17 \text { symptoms of to- } \\
\text { bacco withdrawal (e.g., irritability, } \\
\text { hunger, sleep disturbance, etc.)" N.B. } \\
\text { this description of the Minnesota Nico- } \\
\text { tine Withdrawal Scale does not appear } \\
\text { to be accurate - unclear whether inves- } \\
\text { tigators implemented in this way or as } \\
\text { intended by the authors }\end{array}$ & $\begin{array}{l}\text { Scheduled reduction group: baseline } \\
10.6 \text {; quit day } 15.8 \text { (change }+5.2 \text { ) } \\
\text { Non-scheduled reduction group: base- } \\
\text { line } 11.2 \text {; quit day } 19.2 \text { (change }+8.0 \text { ) } \\
\text { Scheduled abrupt group: baseline } 11.9 ; \\
\text { quit day } 18.1 \text { (change }+6.2 \text { ) } \\
\text { Non-scheduled abrupt group: baseline } \\
10.1 \text {; quit day } 18.5 \text { (change }+8.4 \text { ) (esti- } \\
\text { mated from Figure } 1 \text { in main paper). } \\
\text { Withdrawal symptoms therefore went } \\
\text { up in all study arms and were similar } \\
\text { between the reduction and abrupt quit- } \\
\text { ting groups. }\end{array}$ & $\mathrm{n} / \mathrm{a}$ \\
\hline Etter 2009 & $\begin{array}{l}\text { Minnesota Withdrawal Form (Hughes } \\
\text { 1986) and Cigarette Withdrawal Scale } \\
\text { (Etter 2005) }\end{array}$ & $\begin{array}{l}\text { In both groups, levels of craving among } \\
\text { quitters decreased significantly and } \\
\text { levels of appetite and hunger increased } \\
\text { significantly between baseline and the } \\
\text { 3-day survey. In the usual-care 'abrupt' } \\
\text { group only, a statistically significant } \\
\text { increase was noted between baseline } \\
\text { and the 3-day survey in ratings on the } \\
\text { Minnesota Withdrawal Form and in anx- } \\
\text { iety and depression. However, the dif- } \\
\text { ferences between groups in change of } \\
\text { withdrawal symptoms over time were } \\
\text { not statistically significant. }\end{array}$ & $\mathrm{n} / \mathrm{a}$ \\
\hline Hughes 2010 & Craving measured on a scale from 1 to 5 & $\begin{array}{l}\text { Craving decreased in the gradual 're- } \\
\text { duction' condition ( } 4.5 \text { to } 4.0 \text { ) from } \\
\text { baseline to quit day, but did not do } \\
\text { so in the 'abrupt' conditions ( } 4.5 \text { to }\end{array}$ & $\mathrm{n} / \mathrm{a}$ \\
\hline
\end{tabular}




\begin{tabular}{|c|c|c|c|}
\hline \multicolumn{4}{|c|}{ Pre-quit tobacco withdrawal \& additional AE information } \\
\hline Study & Withdrawal measurement method & Withdrawal measure findings & $\begin{array}{l}\text { Additional narrative } \\
\text { information on AEs }\end{array}$ \\
\hline & & $\begin{array}{l}4.4, F(2,508)=9.16, P<0.001) \text { resulting } \\
\text { in a between-group difference. }\end{array}$ & \\
\hline Lindson-Hawley $2016 \mathrm{~b}$ & $\mathrm{n} / \mathrm{a}$ & $\mathrm{n} / \mathrm{a}$ & $\begin{array}{l}\text { "Most symptoms of nicotine overdose } \\
\text { were uncommon and mild and did not } \\
\text { differ by group. Salivating ( } 2 \text { nd pre-quit } \\
\text { week, reduction: } 18 / 120(15 \%) \text {; abrupt: } \\
17 / 259(6.6 \%) \\
\text { and cold sweats ( } 2 \text { nd pre-quit week, re- } \\
\text { duction: } 15 / 121(12.4 \%) \text {; abrupt: } 11 / 261 \\
(4.2 \%)) \text { were more common in the re- } \\
\text { duction group than abrupt group in } \\
\text { both pre-quit weeks" }\end{array}$ \\
\hline
\end{tabular}

\section{Analysis 2.16. Comparison 2 Reduction to quit versus abrupt quitting, Outcome 16 Moderation of the reduction vs abrupt quitting effect.}

\begin{tabular}{|c|c|c|}
\hline \multicolumn{3}{|c|}{ Moderation of the reduction vs abrupt quitting effect } \\
\hline Study & Potential moderator(s) assessed & Narrative findings \\
\hline Curry 1988 & Self-efficacy & $\begin{array}{l}\text { Self-efficacy did not moderate the relationship be- } \\
\text { tween trial arm and long-term quitting. }\end{array}$ \\
\hline Hughes 2010 & $\begin{array}{l}\text { Motivation to quit; self-efficacy; preference for reduc- } \\
\text { tion to quit versus abrupt quitting }\end{array}$ & $\begin{array}{l}\text { Among smokers with high self-efficacy, the abrupt } \\
\text { condition out-performed the gradual condition, but } \\
\text { among smokers with low self-efficacy, the abrupt con- } \\
\text { dition did not out-perform the gradual condition ( } P \\
=0.03 \text { ). A similar, but non-significant, interaction oc- } \\
\text { curred with self-rated confidence in quitting (self-effi- } \\
\text { cacy). Relative preference for gradual vs abrupt cessa- } \\
\text { tion did not predict response to treatment. }\end{array}$ \\
\hline Lindson-Hawley 2016b & Preference for reduction to quit versus abrupt quitting & $\begin{array}{l}\text { "Participants who preferred gradual cessation were } \\
\text { significantly less likely to be abstinent at } 4 \text { weeks } \\
\text { than those who preferred abrupt ( } 38.3 \% \text { vs } 52.2 \% \text {; P } \\
=0.007) \text {. Among those who preferred gradual cessa- } \\
\text { tion and were allocated to quit abruptly against their } \\
\text { preference, abstinence at } 4 \text { weeks was } 42.0 \% \text { com- } \\
\text { pared with } 34.6 \% \text { among those assigned to gradual } \\
\text { cessation (not statistically different; } \mathrm{P}=0.152) \text {. The } \\
\text { RRs for achieving abstinence for the gradual-cessa- } \\
\text { tion group compared with the abrupt-cessation group, } \\
\text { stratified by baseline preference, were as follows: pre- } \\
\text { fer gradual cessation, } 0.82(\mathrm{Cl}, 0.64 \text { to } 1.07) \text {; no prefer- } \\
\text { ence, } 0.80(\mathrm{Cl}, 0.49 \text { to } 1.07) \text {; and prefer abrupt cessa- } \\
\text { tion, } 0.79(\mathrm{Cl}, 0.60 \text { to } 1.08) \text { ". RRs were therefore similar } \\
\text { across preferences. }\end{array}$ \\
\hline
\end{tabular}

\section{Comparison 3. Reduction + pharmacotherapy versus reduction alone}

\begin{tabular}{llllll}
\hline Outcome or subgroup title & $\begin{array}{l}\text { No. of } \\
\text { studies }\end{array}$ & $\begin{array}{l}\text { No. of } \\
\text { partici- } \\
\text { pants }\end{array}$ & Statistical method & Effect size \\
\hline 1 Abstinence & 11 & 8636 & Risk Ratio (M-H, Random, 95\% Cl) & $1.68[1.09,2.58]$ \\
\hline 1.1 Combination NRT & 3 & 1124 & Risk Ratio (M-H, Random, 95\% Cl) & $1.02[0.61,1.69]$ \\
\hline 1.2 Nicotine patch & 1 & 85 & Risk Ratio (M-H, Random, 95\% Cl) & $0.34[0.02,5.31]$ \\
\hline 1.3 Fast acting NRT & 7 & 5323 & Risk Ratio (M-H, Random, 95\% Cl) & $2.56[1.93,3.39]$ \\
\hline 1.4 Varenicline & 1 & 1510 & Risk Ratio (M-H, Random, 95\% Cl) & $3.99[2.93,5.44]$ \\
\hline
\end{tabular}




\begin{tabular}{|c|c|c|c|c|}
\hline Outcome or subgroup title & $\begin{array}{l}\text { No. of } \\
\text { studies }\end{array}$ & $\begin{array}{l}\text { No. of } \\
\text { partici- } \\
\text { pants }\end{array}$ & Statistical method & Effect size \\
\hline 1.5 Bupropion & 1 & 594 & Risk Ratio (M-H, Random, 95\% Cl) & $1.27[0.67,2.40]$ \\
\hline 2 Quit attempts & 1 & & Risk Ratio (M-H, Random, 95\% Cl) & Totals not selected \\
\hline 2.1 Combination NRT & 1 & & Risk Ratio (M-H, Random, 95\% Cl) & $0.0[0.0,0.0]$ \\
\hline $\begin{array}{l}3 \text { Pre-quit cpd reduction of } \geq 50 \% \text { or } \\
75 \%\end{array}$ & 7 & 3472 & Risk Ratio (M-H, Random, 95\% Cl) & $1.60[1.22,2.10]$ \\
\hline 3.1 Combination NRT & 1 & 534 & Risk Ratio (M-H, Random, 95\% Cl) & $1.26[0.98,1.62]$ \\
\hline 3.2 Fast acting NRT & 5 & 1428 & Risk Ratio (M-H, Random, 95\% Cl) & $1.75[1.08,2.83]$ \\
\hline 3.3 Varenicline & 1 & 1510 & Risk Ratio (M-H, Random, 95\% Cl) & $1.75[1.42,2.15]$ \\
\hline 4 Pre-quit reduction in cpd & 4 & & $\begin{array}{l}\text { Mean Difference (IV, Random, 95\% } \\
\mathrm{CI} \text { ) }\end{array}$ & Subtotals only \\
\hline 4.1 Combination NRT & 1 & 534 & $\begin{array}{l}\text { Mean Difference (IV, Random, 95\% } \\
\mathrm{CI})\end{array}$ & $2.20[0.40,4.00]$ \\
\hline 4.2 Nicotine patch & 1 & 70 & $\begin{array}{l}\text { Mean Difference (IV, Random, 95\% } \\
\mathrm{CI})\end{array}$ & $-0.10[-2.63,2.43]$ \\
\hline 4.3 Fast-acting NRT & 3 & 898 & $\begin{array}{l}\text { Mean Difference (IV, Random, 95\% } \\
\mathrm{CI} \text { ) }\end{array}$ & $1.16[-0.09,2.41]$ \\
\hline $\begin{array}{l}5 \text { Pre-quit reduction in carbon } \\
\text { monoxide }\end{array}$ & 2 & & $\begin{array}{l}\text { Mean Difference (IV, Random, 95\% } \\
\mathrm{Cl} \text { ) }\end{array}$ & Totals not selected \\
\hline 5.1 Nicotine patch & 1 & & $\begin{array}{l}\text { Mean Difference (IV, Random, 95\% } \\
\mathrm{CI} \text { ) }\end{array}$ & $0.0[0.0,0.0]$ \\
\hline 5.2 Fast acting NRT & 2 & & $\begin{array}{l}\text { Mean Difference (IV, Random, 95\% } \\
\mathrm{CI} \text { ) }\end{array}$ & $0.0[0.0,0.0]$ \\
\hline 6 Pre-quit AEs & 2 & & Risk Ratio (M-H, Random, 95\% Cl) & Totals not selected \\
\hline 7 Pre-quit SAEs & 2 & 762 & Risk Ratio (M-H, Random, 95\% Cl) & $7.28[0.38,140.28]$ \\
\hline $\begin{array}{l}8 \text { Pre-quit tobacco withdrawal \& ad- } \\
\text { ditional AE information }\end{array}$ & & & Other data & No numeric data \\
\hline
\end{tabular}

Analysis 3.1. Comparison 3 Reduction + pharmacotherapy versus reduction alone, Outcome 1 Abstinence.

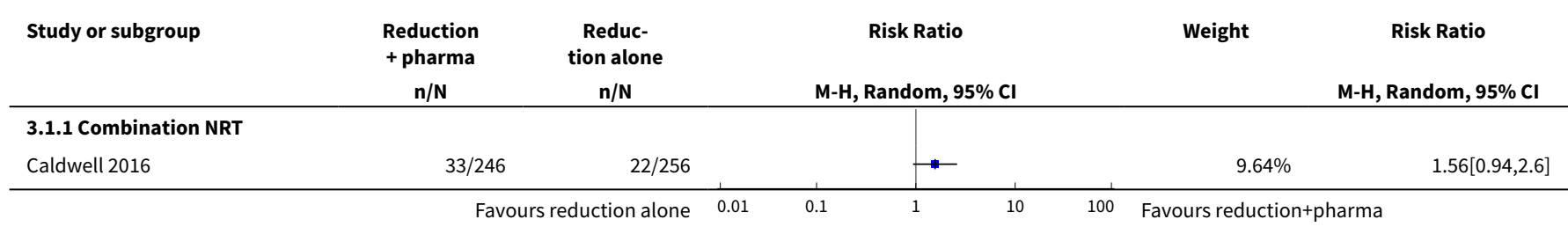




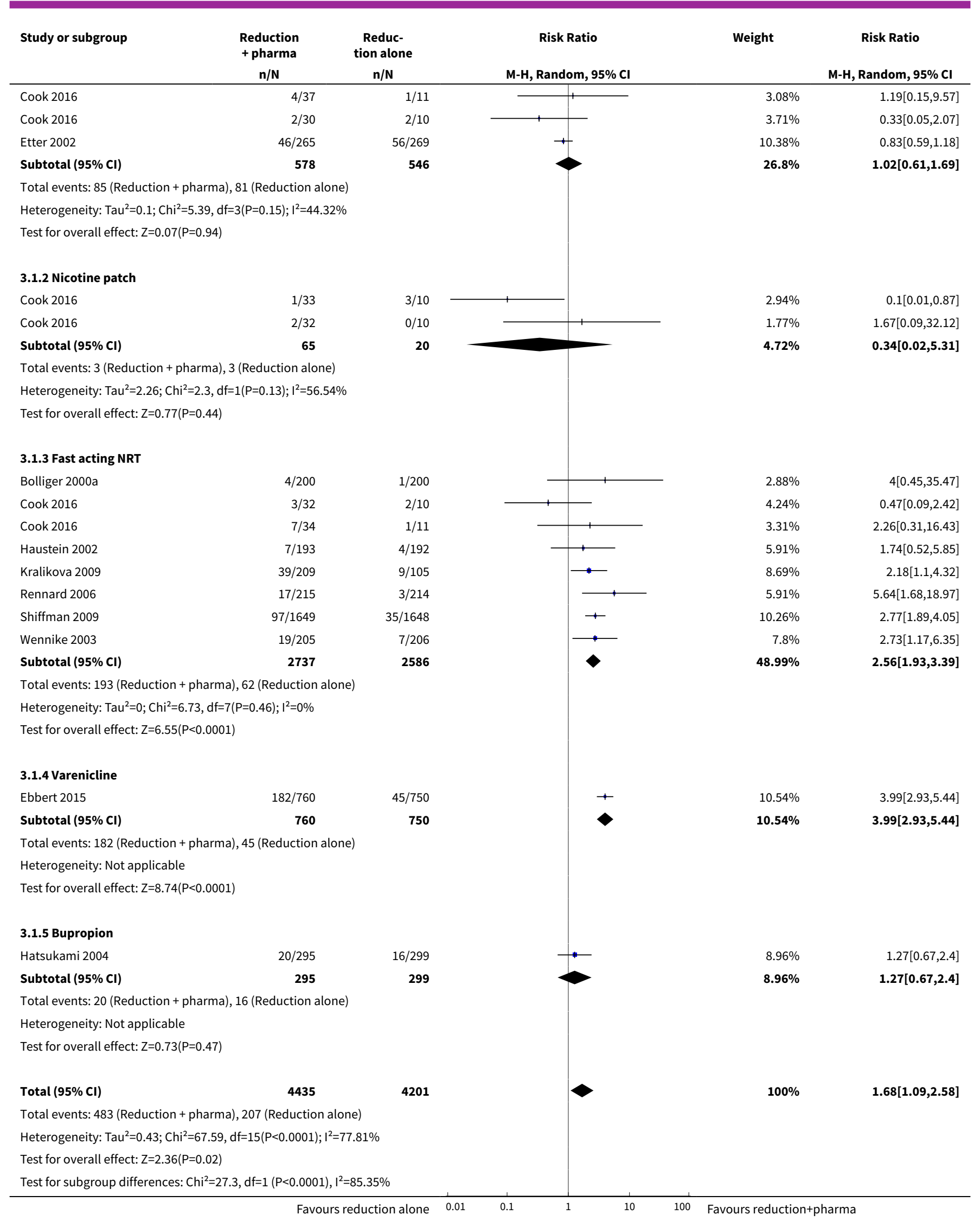


Analysis 3.2. Comparison 3 Reduction + pharmacotherapy versus reduction alone, Outcome 2 Quit attempts.

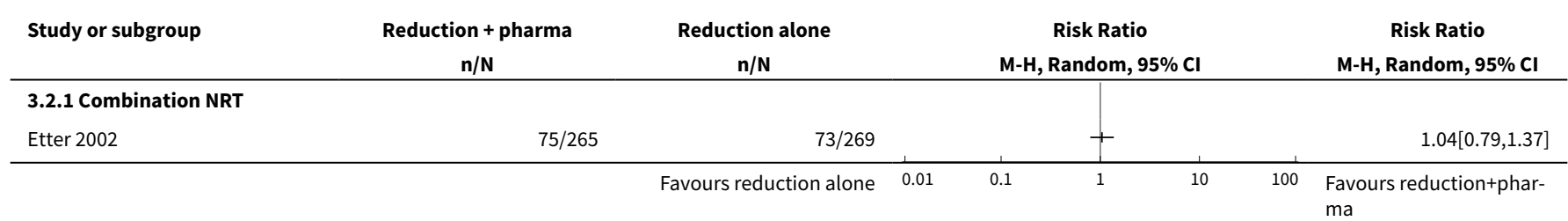

Analysis 3.3. Comparison 3 Reduction + pharmacotherapy versus reduction alone, Outcome 3 Pre-quit cpd reduction of $\geq 50 \%$ or $75 \%$.

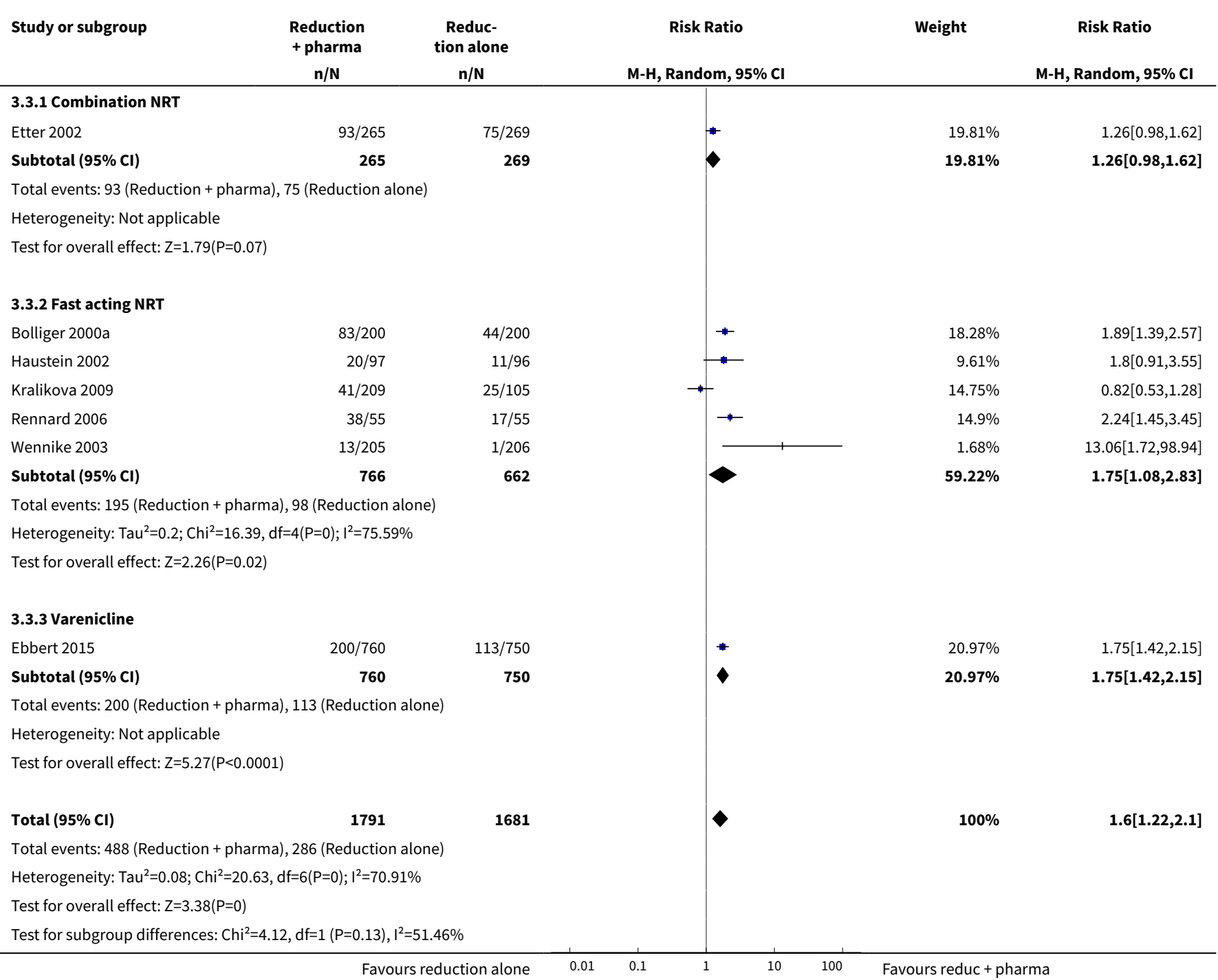


Analysis 3.4. Comparison 3 Reduction + pharmacotherapy versus reduction alone, Outcome 4 Pre-quit reduction in cpd.

\begin{tabular}{|c|c|c|c|c|c|c|c|}
\hline \multirow[t]{2}{*}{ Study or subgroup } & \multicolumn{2}{|c|}{ Reduction + pharma } & \multicolumn{2}{|c|}{ Reduction alone } & \multirow{2}{*}{$\begin{array}{l}\text { Mean Difference } \\
\text { Random, } 95 \% \mathrm{Cl}\end{array}$} & \multirow[t]{2}{*}{ Weight } & \multirow{2}{*}{$\begin{array}{l}\text { Mean Difference } \\
\text { Random, } 95 \% \mathrm{Cl}\end{array}$} \\
\hline & $\mathbf{N}$ & $\operatorname{Mean}(S D)$ & $\mathbf{N}$ & Mean(SD) & & & \\
\hline \multicolumn{8}{|c|}{ 3.4.1 Combination NRT } \\
\hline Etter 2002 & 265 & $10.9(11.2)$ & 269 & $8.7(10)$ & & $100 \%$ & $2.2[0.4,4]$ \\
\hline 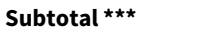 & 265 & & 269 & & & $100 \%$ & $2.2[0.4,4]$ \\
\hline \multicolumn{8}{|c|}{ Heterogeneity: Not applicable } \\
\hline \multicolumn{8}{|c|}{ Test for overall effect: $\mathrm{Z}=2.39(\mathrm{P}=0.02)$} \\
\hline \multicolumn{8}{|l|}{ 3.4.2 Nicotine patch } \\
\hline Hanson 2008 & 34 & $6.1(4.9)$ & 36 & $6.2(5.9)$ & & $100 \%$ & $-0.1[-2.63,2.43]$ \\
\hline Subtotal $\star \star \star$ & 34 & & 36 & & & $100 \%$ & $-0.1[-2.63,2.43]$ \\
\hline \multicolumn{8}{|c|}{ Heterogeneity: Not applicable } \\
\hline \multicolumn{8}{|c|}{ Test for overall effect: $\mathrm{Z}=0.08(\mathrm{P}=0.94)$} \\
\hline Bolliger 2000a & 200 & $16.2(11.4)$ & 200 & $14.5(12.1)$ & & $29.41 \%$ & $1.7[-0.6,4]$ \\
\hline Hanson 2008 & 33 & $6.7(4.2)$ & 36 & $6.2(5.9)$ & & $26.89 \%$ & $0.5[-1.91,2.91]$ \\
\hline Rennard 2006 & 215 & $14.6(8.6)$ & 214 & $13.4(11.2)$ & +1 & $43.7 \%$ & $1.2[-0.69,3.09]$ \\
\hline Subtotal $\star \star \star$ & 448 & & 450 & & & $100 \%$ & $1.16[-0.09,2.41]$ \\
\hline \multicolumn{8}{|c|}{ Heterogeneity: $\mathrm{Tau}^{2}=0 ; \mathrm{Chi}^{2}=0.5, \mathrm{df}=2(\mathrm{P}=0.78) ; \mathrm{I}^{2}=0 \%$} \\
\hline \multicolumn{8}{|c|}{ Test for overall effect: $\mathrm{Z}=1.82(\mathrm{P}=0.07)$} \\
\hline \multicolumn{8}{|c|}{ Test for subgroup differences: $\mathrm{Chi}^{2}=2.18, \mathrm{df}=1(\mathrm{P}=0.34), \mathrm{I}^{2}=8.15 \%$} \\
\hline & & & urs re & ction alone & -2 & Favours & ion+pharma \\
\hline
\end{tabular}

Analysis 3.5. Comparison 3 Reduction + pharmacotherapy versus reduction alone, Outcome 5 Pre-quit reduction in carbon monoxide.

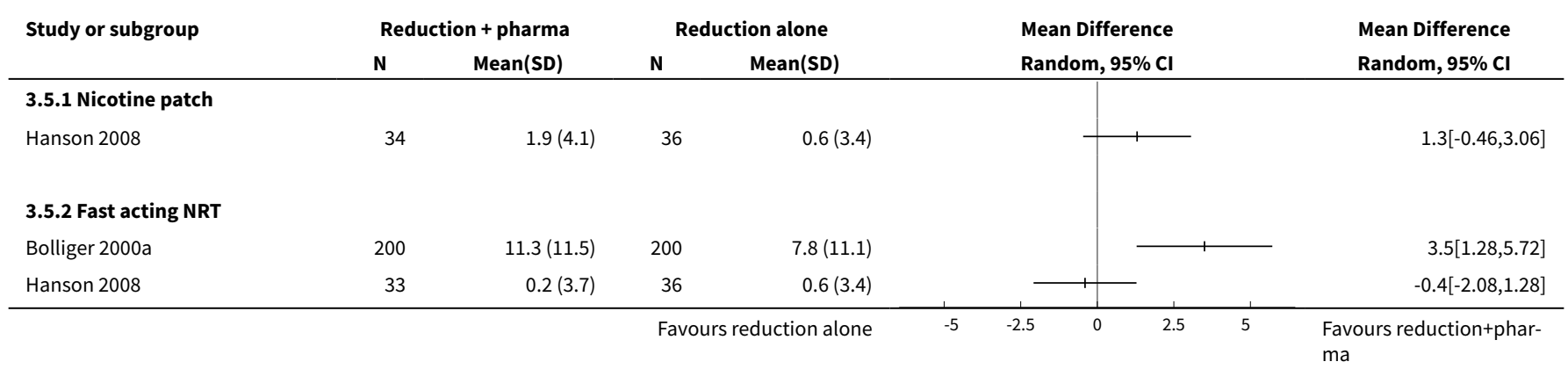

\section{Analysis 3.6. Comparison 3 Reduction + pharmacotherapy versus reduction alone, Outcome 6 Pre-quit AEs.}

\begin{tabular}{|c|c|c|c|c|}
\hline Study or subgroup & $\begin{array}{c}\text { Reduction + pharma } \\
n / N\end{array}$ & $\begin{array}{c}\text { Reduction alone } \\
\mathrm{n} / \mathrm{N}\end{array}$ & $\begin{array}{c}\text { Risk Ratio } \\
\text { M-H, Random, } 95 \% \mathrm{CI}\end{array}$ & $\begin{array}{c}\text { Risk Ratio } \\
\text { M-H, Random, 95\% Cl }\end{array}$ \\
\hline Bolliger 2000a & $113 / 200$ & $114 / 200$ & $\longrightarrow$ & $0.99[0.84,1.18]$ \\
\hline Shiffman 2009 & $795 / 1649$ & $603 / 1648$ & & $1.32[1.22,1.43]$ \\
\hline
\end{tabular}


Analysis 3.7. Comparison 3 Reduction + pharmacotherapy versus reduction alone, Outcome 7 Pre-quit SAEs.

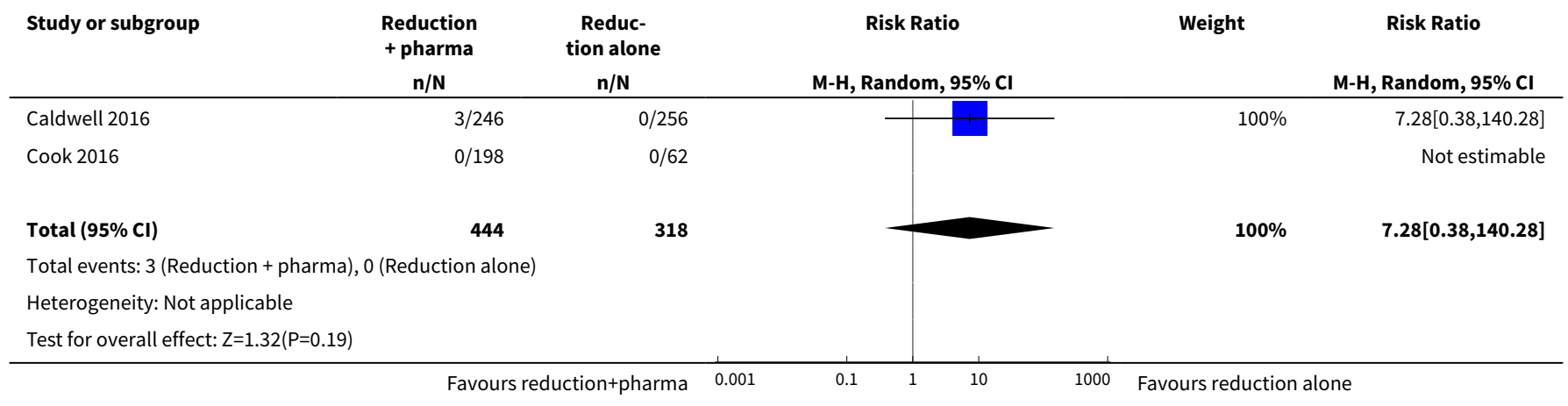

Analysis 3.8. Comparison 3 Reduction + pharmacotherapy versus reduction alone, Outcome 8 Pre-quit tobacco withdrawal \& additional AE information.

\begin{tabular}{|c|c|c|}
\hline \multirow[b]{2}{*}{ Study } & \multicolumn{2}{|c|}{ Pre-quit tobacco withdrawal \& additional AE information } \\
\hline & Withdrawal measurement method & Withdrawal measure findings \\
\hline Bolliger 2000a & $\mathrm{n} / \mathrm{a}$ & $\mathrm{n} / \mathrm{a}$ \\
\hline Caldwell 2016 & $\begin{array}{l}\text { Minnesota nicotine withdrawal scale } \\
\text { (Hughes 1986) }\end{array}$ & $\begin{array}{l}\text { The two groups had similar nicotine } \\
\text { withdrawal scores up to one day post- } \\
\text { quit (no significant difference) }\end{array}$ \\
\hline
\end{tabular}

\begin{tabular}{|c|c|c|}
\hline Etter 2002 & $\mathrm{n} / \mathrm{a}$ & $\mathrm{n} / \mathrm{a}$ \\
\hline Haustein 2002 & $\begin{array}{l}\text { Minnesota nicotine withdrawal scale } \\
\text { (Hughes 1986) }\end{array}$ & $\begin{array}{l}\text { Both NRT groups and the short-term } \\
\text { placebo group experienced statisti- } \\
\text { cally significant decreases in urge to } \\
\text { smoke. There was a statistically signif- } \\
\text { icant decrease in restlessness in the } \\
\text { shorter active treatment group, and in } \\
\text { difficulty concentrating in the longer } \\
\text { placebo group. There were no signif- } \\
\text { icant within-group changes in any of } \\
\text { the other symptoms (anxiety, increased } \\
\text { appetite, insomnia, irritability/frustra- } \\
\text { tion) between baseline and month } 4 \text {. } \\
\text { Between-group significance testing was } \\
\text { not reported. }\end{array}$ \\
\hline Shiffman 2009 & $\mathrm{n} / \mathrm{a}$ & $\mathrm{n} / \mathrm{a}$ \\
\hline
\end{tabular}


Comparison 4. Modality of reduction support

\begin{tabular}{llllll}
\hline Outcome or subgroup title & $\begin{array}{l}\text { No. of } \\
\text { studies }\end{array}$ & $\begin{array}{l}\text { No. of par- } \\
\text { ticipants }\end{array}$ & Statistical method & Effect size \\
\hline 1 Abstinence & 4 & 296 & Risk Ratio (M-H, Random, 95\% Cl) & $1.99[1.21,3.27]$ \\
\hline 2 Pre-quit reduction in cpd & 2 & 107 & Mean Difference (IV, Random, 95\% Cl) & $7.00[3.50,10.50]$ \\
\hline
\end{tabular}

Analysis 4.1. Comparison 4 Modality of reduction support, Outcome 1 Abstinence.

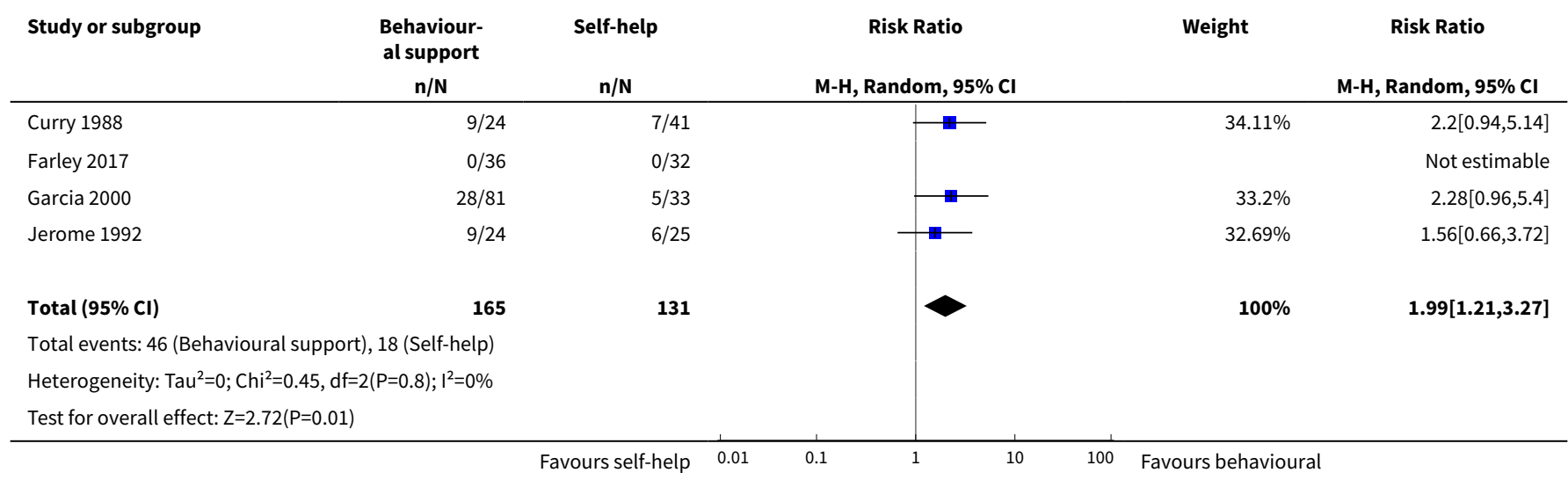

Analysis 4.2. Comparison 4 Modality of reduction support, Outcome 2 Pre-quit reduction in cpd.

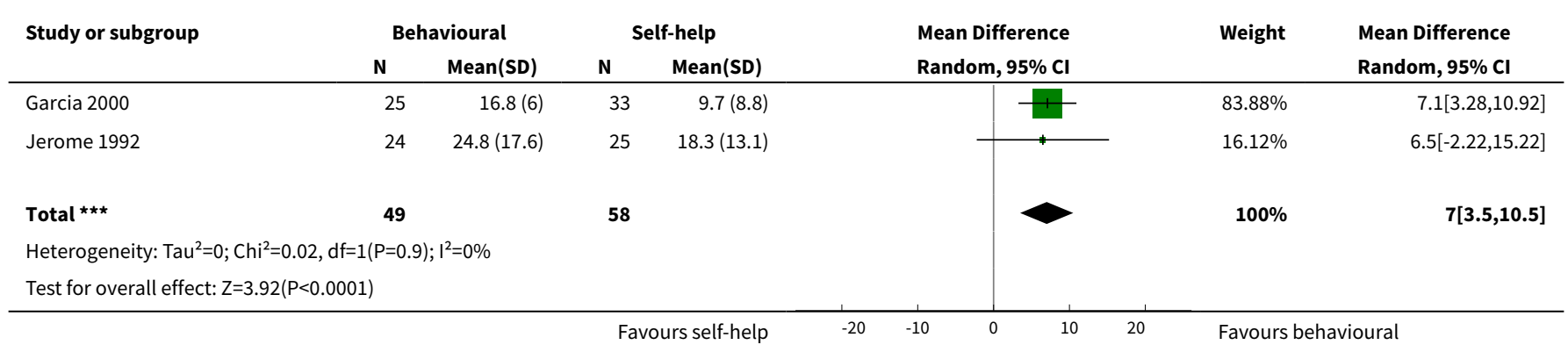

\section{Comparison 5. Length of reduction}

\begin{tabular}{lllll}
\hline Outcome or subgroup title & $\begin{array}{l}\text { No. of } \\
\text { studies }\end{array}$ & $\begin{array}{l}\text { No. of } \\
\text { partici- } \\
\text { pants }\end{array}$ & Statistical method & Effect size \\
\hline 1 Abstinence & 2 & 453 & Risk Ratio $(\mathrm{M}-\mathrm{H}$, Random, $95 \% \mathrm{Cl})$ & $0.22[0.05,1.01]$ \\
\hline 2 Pre-quit cpd reduction $\geq 50 \%$ & 1 & Risk Ratio $(\mathrm{M}-\mathrm{H}$, Random, $95 \% \mathrm{Cl})$ & Totals not selected \\
\hline 3 Pre-quit tobacco withdrawal & & Other data & No numeric data \\
\hline
\end{tabular}


Analysis 5.1. Comparison 5 Length of reduction, Outcome 1 Abstinence.

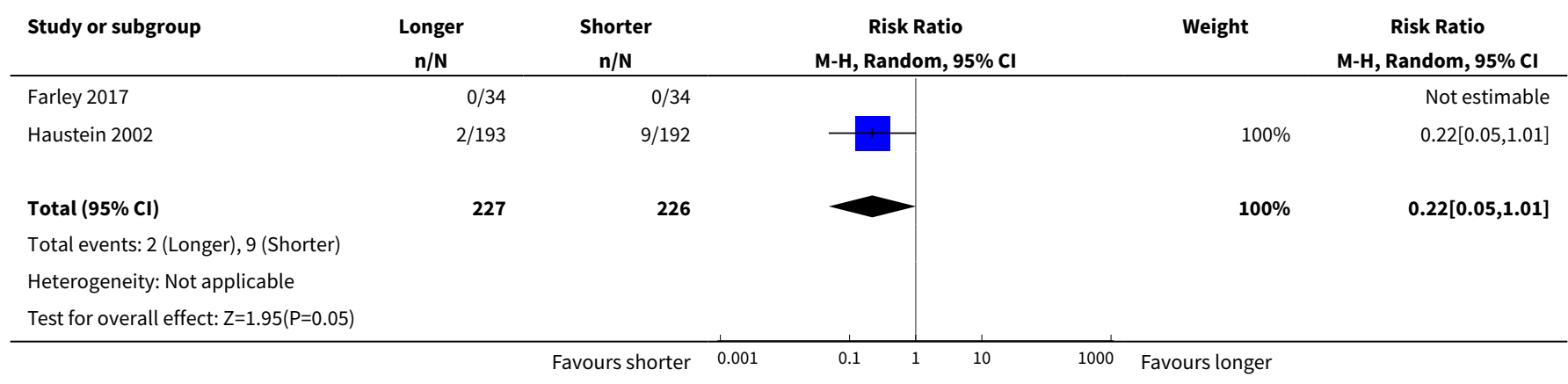

Analysis 5.2. Comparison 5 Length of reduction, Outcome 2 Pre-quit cpd reduction $\geq 50 \%$.

\begin{tabular}{|c|c|c|c|c|c|c|}
\hline \multirow{2}{*}{$\begin{array}{l}\text { Study or subgroup } \\
\text { Haustein } 2002\end{array}$} & $\begin{array}{c}\text { Longer } \\
\mathbf{n} / \mathbf{N}\end{array}$ & $\begin{array}{c}\text { Shorter } \\
\mathrm{n} / \mathrm{N} \\
\end{array}$ & & \multicolumn{2}{|c|}{$\begin{array}{c}\text { Risk Ratio } \\
\text { M-H, Random, } 95 \% \text { Cl }\end{array}$} & \multirow{2}{*}{$\begin{array}{c}\text { Risk Ratio } \\
\text { M-H, Random, 95\% Cl } \\
0.58[0.39,0.86\end{array}$} \\
\hline & $31 / 193$ & $53 / 192$ & & + & & \\
\hline
\end{tabular}

Analysis 5.3. Comparison 5 Length of reduction, Outcome 3 Pre-quit tobacco withdrawal.

Pre-quit tobacco withdrawal

Study Withdrawal measurement method

Withdrawal measure findings

Both the longer and shorter reduction groups assigned to NRT and the shorter placebo group experienced statistically significant decreases in urge to smoke. There was a statistically significant decrease in restlessness in the shorter NRT group, and in difficulty concentrating in the longer placebo group.

There were no significant within-group changes in any of the other symptoms (anxiety, increased appetite, insomnia, irritability/frustration) between base-

line and month four. Between-group significance testing was not reported.

\section{Comparison 6. More structured vs less structured reduction}

\begin{tabular}{lllll}
\hline Outcome or subgroup title & $\begin{array}{l}\text { No. of } \\
\text { studies }\end{array}$ & $\begin{array}{l}\text { No. of } \\
\text { partici- } \\
\text { pants }\end{array}$ & Statistical method & Effect size \\
\hline 1 Abstinence & 2 & & Risk Ratio (M-H, Random, 95\% Cl) & Totals not selected \\
\hline 2 Quit attempts & 2 & 727 & Risk Ratio (M-H, Random, 95\% Cl) & $0.99[0.82,1.18]$ \\
\hline 3 Pre-quit reduction in cpd & 1 & & $\begin{array}{l}\text { Mean Difference (IV, Random, } \\
95 \% \text { Cl) }\end{array}$ & Totals not selected \\
\hline \hline
\end{tabular}




\begin{tabular}{llll}
\hline Outcome or subgroup title & $\begin{array}{l}\text { No. of } \\
\text { studies }\end{array}$ & $\begin{array}{l}\text { No. of } \\
\text { partici- } \\
\text { pants }\end{array}$ & Statistical method size \\
\hline
\end{tabular}

Analysis 6.1. Comparison 6 More structured vs less structured reduction, Outcome 1 Abstinence.

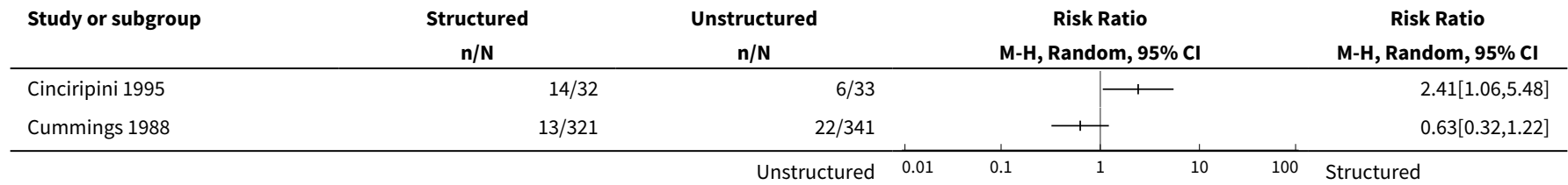

Analysis 6.2. Comparison 6 More structured vs less structured reduction, Outcome 2 Quit attempts.

\begin{tabular}{|c|c|c|c|c|c|}
\hline Study or subgroup & $\begin{array}{c}\text { Structured } \\
\mathrm{n} / \mathrm{N} \\
\end{array}$ & $\begin{array}{c}\text { Unstructured } \\
\mathrm{n} / \mathrm{N} \\
\end{array}$ & $\begin{array}{c}\text { Risk Ratio } \\
\text { M-H, Random, } 95 \% \text { Cl } \\
\end{array}$ & Weight & $\begin{array}{c}\text { Risk Ratio } \\
\text { M-H, Random, } 95 \% \text { CI } \\
\end{array}$ \\
\hline Cinciripini 1995 & $28 / 32$ & $26 / 33$ & 典 & $36.45 \%$ & $1.11[0.89,1.38]$ \\
\hline Cummings 1988 & $210 / 321$ & $242 / 341$ & & $63.55 \%$ & $0.92[0.83,1.02]$ \\
\hline Total $(95 \% \mathrm{Cl})$ & 353 & 374 & $\hat{\gamma}$ & $100 \%$ & $0.99[0.82,1.18]$ \\
\hline \multicolumn{6}{|c|}{ Total events: 238 (Structured), 268 (Unstructured) } \\
\hline Test for overall effect & & & & & \\
\hline
\end{tabular}

\section{Analysis 6.3. Comparison 6 More structured vs less structured reduction, Outcome 3 Pre-quit reduction in cpd.}

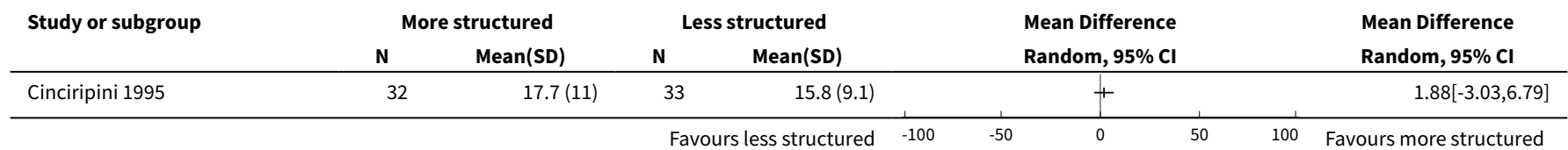

Analysis 6.4. Comparison 6 More structured vs less structured reduction, Outcome 4 Pre-quit tobacco withdrawal.

\begin{tabular}{|c|c|c|c|}
\hline \multicolumn{4}{|c|}{ Pre-quit tobacco withdrawal } \\
\hline Study & Withdrawal measurement method & Withdrawal measure findings & $\begin{array}{l}\text { Additional narrative } \\
\text { information on AEs }\end{array}$ \\
\hline Cinciripini 1995 & $\begin{array}{l}\text { "Hughes (Minnesota) Withdrawal } \\
\text { Symptoms Checklist (Hughes 1986), } \\
\text { which provided a total score using rat- } \\
\text { ings from } 0 \text { to } 3 \text { of } 17 \text { symptoms of to- } \\
\text { bacco withdrawal (e.g., irritability, } \\
\text { hunger, sleep disturbance, etc.)" N.B. } \\
\text { this description of the Minnesota Nico- } \\
\text { tine Withdrawal Scale does not appear } \\
\text { to be accurate - unclear whether inves- }\end{array}$ & $\begin{array}{l}\text { Estimated from Figure } 1 \text { in main paper: } \\
\text { Scheduled reduction group: baseline } \\
10.6 \text {; quit day } 15.8 \text { (change }+5.2 \text { ) } \\
\text { Non-scheduled reduction group: base- } \\
\text { line } 11.2 ; \text { quit day } 19.2 \text { (change }+8.0 \text { ) } \\
\text { Therefore, withdrawal symptoms went } \\
\text { up in both study arms and more in the } \\
\text { scheduled reduction arm (this differ- } \\
\text { ence was not tested for significance) }\end{array}$ & $\mathrm{n} / \mathrm{a}$ \\
\hline
\end{tabular}




\begin{tabular}{|c|c|c|c|}
\hline \multicolumn{4}{|c|}{ Pre-quit tobacco withdrawal } \\
\hline Study & Withdrawal measurement method & Withdrawal measure findings & $\begin{array}{l}\text { Additional narrative } \\
\text { information on AEs }\end{array}$ \\
\hline & $\begin{array}{l}\text { tigators implemented in this way or as } \\
\text { intended by the authors }\end{array}$ & & \\
\hline
\end{tabular}

\section{Comparison 7. Additional behavioural SC components}

\begin{tabular}{|c|c|c|c|c|}
\hline Outcome or subgroup title & $\begin{array}{l}\text { No. of } \\
\text { studies }\end{array}$ & $\begin{array}{l}\text { No. of } \\
\text { partici- } \\
\text { pants }\end{array}$ & Statistical method & Effect size \\
\hline 1 Abstinence & 4 & 1033 & $\begin{array}{l}\text { Risk Ratio (M-H, Random, 95\% } \\
\mathrm{Cl})\end{array}$ & $0.79[0.34,1.84]$ \\
\hline $\begin{array}{l}1.1 \text { Twice weekly vs weekly behavioural } \\
\text { support }\end{array}$ & 1 & 56 & $\begin{array}{l}\text { Risk Ratio (M-H, Random, 95\% } \\
\mathrm{Cl} \text { ) }\end{array}$ & $0.41[0.15,1.13]$ \\
\hline 1.2 NRT adherence counselling & 1 & 928 & $\begin{array}{l}\text { Risk Ratio (M-H, Random, 95\% } \\
\mathrm{Cl} \text { ) }\end{array}$ & $1.73[1.09,2.74]$ \\
\hline 1.3 Peer support & 1 & 24 & $\begin{array}{l}\text { Risk Ratio (M-H, Random, 95\% } \\
\mathrm{Cl})\end{array}$ & $0.67[0.13,3.30]$ \\
\hline 1.4 Motivational self-talk & 1 & 25 & $\begin{array}{l}\text { Risk Ratio (M-H, Random, 95\% } \\
\mathrm{Cl} \text { ) }\end{array}$ & $0.54[0.17,1.70]$ \\
\hline 2 Pre-quit reduction in cpd & 1 & & $\begin{array}{l}\text { Mean Difference (IV, Random, } \\
95 \% \mathrm{CI} \text { ) }\end{array}$ & Totals not selected \\
\hline $\begin{array}{l}2.1 \text { Twice weekly vs weekly behavioural } \\
\text { support }\end{array}$ & 1 & & $\begin{array}{l}\text { Mean Difference (IV, Random, } \\
95 \% \mathrm{CI})\end{array}$ & $0.0[0.0,0.0]$ \\
\hline
\end{tabular}

Analysis 7.1. Comparison 7 Additional behavioural SC components, Outcome 1 Abstinence.

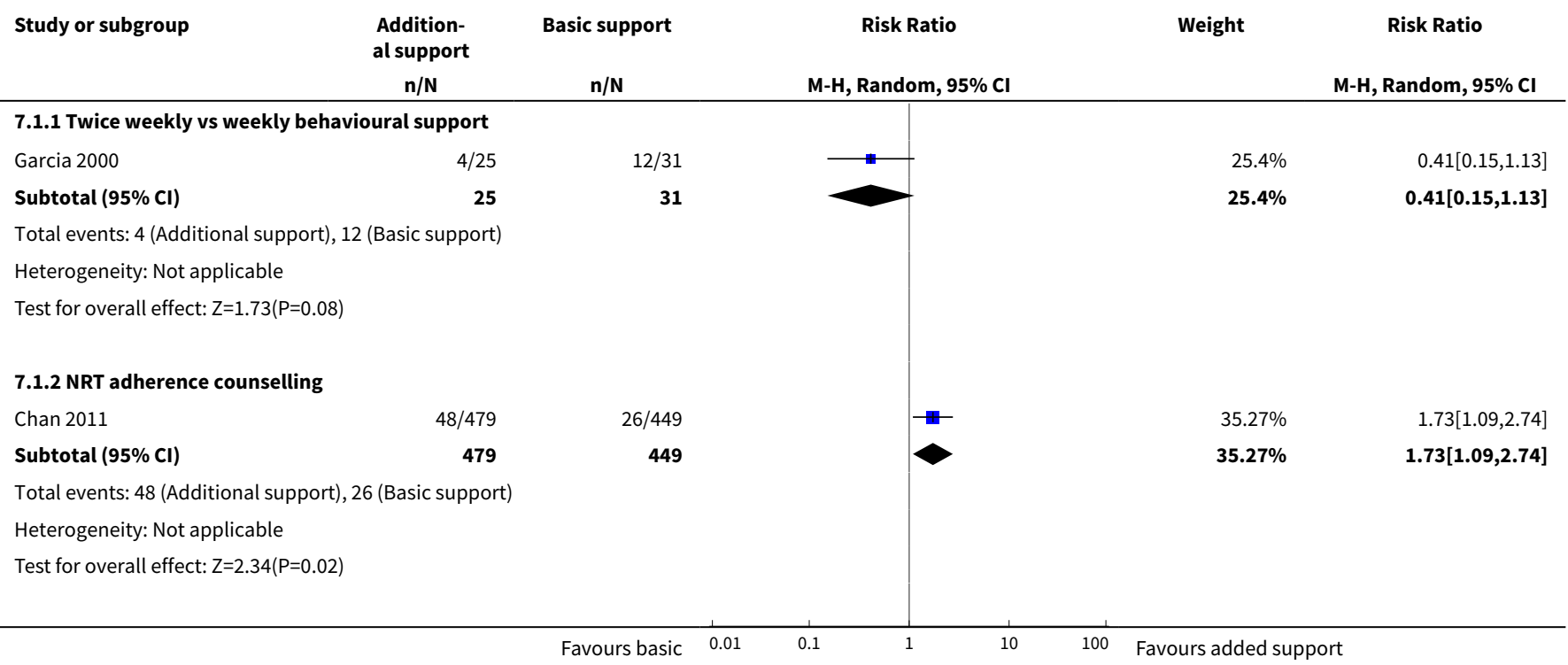




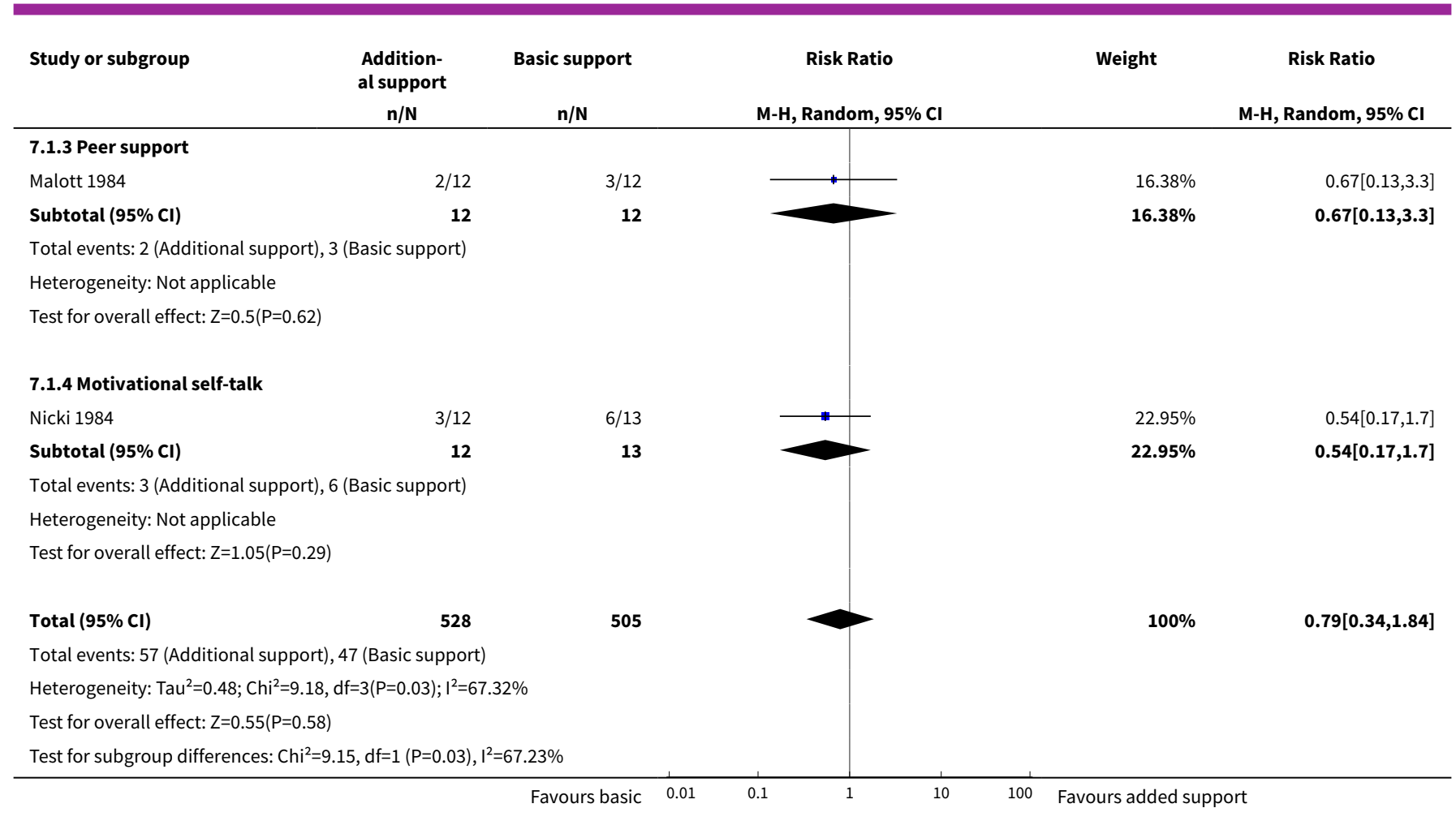

Analysis 7.2. Comparison 7 Additional behavioural SC components, Outcome 2 Pre-quit reduction in cpd.

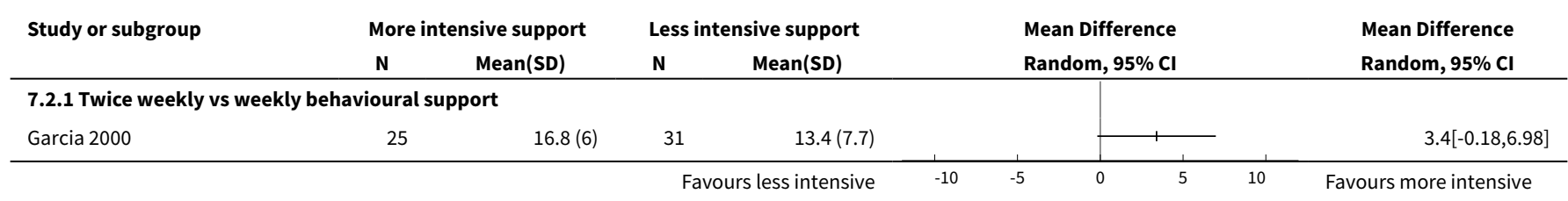

\section{Comparison 8. Behavioural reduction versus nicotine fading}

\begin{tabular}{lllll}
\hline Outcome or subgroup title & $\begin{array}{l}\text { No. of } \\
\text { studies }\end{array}$ & $\begin{array}{l}\text { No. of } \\
\text { partici- } \\
\text { pants }\end{array}$ & Statistical method & Effect size \\
\hline 1 Abstinence & 3 & Risk Ratio $(\mathrm{M}-\mathrm{H}$, Random, 95\% Cl) & Totals not selected \\
\hline 1.1 Reduction vs nicotine fading & 1 & Risk Ratio (M-H, Random, 95\% Cl) & $0.0[0.0,0.0]$ \\
\hline $\begin{array}{l}1.2 \text { Reduction + nicotine fading vs } \\
\text { nicotine fading only }\end{array}$ & 1 & Risk Ratio (M-H, Random, $95 \% \mathrm{Cl})$ & $0.0[0.0,0.0]$ \\
\hline $\begin{array}{l}1.3 \text { Reduction alone vs reduction }+ \\
\text { nicotine fading }\end{array}$ & 1 & Risk Ratio (M-H, Random, 95\% Cl) & $0.0[0.0,0.0]$ \\
\hline \begin{tabular}{l}
2 Pre-quit reduction in cotinine \\
\hline
\end{tabular} & 1 & Mean Difference (IV, Random, $95 \% \mathrm{Cl})$ & Totals not selected \\
\hline
\end{tabular}




\begin{tabular}{lllll}
\hline Outcome or subgroup title & $\begin{array}{l}\text { No. of } \\
\text { studies }\end{array}$ & $\begin{array}{l}\text { No. of } \\
\text { partici- } \\
\text { pants }\end{array}$ & Statistical method & Effect size \\
\hline $\begin{array}{l}2.1 \text { Reduction alone vs nicotine } \\
\text { fading + reduction }\end{array}$ & 1 & Mean Difference (IV, Random, 95\% Cl) & $0.0[0.0,0.0]$ \\
\hline 3 Pre-quit tobacco withdrawal & & Other data & No numeric data \\
\hline
\end{tabular}

Analysis 8.1. Comparison 8 Behavioural reduction versus nicotine fading, Outcome 1 Abstinence.

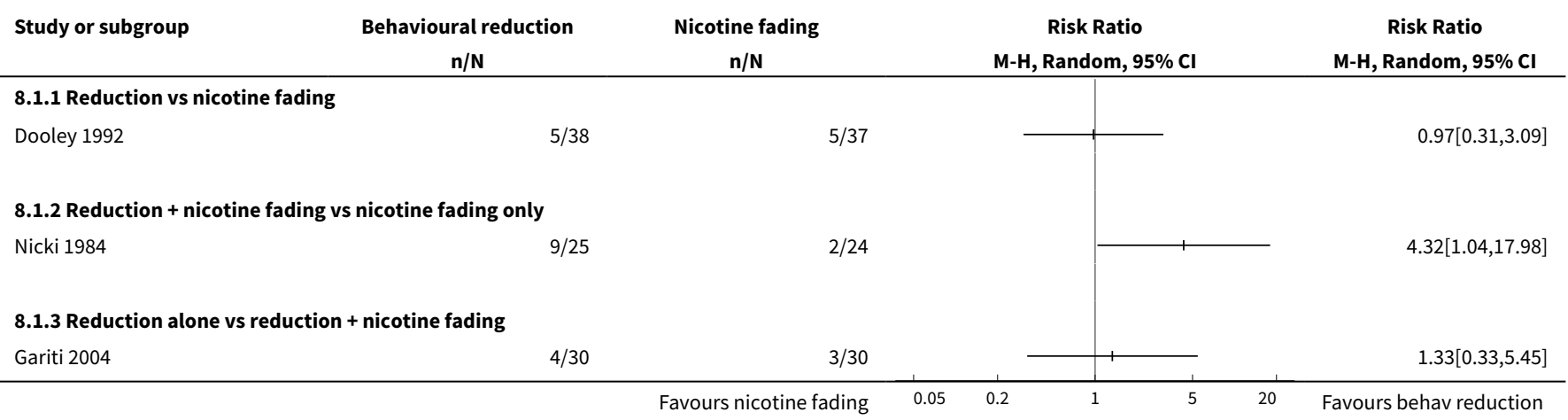

Analysis 8.2. Comparison 8 Behavioural reduction versus nicotine fading, Outcome 2 Pre-quit reduction in cotinine.

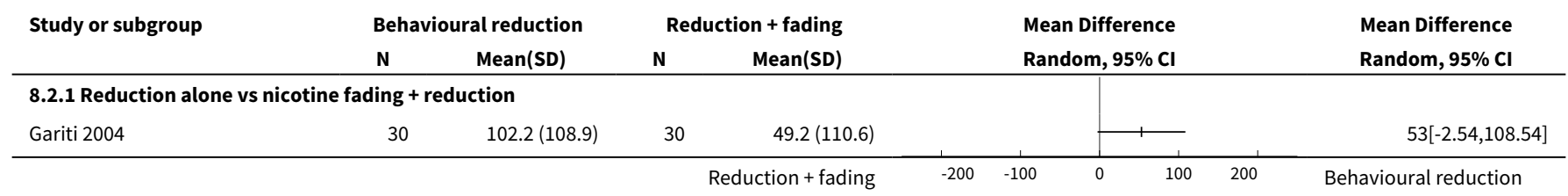

Analysis 8.3. Comparison 8 Behavioural reduction versus nicotine fading, Outcome 3 Pre-quit tobacco withdrawal.

\begin{tabular}{|c|c|c|}
\hline \multicolumn{3}{|c|}{ Pre-quit tobacco withdrawal } \\
\hline Study & Heading 1 & Heading 2 \\
\hline Gariti 2004 & $\begin{array}{l}\text { Minnesota Nicotine Withdrawal Scale (Hughes 1986), } \\
\text { measured using scales of } 0 \text { to } 4 \text { across } 8 \text { symptoms } \\
\text { (craving for cigarettes; restlessness; increased ap- } \\
\text { petite; depressed mood; anxiety; difficulty concentrat- } \\
\text { ing; irritability, frustration or anger; and sleep prob- } \\
\text { lems), and completed daily }\end{array}$ & $\begin{array}{l}\text { "A mixed-effects model analysis did not reveal treat- } \\
\text { ment group by time }(F(7,239)=0.6, p=.76) \text { or treat- } \\
\text { ment group differences }(F(1,46)=0.02, p=.88) \text { for } \\
\text { weekly changes in withdrawal scores from baseline } \\
\text { through 1-week post-treatment completion" "With- } \\
\text { drawal scores were consistently low indicative of } \\
\text { slight withdrawal" }\end{array}$ \\
\hline
\end{tabular}




\section{Comparison 9. Other comparisons}

\begin{tabular}{|c|c|c|c|c|}
\hline Outcome or subgroup title & $\begin{array}{l}\text { No. of } \\
\text { studies }\end{array}$ & $\begin{array}{l}\text { No. of } \\
\text { partici- } \\
\text { pants }\end{array}$ & Statistical method & Effect size \\
\hline 1 Abstinence & 4 & & Risk Ratio (M-H, Random, 95\% Cl) & $\begin{array}{l}\text { Totals not select- } \\
\text { ed }\end{array}$ \\
\hline 1.1 Aerobic exercise vs health education & 1 & & Risk Ratio (M-H, Random, 95\% Cl) & $0.0[0.0,0.0]$ \\
\hline 1.2 Gradual reduction vs partially gradual & 1 & & Risk Ratio (M-H, Random, 95\% Cl) & $0.0[0.0,0.0]$ \\
\hline $\begin{array}{l}1.3 \text { Contingent vs non-contingent vouch- } \\
\text { ers }\end{array}$ & 1 & & Risk Ratio (M-H, Random, 95\% Cl) & $0.0[0.0,0.0]$ \\
\hline $\begin{array}{l}1.4 \text { Greater targets }+ \text { choice of abstinence } \\
\text { vs minimal reduction + clear abstinence } \\
\text { target }\end{array}$ & 1 & & Risk Ratio (M-H, Random, 95\% Cl) & $0.0[0.0,0.0]$ \\
\hline 2 Pre-quit reduction in cpd & 1 & & Mean Difference (IV, Random, 95\% Cl) & $\begin{array}{l}\text { Totals not select- } \\
\text { ed }\end{array}$ \\
\hline $\begin{array}{l}2.1 \text { Greater targets + choice of abstinence } \\
\text { vs minimal reduction + clear abstinence } \\
\text { target }\end{array}$ & 1 & & Mean Difference (IV, Random, 95\% Cl) & $0.0[0.0,0.0]$ \\
\hline 3 Pre-quit reduction in $\mathrm{CO}$ & 1 & & Mean Difference (IV, Random, 95\% Cl) & Subtotals only \\
\hline $\begin{array}{l}\text { 3.1 Greater targets + choice of abstinence } \\
\text { vs minimal reduction + clear abstinence } \\
\text { target }\end{array}$ & 1 & 66 & Mean Difference (IV, Random, 95\% Cl) & $3.40[-4.59,11.39]$ \\
\hline $\begin{array}{l}4 \text { Pre-quit tobacco withdrawal \& addi- } \\
\text { tional AE information }\end{array}$ & & & Other data & No numeric data \\
\hline
\end{tabular}

Analysis 9.1. Comparison 9 Other comparisons, Outcome 1 Abstinence.

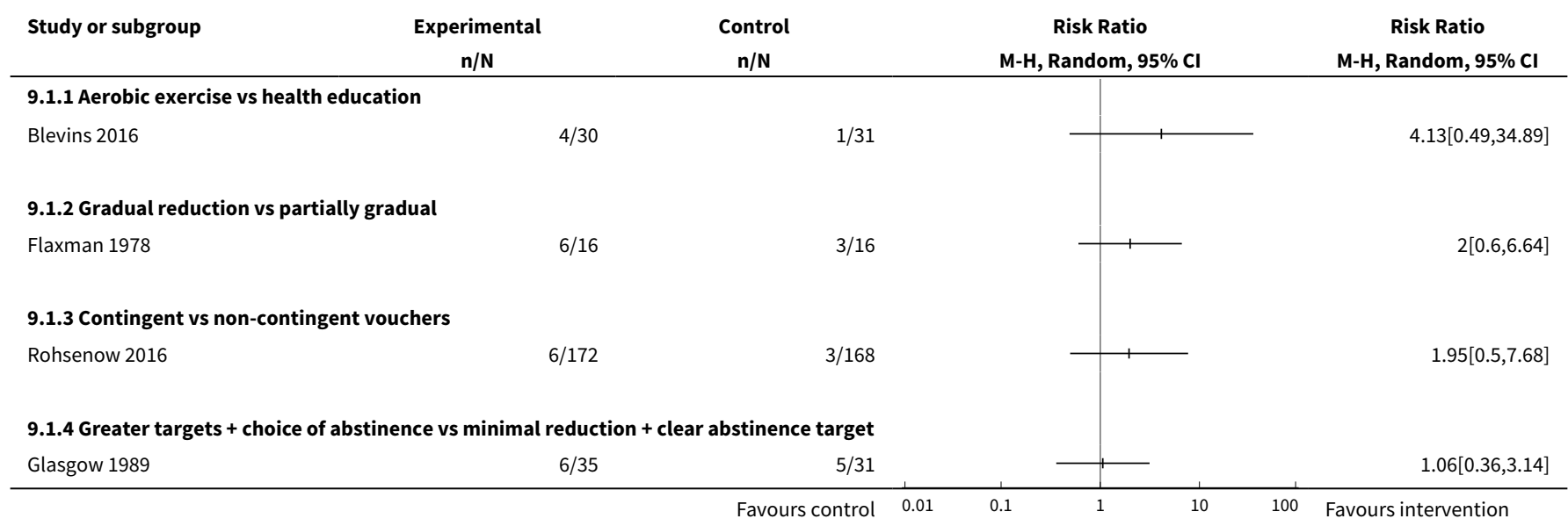


Analysis 9.2. Comparison 9 Other comparisons, Outcome 2 Pre-quit reduction in cpd.

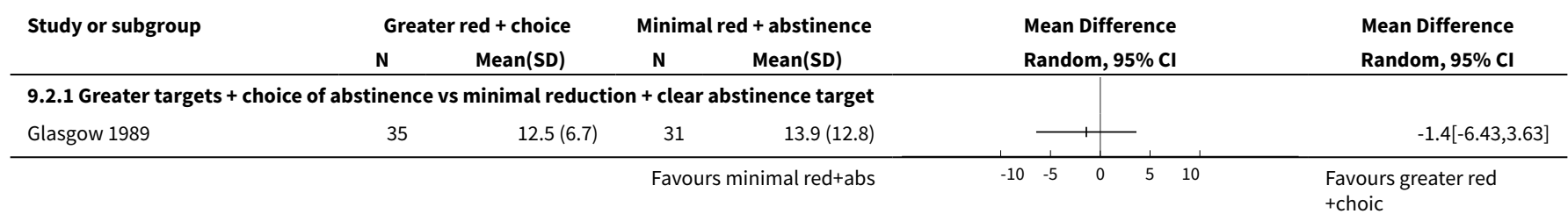

Analysis 9.3. Comparison 9 Other comparisons, Outcome 3 Pre-quit reduction in co.

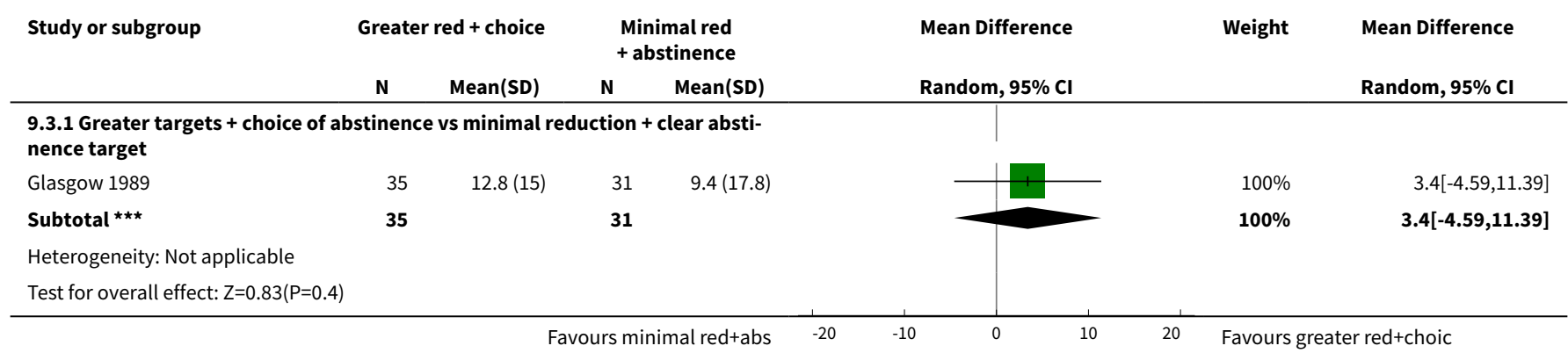

Analysis 9.4. Comparison 9 Other comparisons, Outcome 4 Pre-quit tobacco withdrawal \& additional AE information.

\begin{tabular}{|c|c|c|c|}
\hline \multicolumn{4}{|c|}{ Pre-quit tobacco withdrawal \& additional AE information } \\
\hline Study & Withdrawal measurement method & Withdrawal measure findings & $\begin{array}{l}\text { Additional narrative } \\
\text { information on AEs }\end{array}$ \\
\hline Glasgow 1989 & $\begin{array}{l}\text { Checklist modified from the Co- } \\
\text { hen-Hobermann Inventory of Physical } \\
\text { Symptoms (Cohen 1983) at baseline } \\
\text { and session immediately following quit } \\
\text { attempts }\end{array}$ & $\begin{array}{l}\text { There were no significant be- } \\
\text { tween-group differences }\end{array}$ & $\mathrm{n} / \mathrm{a}$ \\
\hline
\end{tabular}

\section{AP P E N D I C E S}

\section{Appendix 1. Register Search}

\#1 "cold turkey"

\#2 schedul* NEAR3 smok ${ }^{*}$

\#3 "cut down" or cut-down

\#4 Gradual * NEAR3 (reduc* or quit* or stop* or abstin * or abstain* or cessat*

\#5 Abrupt* NEAR3 (reduc* or quit* or stop* or abstin* or abstain* or cessat ${ }^{\star}$ )

\#6 fading

\#7 taper*

\#8 controlled NEXT smoking

\#9 Smoking reduction/

\#10 smoking reduction.mp

\#11 \#1 OR \#2 OR \#3 OR \#4 OR \#5 OR \#6 OR \#7 OR \#8 OR \#9 OR 10\#

\section{Appendix 2. Original MEDLINE search strategy}

1 cold turkey.mp. 
$2\left(\right.$ schedul $^{\star}$ adj3 smok $\left.{ }^{\star}\right) \cdot m p$.

3 (cut $^{\star}$ down or cut-down).mp.

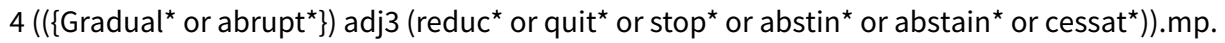

5 fading.mp.

6 taper $^{\star} . \mathrm{mp}$.

7 controlled smoking.mp.

8 Smoking reduction/ or smoking reduction.mp.

91 or 2 or 3 or 4 or 5 or 6 or 7 or 8

10 Randomized Controlled Trial.pt.

11 Controlled Clinical Trial.pt.

12 Pragmatic Clinical Trial.pt.

13 Equivalence Trial.pt.

14 Adaptive Clinical Trial.pt.

15 Clinical Trial.pt.

16 Meta analysis.pt.

17 exp Clinical Trial/

18 Random-Allocation/

19 randomized-controlled trials/

20 double-blind-method/

21 single-blind-method/

22 placebos/

23 Research-Design/

24 ((clin\$ adj5 trial\$) or placebo\$ or random\$).ti,ab.

25 ((singl\$ or doubl\$ or trebl\$ or tripl\$) adj5 (blind\$ or mask\$)).ti,ab.

26 (volunteer\$ or prospectiv\$).ti,ab.

27 exp Follow-Up-Studies/

28 exp Retrospective-Studies/

29 exp Prospective-Studies/

30 exp Evaluation-Studies/ or Program-Evaluation.mp. [mp=title, abstract, original title, name of substance word, subject heading word, floating sub-heading word, keyword heading word, protocol supplementary concept word, rare disease supplementary concept word, unique identifier, synonyms]

31 exp Cross-Sectional-Studies/

32 Comparative study/

33 exp Behavior-therapy/

34 exp Health-Promotion/

35 exp Community-Health-Services/

Smoking reduction interventions for smoking cessation (Review) 
36 exp Health-Behavior/ or exp Health-Education/

3710 or 11 or 12 or 13 or 14 or 15 or 16 or 17 or 18 or 19 or 20 or 21 or 22 or 23 or 24 or 25 or 26 or 27 or 28 or 29 or 30 or 31 or 32 or 33 or 34 or 35 or 36

38 smoking cessation.mp. or exp Smoking Cessation/

39 tobacco cessation.mp. or "Tobacco-Use-Cessation"/

40 exp Smoking/th [Therapy]

41 "Tobacco-Use-Disorder"/

42 Tobacco-Smokeless/

43 exp Tobacco-Smoke-Pollution/

44 Smoking reduction/ or Smoking reduction.mp.

45 Smoking prevention/

46 Vaping/ or vaping.mp.

47 Electronic Nicotine Delivery Systems/

48 electronic cigar*.mp.

49 exp Pipe smoking/ or exp Tobacco smoking/ or exp Tobacco Products/

50 ((quit\$ or stop\$ or ceas\$ or giv\$ or abstain* or abstinen*) adj5 (smoking or smoke* or tobacco)).ti,ab.

51 exp Tobacco/ or exp Nicotine/

5238 or 39 or 40 or 41 or 42 or 43 or 44 or 45 or 46 or 47 or 48 or 49 or 50 or 51 [A category smoking terms]

53 exp Smoking/ not 52 [B category smoking terms]

5410 or 11 or 12 or 13 or 14 [Likely Controlled trial design terms; RCTs, CCTs, Pragmatic trials, Equivalence trials, Adaptive clinical trials. ] 5552 and 37 [A category smoking+all design terms]

5652 and 54 [A category smoking terms+likely CT design terms]

57 (animals not humans).sh. [used with 'not' to exclude animal studies for each subset]

58 ((38 or 39 or 41 or 42) and REVIEW.pt.) not 55 [Set 4: Core smoking related reviews only]

5953 and 37 [B category smoking+all design terms]

60 (59 and 54) not 57 [Set 3: B smoking terms, likely CT design terms, human only]

6155 not 56 not 57 [Set 2: A smoking terms, not core CT terms, human only]

62 (52 and 54) not 57 [Set 1: A smoking terms, likely CT design terms, human only]

639 and 62

649 and 61

659 and 60

6663 or 64 or 65

\section{Appendix 3. Original Embase search strategy}

1 cold turkey.mp.

$2\left(\right.$ schedul $^{\star}$ adj3 smok $\left.{ }^{\star}\right) \cdot m p$. 
3 (cut $^{\star}$ down or cut-down).mp.

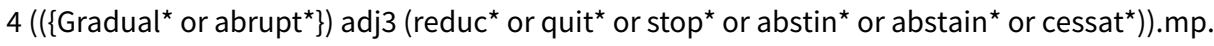

5 fading.mp.

6 taper $^{\star} . \mathrm{mp}$.

7 controlled smoking.mp.

8 smoking reduction/ or smoking reduction.mp.

91 or 2 or 3 or 4 or 5 or 6 or 7 or 8

10 random\$.ti,ab.

11 factorial\$̣.ti,ab.

12 (cross over\$ or crossover\$ or cross-over\$).ti,ab.

13 placebo\$.ti,ab.

14 (double\$ adj blind\$).ti,ab.

15 (single\$ adj blind\$).ti,ab.

16 assign $\$ . t i, a b$.

17 allocat\$.ti,ab.

18 volunteer\$.ti,ab.

19 CROSSOVER PROCEDURE.sh.

20 DOUBLE-BLIND PROCEDURE.sh.

21 RANDOMIZED CONTROLLED TRIAL.sh.

22 SINGLE-BLIND PROCEDURE.sh.

23 or $/ 10-22$

24 smoking cessation.mp.

25 exp smoking cessation/ or smoking cessation program/

26 exp smoking-/ [Not used as single term after Dec 2010]

2726 and (( (quit\$ or stop\$ or ceas\$ or giv\$ or prevent\$) adj3 smok\$) or cigarette\$).ti,ab.

28 exp passive smoking/

29 exp smoking habit/

30 smokeless tobacco/

31 smoking reduction/

3224 or 25 or 27 or 28 or 29 or 30 or 31

3323 and 32

349 and 33

Appendix 4. Original PsycINFO search strategy

1 cold turkey.mp.

$2\left(\right.$ schedul $^{\star}$ adj3 smok $\left.{ }^{\star}\right) \cdot m p$. 


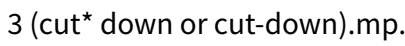

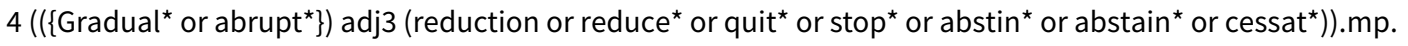

5 fading.mp.

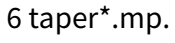

7 controlled smoking.mp.

8 smoking reduction.mp.

91 or 2 or 3 or 4 or 5 or 6 or 7 or 8

10 smoking cessation.mp. or exp Smoking Cessation/

11 (antismoking or anti-smoking).mp. [mp=title, abstract, heading word, table of contents, key concepts, original title, tests \& measures]

12 (quit\$ or cessat\$).mp.

13 (abstin\$ or abstain\$).mp.

14 (control adj smok\$).mp.

15 exp behavior modification/

1611 or 12 or 13 or 14 or 15

17 tobacco-smoking/

18 (smok\$ or cigar\$ or tobacco\$).mp.

19 Prevention/

2017 or 18

2116 and 20

2219 and 20

2310 or 21 or 22

249 and 23

\section{CONTRIBUTIONS OF AUTHORS}

All authors were involved in study screening and eligibility assessment, data extraction and 'Risk of bias' assessment. JMOM provided statistical expertise. NL wrote the first draft of the report and all authors commented on that draft.

\section{DECLARATIONS OF INTEREST}

BH has no known conflicts of interest.

EK has no known conflicts of interest. EK was an author of one included study (Klemperer 2017). They did not carry out data extraction or risk of bias assessment for this study.

JMOM has no known conflicts of interest.

NL has no known conflicts of interest. NL was an author of one included study (Lindson-Hawley 2016b). They did not carry out data extraction or risk of bias assessment for this study.

PA has no known conflicts of interest. PA was an author of two included studies (Farley 2017; Lindson-Hawley 2016b) They did not carry out data extraction or risk of bias assessment for those studies. 


\section{SOURCES OF SUPPORT}

\section{Internal sources}

- National Institute for Health Research (NIHR), UK.

The infrastructure of the Cochrane Tobacco Addiction Group is funded by the NIHR, and review was carried out as part of a Cochrane Programme Grant funded by the NIHR. NL is a Managing Editor for the group, PA is the group's Co-ordinating Editor, and JMOM provides statistical support for the group's programme grant.

\section{External sources}

- University of Oxford, UK. JMOM, NL \& PA's salaries were paid by the University of Oxford

- University of Vermont, USA.

EK's salary was paid by the University of Vermont

\section{DIFFERENCES BETWEEN PROTOCOLANDREVIEW}

- We specified in our protocol that we would include quasi-RCTs, as we were expecting a paucity of eligible studies. However, our searches resulted in more evidence than we were expecting and so we made the decision to exclude non-randomised studies to maximise the validity of the review.

- We originally stated that we would assess 'selective outcome reporting' as part of our 'Risk of bias' assessment, but we ultimately followed the 'Risk of bias' guidance developed by the Cochrane Tobacco Addiction Group. This guidance advises authors not to assess this domain, due to a difficulty in doing so in studies predating trial registration (many of these were included in this review).

- We specified in the protocol that we would not assess performance bias in line with the Cochrane Tobacco Addiction Group's guidance for assessing risks of bias in trials of behavioural interventions. However, we identified a subset of studies that compared a reduction-toquit intervention alongside pharmacotherapy versus the same reduction intervention alongside a placebo or no pharmacotherapy. We therefore deemed it appropriate to assess performance bias in these studies only.

- We originally proposed a sensitivity analysis comparing outcomes on an intention-to-treat basis versus complete-case analysis. We have since decided to carry out a wider investigation of this issue incorporating a wider range of reviews, and so will do this outside of this review.

- For pragmatic reasons, we were unable to report on cigarettes per day (cpd) as a moderator of the effect of reduction-to-quit interventions. 